\title{
Soot Emission Factors from Lab-Scale Flares Burning Solution Gas Mixtures
}

By

James D.N. McEwen

A thesis submitted to

The Faculty of Graduate Studies and Research

in partial fulfilment of

the degree requirements of

Master of Applied Science in Mechanical Engineering

Ottawa-Carleton Institute for

Mechanical and Aerospace Engineering

Department of Mechanical and Aerospace Engineering

Carleton University

Ottawa, Ontario, Canada

April 2010

Copyright $(\mathbb{C}$

2010 - James D.N. McEwen 


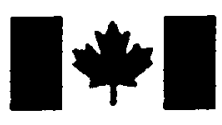

\section{Library and Archives} Canada

Published Heritage Branch

395 Wellington Street Ottawa ON K1A ON4 Canada
Bibliotheque et

Archives Canada

Direction du

Patrimoine de l'édition

395 , rue Wellington Ottawa ON K1A ON4

Canada
Your file Votre référence

ISBN: 978-0-494-68639-3

Our file Notre référence

ISBN: 978-0-494-68639-3
NOTICE:

The author has granted a nonexclusive license allowing Library and Archives Canada to reproduce, publish, archive, preserve, conserve, communicate to the public by telecommunication or on the Internet, loan, distribute and sell theses worldwide, for commercial or noncommercial purposes, in microform, paper, electronic and/or any other formats.

The author retains copyright ownership and moral rights in this thesis. Neither the thesis nor substantial extracts from it may be printed or otherwise reproduced without the author's permission.

\begin{abstract}
AVIS:
L'auteur a accordé une licence non exclusive permettant à la Bibliothèque et Archives Canada de reproduire, publier, archiver, sauvegarder, conserver, transmettre au public par télécommunication ou par l'Internet, prêter, distribuer et vendre des thèses partout dans le monde, à des fins commerciales ou autres, sur support microforme, papier, électronique et/ou autres formats.
\end{abstract}

L'auteur conserve la propriété du droit d'auteur et des droits moraux qui protège cette thèse. Ni la thèse ni des extraits substantiels de celle-ci ne doivent être imprimés ou autrement reproduits sans son autorisation.
In compliance with the Canadian Privacy Act some supporting forms may have been removed from this thesis.

While these forms may be included in the document page count, their removal does not represent any loss of content from the thesis.
Conformément à la loi canadienne sur la protection de la vie privée, quelques formulaires secondaires ont été enlevés de cette thèse.

Bien que ces formulaires aient inclus dans la pagination, il n'y aura aucun contenu manquant. 
The undersigned recommend to the Faculty of Graduate Studies and Research acceptance of the thesis

\title{
Soot Emission Factors from Lab-Scale Flares Burning Solution Gas Mixtures
}

\author{
Submitted by James D.N. McEwen \\ in partial fulfilment of the requirements for the degree of \\ Master of Applied Science in Mechanical Engineering
}

Dr. Matthew Johnson, Thesis Supervisor

Dr. Kevin Thomson, Thesis Co-supervisor

Dr. Metin Yaras, Chair, Department of Mechanical and Aerospace Engineering

Carleton University

2010 


\section{Abstract}

A repeatable soot sampling protocol was developed to measure soot emission factors $(E F)$ from lab-scale flares burning fuel compositions representative of solution gas flares typical of the Alberta upstream oil and gas industry. A gravimetric analysis system was employed as well as a Laser Induced Incandescence (LII) system. Results showed that accurate soot $E F$ s can be quantified and that results from the two measurement techniques were in good agreement. For the range of conditions tested (burner inner diameter of 12.7-76.2 mm, exit velocity $0.1-2.2 \mathrm{~m} / \mathrm{s}$, and several 4- and 6-component fuel mixtures consisting of 74.5 to $91.1 \%$ methane), measured soot emission rates were less than $1 \mathrm{~g} \mathrm{soot} / \mathrm{kg}$ fuel (or $0.84 \mathrm{~kg} \mathrm{soot} / 10^{3} \mathrm{~m}^{3}$ fuel). These values are lower than the current $E F$ used in Canada $\left(2.5632 \mathrm{~kg}\right.$ soot $/ 10^{3} \mathrm{~m}^{3}$ fuel). A simple empirical relationship was established for the emission factor as a function of the fuel heating value for a range of conditions, which is a significant improvement over the single $E F$ approach currently adopted. 


\section{Acknowledgements}

This thesis would not have been possible without the unwavering support of my supervisor, Professor Johnson. Your enthusiasm and excitement for research is contagious, and your encouragement has kept me going through the harder times. I thank you for all the opportunities you have provided over the years.

I would like to thank all those at the NRC who helped me during my stay in some capacity. Thanks to Greg Smallwood for the use of facilities and resources. To the M-9 technical staff (Faz, both Dans, Bob, and Roland), thanks for the help setting up equipment, troubleshooting the LII, and generally taking time to give advice. Finally, a special thanks to my NRC supervisor Kevin Thomson, for advising me during my time there, and helping me navigate my way through the NRC.

Thanks to my fellow students, particularly Graham, Adam, Steve, Patrizio, and Brian, who have been good friends over the years. Your ability to keep the mood light on darker days and lend a hand or an idea, were always greatly appreciated.

Lastly, I could not have done this without the steadfast support of my family. To Val, I could not have asked for a better partner. I thank you for supporting me in this and all my endeavours. You are truly a special person. 


\section{Table of Contents}

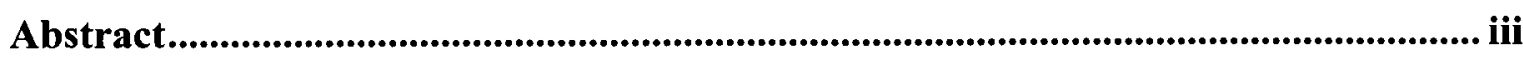

Acknowledgements ................................................................................................ iv

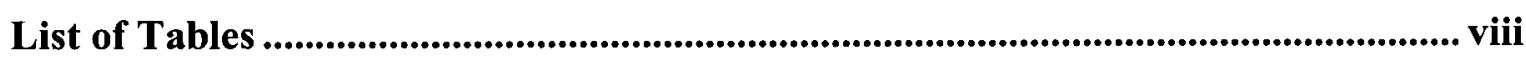

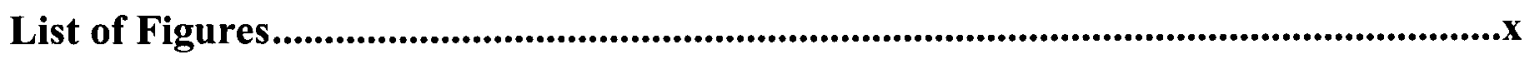

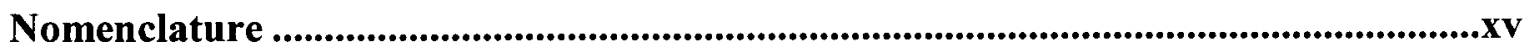

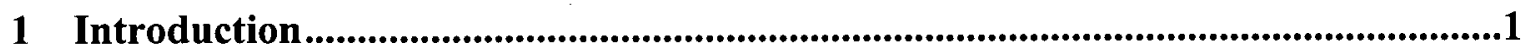

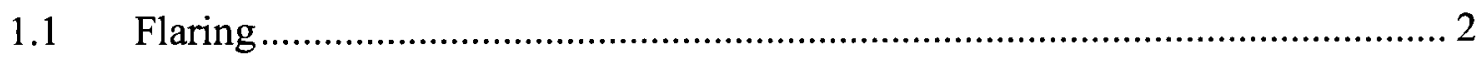

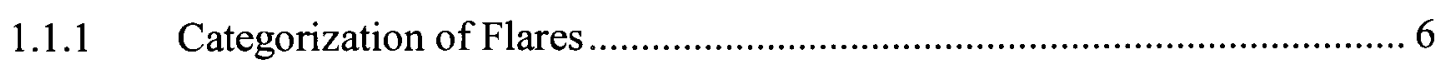

Solution Gas Flares ........................................................................ 6

1.1.3 Flare Efficiency and Soot............................................................ 7

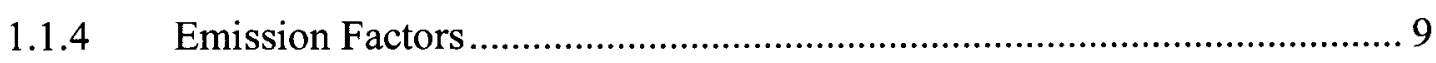

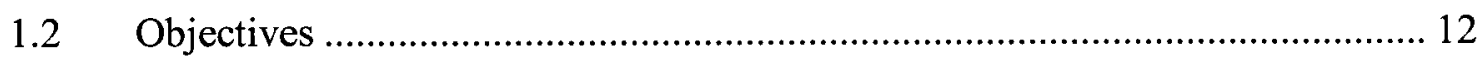

2 A Brief Overview of Soot Formation Theory ................................................14

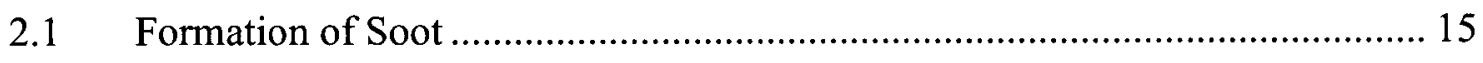

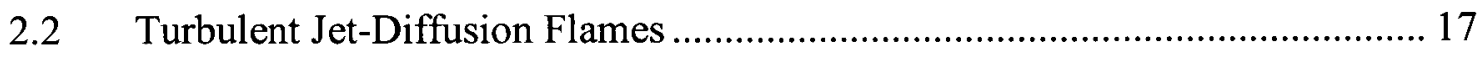

2.2.1 Turbulent Regimes and Flares ..................................................... 20

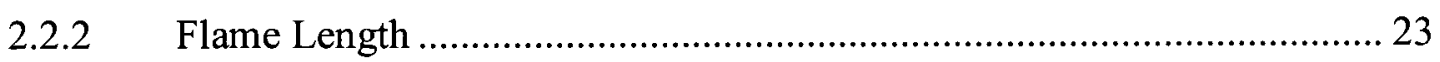

2.3 Soot Emissions from Turbulent Diffusion Flames and Flares ........................ 26

2.3.1 Turbulent, Vertical, Free Jet-Diffusion Flames .................................. 27

2.3.2 Turbulent Diffusion Flames in Cross-Flow Conditions........................... 31

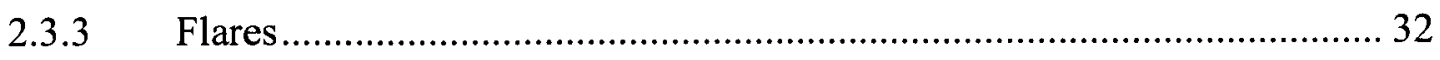

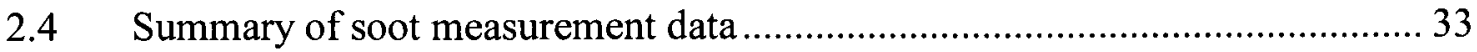

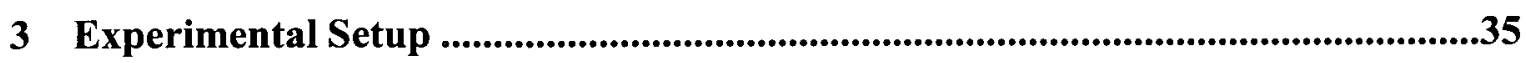

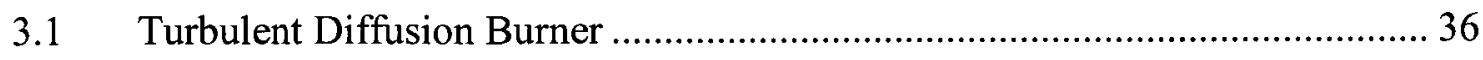

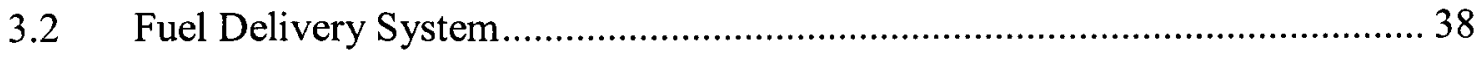

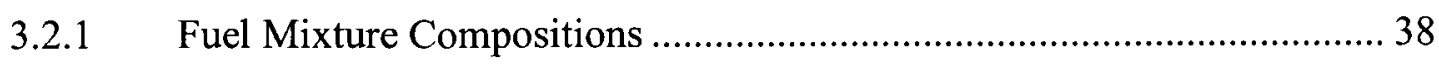


3.2.2 Enclosure and Emission Collection System ........................................... 42

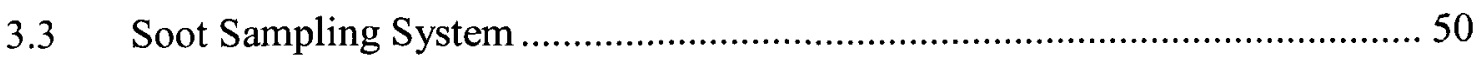

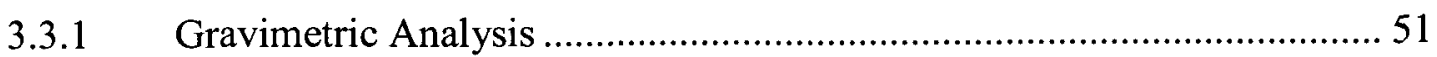

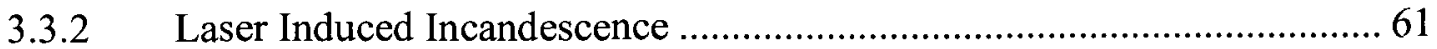

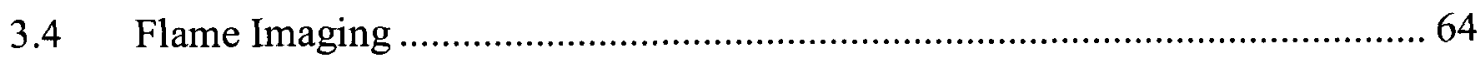

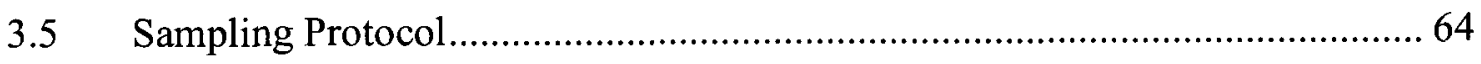

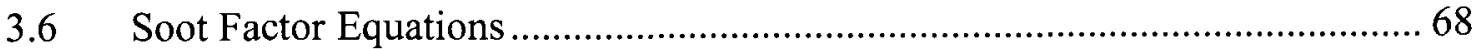

$3.7 \quad$ Uncertainty Analysis................................................................................. 71

3.7.1 Systematic Gravimetric Uncertainty ..................................................... 73

3.7.2 Total Uncertainty in $Y_{s}$ Measured Gravimetrically ................................... 77

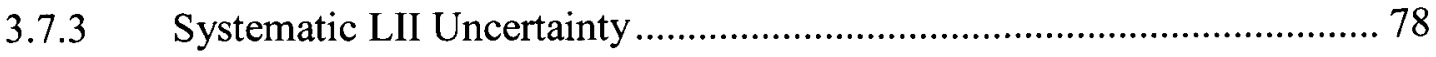

3.7.4 Total Uncertainty in $Y_{s}$ Measured Using LII ......................................... 80

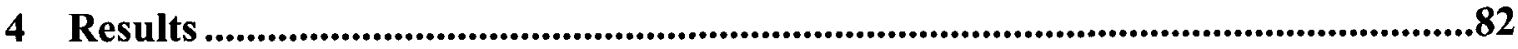

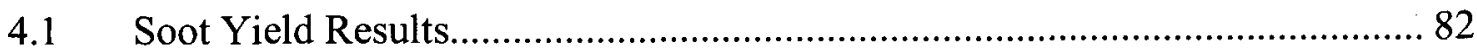

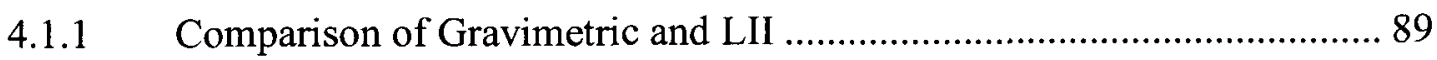

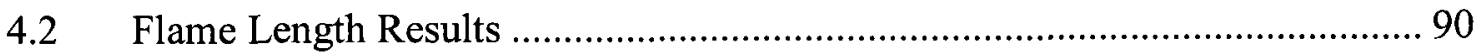

5 Analysis and Discussion.......................................................................................93

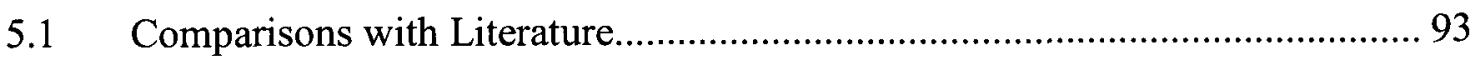

$5.2 \quad$ Potential Effects of Fuel Pre-heating ………….......................................... 98

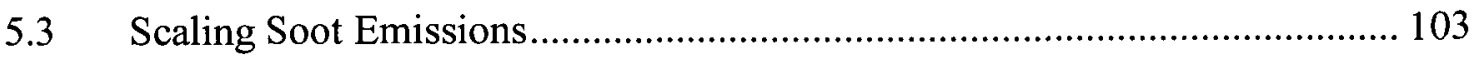

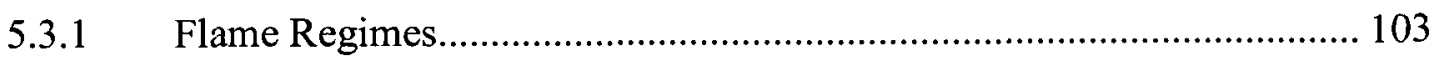

5.3.2 Aerodynamic Scaling Parameters ……………....................................... 107

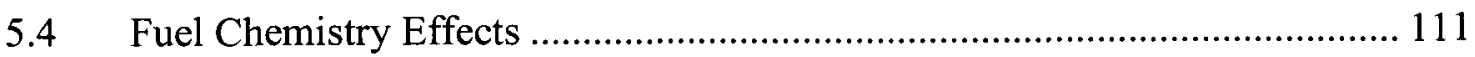

5.4.1 Chemistry-Related Scaling Parameters................................................. 115

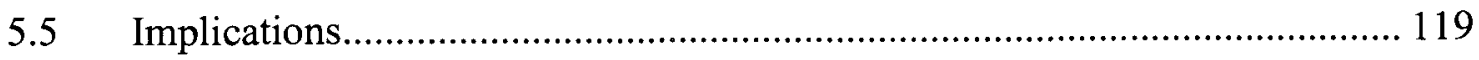

6 Conclusions and Recommendations.............................................................................122

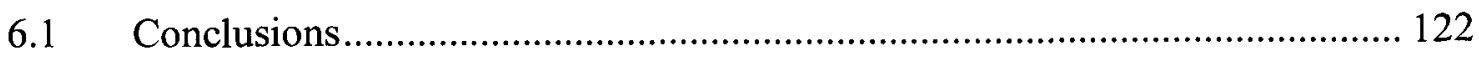

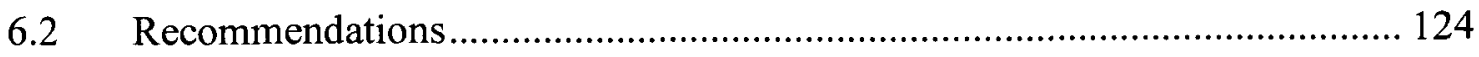

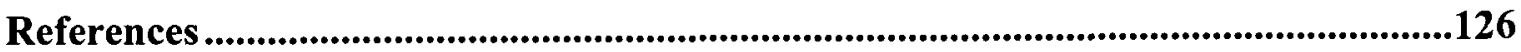

Appendix A .............................................................................................................................133 
Conversion of Soot Emission Factors in AP-42 to NPRI units 133

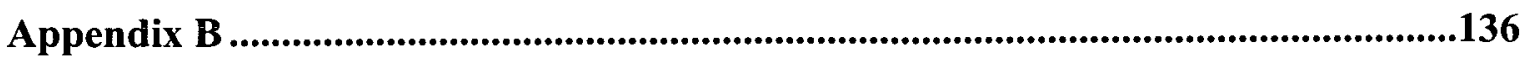

Mass Flow Controller Calibrations and Methods .............................................. 136

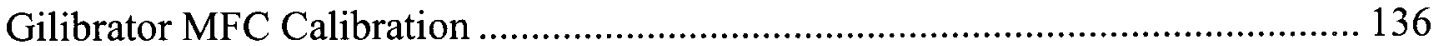

Mass-displacement MFC Calibration ........................................................ 138

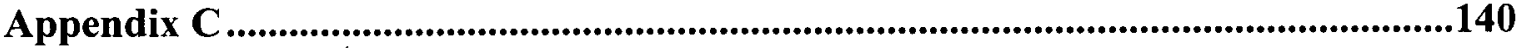

Measurement of Fuel Gas Temperature.......................................................... 140

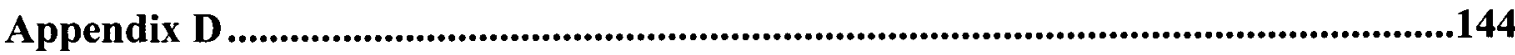

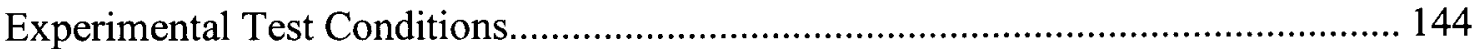




\section{List of Tables}

Table 1.1 - Comparison of Current Emission Factors ................................................... 12

Table 2.1 - Comparison of relevant sooting tendency measurements .............................. 27

Table 2.2 - Summary of relevant soot data ........................................................................ 33

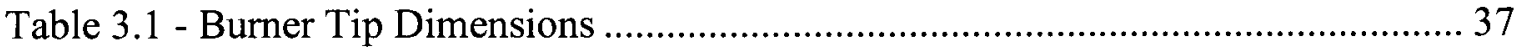

Table 3.2 - Average well composition from 2908 sites in Alberta. C1-C7 represents the number of carbon atoms in the hydrocarbon compound......................................... 39

Table 3.3 - Comparison of sooting tendencies of $n$ - and iso-butane .............................. 41

Table 3.4 - Average, Heavy, and Light fuel mixtures ................................................... 42

Table 3.5 - Comparison of sampling protocols............................................................. 45

Table 3.6 - Comparison of Filter requirements............................................................ 56

Table 3.7 - Comparison of conditioning requirements ....................................................... 59

Table 3.8 - Mean, standard deviation, and standard deviation of the means of control

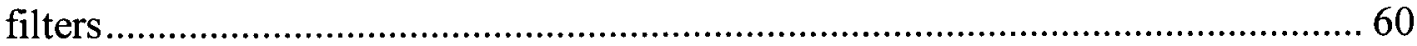

Table 3.9 - Typical contributions to gravimetric systematic uncertainty ..........................78

Table 3.10 - Summary of soot density data from literature ............................................ 79

Table 3.11 - Typical contributions to LII systematic uncertainty ..................................... 81

Table 4.1 - Summary of fuel mixtures used at each test condition..................................... 83

Table A.1 - Estimated, corrected Emissions Factors based on source data from McDaniel

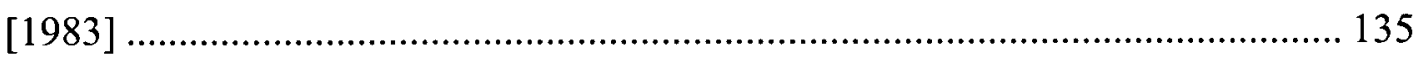

Table B.1 - Calibration values for MFCs.................................................................. 139

Table D. 1 - Average 6-component fuel mixture test conditions $(M W=19.19 \mathrm{~kg} / \mathrm{kmol}$;

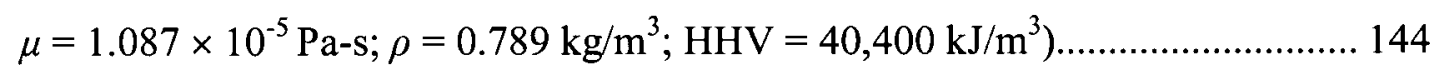

Table D.2 - Light 6-component fuel mixture test conditions $(M W=17.94 \mathrm{~kg} / \mathrm{kmol}$;

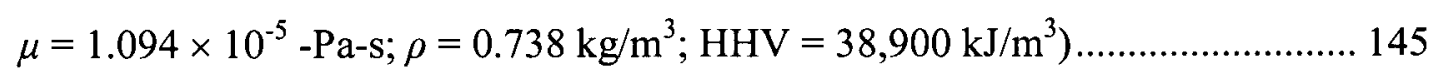

Table D. 3 - Heavy 6-component fuel mixture test conditions $(M W=21.48 \mathrm{~kg} / \mathrm{kmol}$;

$$
\left.\mu=1.072 \times 10^{-5} \mathrm{~Pa}-\mathrm{s} ; \rho=0.883 \mathrm{~kg} / \mathrm{m}^{3} ; \mathrm{HHV}=42,900 \mathrm{~kJ} / \mathrm{m}^{3}\right) \ldots \ldots \ldots \ldots \ldots \ldots \ldots \ldots . .145
$$

Table D. 4 - Average 4-component fuel mixture test conditions $(M W=18.60 \mathrm{~kg} / \mathrm{kmol}$;

$$
\left.\mu=1.065 \times 10^{-5} \mathrm{~Pa}-\mathrm{s} ; \rho=0.765 \mathrm{~kg} / \mathrm{m}^{3} ; \mathrm{HHV}=41,400 \mathrm{~kJ} / \mathrm{m}^{3}\right) \ldots \ldots \ldots \ldots \ldots \ldots \ldots \ldots . .145
$$


Table D.5 - Light 4-component fuel mixture test conditions $(M W=17.93 \mathrm{~kg} / \mathrm{kmol} ; \mu=$

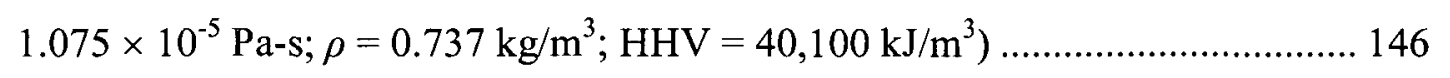

Table D.6 - Heavy 4-component fuel mixture test conditions $(M W=21.46 \mathrm{~kg} / \mathrm{kmol}$;

$$
\left.\mu=1.024 \times 10^{-5} \mathrm{~Pa}-\mathrm{s} ; \rho=0.883 \mathrm{~kg} / \mathrm{m}^{3} ; \mathrm{HHV}=46,900 \mathrm{~kJ} / \mathrm{m}^{3}\right) \ldots \ldots \ldots \ldots \ldots \ldots \ldots \ldots \ldots \ldots \ldots \ldots \ldots . . .146
$$

Table D.7 - Methane test conditions $\left(M W=16.043 \mathrm{~kg} / \mathrm{kmol} ; \mu=1.106 \times 10^{-5} \mathrm{~Pa}\right.$-s;

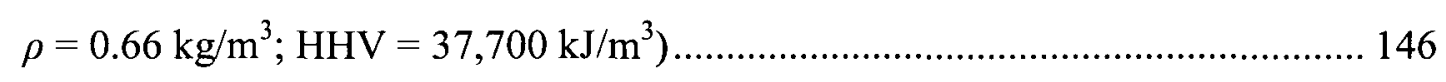




\section{List of Figures}

Figure 1.1 - Image from Google Earth of 2007 NPRI data for UOG Industry ................ 4

Figure 1.2 - Number of batteries and cumulative percentages of gas flared .................... 5

Figure 2.1 - A Pictorial approximation of the soot formation process [Richter and

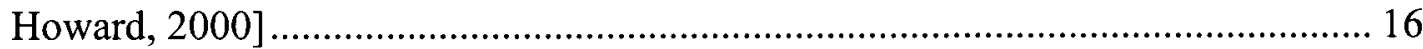

Figure 2.2 - Turbulent flame regimes identified by Delichatsios [1993a] ...................... 21

Figure 2.3 - Estimated flare regimes: red line represents a $76.2 \mathrm{~mm}$ (3") burner and the blue line represents a $254 \mathrm{~mm}(10$ ") burner. The range for each burner was estimated based on a minimum exit velocity of $0.1 \mathrm{~m} / \mathrm{s}$ (which gave values in the lower left of the shaded region) and a maximum exit velocity of $6 \mathrm{~m} / \mathrm{s}$ (which gave

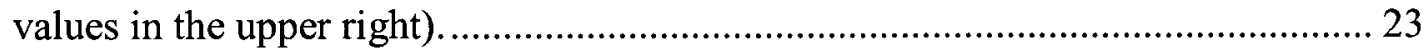

Figure 2.4 - Comparison of flame length theories 25

Figure 2.5 - Soot yield (displayed as a percentage) as a function of the mixing ratio as defined by Ellzey et al. [1990] for the data of Prybysh [2002] fitted to his exponential model and the power model of Ellzey et al. [1990]. Reproduced from Prybysh [2002].

Figure 2.6 - Comparison of flare regime with estimated regimes of available literature. 34

Figure 3.1 - Schematic of Lab Scale Flare (LSF) fuel delivery system, enclosure, and dilution tunnel.

Figure 3.2 - Schematic of the LSF [Canteenwalla, 2007]

Figure 3.3 - Mean, $10^{\text {th }}$, and $90^{\text {th }}$ Percentile Solution Gas Concentrations [private

communication between Dr. Matthew Johnson and PTAC, 2007] 39

Figure 3.4 - Light, Average, and Heavy 6-mix surrogate fuel mixtures 42

Figure 3.5 - Diagram of dilution tunnel and components

Figure 3.6 - Soot concentration traverse at fan speeds of (a) $15 \%$ and (b) $100 \%$. Error bars represent standard deviations of LII measured $f_{v}$. 46

Figure 3.7 - Diagram of Pitot probes and static ports. Radial probe locations in mm.... 48 Figure 3.8 - Velocity profiles in the horizontal and vertical directions at a fan speed of $100 \%$ 
Figure 3.9 - Schematic of Gravimetric sampling train.

Figure 3.10 - Schematic of filter holder and photograph of filter assembly, pictured here without the secondary dilution device (all dimensions in $\mathrm{mm}$ ). 53

Figure 3.11 - Photographs of filters deposited with small (left) and large (right) amounts of soot...... 55

Figure 3.12 - Mass of control filters with time. Key to symbols on Y-axes. Solid black lines represent the cumulative averages.

Figure 3.13 - Variation of blank filter 1 mass with (a) temperature and (b) relative humidity

Figure 3.14 - Schematic of Artium LII system [Canteenwalla, 2007]............................... 62

Figure 3.15 - Schematic of LII sampling train.

Figure 3.16 - Soot yield as a function of the DT fan speed for tests using the $25.4 \mathrm{~mm}$ burner using the average 6-component fuel mixture at 15.2 SLPM......................... 66 Figure 3.17 - Typical soot yield data for a test with the $25.4 \mathrm{~mm}$ burner using the average 6-component fuel at 15.2 SLPM 67

Figure 3.18 - Comparison of LII test (a) with and (b) without the points deemed outliers by Chauvenet's Criterion 68

Figure 4.1 - Soot yield from the $25.4 \mathrm{~mm}$ burner burning the AVG-6-component mixture measured using both the gravimetric technique and the LII method 84

Figure 4.2 - Soot yield for a range of burner diameters burning the AVG-6-component mixture measured using (a) the gravimetric technique and (b) the LII method...... 85

Figure 4.3 - Soot yield values for constant set point exit velocities for a range of burner diameters burning the AVG-6-component mixture measured using (a) the gravimetric technique and (b) the LII method.

Figure 4.4 - Soot yield values from the $25.4 \mathrm{~mm}$ burner burning the L-, AVG-, and H- 6and 4-component mixtures measured using (a) the gravimetric technique and (b) the LII method

Figure 4.5 - Soot yield at a constant set point exit velocity of $0.5 \mathrm{~m} / \mathrm{s}$ burning the L-, AVG-, and H- 6- and 4-component mixtures measured using (a) the gravimetric technique and (b) the LII method 
Figure 4.6 - Soot yield at a constant set point exit velocity of $0.25 \mathrm{~m} / \mathrm{s}$ burning the L-, AVG-, and $\mathrm{H}-4$-component mixture and the average 6-component mixture measured using (a) the gravimetric technique and (b) the LII method

Figure 4.7 - Comparison of soot yield measured using the LII and gravimetric techniques

Figure 4.8 - Measured visible flame lengths for all burner diameters and flow rates burning L-, AVG-, and H- (a) 6-component mixtures and (b) 4-component mixtures

Figure 4.9 - Comparison of measured visible flame length and calculated flame length. 92 Figure 5.1 - Comparison of current experimental results with Canteenwalla [2007] for the $25.4 \mathrm{~mm}$ burner burning methane .94

Figure 5.2 - Comparison of measured $S G E$ values for methane with methane data of

Becker and Liang [1982]. $R i_{L}=g L_{f}^{3}\left(u_{e} d_{e}\right)^{-2} \rho_{\infty} / \rho_{e}$ 96

Figure 5.3 - Relationship between global Froude number and Richardson ratio as measured using methane data.

Figure 5.4 - Regime map comparing current methane data points (crosses) and the region expected to include the data of Becker and Liang [1982] (dashed lines). 97

Figure 5.5 - Measured fuel temperatures $25 \mathrm{~mm}$ upstream of the burner exit for all burner diameters. 99

Figure 5.6 - Soot yield as a function of measured fuel temperature. 102

Figure 5.7 - Soot yield vs. mass flow rate for all diameters burning the AVG-6component fuel mixture. 104

Figure 5.8 - Regime map showing expected flare operation between red and blue lines, and current data for the AVG-6-component fuel mixture. 104

Figure 5.9 - Soot yield for all burners burning the AVG-6-component fuel mixture for the transition-buoyant sub-regime. 105

Figure 5.10 - Soot yield for all burners burning the AVG-6-component fuel mixture for the transition-shear sub-regime 106 
Figure 5.11 - Soot yield as a function of the fire Froude number for (a) all burners and (b) the three largest burners burning the AVG-6-component fuel.

$$
F r_{f}=u_{e} f_{s}^{3 / 2}\left(\rho_{e} / \rho_{\infty}\right)^{-1 / 4}\left(g d_{e} \Delta T_{f} / T_{\infty}\right)^{-1 / 2}
$$

Figure 5.12 - Soot yield as a function of the Richardson ratio as defined by Becker and Liang [1982] for (a) all burner diameters and (b) the three largest burner diameters burning the AVG-6-component fuel. $R i_{L}=g L_{f}^{3}\left(u_{e} d_{e}\right)^{-2} \rho_{\infty} / \rho_{e}$ 109

Figure 5.13 - Soot yield as a function of the global characteristic residence time $\left(\tau_{R}^{*}=\rho_{\infty} f_{s} L_{f}^{3} / \dot{m}_{\text {fiut }}\right)$ as defined by Becker and Liang [1982] for (a) all burner diameters and (b) the three largest burner diameters burning the AVG-6-component fuel. . 109 Figure 5.14 - Soot yield as a function of the buoyant residence time defined by Canteenwalla [2007] for (a) all burner diameters and (b) the largest three burner diameters burning the AVG-6-component fuel. $\tau_{R, B}=\left(-u_{e}+\sqrt{u_{e}^{2}+2 a_{B} L_{f}}\right) / a_{B} \ldots 110$ Figure 5.15 - (a) soot yield and (b) soot generation efficiency as a function of the fire Froude number for the $38.1 \mathrm{~mm}, 50.8 \mathrm{~mm}$, and $76.2 \mathrm{~mm}$ burners burning the fuel mixtures as identified in the legend. The ordinate scales are identical for both plots

Figure 5.16 - Smoke-point corrected (a) soot yield and (b) soot generation efficiency as a function of the fire Froude number for the $38.1 \mathrm{~mm}, 50.8 \mathrm{~mm}$, and $76.2 \mathrm{~mm}$ burners burning the fuel mixtures as identified in the legend. The ordinate scales are identical for both plots

Figure 5.17 - Regime map of all data for the three largest burners burning all fuel mixtures

Figure 5.18 - Soot yield as a function of the fire Froude number for the three largest burner diameters and all fuel mixtures. Hatched area is the transition region as determined from Figure 5.17

Figure 5.19 - Soot yield as a function of the Delichatsios scaling parameter for values with a $F r_{f}$ of (a) less than 0.003 and (b) greater than 0.003 . Lines represent linear fits of data. 
Figure 5.20 - Soot yield as a function of the adiabatic flame temperature determined using the NASA computer program CEA for values with a $F r_{f}$ of (a) less than 0.003 and (b) greater than 0.003. Lines represent linear fits of data .............................. 118

Figure 5.21 - Soot yield as a function of the volumetric heating value for (a) all $25.4 \mathrm{~mm}$ burner and (b) values with a $F r_{f}$ of greater than 0.003 . Lines represent linear fits of

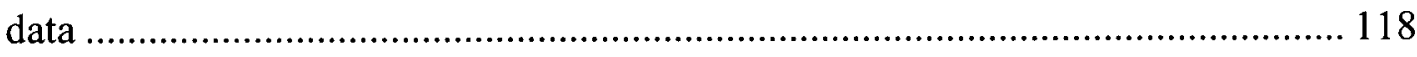

Figure 5.22 - Soot yield as a function of heating value for burners with diameters larger

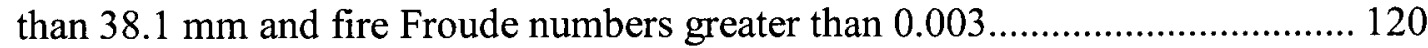

Figure $5.23-E F$ as a function of heating value for burners with diameters larger than $38.1 \mathrm{~mm}$ and fire Froude numbers greater than 0.003 121

Figure C.1 - Schematic of temperature measurement setup showing both the $12.7 \mathrm{~mm}$ and

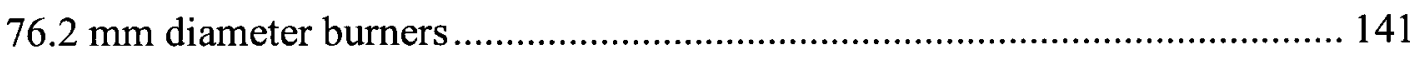




\title{
Nomenclature
}

\author{
Variables \\ A Area $\left[\mathrm{m}^{2}\right]$ \\ $a_{B} \quad$ Acceleration due to buoyancy $\left[\mathrm{m} / \mathrm{s}^{2}\right]$ (Equation 2.15, pg. 30) (cold flow \\ properties) \\ $A F_{s} \quad$ Stoichiometric air-to-fuel mass ratio \\ $B_{i} \quad$ Systematic uncertainty in $i$ \\ $c \quad$ Speed of light [299 $792458 \mathrm{~m} / \mathrm{s}]$ \\ $c_{p} \quad$ Specific heat $[\mathrm{J} / \mathrm{kg}-\mathrm{K}]$ \\ $d \quad$ Diameter $[\mathrm{m}]$ \\ $D a_{l} \quad$ First Damkohler number [s] \\ $D_{p} \quad$ Delichatsios parameter (Equation 5.1, pg. 116) \\ $D R \quad$ Dilution ratio (Equation 3.1, pg. 44) \\ $E\left(m_{\lambda}\right) \quad$ Absorption function used in LII analysis \\ $E F \quad$ NPRI emission factor $\left[\mathrm{kg}\right.$ soot $/ 10^{3} \mathrm{~m}^{3}$ fuel] (Equation 3.17, pg. 71) \\ $F \quad$ Radiation shape factor \\ $f_{s} \quad$ Stoichiometric mixture fraction (Equation 2.3, pg. 19) \\ $f_{v} \quad$ Soot volume fraction [ppb - parts per billion by volume] \\ Fr Froude number based on cold flow velocity $(F r=u / \sqrt{g d})$ \\ $F_{f} \quad$ Fire Froude number (Equation 2.6, pg. 20) (cold flow properties) \\ $\mathrm{Fr}_{g} \quad$ Global Froude number (Equation 2.4, pg. 20) (cold flow properties) \\ $F_{s} \quad$ Source Froude number (Equation 2.2, pg. 19) (cold flow properties) \\ g Gravitational constant $\left[9.81 \mathrm{~m} / \mathrm{s}^{2}\right]$ \\ GER Global equivalence ratio (Equation 2.14, pg. 30) \\ $G_{E X P} \quad$ Experimental detector gain used in LII analysis \\ $h \quad$ Convection heat transfer coefficient $\left[\mathrm{W} / \mathrm{m}^{2}-\mathrm{K}\right]$ \\ $h_{p} \quad$ Planck constant used in LII analysis [J·s] \\ $H \quad$ Height of fluid used in pressure measurements [m] \\ HV Volumetric heating value $\left[\mathrm{kJ} / \mathrm{m}^{3}\right]$
}




\begin{tabular}{|c|c|}
\hline$\Delta H_{c}$ & Heat of combustion $[\mathrm{kJ} / \mathrm{kg}]$ \\
\hline$k$ & Stefan-Boltzmann constant $\left[5.6704 \times 10^{-8} \mathrm{~W} / \mathrm{m}^{2}-\mathrm{K}^{4}\right]$ \\
\hline$k_{f}$ & Thermal conductivity [W/K-m] \\
\hline$L_{f}$ & Flame length $[\mathrm{m}]$ \\
\hline$m$ & Mass $[\mathrm{kg}]$ \\
\hline$\dot{m}$ & Mass flow rate $[\mathrm{kg} / \mathrm{s}]$ \\
\hline$M W$ & Molecular weight $[\mathrm{kg} / \mathrm{kmol}]$ \\
\hline$N u$ & Nusselt number (Equation C.5, pg. 143) \\
\hline$P_{i}$ & Precision uncertainty in $i$ \\
\hline$P_{d}$ & Dynamic pressure $[\mathrm{Pa}]$ \\
\hline$P_{s}$ & Static pressure $[\mathrm{Pa}]$ \\
\hline$P e$ & Peclet number (Equation C.4, pg. 143) \\
\hline $\operatorname{Pr}$ & Prandtl number $\left(c_{p} \mu / k_{f}\right)$ \\
\hline$Q$ & Volume flow rate $\left[\mathrm{m}^{3} / \mathrm{s}\right]$ \\
\hline$r$ & Radius [m] \\
\hline $\operatorname{Re}$ & Reynolds number ( $R e=\rho u d / \mu)$ (cold flow properties) \\
\hline R.H. & Relative humidity [\%] \\
\hline$R i$ & Richardson ratio $\left(R i=g L / u^{2}\right)$ (cold flow properties) \\
\hline$R i_{L}$ & $\begin{array}{l}\text { Flame length calculated Richardson ratio (Equation 2.9, pg. 28) (cold flow } \\
\text { properties) }\end{array}$ \\
\hline$R_{u}$ & Universal gas constant $[8314 \mathrm{~J} /(\mathrm{kmol} \cdot \mathrm{K})]$ \\
\hline$\dot{S}_{s p}$ & Smoke-point heat release rate (Equation 2.13, pg. 29) \\
\hline$S G E$ & $\begin{array}{l}\text { Soot generation efficiency [ } \mathrm{kg} \text { soot } / \mathrm{kg} \text { fuel bound carbon] (Equation } 2.8, \mathrm{pg} \text {. } \\
\text { 27) }\end{array}$ \\
\hline$T$ & Temperature $[\mathrm{K}]$ \\
\hline$T_{p}$ & Particle temperature used in LII analysis $[\mathrm{K}]$ \\
\hline$u$ & Velocity $[\mathrm{m} / \mathrm{s}]$ \\
\hline$U_{i}$ & Total uncertainty in $i$ \\
\hline$v$ & Degrees of freedom \\
\hline$V$ & Volume $\left[\mathrm{m}^{3}\right]$ \\
\hline
\end{tabular}




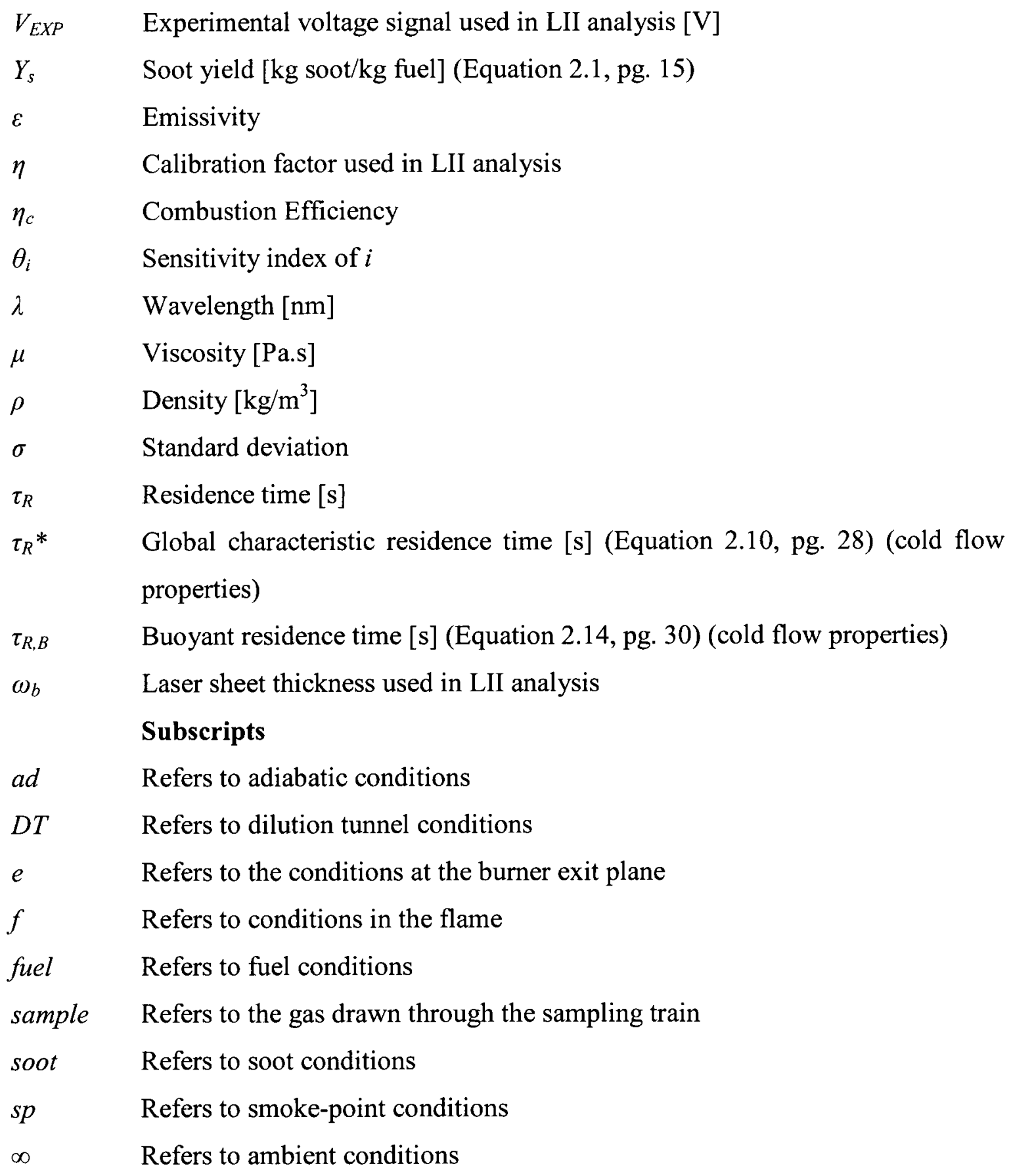




\begin{tabular}{ll} 
CFM & Cubic feet per minute \\
DT & Dilution tunnel \\
EC & Elemental carbon \\
EMC & Emission Measurement Centre \\
ERCB & Energy Resources Conservation Board of Alberta \\
ETP & Engine Test Procedures \\
F.S. & Full-scale \\
GHG & Greenhouse gas \\
GWP & Global warming potential \\
HP & Horsepower \\
I.D. & Inner Diameter \\
LDV & Laser Doppler Velocimetry \\
LII & Laser induced incandescence \\
LPM & Actual litres per minute \\
LSF & Lab-scale flare \\
MCE & Mixed cellulose ester \\
MFC & Mass flow controller \\
MSDS & Material Safety Data Sheets \\
NAICS & North American Industry Classification System \\
NEMA & National Electrical Manufacturers Association \\
NOX & Nitrogen oxides \\
NPRI & National Pollutant Release Inventory \\
NPS & Nominal pipe size \\
NRC & National Research Council of Canada \\
NRCan & Natural Resources Canada \\
O.D. & Outer Diameter \\
OC & Organic carbon \\
PAH & Polycyclic aromatic hydrocarbons \\
PM & Particulate matter \\
PM \\
P.5 & Particulate matter smaller than $2.5 \mu$ m \\
PTAC & Petroleum Technology Alliance of Canada \\
\hline
\end{tabular}


PTFE Polytetrafluoroethylene

SLPM Standard litres per minute (where standard conditions are: $101325 \mathrm{~Pa}, 273 \mathrm{~K}$ )

SMPS Scanning mobility particle sizer

TC Total carbon

TSI Threshold sooting index

UNECE United Nations Economic Commission for Europe

UOG Upstream oil and gas

USEIA United States Energy Information Agency

USEPA United States Environmental Protection Agency 


\section{Chapter 1}

\section{Introduction}

Flaring is the common practice of burning off unwanted, flammable gas in an open atmosphere flame. Flaring is used extensively in the upstream oil and gas (UOG) industry as well as in downstream petrochemical and other industrial processes. While the composition of flared gas can vary significantly, in general, the major constituent is methane. Since methane has a Global Warming Potential ${ }^{1}$ (GWP) 25 times higher than $\mathrm{CO}_{2}$ on a mass basis [IPCC, 2007], flaring significantly reduces greenhouse gas (GHG) emissions that would occur if the gas were simply vented to atmosphere. However, flaring can produce soot and other pollutant species that have negative effects on air quality and the environment [e.g. Pohl et al., 1986; Strosher, 2000]. Several of these emissions are monitored by the National Pollutant Release Inventory (NPRI), an agency of Environment Canada, but the methods of soot estimation (i.e. particulate matter, PM) are at best oversimplified and of questionable accuracy. This project was initiated in response to the critical need for improved methods to estimate soot emissions from

\footnotetext{
${ }^{1}$ calculated on a 100 year time-scale
} 
flaring activity and to enable improved prediction and management of their impact in Canada, and globally.

Current soot emissions estimates are derived from multiplicative emissions factors defined as mass of particulate matter produced per mass of fuel burned or per rate chemical energy release though combustion. As discussed in detail in Section 1.1.4 below, the recommended procedures for estimating soot for UOG flares [CAPP, 2007] are derived from data that may not be applicable to open-atmosphere flames, calling into question the accuracy of current soot, or $\mathrm{PM}_{2.5}{ }^{1}$, emissions estimates for flaring in Canada. This is a critical issue for responsible management of flaring given that soot has been implicated as a significant health hazard [Pope et al., 2002] that has been linked to serious, adverse cardiovascular, respiratory, reproductive, and developmental effects in humans [USEPA, 2008]. Furthermore, soot is also recognized as an important source of anthropogenic radiative forcing of the planet's surface [Hansen et al., 2000; Ramanathan and Carmichael, 2008]. A recent estimate [Bond et al., 2007] of annual releases of the black carbon (or elemental carbon - the most significant climate forcing component of soot [IPCC, 2007) from energy-related combustion was approximately $4400 \mathrm{Gg}$ in the year 2000. However it was not known how much flaring contributed to these global estimates.

\subsection{Flaring}

Gas is typically flared because it has been deemed uneconomic to process ${ }^{2}$ or it may be flared due to leakages, purges, or an emergency release of gas in a facility. The United

\footnotetext{
${ }^{1} \mathrm{PM}_{2.5}$ is defined as particulate matter with diameters less than $2.5 \mu \mathrm{m}$.

${ }^{2}$ i.e. if it is far from a gas pipeline or if it is 'sour' and thus includes sufficient amounts of toxic $\mathrm{H}_{2} \mathrm{~S}$ to make it uneconomic to process.
} 
States Energy Information Administration (USEIA) estimates that 102.8 billion $^{3}$ of gas were flared or vented worldwide in 2005 [USEIA, 2008]. Analysis of satellite imagery using visible light camera data suggests that global flaring rates may exceed 135 billion $\mathrm{m}^{3}$ annually [Elvidge et al., 2009]. Environment Canada has identified the province of Alberta as the most significant source of flaring in Canada [EC, 2005]. Alberta flaring data is available from the Energy Resources Conservation Board of Alberta (ERCB), which tracks the amount of gas flared and vented anually from the UOG industry in Alberta. Based on these data from the ERCB, Alberta's UOG industry flared approximately 0.723 billion $\mathrm{m}^{3}$ of gas in 2007 and 0.691 billion $\mathrm{m}^{3}$ of gas in 2008 [ERCB, 2009]. The Province of British Columbia flared an additional one-third of that amount or 0.234 billion $\mathrm{m}^{3}$ of gas in 2005 [BCOGC, 2008]. Data for other oil-producing provinces such as Saskatchewan, Newfoundland, and Nova Scotia were not publicly accessible.

Emission data reported to the NPRI is categorized by source category and province. Of the 3795 UOG sites $^{1}$ that reported to the NPRI in 2007, 3106 were located in Alberta $(\sim 82 \%), 336$ were located in British Columbia $(\sim 8.9 \%)$, and 332 were located in Saskatchewan $(\sim 8.5 \%)$. Thus, the NPRI data support the assumption that flaring from the UOG will be concentrated in Alberta. This is illustrated in Figure 1.1, a Google Earth image of a map developed by Environment Canada, showing the 2007 calendar year NPRI data for the UOG industry. This image depicts all UOG industry sites reporting to the NPRI, not just those reporting $\mathrm{PM}_{2.5}$ emissions.

\footnotetext{
${ }^{1}$ Defined as sites with the North American Industry Classification (NAICS) code 2111 - Oil and Gas Extraction. Note that reporting to NPRI is based on exceedance of various thresholds set by NPRI. As discussed later in this thesis, there are thousands more UOG facilities emitting PM than those currently reporting to NPRI.
} 
Of the 3106 UOG sites reporting data in Alberta, only $1231(\sim 40 \%)$ reported $\mathrm{PM}_{2.5}$ emissions, which includes emissions from all sources. The percentage of reporting flare sites which report $\mathrm{PM}_{2.5}$ is likely even lower than $40 \%$. This low percentage is possibly a consequence of the reporting threshold value assigned to $\mathrm{PM}_{2.5}$ emission of 0.3 tonnes per annum $^{1}$, which is approximately equivalent to a volume of $117,000 \mathrm{~m}^{3}$ of gas (calculated based on the current recommended emission factor as discussed below in Section 1.1.4). If an estimated release is less than the threshold reporting value, no data need be reported to the NPRI.

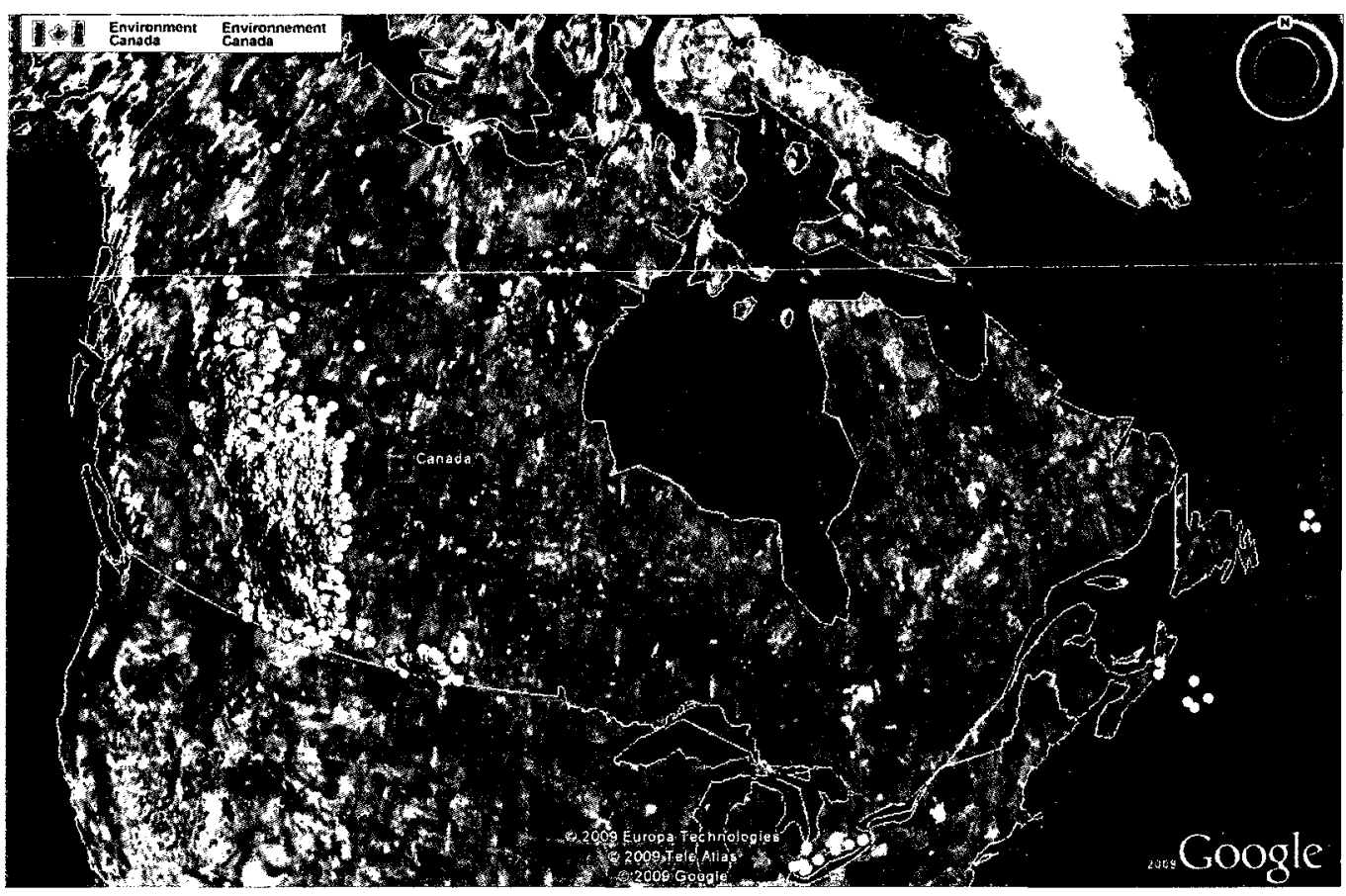

Figure 1.1 - Image from Google Earth of 2007 NPRI data for UOG Industry

\footnotetext{
${ }^{1}$ The threshold decreases to 0.15 tonnes for stacks that are greater than $50 \mathrm{~m}$ above grade. However, it is worth noting that stack height is not included in the NPRI database and that a typical stack height for a solution gas flare operating in the Alberta UOG industry is $10 \mathrm{~m}$ [Johnson et al., 2001a].
} 
Detailed data of solution gas flaring from crude oil and crude bitumen batteries ${ }^{1}$ has been made available to our research group by the ERCB [Private communication between Dr. Matthew Johnson and the ERCB]. Flaring from these batteries is the largest single contributor to flaring in the UOG industry in Alberta, representing approximately $48 \%$ of all gas flared in 2007 and 2008 [ERCB, 2009]. Figure 1.2 shows the approximately lognormal distribution of battery/flare volumes in Alberta. The NPRI threshold reporting volume for PM emissions is superimposed on the graph as a vertical blue line. These data suggest that approximately $74 \%$ of all oil and bitumen batteries that flare would not need to report $\mathrm{PM}_{2.5}$ data, unless reporting was triggered by threshold exceedance for another compound such as $\mathrm{NO}_{\mathrm{X}}$ or $\mathrm{CO}$ (although this is unlikely).

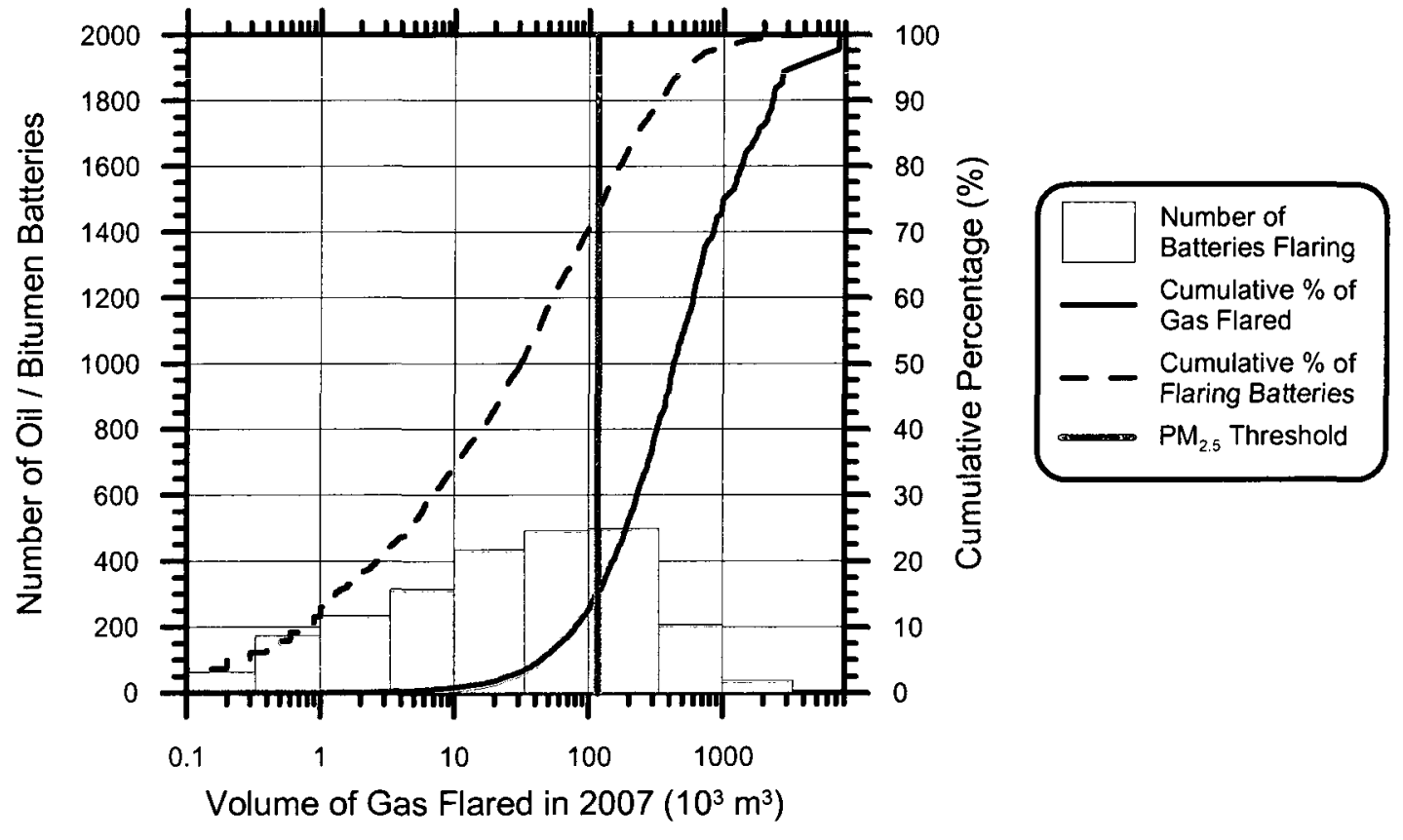

Figure 1.2 - Number of batteries and cumulative percentages of gas flared

\footnotetext{
${ }^{1}$ A battery is a typically small upstream facility that is fed by one or more wells, where primary separation of oil, water, and "solution gas" (a.k.a. associated gas) takes place. A battery typically has a single flare to process any surplus gas.
} 


\subsubsection{Categorization of Flares}

Flares can roughly be grouped into three main categories: emergency flares, process flares, and production flares [Johnson et al., 2001a]. Emergency flaring typically occurs when there is a safety concern for the plant personnel or the plant itself. Under an emergency condition (fire, compressor failure, valve rupture, over pressure, etc.), large volumes of gas may need to be disposed of quickly, and under these conditions the flow rates and exit velocities may be quite high. Process flares are typically used to dispose of gas at lower flow rates on a continuous or intermittent basis during normal processes at refineries and petrochemical plants. Production flaring can be further split into two categories: well testing and solution gas flaring. Well test flaring usually occurs during the development of a gas well, when gas may be flared at very high flow rates for a period of a few days. By contrast, solution gas flaring typically occurs when the gas produced during the installation of an oil well (i.e. solution gas) is deemed uneconomic to conserve, in which case flaring may continue for the life of the oil-well. Typically, solution gas flares operate at relatively low flow rates and exit velocities when compared to emergency, or well test flares. However, due to their overwhelming number and continuous emission, solution gas flares are the dominant source of flaring in the UOG industry in Alberta [Johnson et al., 2001a].

\subsubsection{Solution Gas Flares}

Solution gas is a term used to describe dissolved gas that is released from oil when its pressure is reduced from the higher reservoir pressure to atmospheric pressure. Once oil has been brought to the surface, it is separated from water and solution gas at a battery. 
The water is re-injected into the well and the solution gas may be conserved (i.e. used for on-site fuel or directed into a pipeline for processing and sale), flared, or vented.

A typical solution gas flare in Alberta nominally comprises a 4" $(101.6 \mathrm{~mm})$ inner diameter pipe stack $10 \mathrm{~m}$ in height [Johnson et al., 2001a]. This is in contrast to commercial flares (i.e. flares used in the manufacturing industry, petroleum refining, and landfill and wastewater applications), which are more complicated in design and could include a commercial burner tip, pilot, and smoke suppressant. Commercial burner tips can be complex designs including wind protection, steam injection, and pilot flame integration. The pilot, if present, is used to keep the flare lit on start-up and in case of low energy density or high crosswinds. There are two kinds of smoke suppressants commonly used in commercial flares: steam and compressed air. While there are many options available for improving flare performance, these are rarely, if ever, exercised with the smaller and often remotely-located UOG solution gas flares typically found in Alberta.

\subsubsection{Flare Efficiency and Soot}

Few published studies have appeared in the literature on actual emission rates of soot and overall efficiency from flares. Some of the earliest research dates to the early 1980 's with a series of reports commissioned by the USEPA [McDaniel, 1983]. In this work, samples were collected from a 8" (203.2 mm) diameter flare burning propylene with exit velocities from $2.3-4.2 \mathrm{~m} / \mathrm{s}$. The soot measurements made in this work were of exhaust gas soot concentration only, and since the dilution of the samples was not known, data were not directly relatable to fuel consumption. Using the same facility as McDaniel [1983], Pohl et al. [1986] studied the effect of soot on combustion efficiency from flares 
burning propane with burner diameters ranging from 3 " $(76.2 \mathrm{~mm})$ to 12 " $(304.8 \mathrm{~mm})$ and exit velocities ranging from $0.03 \mathrm{~m} / \mathrm{s}$ to $30 \mathrm{~m} / \mathrm{s}$. The effect of soot on combustion efficiency was studied as part of a larger project which was commissioned to establish the combustion efficiency of large-scale flares typical of downstream refineries or upstream well-tests, and to investigate if any physical characteristics had influences on the combustion efficiency. Specifically, they wanted to answer the following questions:

1. How does soot affect the measured combustion efficiency?

2. Can overall flare efficiencies be estimated from measurements at a single position?

3. How does the inability to close the mass balance on the products affect the measured combustion efficiency? (i.e. if soot is neglected, can efficiency still be determined from major gaseous species concentrations alone?).

4. How can the results be extrapolated to large-scale flares?

5. Which operating conditions result in low combustion efficiency?

The work was a collaborative project between the Energy and Environmental Research Corp. and the USEPA. The combustion efficiency, $\eta_{c}$, was awkwardly defined as the fraction of $\mathrm{CO}_{2}$ divided by the amounts of $\mathrm{CO}_{2}, \mathrm{CO}$, hydrocarbons, and soot in the dilution corrected combustion products. This work is relevant to the current research because they found that burner diameter and the presence of soot did not influence flare efficiency and "soot accounts for less than 0.5 percent of the unburned hydrocarbons emitted by flares" in most cases [Pohl et al., 1986]. They did observe that the volumetric heating value of the fuel had an effect on the combustion efficiency, where lower heating 
values led to less stable flames, resulting in lower combustion efficiencies. They also found that combustion efficiencies, using their definition, were generally greater than 98 percent.

Further to the work by Pohl et al. [1986], the work by Kostiuk et al. [2004] considered smaller scale flares with lower exit velocities more typical of solution gas flares found in the Alberta UOG industry. They examined the influence of cross winds on flare combustion efficiency and concluded that in general, at quiescent conditions, a flare would typically have combustion efficiencies (defined as the carbon conversion efficiency, i.e. the mass of carbon in produced $\mathrm{CO}_{2}$ divided by the mass of carbon in the hydrocarbon fuel) greater than 99.5 percent. This number would drop slightly with increasing crosswinds, and at high wind speeds would decrease dramatically due to a fuel stripping mechanism [Johnson and Kostiuk, 2000; Johnson et al., 2001b]. It was also found that crosswinds slightly decreased the amount of soot produced, in agreement with the work of Ellzey et al. [1990] who studied small-scale propane diffusion flames in cross-flow. The current research only focuses on quiescent wind conditions (i.e. zero cross-flow), which would represent the "worst case" sooting scenario.

\subsubsection{Emission Factors}

The threshold values discussed previously (Section 1.1) were calculated using the PM emission factor published in an NPRI guide developed by CAPP for use in the UOG industry [CAPP, 2007]. This guide provides several procedures to aid industry members in reporting emissions properly to the NPRI. It should be noted that the guide explicitly stipulates that "use of the report or any information contained will be at the user's sole risk," but recommends its use so emissions reporting will be both consistent and 
comparable between upstream oil and gas companies. For lack of alternative methods for estimating PM emissions from flares, it is likely that the method supplied in the guide is used widely. The guide specifies simple emission factors based on volumes of gas flared. The factor is published as $2.5632 \mathrm{~kg} \mathrm{PM}_{2.5} / 10^{3} \mathrm{~m}^{3}$ gas flared ${ }^{1}$. Given the wide variation in flare emissions associated with large variations in meteorological conditions, fuel composition, fuel flow rates, flare size, and flare design, this approach to estimating emissions is at best oversimplified.

Review of the cited sources in the CAPP NPRI guide reveals that the emission factor used to estimate production of soot from flares is based on a confidential report from the USEPA entitled "Data from flaring landfill gas" [USEPA, 1991]. The emission factor is published in USEPA's Factor Information Retrieval Data System (FIRE) database as $0.85 \mathrm{~kg}$ soot per $10^{3} \mathrm{~m}^{3}$ of fuel [USEPA, 2009]. The CAPP guide lists a value of $2.5632 \mathrm{~kg}$ soot per $10^{3} \mathrm{~m}^{3}$ of fuel, and notes that this value has been "corrected" for a gas with a heating value of $45 \mathrm{MJ} / \mathrm{m}^{3}$, which is typical of flare gas in the UOG industry. The CAPP guide does not specify how this apparent factor of three correction was derived, although it may have resulted from an assumed linear scaling of soot emission with heating value, since landfill gas could be expected to have a heating value on the order of $15 \mathrm{MJ} / \mathrm{m}^{3}$ based on the landfill gas composition work of Eklund et al. [1998]. The USEPA has also published emission factors for industrial flares [USEPA, 1995] derived from published research of McDaniel [1983].

Table 1.1 shows a comparison of emissions factors for flare-generated soot currently in use. As can be seen the emission factors vary by orders of magnitude. Of

\footnotetext{
${ }^{1}$ This number was used to back-calculate the amount of gas needed to produce $300 \mathrm{~kg}$ of $\mathrm{PM}_{2.5}$ to establish the threshold value mentioned in section 1.1.
} 
the three factors for flares reported in USEPA's WebFIRE database, the first two were largely derived from measurements on enclosed flares (although full details of the measurements are not publicly available) burning gas compositions ${ }^{1}$ that would have limited relevance to UOG flares. The third factor, as reported, is up to four orders of magnitude higher than the other two values. However, quite significantly, there is an apparent clerical error in the form of a swap of units from $\mu \mathrm{g}$ PM per $10^{-3} \mathrm{~m}^{3}$ exhaust gas to $\mathrm{lb}$ PM per $10^{6}$ BTU between the emission factor as reported in USEPA AP-42 [1995] and the value reported in USEPA WebFIRE [2009]; both are attributed to the same data source. The gas used is also reported incorrectly, as the fuel used in the cited sources of USEPA AP- $42^{2}$ is pure propylene, not the mixture mentioned in Table 1.1. The USEPA was contacted for background information on the factors as reported, and once confirmation was made that they were indeed reported as incorrect, it was brought to their attention. Unfortunately, contact with the USEPA has since ceased, and as of March 2009, the factor is still published incorrectly. Moreover, none of these factors are based on any measurements from actual solution gas flares (the CAPP guide value is derived from the $0.85 \mathrm{~kg}$ PM per $10^{3} \mathrm{~m}^{3}$ of fuel value from Web FIRE) and none give any consideration to operating conditions of a flare including wind speed, exit velocity, detailed fuel composition, flare size, or flare tip design, even though these parameters can significantly affect soot production. These discrepancies and simplifications bring into question the validity and credibility of the emission factors used for such a potentially

\footnotetext{
${ }^{1}$ Pure methane and landfill gas.

${ }^{2}$ [McDaniel, 1983].
} 
significant source of PM emissions ${ }^{1}$. The lack of accurate guidelines for NPRI reporting of soot from flares in the UOG industry is a primary motivation for the current work.

Table 1.1 - Comparison of Current Emission Factors

\begin{tabular}{|c|c|c|c|}
\hline Standard & $\begin{array}{l}\text { Original Emission } \\
\text { Factor as Reported }\end{array}$ & $\begin{array}{l}\text { Emission Factor (kg } \\
\text { PM per } 10^{3} \mathrm{~m}^{3} \text { of fuel) }\end{array}$ & Gas \\
\hline CAPP Guide [2007] & $\begin{array}{l}2.5632 \mathrm{~kg} \mathrm{PM} \mathrm{per} 10^{3} \mathrm{~m}^{3} \\
\text { fuel }\end{array}$ & $2.5632^{\mathrm{a}}$ & Solution Gas ${ }^{\mathrm{b}}$ \\
\hline \multirow{3}{*}{$\begin{array}{l}\text { USEPA WebFIRE } \\
\text { [2009] v. } 6.25\end{array}$} & $53 \mathrm{lb}$ PM per $10^{6} \mathrm{ft}^{3}$ gas & 0.85 & Landfill $\mathrm{Gas}^{\mathrm{c}}$ \\
\hline & $17 \mathrm{lb}$ PM per $10^{6} \mathrm{ft}^{3}$ gas & 0.27 & Methane \\
\hline & $0-274 \mathrm{lb}$ PM per $10^{6} \mathrm{BTU}$ & $5301^{d}$ & $\begin{array}{l}\text { 80\% Propylene, } \\
20 \% \text { Propane }^{\mathrm{e}}\end{array}$ \\
\hline $\begin{array}{l}\text { USEPA AP-42 [1995] } \\
\text { Vol. I, section } 13.5\end{array}$ & $\begin{array}{l}0-274^{\mathrm{f}} \mu \mathrm{g} \text { PM per } 10^{-3} \mathrm{~m}^{3} \\
\text { exhaust gas }\end{array}$ & $0,0.5,2.2,3.4^{\mathrm{g}}$ & $\begin{array}{l}80 \% \text { Propylene, } \\
20 \% \text { Propane }^{\mathrm{e}}\end{array}$ \\
\hline
\end{tabular}

${ }^{a}$ The excess significant figures in this emission factor are as reported in the CAPP Guide [2007].

${ }^{b}$ Solution gas typically contains a significant portion of methane, although the CAPP guide only specifies that the heating value is taken to be $45 \mathrm{MJ} / \mathrm{m}^{3}$.

${ }^{c}$ While the source for this emission factor is not publicly available and the composition of landfill gas varies, a "typical" composition could be $56 \%$ methane, $37 \% \mathrm{CO}_{2}, 1 \% \mathrm{O}_{2}$, and trace amounts of other gases [Eklund et al., 1998].

${ }^{\mathrm{d}}$ Based on a fuel heating value of $45 \mathrm{MJ} / \mathrm{m}^{3}(1 \mathrm{BTU}=1055.06 \mathrm{~J})$.

${ }^{\mathrm{e}}$ The fuel reported in these sources is incorrect. Review of the cited sources notes that the fuel is pure propylene [McDaniel, 1983].

i The range of $0-274$ is based on the "smoking level" of the flare: non-smoking flares, $0 \mu \mathrm{g} / \mathrm{L}$; lightly smoking flares, $40 \mu \mathrm{g} / \mathrm{L}$; average smoking flares, $177 \mu \mathrm{g} / \mathrm{L}$; and heavily smoking flares, $274 \mu \mathrm{g} / \mathrm{L}$.

${ }^{g}$ The method used to calculated these values are shown in Appendix A.

\subsection{Objectives}

The preceding discussion has clearly demonstrated the need for improved emission factors for estimating PM produced by solution gas flares. Since total emission measurements are very difficult to perform in the field, a predictive emission model based on field-measurable scaling parameters was desired. The goal of this research was to experimentally measure total soot emissions from the over-fire region of turbulent diffusion flames at conditions relevant to solution gas flares typical of the Alberta UOG industry. The test conditions were selected to scale to full-size flares based on fuel exit

\footnotetext{
${ }^{1}$ From Figure 1.2, approximately $85 \%$ of oil and bitumen batteries in Alberta flared volumes above the current NPRI reporting threshold for PM. Assuming this percentage applies to all flared gas sources and taking the CAPP PM emission factor at face value, then reportable $\mathbf{P M}_{2.5}$ from flared gas in Alberta represents $38.8 \%$ of all UOG reported $\mathrm{PM}_{2.5}$, or 1506 tonnes.
} 
velocity and burner size in order to match relevant flow regimes. The research was carried out as part of the Lab-Scale Flare (LSF) project at Carleton University in collaboration with the National Research Council of Canada (NRC) and with the support of Natural Resources Canada (NRCan) and CAPP. In summary, the main research objectives were to:

1. Modify the LSF facility to include a gravimetric sampling system and accommodate larger fuel flow rates

2. Develop a new sampling protocol for the new gravimetric and existing Laser Induced Incandescence (LII) sampling systems.

3. Use the upgraded facility and new sampling protocol to generate soot yield data for a wide range of operating conditions, including various fuel compositions, burner diameters, and fuel exit velocities

4. Investigate options for improved, yet practically implementable methods of predicting soot emissions based on fuel properties, burner size, and fuel flow rate

Chapter 2 outlines the relevant theory for soot production and scaling of turbulent diffusion flames. Details of the experimental approach, implementation, and data reduction are presented in Chapter 3. Results are presented in Chapter 4, followed by discussion in Chapter 5, and conclusions and recommendations in Chapter 6. 


\section{Chapter 2}

\section{A Brief Overview of Soot Formation Theory}

The soot formation process is very complex, involving several steps of chemical and physical growth and reduction [Appel et al., 2000; Bockhorn et al., 2002]. Although the mechanisms of soot formation are still not fully understood, key influencing parameters include fuel chemistry, fuel flow field, and temperature of the particles in the flame [Santoro and Semerjian, 1984; Kent and Wagner, 1984; Glassman, 1998]. Combining the complexities of soot formation and turbulent flame kinetics poses an extremely difficult problem. Most literature studies on soot formation have focused on laminar flames, and more specifically on the soot volume fraction inside the laminar flame as opposed to the total emission from the flame. While the spatially-resolved soot volume fraction inside a flame is important for understanding the soot formation process, the total soot emission is desired for emission reporting. The total soot yield, $Y_{s}$, is a useful measure of the soot emitted from the flame, as defined in equation 2.1 . 


$$
Y_{s}=\frac{\dot{m}_{\text {soot }}}{\dot{m}_{\text {fiel }}}
$$

where $\dot{m}_{\text {soot }}$ and $\dot{m}_{\text {fuel }}$ are the mass flow rates of soot and fuel, respectively. Recognizing the limitations imposed by the non-existence of a universal model to predict soot emission, the present work is focused on the development of a semi-empirical, experimental model for soot emission rates of diffusion flames based on basic flame characteristics.

\subsection{Formation of Soot}

The process of soot formation is summarized here based on the review by Richter and Howard [2000]. It is generally accepted that soot formation in a flame occurs in several steps as listed below and shown schematically in Figure 2.1.

1. Pyrolysis of fuel

2. Polycyclic Aromatic Hydrocarbon (PAH) formation

3. Formation of precursor particles

4. Particle inception

5. Surface growth and particle agglomeration

6. Particle oxidation

Soot production begins with the formation of molecular precursors that are thought to be heavy PAHs (typically benzene) [Frenklach and Warnatz, 1987]. These PAHs are formed after fuel pyrolysizes into small hydrocarbon radicals. Nucleation of particles from heavy PAH molecules subsequently occurs as mass is converted from molecular to particulate systems. The heavy PAH molecules form nascent soot particles with a 
diameter of approximately $1.5 \mathrm{~nm}$ [Richter and Howard, 2000]. These particles then grow as mass is added via the addition of gas phase molecules and/or other PAH molecules [Senkan and Castaldi, 1996; Smedley et al., 1992].

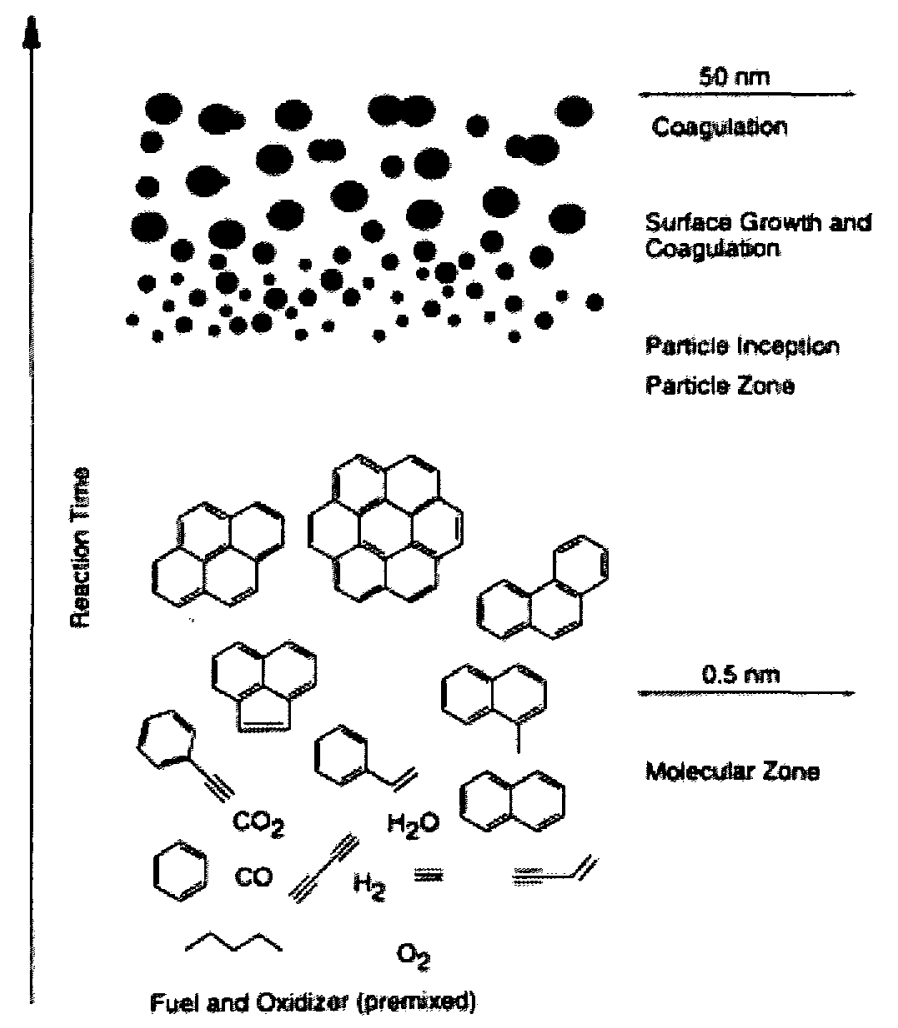

Figure 2.1 - A Pictorial approximation of the soot formation process [Richter and Howard, 2000]

The next steps in the process are coagulation and agglomeration, which occur via reactive particle-particle collisions, where particles bond with particles. This significantly increases the particle size and decreases the number of particles without changing the total mass of soot present in an agglomerate [Skjøth-Rasmussen et al., 2004; Richter and Howard, 2000; McKinnon and Howard, 1992]. Finally, oxidation decreases the mass of PAH and soot particles through reactions with oxygen radicals to form $\mathrm{CO}$ and $\mathrm{CO}_{2}$ [Neoh et al., 1982]. The final mass of soot emitted from a flame is the result of the competing effects of growth and oxidation, which are always ongoing throughout the flame. 


\subsection{Turbulent Jet-Diffusion Flames}

A diffusion flame (i.e. a non-premixed flame) is defined as a flame where the reactants of combustion (fuel and oxidizer) remain separate prior to combustion, but diffuse and mix together in the reaction zone. Turbulent jet-diffusion flames may be broadly classed into two categories depending on whether the turbulence is generated by instabilities present in the cold flow, or by the large buoyancy forces present in the flame [Delichatsios, 1993a]. The turbulence could be defined by the turbulence of the cold flow of fuel, but these instabilities may not carry through to the flame due to laminarization effects that could be present near the nozzle [Takagi et al., 1980]. Alternatively, a laminar cold flow could transition to a turbulent flame after the nozzle exit, where transition is affected either by buoyancy-induced instabilities or shear-laminar type instabilities at the burner exit.

In this sense, the physical flame can be broken into two zones as proposed by Delichatsios [1993a]: 1) a zone close to the nozzle exit referred to as the transition region, and 2) a zone encompassing the rest of the flame. These two zones can both be purely laminar, can both be purely turbulent, or the transition region can be laminar while the rest of the flame is turbulent.

The laminarization effect at the nozzle exit is attributed to the increase in kinematic viscosity due to the rapid temperature rise here. The associated decrease in density and associated density gradients drive the buoyancy-induced instabilities downstream in the flame [Takagi et al., 1980; Delichatsios, 1993a]. The "category" of turbulence regime generated will depend on the magnitude of the buoyancy forces to the inertia forces in the flame. This theory has led to the common use of a flame-derived Froude number, $F r$, in 
several studies on turbulent diffusion flames to define the flame regime as momentum- or buoyancy-driven. [e.g. Becker and Liang, 1978; Peters and Göttgens, 1991; Blake and McDonald, 1993; Delichatsios, 1993a]

An early such use was in the work of Becker and Liang [1978] when attempting to scale visible flame lengths of turbulent diffusion flames. They did not, however, develop the idea of a buoyancy parameter (Froude number); this was suggested by Homsy in 1969, whose results were unpublished. While Becker and Liang did not explicitly refer to this parameter as a Froude number, examination of the scaling parameter reveals that its definition is similar.

There exists a critical value of Froude number above/below which the flame will transition from buoyancy-driven to momentum-driven. The momentum-driven regime is defined as the regime where the flame length becomes independent of flow conditions. This is in stark contrast to the continually increasing flame length with Froude number of a buoyancy-driven flame. This is a well-documented trend, which was acknowledged as early as 1949 in the early flame length work of Hottel and Hawthorne [1949].

Depending on how the Froude number is defined, the transition value will vary. Within flame length related literature alone, several different forms of Froude number have been used, not including variations of the Richardson ratio, $R i^{1}$. One of the most common forms used is the source Froude number, $F r_{s}$ :

\footnotetext{
${ }^{1}$ The Richardson ratio is the square of the inverse of the Froude number.
} 


$$
F r_{s}=\frac{u_{e}}{\left(g d_{e}\right)^{1 / 2}}\left(\frac{\rho_{\infty}}{\rho_{e}}\right)^{1 / 4}
$$

where $u_{e}$ is the exit velocity, $g$ is the acceleration due to gravity, $d_{e}$ is the burner inner diameter, and $\rho_{\infty}$ and $\rho_{e}$ are the respective gas densities of the ambient air and of the fuel at the exit plane of the burner. For the current analysis, all fuel parameters (density, velocity, etc.) used in the calculation of this and all subsequent parameters were calculated at the cold flow conditions. To account for differences in stoichiometry, it has been suggested [Peters and Göttgens, 1991; Blake and McDonald, 1993; Delichatsios, 1993a] that the air-to-fuel ratio be included in the Froude number calculation. For diffusion flames, it is simple to replace the air-to-fuel ratio with the conserved scalar value of the stoichiometric mixture fraction, $f_{s}$, since the equivalence ratio is always close to unity as shown in Equation 2.3.

$$
f_{s} \equiv \frac{1}{A F_{s}+1}
$$

where $A F_{s}$ is the stoichiometric air-to-fuel mass ratio. Delichatsios [1993a] has suggested that this term be included raised to the power of $3 / 2$ as shown in Equation 2.4, which define a so-called global Froude number. Blake and McDonald [1993] suggest that this term be included raised to the power of 3, and Peters and Göttgens [1991] suggest that it should be included as the inverse of the square root. While these five authors disagree as to how the term should be included in the Froude number calculation, they at least agree it is important. This disagreement is one reason why there are many forms of the Froude number, each having unique buoyancy to momentum transition values. 


$$
F r_{g}=\frac{u_{e} f_{s}^{3 / 2}}{\left(g d_{e}\right)^{1 / 2}}\left(\frac{\rho_{\infty}}{\rho_{e}}\right)^{1 / 4}
$$

Independent of the value of the exponent attributed to $f_{\mathrm{s}}$, this general form of the Froude number still does not account for the buoyancy forces generated by the large rise in temperatures within the flame. Delichatsios [1993a] has suggested that a characteristic force per unit volume inside the flame plume at mean peak conditions be included, given by Equation 2.5 .

$$
\frac{\Delta \bar{\rho}_{f}}{\bar{\rho}_{f}} g=\frac{\Delta T_{f}}{T_{\infty}} g
$$

where $\Delta T_{f}$ is the characteristic temperature rise resulting from combustion calculated as $T_{a d}-T_{\infty}$, where $T_{a d}$ is the adiabatic flame temperature. This is combined into Delichatsios's final Froude number, termed the fire Froude number, $F r_{f}$, as shown in Equation 2.6. Through his experimental work, he determined that a flame will transition from buoyancy-dominated turbulence to momentum-dominated turbulence at a value of approximately $F r_{f}=5$.

$$
F r_{f}=\frac{u_{e} f_{s}^{3 / 2}}{\left(\frac{\rho_{e}}{\rho_{\infty}}\right)^{1 / 4}\left(\frac{\Delta T_{f}}{T_{\infty}} g d_{e}\right)^{1 / 2}}
$$

\subsubsection{Turbulent Regimes and Flares}

A critical aspect of attempting to compare results at different physical scales is the determination of the "regime" of a jet-diffusion flame. As discussed above, the turbulent regime has been loosely defined by Delichatsios [1993a] as being either buoyancy- or momentum-dominated. Turbulence in the buoyant regime is caused by large density gradients in the flame, which in turn cause instabilities and turbulence. By contrast, the 
turbulence in the momentum regime is caused by the turbulence inherent in the cold flow gas stream. Within the buoyant regimes, Delichatsios has identified other sub-regimes based on the mode of transition. The difference in generation of turbulence may be of importance when scaling flames.

Delichatsios [1993a] has identified these regimes graphically as is shown in Figure 2.2. The main regimes of interest in the present work were the turbulent buoyant (transition buoyant) and turbulent buoyant (transition shear) regimes. It should be noted that the abscissa in Figure 2.2 is the global Froude number, not the fire Froude number, and the ordinate is the cold flow Reynolds number. It is also noted that Delichatsios expected the transition between regimes to be smooth so that the lines in Figure 2.2 should not represent a sudden transition, and that the figure is only valid for hydrocarbon fuels. Equation 2.7 governs the line separating the two regimes of interest.

$$
\operatorname{Re}=1.46 F r_{g}^{-2}
$$

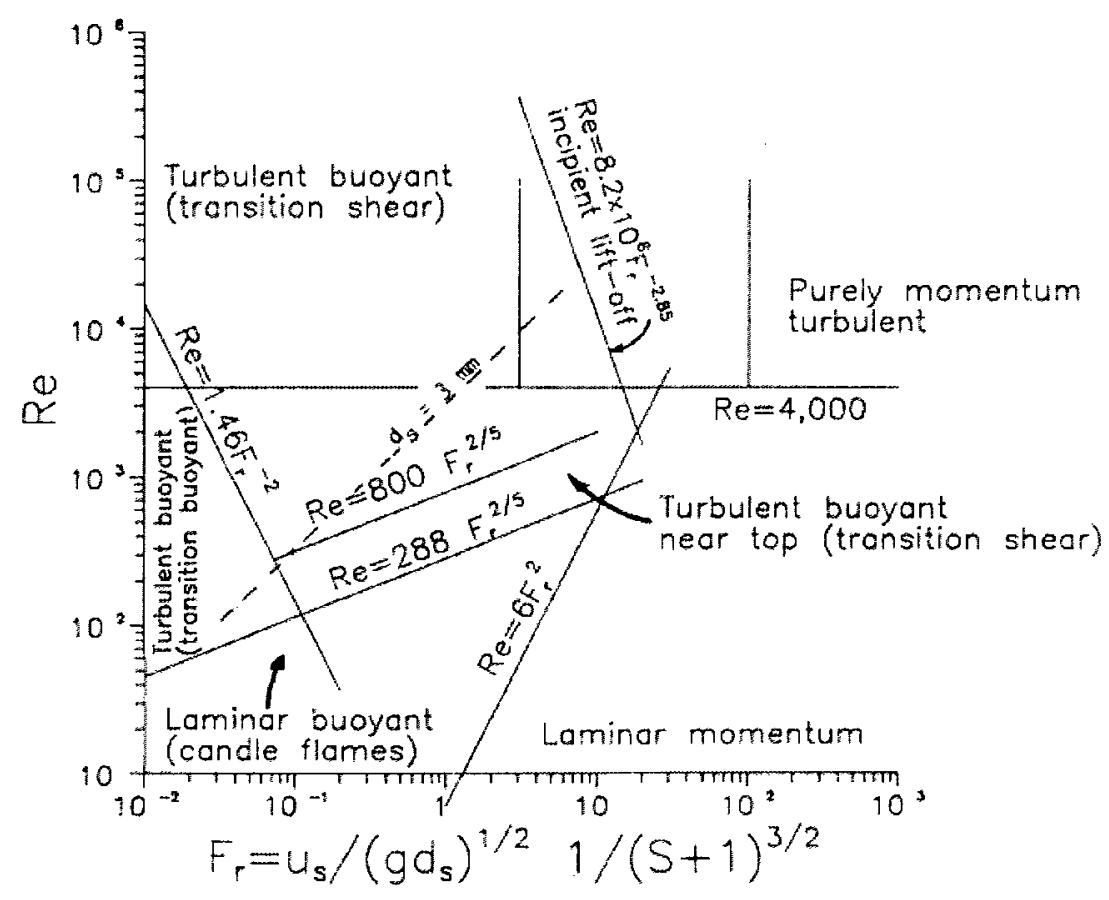

Figure 2.2 - Turbulent flame regimes identified by Delichatsios [1993a] 
The line defined by Equation 2.7 was developed based on the differences between the transition lengths at the burner exit. A transition that is dominated by buoyancy will exhibit a toroidal vortex near the exit and the transition length will be independent of flow rate and burner size. In contrast, an inertial transition exhibits sinusoidal instabilities and the transition length decreases as the nozzle exit velocity increases [Delichatsios, 1993a]. Anecdotally, this difference was visually observed in the present work while operating the LSF under different operating conditions.

To determine where solution gas flares fit into these regimes, and where test conditions should be set, the estimated conditions for solution gas flares representative of those found in Alberta were superimposed on the regime map of Delichatsios [1993a] (Figure 2.3). The Reynolds number and global Froude number were calculated based on fuel properties determined from data received from the Petroleum Technology Alliance of Canada (PTAC) [private communication between PTAC and Dr. Matthew Johnson, 2007], which will be discussed further in Chapter 3. Minimum and maximum expected flare diameters were estimated to be 3" $(76.2 \mathrm{~mm})$ and $10 "(254 \mathrm{~mm})$, with a 4" (101.6 mm) being "typical" as suggested by Johnson et al. [2001 a]. While it is extremely difficult to estimate actual flow rates and exit velocities typical of solution gas flares in Alberta, Johnson et al. [2001a], in an analysis of reported monthly volume data for approximately 4500 solution gas batteries in Alberta, suggested that typical exit velocities were less than $6 \mathrm{~m} / \mathrm{s}$, although this value is uncertain since it was not known if these flares were operating continuously or intermittently.

The estimated range of conditions expected for solution gas flares in Alberta is indicated by the hatched region in Figure 2.3. The bounding lower red line represents the 
$76.2 \mathrm{~mm}$ burner and the upper blue line represents the $254 \mathrm{~mm}$ burner. The range of exit velocities used was 0.1 to $6 \mathrm{~m} / \mathrm{s}$. As can be seen, according to the theory of Delichatsios [1993a], the range of flame conditions could all be classed as "turbulent-buoyant", although they span both the transition-buoyant and transition-shear sub-regimes. Experiments performed in the present work were therefore designed to span both subregimes under the assumption that the regime of the flame may impact the soot yield.

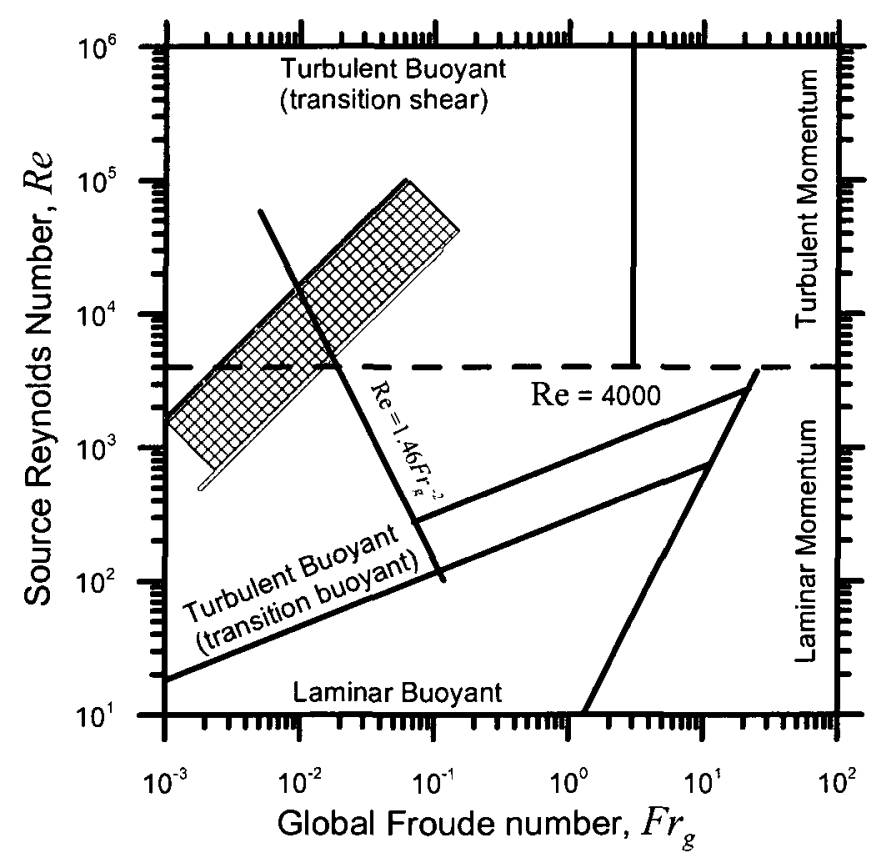

Figure 2.3 - Estimated flare regimes: red line represents a $76.2 \mathrm{~mm}$ (3") burner and the blue line represents a $254 \mathrm{~mm}\left(10^{\prime \prime}\right)$ burner. The range for each burner was estimated based on a minimum exit velocity of $0.1 \mathrm{~m} / \mathrm{s}$ (which gave values in the lower left of the shaded region) and a maximum exit velocity of $6 \mathrm{~m} / \mathrm{s}$ (which gave values in the upper right).

\subsubsection{Flame Length}

Flame length is a well-studied parameter of jet-diffusion flames that is relatively simple to measure visually. When combined with some sort of estimate for the characteristic velocity through the flame or velocity profile, the flame length can be used to give an accurate estimate of a particle's residence time, $\tau_{R}$, in the flame. The residence time of soot particles in a flame has been hypothesised to correlate with soot emission, as the 
residence time of the particle would represent the amount of time spent in the reaction zone where competing growth and oxidation reactions are taking place. It is thought that there should be some residence time of maximum soot yield, i.e. the soot particle exits the flame after undergoing maximum growth, but before being oxidized extensively. Sivathanu and Faeth [1990] have shown that soot yield values increase with residence time but become constant at some transition, suggesting that after the transition competing soot particle growth and oxidation rates negate each other.

Hottel and Hawthorne [1949] were among the first researchers to suggest a flame length correlation for flames in the momentum regime. Becker and Liang [1978] developed a theory for flame length estimation in both the buoyant and momentum regions based on their buoyancy parameter, which is similar to Equation 2.2 aside from the fact that the density ratio is raised to the power of 1 as opposed to $1 / 4$. Blake and McDonald [1993] also offered a theory based on dimensional analysis for the buoyant regime. Their work differed in that they calculated a theoretical lateral flame dimension, or flame width, to non-dimensionalize the flame length, where most others used the burner exit diameter. Peters and Göttgens [1991] provided approximate solutions to the flame length scaling laws by means of an analytical approach based on simplified treatment of the governing equations, i.e. continuity, momentum in the vertical direction, integrated radial momentum, and the mixture fraction. The downside to their approach is that it required several empirical constants that were different for different fuels. Delichatsios [1993a] provided a robust solution for flame length estimation in both the buoyant and momentum regimes based on the fire Froude number defined in Section 2.2. 
The three theories of Blake and McDonald, Peters and Göttgens, and Delichatsios were compared to assess their level of agreement. The calculations were done for burners of $12.7 \mathrm{~mm}$ and $76.2 \mathrm{~mm}$, and exit velocities ranging from $0.1 \mathrm{~m} / \mathrm{s}$ to $3 \mathrm{~m} / \mathrm{s}$. Fuel properties (densities, viscosity, stoichiometry, flame temperature) were based on data from PTAC [private communication between PTAC and Dr. Matthew Johnson, 2007] to be discussed further in chapter three. The results are displayed in Figure 2.4 below. The models of Blake and McDonald and Delichatsios show. reasonable agreement, but the flame length predictions from Peters and Göttgens are $16.4 \%$ shorter. This may be attributed to the fact that the empirical constants used by Peters and Göttgens were for propane since no other information was available, while the other calculations were based on more specific fuel properties. The work of Delichatsios includes robust theories for both the momentum and buoyant regimes; his work was used to compare the measured flame length data that accompanied soot yield measurements in the present work.

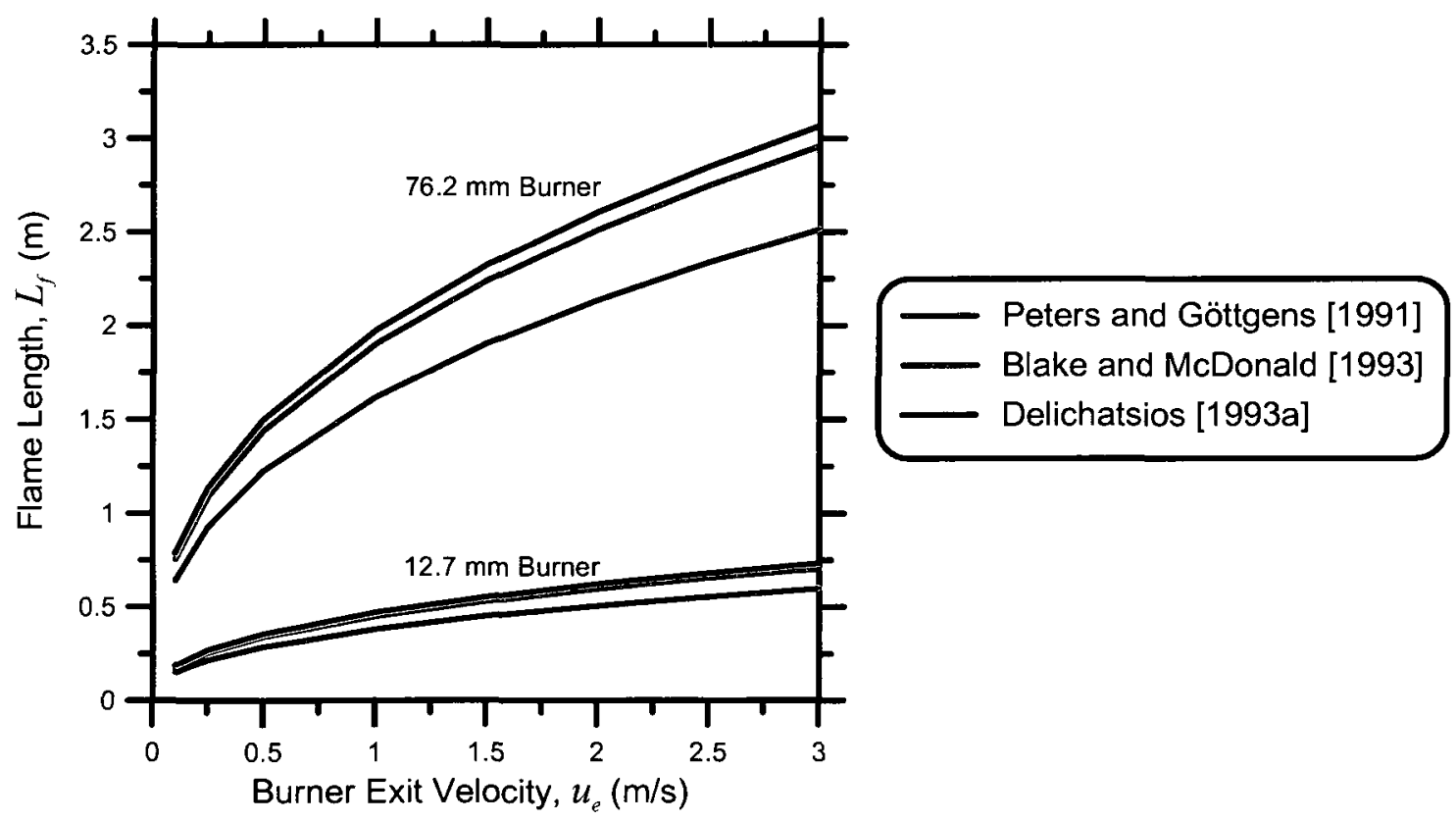

Figure 2.4 - Comparison of flame length theories 


\subsection{Soot Emissions from Turbulent Diffusion Flames and Flares}

There is a wealth of information in the literature on soot in laminar diffusion flames, but much less for turbulent diffusion flames. Work on laminar flames has typically focused on developing analytical models to predict soot formation and developing comparative indices for different fuels. The most common of these indices is the so-called smokepoint measurement, which is determined experimentally by increasing the fuel flow-rate until the tip of the laminar flame opens up (forming soot "wings") and smoke is observed to escape from the tip [Turns, 2000]. The smoke-point is usually referred to by the flow rate or the corresponding flame length observed at the smoke-point condition.

Several authors, [e.g. Kent, 1986; Schalla and McDonald, 1953] have provided a set of smoke-point measurements for a large number of gaseous and liquid fuel laminar flames. Calcote and Manos [1983] and Olson et al. [1985] instead suggested a threshold sooting index (TSI) for evaluating soot formation, calculated based on the smoke-point and molecular weight of the fuel with some experimentally-determined coefficients used to correct for differences in the amount of air required to burn a given quantity of different fuels. The value of TSI is then normalized to span a range of $0-100$, where the minimum measured TSI (corresponding to ethane) is assigned a value of 0 and the maximum measured TSI (corresponding to naphthalene) is assigned a value of 100 . The smoke-points and TSI values relevant to the current work are listed in Table 2.1. Although TSI and smoke-point data for laminar flames cannot be directly used to predict soot emissions from turbulent flames, they can provide a relative estimate of the variation attributable to fuel chemistry effects. 
Table 2.1 - Comparison of relevant sooting tendency measurements

\begin{tabular}{|c|c|c|c|c|}
\hline Fuel & $\begin{array}{c}\text { Smoke-point (mg/s) } \\
\text { [Kent, 1986] }\end{array}$ & $\begin{array}{c}\text { Smoke-point (mg/s) [Schalla } \\
\text { and McDonald, 1953] }\end{array}$ & $\begin{array}{c}\text { TSI [Calcote and } \\
\text { Manos, 1983] }^{\mathrm{a}}\end{array}$ & $\begin{array}{c}\text { TSI [Olson } \\
\text { et al., 1985] }\end{array}$ \\
\hline Methane & - & $34.53^{\mathrm{b}}$ & - & - \\
\hline Ethane & - & 16.52 & 0 & 1.5 \\
\hline Propane $^{\mathrm{c}}$ & 7.87 & 10.15 & 1.3 & 1.7 \\
\hline Butane $^{\mathrm{c}}$ & 7.00 & 8.6 & 1.6 & 2.0 \\
\hline
\end{tabular}

${ }^{a}$ TSI values were calculated based on data of Schalla and McDonald [1953].

${ }^{b}$ Value was calculated from a fifth order polynomial curve fit of Schalla and McDonald [1953] data for alkane fuels of $\mathrm{C} 2-\mathrm{C} 9$.

${ }^{c}$ Assumed n-butane.

\subsubsection{Turbulent, Vertical, Free Jet-Diffusion Flames}

Total soot emission from turbulent flames has been studied in the past [e.g. Becker and Liang, 1982; Sivathanu and Faeth, 1990; Delichatsios, 1993b]. However, most of these works typically considered pure fuels composed of heavier sooting alkene or alkyne hydrocarbons. Where alkanes have been studied, it has typically only been for propane. Becker and Liang [1982] studied the soot emissions from the alkane family of fuels more thoroughly. To the present author's knowledge, their data are the only measurements of the total soot emission from methane, ethane, and propane ${ }^{1}$ diffusion flames in the literature. Rather than the soot yield, Becker and Liang [1982] presented their results in terms of the soot generation efficiency ( $S G E$ ) defined according to Equation 2.8:

$$
S G E=\frac{\dot{m}_{\text {soot }}}{\dot{m}_{\text {carbon }}}
$$

where $\dot{m}_{\text {soot }}$ and $\dot{m}_{\text {carbon }}$ are the mass flow rates of soot and fuel carbon, respectively. This factor could be expected to better describe the conversion of carbon to soot for different fuels. Becker and Liang showed that while they could not develop a theory for scaling the $S G E$, they were able to show that the values changed under different flow conditions. These differing flow conditions were identified by a Richardson ratio $\left(R i_{L}\right)$,

\footnotetext{
${ }^{1}$ Also studied were hydrogen, CO, ethene, and ethyne (or acetylene).
} 
as defined in Equation 2.9. As can be seen, there is a heavy dependence of $R i_{L}$ on the flame length, and if flame length values vary slightly, large discrepancies in calculated $R i_{L}$ values can appear. Becker and Liang [1982] also defined a global characteristic residence time of material in a flame, $\tau_{R}{ }^{*}$, according to Equation 2.10. This residence time is analogous to the first Damkohler number, $D a_{1}$, and the $S G E$ is found to follow a trend for this residence time similar to that of the $R i_{L}$. Both of these terms will be investigated further in Chapter 5 as a potential means to correlate the data collected in the present work.

$$
\begin{gathered}
R i_{L}=\frac{g L_{f}^{3}}{\left(u_{e} d_{e}\right)^{2}}\left(\frac{\rho_{\infty}}{\rho_{e}}\right) \\
\tau_{R}^{*}=\frac{\rho_{\infty} f_{s} L_{f}^{3}}{\dot{m}_{\text {fivel }}}
\end{gathered}
$$

Sivathanu et al. [1988] recorded measurements of the soot volume fraction inside and above turbulent diffusion flames. While their results were in fair agreement with the work of Becker and Liang [1982], they did not provide any new correlation parameters. The work of Sivathanu and Faeth [1990] showed some simple correlations of the measured SGE with a smoke-point normalized residence time, $\bar{\tau}_{s p}$, as defined in Equation 2.11:

$$
\bar{\tau}_{s p}=\frac{\tau_{R}}{\tau_{s p}}
$$

where $\tau_{R}$ is the measured residence time and $\tau_{s p}$ is the measured smoke-point residence time.

Sivathanu and Faeth [1990] had defined residence time as the time interval between the interruption of the fuel flow, and the disappearance of all flame luminosity. The 
interruption was caused by rapidly moving a shutter across the exit of the burner. While the current experimental setup did not permit a duplication of this particular measurement, their work highlights the importance of the residence time in the literature.

In the work of Delichatsios [1993b], simple soot yield measurements were made for several fuels, including propane. He presented a reasonable correlation for the scaling of soot yield with a calculated smoke-point heat release rate and stoichiometric ratio shown in Equation 2.12. The smoke-point heat release rate was calculated according to Equation 2.13:

$$
\begin{gathered}
Y_{s} \sim \dot{S}_{s p} f_{s} \\
\dot{S}_{s p}=\dot{m}_{f i u l, s p} \Delta H_{c}
\end{gathered}
$$

where $\dot{m}_{\text {fuel,sp }}$ is the mass flow rate at the smoke-point and $\Delta H_{c}$ is the heat of combustion. While the heat release is an easy parameter to monitor, the smoke-point heat release is difficult to calculate for multi-component fuel mixtures. Compounding this problem is the lack of measurements of the smoke-point for methane, since the flame becomes unstable before it starts to smoke [Turns, 2000].

Glassman [1998] has suggested that the flame temperature and length of time that soot particles reside at these elevated temperatures will have an effect on the soot formation. Glassman postulated that what controls the soot volume fraction that exits the flame and causes soot emission is the distance between the isotherms that specify the incipient particle formation temperature and stoichiometric flame temperature (indicative of the strength of the temperature gradient). This distance establishes the growth time of the particles formed before flame oxidation of the soot occurs. While a universal theory 
for soot emission as a function of some temperature parameter is not given, it highlights the importance of the flame temperature on the soot formation.

Another work on soot yield was completed by Ouf et al. [2008], which focused on the effects of over-ventilating the flame. Specifically they were interested in changes in the size distributions of the primary particles and soot aggregates, morphology, and soot yield. To quantify the amount of ventilation, they proposed a global equivalence ratio, GER, as shown in Equation 2.14.

$$
G E R=\frac{A F_{s}}{A F_{\text {experiment }}}
$$

where $A F_{\text {experiment }}$ is the air-to-fuel ratio of the entire sampling system. While this is a useful parameter for scaling in an enclosed system, it is not directly applicable to open flames. Specifically, Ouf et al. [2008] found that the global equivalence ratio strongly influences the soot particle size, but does not play a predominant role in other soot morphological properties or emission.

Canteenwalla [2007] recorded measurements of soot yield from pure and simple mixtures of methane, ethane, and propane in the first iteration of the LSF project. Recognizing the importance of a residence time scaling parameter in the literature, he used a constant acceleration equation of motion to develop a buoyant residence time. This buoyant residence time calculation is shown in Equation 2.14; the buoyant acceleration is shown in Equation 2.15.

$$
\begin{gathered}
\tau_{R, B}=\frac{-u_{e}+\sqrt{u_{e}^{2}+2 a_{B} L_{f}}}{a_{B}} \\
a_{B}=\left(\frac{T_{a d}-T_{\infty}}{T_{\infty}}\right) g
\end{gathered}
$$


A smoke-point-normalized fuel mixture flow rate was developed by Canteenwalla [2007] in an attempt to collapse multiple SGE measurements of binary mixtures into a single curve. The results were promising, but did not work for mixtures that exhibited a synergistic effect, where the measured SGE was higher for the mixture than would be produced by either pure fuel. In his work, the methane/ethane mixtures produced the synergistic effect, thought to be the result of an interaction between a methyl radical $\left(\mathrm{CH}_{3}\right)$ and ethylene $\left(\mathrm{C}_{2} \mathrm{H}_{4}\right)$ which form a vinyl radical $\left(\mathrm{C}_{2} \mathrm{H}_{3}\right)$ that leads to an acetylene-based pathway to soot formation as hypothesized by Trottier et al. [2007].

\subsubsection{Turbulent Diffusion Flames in Cross-Flow Conditions}

Flares in the UOG industry are open to the air and it is expected that these flares will frequently be subjected to cross-flow wind conditions. The most relevant study of soot emissions from cross-flow flames was completed by Ellzey et al. [1990]. Prybysh [2002] also conducted limited studies of soot emissions from jet-diffusion flames in cross-flow, although these results were not published other than in his M.A.Sc. thesis. Both Ellzey et al. [1990] and Prybysh [2002] studied soot yields from turbulent propane flames under cross-flows in a wind tunnel for various burner exit diameters. Ellzey et al. [1990] concluded that except at very low cross-flow conditions, $\sim 0.7 \mathrm{~m} / \mathrm{s}$, the cross-flow conditions produced less soot than the same condition with a co-flow. Similar trends were reported by Prybysh [2002]. Ellzey et al. [1990] attributed the reduction in soot with increased cross-flow velocities to enhanced mixing and showed that soot yield correlated with the product of the exit velocity and cross-flow velocity.

The study of Ellzey et al. [1990] is important in that it identifies the cross-flow as reducing the total soot emission from turbulent diffusion flames. The magnitude of this 
dependence is illustrated in Figure 2.5, which plots data from both Prybysh [2002] and Ellzey et al. [1990], where the soot yield (displayed as a percentage) is shown to have an exponential decay with increasing mixing ratio, defined as the product of the fuel jet exit velocity and crosswind velocity. While flares will be subjected to crosswind in practice, the work of this thesis details only the soot emissions at quiescent conditions. In this sense, measurements made in this study will represent the "worst case" scenario since any cross-flow would reduce the total soot emission.

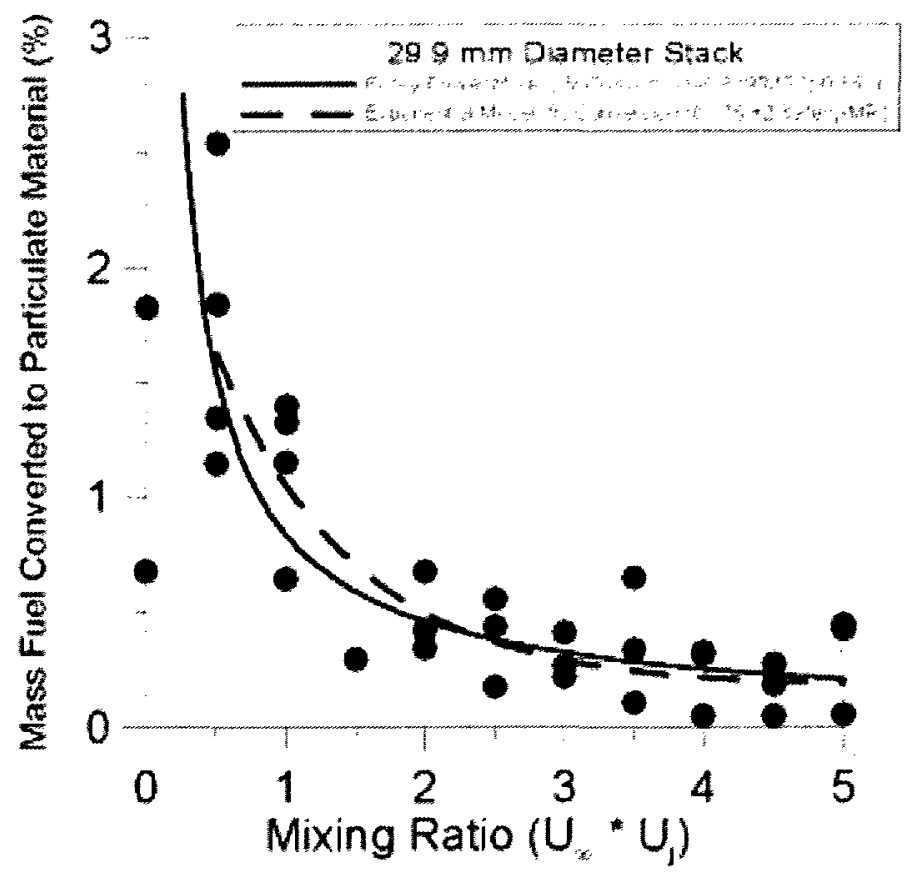

Figure 2.5 - Soot yield (displayed as a percentage) as a function of the mixing ratio as defined by Ellzey et al. [1990] for the data of Prybysh [2002] fitted to his exponential model and the power model of Ellzey et al. [1990]. Reproduced from Prybysh [2002].

\subsubsection{Flares}

Soot measurements have been made from an industrial scale flare with an exit diameter of $203.2 \mathrm{~mm}$ burning propylene with exit velocities from $2.3-4.2 \mathrm{~m} / \mathrm{s}$ by McDaniel [1983]. The soot measurements were taken post-flame by a probe just above the flame tip. The oxygen concentration was not measured in the sampled gas stream, so the 
measured soot concentrations could not be converted to the more useful soot yields because the amount of dilution was unknown. These measurements have been converted to soot yields for comparison by assuming an undiluted sample and complete combustion as detailed in Appendix A. The work of Pohl et al. [1986] measured soot concentrations, but did not provide any useful data in terms of concentrations or soot emission factors.

\subsection{Summary of soot measurement data}

Soot data from the relevant sources discussed above are listed below in Table 2.2. If values were published as $S G E$, they have been converted to $Y_{s}$. Global Froude numbers were estimated where sufficient information was provided.

Table 2.2 - Summary of relevant soot data

\begin{tabular}{|c|c|c|c|c|c|c|}
\hline Source & Fuel & $\begin{array}{c}\text { Range of } d_{e} \\
(\mathbf{m m})\end{array}$ & $\begin{array}{l}\text { Range of } u_{\varepsilon} \\
(\mathrm{m} / \mathrm{s})\end{array}$ & $\begin{array}{c}\text { Range of } \\
F r_{g}\end{array}$ & Estimated Regimes & $\begin{array}{c}\text { Range of } \\
Y_{s}\end{array}$ \\
\hline \multirow{3}{*}{$\begin{array}{l}\text { Becker and } \\
\text { Liang [1982] }\end{array}$} & Methane & $2.5-10.9$ & $13-75$ & $\begin{array}{l}0.89- \\
6.96\end{array}$ & $\begin{array}{l}\text { Turbulent buoyant, transition } \\
\text { shear; turbulent momentum }\end{array}$ & $\begin{array}{l}0.000882- \\
0.00214\end{array}$ \\
\hline & Ethane & $2.5-20.9$ & $9-62$ & $\begin{array}{l}0.53- \\
4.94\end{array}$ & $\begin{array}{l}\text { Turbulent buoyant, transition } \\
\text { shear; turbulent momentum }\end{array}$ & $\begin{array}{l}0.000401- \\
0.00200\end{array}$ \\
\hline & Propane & $0.7-10.9$ & $8-127$ & $\begin{array}{l}0.66- \\
19.76\end{array}$ & $\begin{array}{l}\text { Turbulent buoyant, transition } \\
\text { shear; turbulent momentum }\end{array}$ & $\begin{array}{l}0.000392- \\
0.00575\end{array}$ \\
\hline $\begin{array}{l}\text { Sivathanu and } \\
\text { Faeth [1990] }\end{array}$ & Propane & $50-234$ & $0.0063-0.1448$ & $\begin{array}{l}0.0001- \\
0.0027 \\
\end{array}$ & $\begin{array}{l}\text { Turbulent buoyant, transition } \\
\text { buoyant }\end{array}$ & $\begin{array}{l}0.007960- \\
0.01710\end{array}$ \\
\hline $\begin{array}{l}\text { Delichatsios } \\
\text { [1993b] }\end{array}$ & Propane & - & - & - & - & 0.00820 \\
\hline $\begin{array}{l}\text { Ellzey et al. } \\
\quad[1990]\end{array}$ & Propane & 216 & $0.702-1.671$ & $\begin{array}{l}0.19- \\
0.46\end{array}$ & $\begin{array}{c}\text { Turbulent buoyant, transition } \\
\text { shear }\end{array}$ & $\begin{array}{l}0.08600- \\
0.09190\end{array}$ \\
\hline $\begin{array}{l}\text { McDaniel } \\
\text { [1983] }\end{array}$ & Propylene & 203.2 & $2.3-4.2$ & $\begin{array}{l}0.02 \\
0.036\end{array}$ & $\begin{array}{c}\text { Turbulent buoyant, transition } \\
\text { shear }\end{array}$ & $\begin{array}{l}0.00050- \\
0.00337\end{array}$ \\
\hline \multirow{3}{*}{$\begin{array}{c}\text { Canteenwalla } \\
{[2007]}\end{array}$} & Methane & $25.4-50.8$ & $0.134-1.796$ & $\begin{array}{l}0.0028- \\
0.052\end{array}$ & $\begin{array}{l}\text { Turbulent buoyant, transition } \\
\text { buoyant } \& \text { transition shear }\end{array}$ & $\begin{array}{l}0.00001- \\
0.00011\end{array}$ \\
\hline & Ethane & $12.7-50.8$ & $0.045-4.991$ & $\begin{array}{l}0.0008- \\
0.18\end{array}$ & $\begin{array}{c}\text { Turbulent buoyant, transition } \\
\text { buoyant \& transition shear }\end{array}$ & $\begin{array}{l}0.00020 \\
0.00100\end{array}$ \\
\hline & Propane & $12.7-50.8$ & $0.008-4.286$ & $\begin{array}{l}0.0001- \\
0.16\end{array}$ & $\begin{array}{l}\text { Turbulent buoyant, transition } \\
\text { buoyant } \& \text { transition shear }\end{array}$ & $\begin{array}{l}0.00270- \\
0.00600\end{array}$ \\
\hline
\end{tabular}

Comparing propane between the works cited above, the soot yield varies from $3.92 \times 10^{-4}$ to $9.190 \times 10^{-2} \mathrm{~kg}$ soot $/ \mathrm{kg}$ fuel, a range of two orders of magnitude. Figure 2.6 displays the estimated regime ranges for several literature sources and shows that none of the cited literature is in the estimated regime of solution gas flares. The limited 
total soot emission data available in the literature and the lack of total soot emission data for fuel mixtures relevant to solution gas flaring in Alberta highlight the need for the present work.

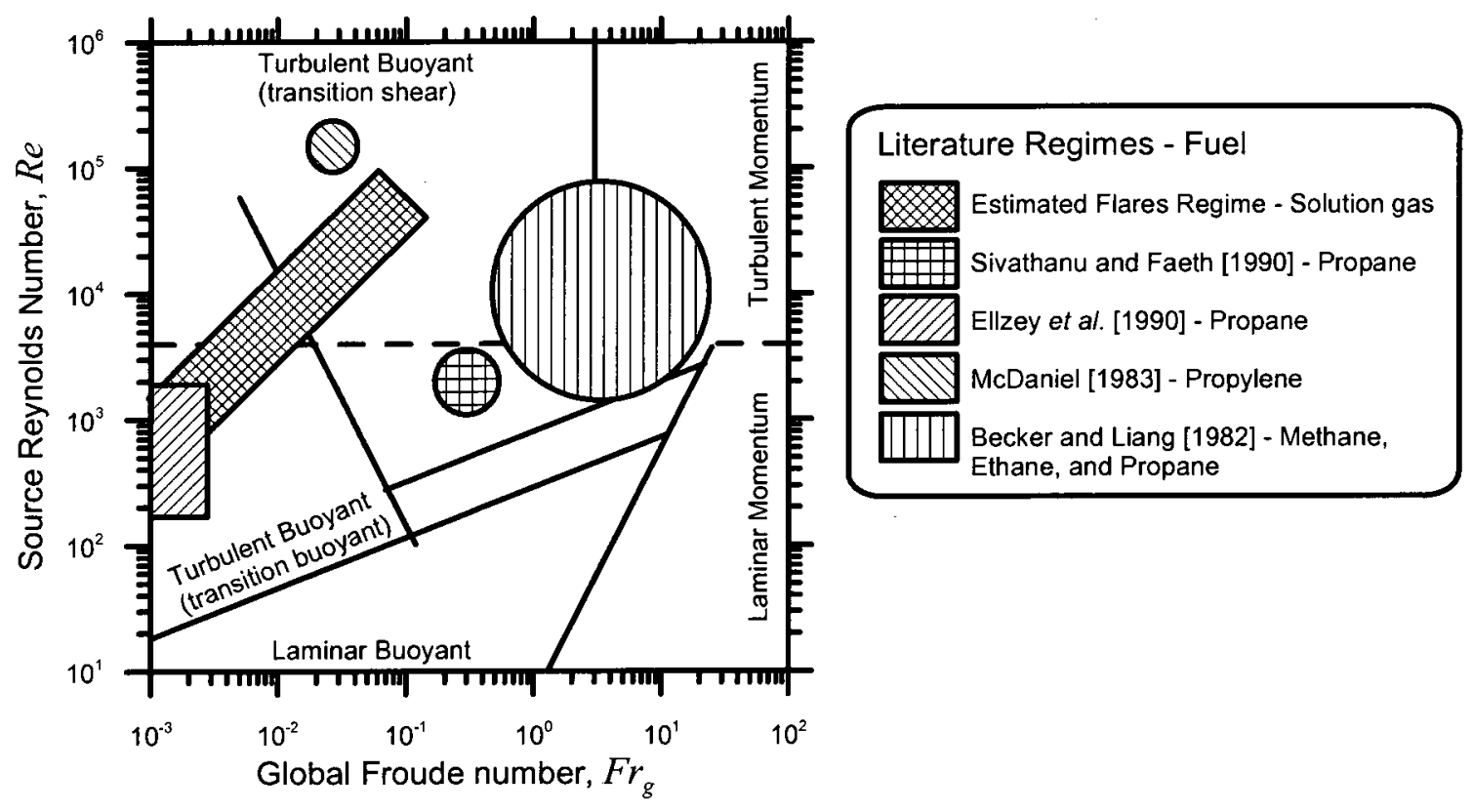

Figure 2.6 - Comparison of flare regime with estimated regimes of available literature. 


\section{Chapter 3}

\section{Experimental Setup}

The purpose of the LSF project is to generate flames relevant to those of solution gas flares in the UOG industry and measure the total soot emission at zero crosswind conditions. The experimental setup described below was originally commissioned by Canteenwalla [2007], and is located at NRC facilities.

A modular burner and fuel delivery system were employed to permit a wide range of operating conditions. Fuel mixtures consisting of any or all of $\mathrm{CH}_{4}, \mathrm{C}_{2} \mathrm{H}_{6}, \mathrm{C}_{3} \mathrm{H}_{8}$, $\mathrm{C}_{4} \mathrm{H}_{10}, \mathrm{CO}_{2}$, and $\mathrm{N}_{2}$ could be metered, mixed, and delivered to the burner as shown in Figure 3.1. All of the combustion products from the LSF were collected using an enclosure and dilution tunnel. Emission sampling was done from the dilution tunnel (DT) and samples were collected on filters or measured by an LII system and used to calculate the total soot emission rates.

Compared to the original apparatus used by Canteenwalla [2007], several major changes to the setup were made to support the research presented in this thesis. Most notably, these included modifications to the fuel delivery system, increased dilution capabilities, an improved DT flow measurement technique, and a new gravimetric 
sampling system. An additional burner tip with an inner diameter of $76.2 \mathrm{~mm}$ was added to extend the capabilities of the experimental setup from the previous maximum of $50.8 \mathrm{~mm}$.
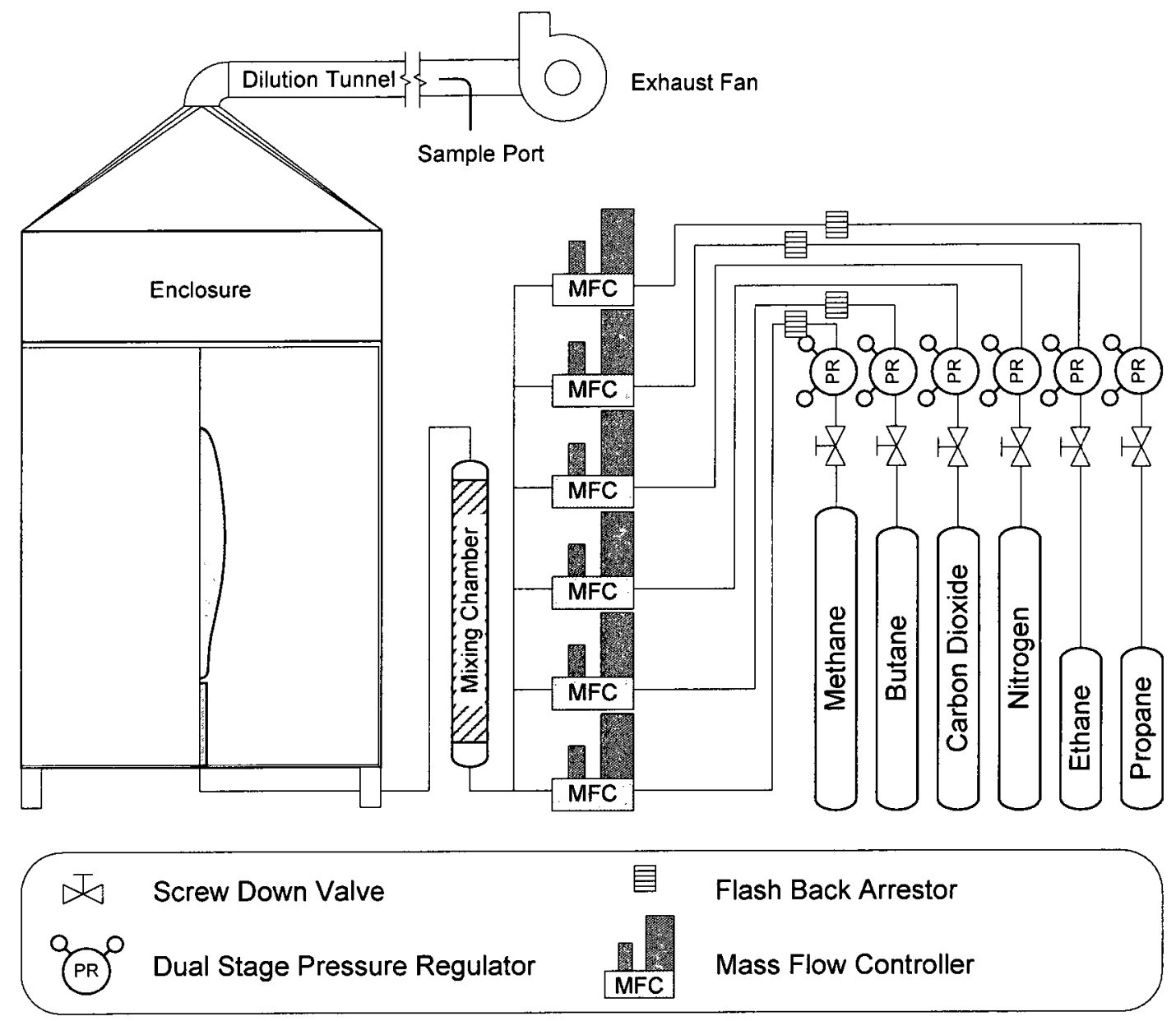

Figure 3.1 - Schematic of Lab Scale Flare (LSF) fuel delivery system, enclosure, and dilution tunnel.

\subsection{Turbulent Diffusion Burner}

The modular burner originally designed by Canteenwalla [2007] was intended to allow for burner tip sizes of up to $76.2 \mathrm{~mm}$ (although only sizes up to $50.8 \mathrm{~mm}$ were used in his work). The burner was composed of five main components: the diffuser, settling chamber, converging nozzle, turbulence generating grids, and burner exit tube as shown in Figure 3.2. 


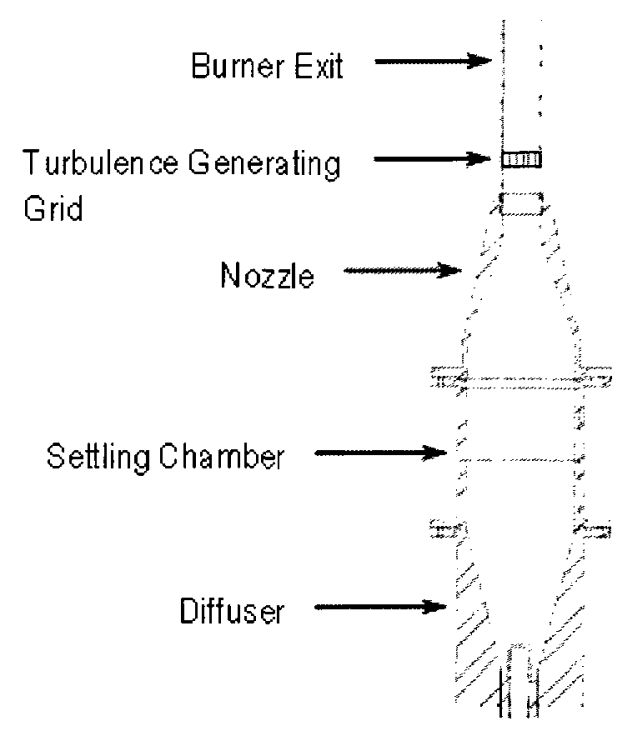

Figure 3.2 - Schematic of the LSF [Canteenwalla, 2007].

In the current work five different burners, with inner diameters of 0.5 " $(12.7 \mathrm{~mm})$, 1" $(25.4 \mathrm{~mm}), 1.5 "(38.1 \mathrm{~mm}), 2 "(50.8 \mathrm{~mm})$, and 3" $(76.2 \mathrm{~mm})$, were used. The outer diameter of the burner tips were machined to match the ratio of the inner to the outer diameter of a standard 4" NPS pipe used in a "typical" flare, which was 0.89 . The exit lengths of the burners are listed in Table 3.1. The turbulence generating grids were placed such that the velocity profiles at the exit plane mimicked those of fully developed turbulent pipe flow, and so that the turbulence intensity in the core of the flow was approximately $3-6 \%$ for all burners. For details of the turbulence intensity measurements and calculations, see Appendix A from Canteenwalla [2007].

Table 3.1 - Burner Tip Dimensions

\begin{tabular}{|c|c|c|}
\hline $\begin{array}{c}\text { Burner Inner } \\
\text { Diameter (mm) }\end{array}$ & $\begin{array}{c}\text { Total Burner Exit } \\
\text { Length (mm) }\end{array}$ & $\begin{array}{c}\text { Length from Turbulence } \\
\text { Grid to Burner Exit (mm) }\end{array}$ \\
\hline 12.7 & 133 & 127 \\
\hline 25.4 & 133 & 127 \\
\hline 38.1 & 133 & 77 \\
\hline 50.8 & 266.7 & 254 \\
\hline 76.2 & 381 & 375 \\
\hline
\end{tabular}




\subsection{Fuel Delivery System}

The fuel delivery system includes six separate Brooks mass flow controllers (Smart Series models $5850 \mathrm{~S} / \mathrm{BC}(3), 5851 \mathrm{~S} / \mathrm{BD}(1), 5853 \mathrm{~S} / \mathrm{BE}(1), 5850 \mathrm{~S} / \mathrm{BA}(1))$ that control the flows of the individual component gases supplied in pressurized cylinders. Using these controllers, it was possible to generate fuel mixtures of up to 6-components comprising methane, ethane, propane, butane, carbon dioxide, and nitrogen. Total flow rates of the mixtures ranging from 5 to 110 standard litres per minute (SLPM, where standard is defined as $101325 \mathrm{~Pa}$ barometric pressure and $273 \mathrm{~K}$ temperature) could be achieved. Each mass flow controller was calibrated following one of two procedures, depending on the maximum flow rate of that controller, as outlined in Appendix B. These calibrations allowed accurate operation over the full scale of the controller with an uncertainty of approximately $\pm 1-3 \%$, depending on the controller calibration ${ }^{1}$. Downstream of the flow controllers the fuel components were thoroughly mixed in a $1 \mathrm{~m}$ long, $50.8 \mathrm{~mm}$ diameter, vertical mixing chamber filled with glass beads.

\subsubsection{Fuel Mixture Compositions}

Up to six separate components of the fuel mixture (four hydrocarbons and two inerts) were chosen based on the analysis of data from solution gas samples at 2908 oil production sites in Alberta. These data were received as a private communication through the technical steering committee of PTAC, specifically to support this work [private communication between Dr. Matthew Johnson and PTAC, 2007]. Analysis of these data has shown that methane is the major constituent of the gases being flared.

\footnotetext{
${ }^{1}$ Calibrations are given in Appendix B.
} 
Table 3.2 shows the average concentrations by volume of all gases sampled and Figure 3.3 plots these same data along with $10^{\text {th }}$ and $90^{\text {th }}$ percentiles of species concentration.

Table 3.2 - Average well composition from 2908 sites in Alberta. C1-C7 represents the number of carbon atoms in the hydrocarbon compound.

\begin{tabular}{|c|c|}
\hline Species & Average Composition (\%) \\
\hline $\mathrm{C} 1$ & 83.395 \\
\hline $\mathrm{C} 2$ & 6.902 \\
\hline $\mathrm{C} 3$ & 3.045 \\
\hline $\mathrm{C} 4$ & 1.412 \\
\hline $\mathrm{C} 5$ & 0.503 \\
\hline $\mathrm{C} 6$ & 0.204 \\
\hline $\mathrm{C} 7$ & 0.339 \\
\hline $\mathrm{H}_{2} \mathrm{~S}$ & 1.075 \\
\hline $\mathrm{CO}_{2}$ & 1.863 \\
\hline $\mathrm{N}_{2}$ & 1.217 \\
\hline $\mathrm{He}_{2}$ & 0.029 \\
\hline $\mathrm{H}_{2}$ & 0.018 \\
\hline
\end{tabular}

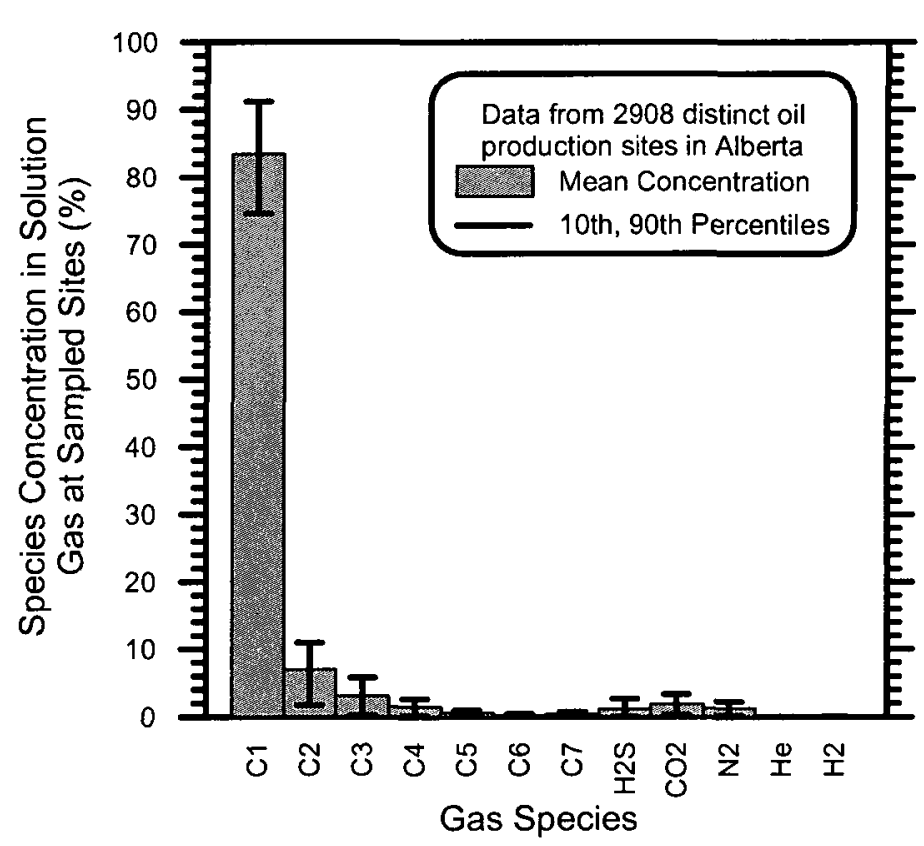

Figure 3.3 - Mean, $10^{\text {th }}$, and $90^{\text {th }}$ Percentile Solution Gas Concentrations [private communication between Dr. Matthew Johnson and PTAC, 2007]

To reduce the complexity of the experiment, surrogate mixtures were used based on the six most abundant components (i.e. mixtures of $\mathrm{C} 1-\mathrm{C} 4, \mathrm{CO} 2$, and $\mathrm{N} 2$ ). Higher hydrocarbons $\mathrm{C} 5$ through $\mathrm{C} 7, \mathrm{He}$, and $\mathrm{H}_{2}$ were not included in the test mixtures due to their low concentrations. Hydrogen sulphide $\left(\mathrm{H}_{2} \mathrm{~S}\right)$ was also neglected primarily due to 
its extreme toxicity ${ }^{1}$, but also because it was absent from most fuel mixtures (a.k.a. sweet gas). While the average fraction of $\mathrm{H}_{2} \mathrm{~S}$ in the sampled flare gas was also quite low at $1.075 \%$, the $75^{\text {th }}$ percentile concentration was even lower at $0.23 \%$. Thus, $\mathrm{H}_{2} \mathrm{~S}$ only affected a small portion of the sites tested, and had very low concentrations at the majority of those sites.

Although detailed speciation of hydrocarbon components was not available in the provided data, typical components in natural gas from both dry and wet reservoirs are alkane-based [Smith et al., 1992]. Furthermore, results from a limited sampling of six operating flares in Alberta have also confirmed that the principle hydrocarbons were all alkane-based [Kostiuk et al., 2004]. Thus, in the present work the hydrocarbon fuel species $\mathrm{Cl}$ through $\mathrm{C} 4$ were assumed to be alkanes.

In the case of the C4 fuel component, butane, two isomers exist: iso-butane and $n$ butane. In the present experiments $\mathrm{C} 4$ was assumed to be exclusively $n$-butane, since it accounted for approximately $\sim 65 \%$ of the total butane volume fraction in the provided samples, and $n$-butane was economical to use for these tests. The work of Schalla and McDonald [1953] is one of the few studies to measure sooting tendencies of both $n$ - and iso-butane with the same experimental setup. While the two fuels have very different smoke-point flow rates (Table 3.3), their TSI values, which should be a better measure of sooting tendency and which were calculated using the same data of Schalla and McDonald [1953], suggest more comparable performance. If a mixture of $65 \% n$-butane

\footnotetext{
${ }^{1}$ The Material Safety Data Sheet (MSDS) [AirLiquide, 2008] for $\mathrm{H}_{2} \mathrm{~S}$ classifies it as a toxic, flammable, colorless, liquefied gas. There are many adverse health effects from low and high concentration exposures including inflammation and irritation of the eyes at exposure concentrations of less than $10 \mathrm{ppm}$. Above 50 $\mathrm{ppm}$, there is an intense tearing, blurring of vision, and pain when looking at light. Exposures can be fatal as well, if exposed to the gas for more than 30 minutes at concentrations of $600 \mathrm{ppm}$ or greater.
} 
and $35 \%$ iso-butane were used, ideal gas mixing laws estimate that the sooting tendency would only increase by $\sim 15 \%$. This represents a small increase when the amount of butane ( $1.412 \%$ by volume) in the average PTAC mixture is considered, and the effects of neglecting iso-butane in the mixture were deemed negligible.

Table 3.3 - Comparison of sooting tendencies of $\boldsymbol{n}$ - and iso-butane

\begin{tabular}{|c|c|c|c|}
\hline & n-Butane & iso-Butane & $\begin{array}{c}\text { 65\% } \mathbf{n - / 3 5 \%} \text { iso- } \\
\text { Butane Mixture }\end{array}$ \\
\hline $\begin{array}{c}\text { Smoke-point [Schalla and McDonald, 1953] } \\
\text { (mg/s) }\end{array}$ & 8.60 & 4.01 & 7.00 \\
\hline $\begin{array}{c}\text { TSI [Calcote and Manos, 1983] based on } \\
\text { smoke-points of Schalla and McDonald [1953] }\end{array}$ & 1.6 & 2.2 & 1.81 \\
\hline $\begin{array}{c}\text { TSI [Olson } \text { et al., 1985] based on smoke-points } \\
\text { of Schalla and McDonald [1953] }\end{array}$ & 2.0 & 2.8 & 2.28 \\
\hline
\end{tabular}

Surrogate test mixtures of flare gas were created by scaling selected component concentrations by their concentrations in the full mixture as listed in Table 3.4. Lighter and heavier fuel mixtures were also created (Table 3.4) to investigate the effects of fuel composition on soot yield. To create the light mixture, the $90^{\text {th }}$ percentile methane concentration was chosen as an upper bound and the remaining fuel concentrations were determined based on their relative concentrations in the average fuel mixture (i.e. propane to ethane ratio constant, etc.). The heavy mixture was created in the same manner except that the $10^{\text {th }}$ percentile methane concentration was chosen as a lower bound. The same process was repeated neglecting the diluents of $\mathrm{CO}_{2}$ and $\mathrm{N}_{2}$ to create pure fuel mixtures. The concentrations of all surrogate gas mixtures used in this study are summarized in Table 3.4 and illustrated in Figure 3.4. 
Table 3.4 - Average, Heavy, and Light fuel mixtures

\begin{tabular}{|c|c|c|c|c|c|c|c|}
\hline \multirow{2}{*}{ Species } & \multirow{2}{*}{ Purity (\%) } & \multicolumn{2}{|c|}{ Average Mixture (\%) } & \multicolumn{2}{c|}{ Light Mixture (\%) } & \multicolumn{2}{c|}{ Heavy Mixture (\%) } \\
\cline { 3 - 8 } & & 6-Mix & 4-Mix & 6-Mix & 4-Mix & 6-Mix & 4-Mix \\
\hline Methane & 99.0 & 85.24 & 88.01 & 91.14 & 91.14 & 74.54 & 74.54 \\
\hline Ethane & 99.0 & 7.06 & 7.28 & 4.23 & 5.38 & 12.17 & 15.47 \\
\hline Propane & 99.0 & 3.11 & 3.21 & 1.87 & 2.38 & 5.37 & 6.83 \\
\hline n-Butane & 99.0 & 1.44 & 1.5 & 0.87 & 1.1 & 2.49 & 3.16 \\
\hline $\mathrm{CO}_{2}$ & 99.99 & 1.91 & 0 & 1.14 & 0 & 3.28 & 0 \\
\hline $\mathrm{N}_{2}$ & 99.999 & 1.24 & 0 & 0.75 & 0 & 2.15 & 0 \\
\hline
\end{tabular}

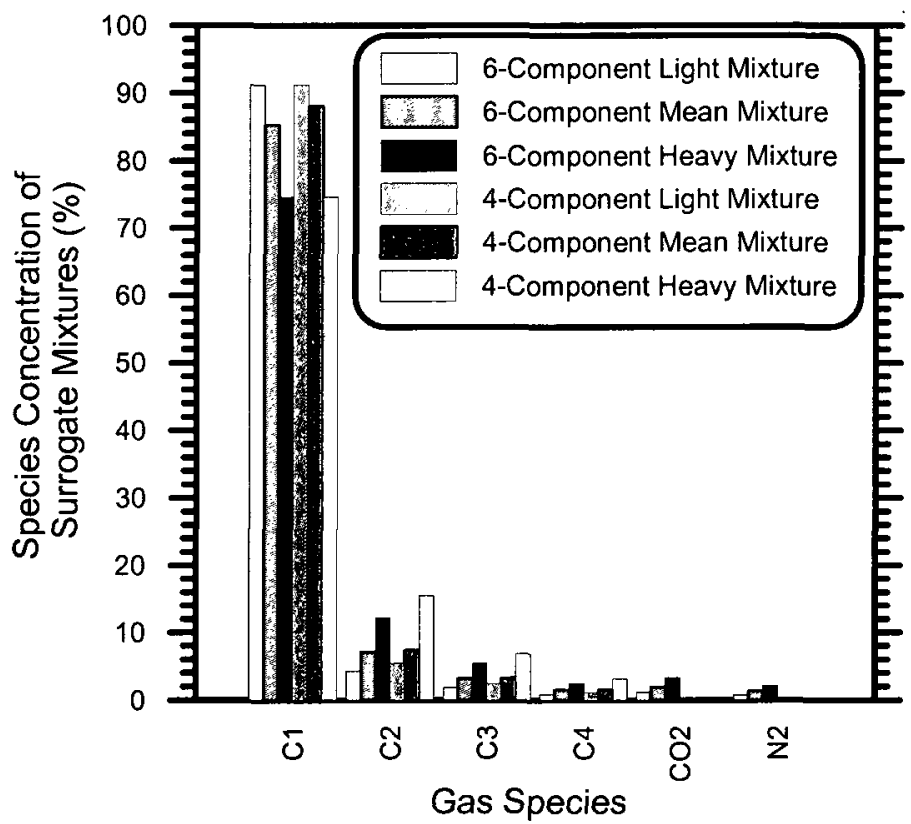

Figure 3.4 - Light, Average, and Heavy 6-mix surrogate fuel mixtures

\subsubsection{Enclosure and Emission Collection System}

The LSF enclosure remained relatively unchanged from the work of Canteenwalla [2007], which was designed based on the work of Sivathanu and Faeth [1990]. The LSF burner was centered inside the $1.5 \times 1.5$ by $2.6 \mathrm{~m}$ tall sheet metal enclosure that sat $50 \mathrm{~mm}$ above the floor. The front of the enclosure had two doors, one made of steel sheet metal and the other of Plexiglas to provide visual access to the LSF. The top of the enclosure contracted and directed the entire exhaust plume and entrained dilution air into a $152.4 \mathrm{~mm}$ I.D. insulated, galvanized steel pipe. The $152.4 \mathrm{~mm}$ pipe acted as a dilution tunnel where the combustion products and room air were mixed and provided a steady 
medium from which to extract emission samples as shown in Figure 3.5. The flow of diluted exhaust was assisted by an industrial centrifugal fan capable of drawing 13,000 LPM through the DT and controlled using a variable speed motor and controller. The fan was a Cincinnati Fan cast aluminum pressure blower (model PB-14A) with a 6" inlet and $12 \frac{1 / 4}{4}$ diameter fan wheel. The fan was mounted on the shaft of a WEG general-purpose three-phase, $3 \mathrm{HP}$ motor in a NEMA 56 frame (F $56 \mathrm{H})$ with a rated speed of $3450 \mathrm{rpm}$. The controller was a Leeson SM series variable frequency drive that was controlled by a computer via a variable current (4-20 mA) input.

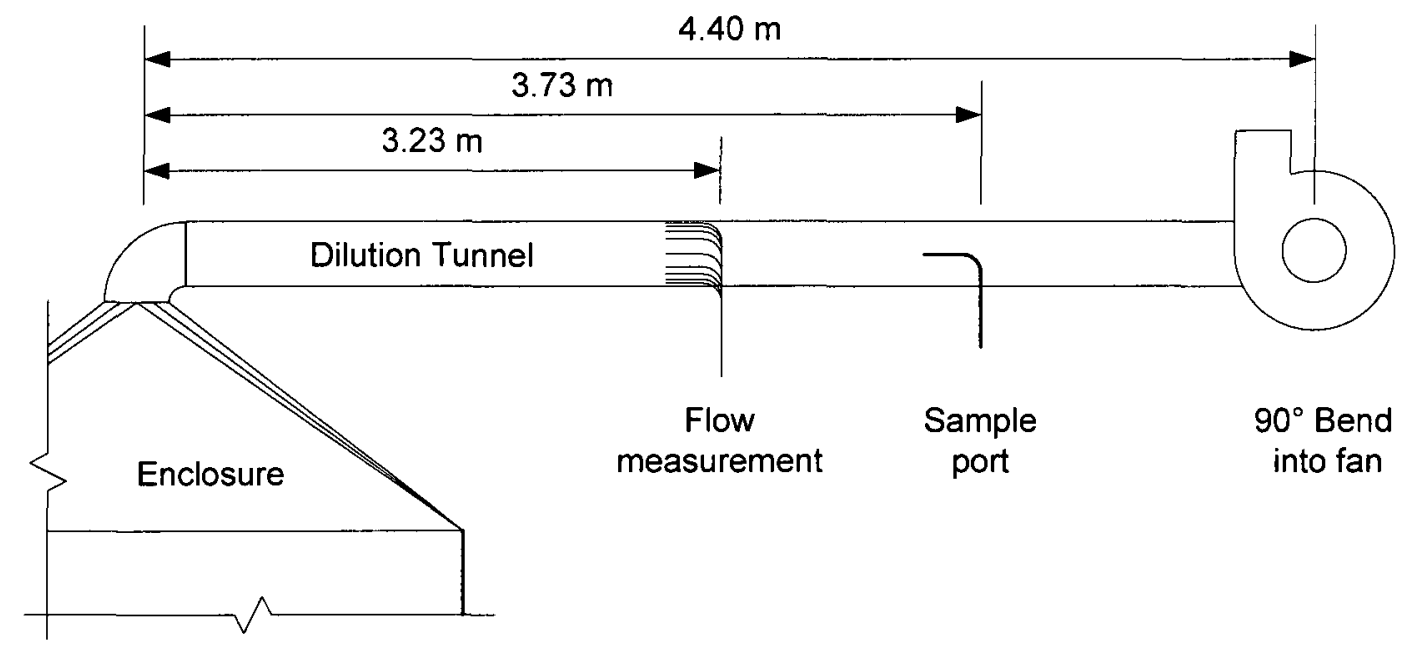

Figure 3.5 - Diagram of dilution tunnel and components

In designing his experiment, Canteenwalla [2007] followed literature [Chang et al., 2004] that suggested any dilution ratio (defined according to Equation 3.1) above 20 should produce consistent results, but his results showed that this was not the case. Instead, he observed that the total soot emission as measured by the LII would change under different dilution ratios. This effect could not be isolated or corrected, and followed no noticeable trends. Prior to commencing the present work, it was decided to revisit the determination of an appropriate dilution ratio for soot sampling. 


$$
D R=\frac{Q_{\text {DilltionAir }}}{Q_{\text {Products }}}
$$

Several pertinent measurement protocols for soot sampling of stationary sources were reviewed to identify any critical parameters that may help control the dilution ratio effects. These included the USEPA [2005] Engine Testing Procedures (ETP), the USEPA [2000] Emissions Measurement Centre (EMC) Method 5 - Particulate Matter from stationary sources, and the UNECE [2008] Vehicle Regulation No. 49. These three protocols were analyzed to ensure that the current DT arrangement did not violate any recommendations from these protocols. Although no specific guidelines existed for sampling from lab-scale flares, the consulted protocols were useful because they suggest general equipment and arrangements for the size of the DT, the DT flow rate measurement method, the sample probe type, the distance from the flow measurement and sample probe to the nearest disturbance, minimum DT flow rates, and conditions from the sample probe to the sampling device. The system developed for the LSF met or exceeded most standards. Table 3.5 compares the experimental setups as stipulated under the different standard sampling methods as well as the updated conditions used in the present setup.

Most minimum requirements were met, except for the diameter of the dilution tunnel as specified in the UNECE regulation [2008], the iso-kinetic sampling condition, and the temperature upstream of the filter from USEPA ETP [2005]. The UNECE regulation is for heavy vehicles where the exhaust is expected to be extremely hot initially, and to contain very high concentrations of soot. 
Table 3.5 - Comparison of sampling protocols

\begin{tabular}{|c|c|c|c|c|}
\hline & Current Setup & USEPA ETP [2005] & $\begin{array}{c}\text { UNECE Reg. } \\
\mathbf{4 9}[\mathbf{2 0 0 8}]\end{array}$ & $\begin{array}{c}\text { USEPA EMC } \\
{[2000]}\end{array}$ \\
\hline $\begin{array}{c}\text { Intended } \\
\text { application }\end{array}$ & Lab-scale flares & PM from engines & $\begin{array}{c}\text { PM from } \\
\text { heavy vehicles }\end{array}$ & $\begin{array}{c}\text { PM from } \\
\text { stationary sources }\end{array}$ \\
\hline DT diameter & $6 "$ & Not specified & $>8^{\prime \prime}$ & $4-12^{\prime \prime}$ \\
\hline $\begin{array}{c}\text { Flow } \\
\text { measurement }\end{array}$ & $\begin{array}{c}\text { Pitot rake, 17 } \\
\text { measurements }\end{array}$ & $\begin{array}{c}\text { Critical flow venturi, } \\
\text { PDP, subsonic venturi, } \\
\text { ultrasonic flow meter }\end{array}$ & $\begin{array}{c}\text { Critical flow } \\
\text { venturi, PDP }\end{array}$ & $\begin{array}{c}\text { Standard Pitot } \\
\text { tube }\end{array}$ \\
\hline Sample inlet & $\begin{array}{c}\text { Pitot tube, 1/4", } \\
\text { upstream facing }\end{array}$ & Upstream facing probe & $\begin{array}{c}\text { Upstream } \\
\text { facing probe }\end{array}$ & $\begin{array}{c}\text { Elbow or button } \\
\text { hook }\left(1 / 8-1 / 2^{\prime \prime}\right)\end{array}$ \\
\hline $\begin{array}{c}\text { Elbow to flow } \\
\text { measurement }\end{array}$ & 21.2 diameters & $>10$ diameters & Not specified & $>16$ diameters \\
\hline $\begin{array}{c}\text { Elbow to } \\
\text { sample inlet }\end{array}$ & 24.47 diameters & Not specified & $>10$ diameters & $>8$ diameters \\
\hline $\begin{array}{c}\text { DT flow rates } \\
\text { Min Re of } \\
10,000\end{array}$ & Min Re of 4,000 & $\begin{array}{c}\text { Min } R e \text { of } \\
4,000\end{array}$ & Not specified \\
\hline $\begin{array}{c}\text { Temperature } \\
\text { upstream of } \\
\text { filter }\end{array}$ & $\begin{array}{c}<52^{\circ} \mathrm{C} \\
\text { (maintained } \\
\left.\text { within } \pm 5^{\circ} \mathrm{C}\right)\end{array}$ & $\begin{array}{c}47 \pm 5^{\circ} \mathrm{C} \\
\text { (maintained } \\
\left.\text { within } \pm 3^{\circ} \mathrm{C}\right)\end{array}$ & $120 \pm 14^{\circ} \mathrm{C}$ \\
\hline $\begin{array}{c}\text { Iso-kinetic } \\
\text { conditions? }\end{array}$ & No & Approximate & Yes & Within $10 \%$ \\
\hline $\begin{array}{c}\text { Sample device } \\
\text { distance from } \\
\text { sample port }\end{array}$ & $\begin{array}{c}0.5 \mathrm{~m}, \text { insulated } \\
\text { insulated }\end{array}$ & $\begin{array}{c}\text { As close as possible, } \\
<1.0 \mathrm{~m}\end{array}$ & Immediate \\
\hline
\end{tabular}

It was decided that the most important aspect of the DT was that the exhaust gases be fully mixed to ensure that the extracted sample was representative of the average concentration and that the exact diameter was less important. The fully mixed requirement was satisfied by ensuring that the Reynolds number was higher than 4000 , and that the sample inlet was sufficiently far downstream of any disturbances to ensure that the exhaust gases were completely mixed. To ensure that the exhaust gases were indeed well mixed, a traverse of the duct was completed to measure the soot volume fraction profile using the LII device (see section 3.3.2 for LII details). This test was completed both at the minimum and maximum expected DT flow rates. The results of these tests, summarized in Figure 3.6 (a), showed that for the limiting flow rate condition, the exhaust in the dilution tunnel was well mixed. Similar results for the maximum flow rate condition are shown in Figure 3.6 (b). To ascertain the importance of iso-kinetic 
sampling, tests were completed with the sample probe facing downstream. The results of these tests are included in Figure 3.6, and showed no discernable difference from the measurements performed with the probe facing upstream. This led to the conclusion that iso-kinetic sampling was not a critical parameter in the sampling protocol.

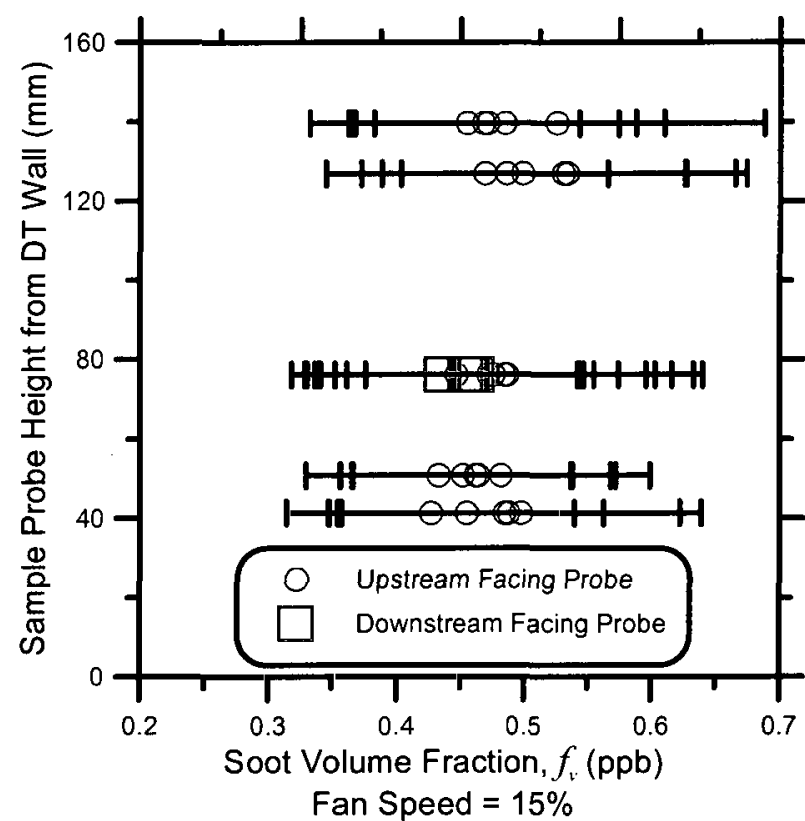

(a)

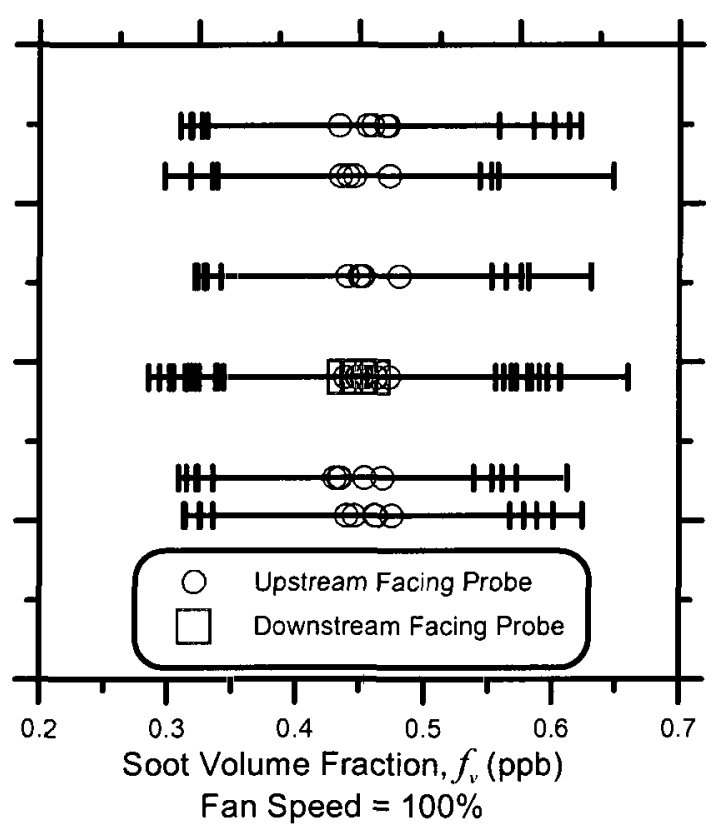

(b)

Figure 3.6 - Soot concentration traverse at fan speeds of (a) $15 \%$ and (b) $100 \%$. Error bars represent standard deviations of LII measured $\boldsymbol{f}_{v}$

When comparing sampling conditions between the cited sampling protocols, it became evident that all protocols controlled the temperature upstream of the sampling device (typically a filter assembly). For the present work, temperature was directly related to the dilution ratio in the DT. More dilution air lowered the DT temperature and increased the dilution ratio. Thus, the temperature upstream of the measurement device was monitored and controlled instead of directly monitoring and controlling the dilution ratio. The three referenced protocols all suggested a different upstream temperature, so the median value of $52 \pm 5^{\circ} \mathrm{C}$ was chosen for the current protocol. 


\subsubsection{Measurement of Sample Duct Flow Rate}

A major departure from the previous experimental configuration of Canteenwalla [2007] was in the measurement of the flow rate in the DT. In the work of Canteenwalla [2007], an averaging Pitot tube was used to find the total flow rate. However, it was determined that this led to an unknown uncertainty in the flow rate measurement because the averaging Pitot tube assumed an axi-symmetric velocity profile which vertical and horizontal Pitot probe traverses showed not to be the case.

For the current work, the flow rate was measured using a permanently installed Pitot probe rake. The rake consists of 17 Pitot probes that were positioned to measure representative velocity profiles in the horizontal and vertical directions as shown in Figure 3.7. The single port probes only measured the total pressure and so it was necessary to measure the static pressure separately using four static ports that were cut into to the DT at $90^{\circ}$ intervals on the same plane as the inlet to the Pitot probes. The static ports were offset from the probe's angular orientation by $45^{\circ}$ to avoid any potential impacts from the Pitot probe closest to the wall, also shown in Figure 3.7. The four static taps were connected into one manifold, and the averaged static pressure was measured and used for calculations of density in the DT and as a reference for differential pressure measurements.

The Pitot probes were constructed of $3.175 \mathrm{~mm}$ O.D. stainless steel tubing with an I.D. of $1.588 \mathrm{~mm}$. A custom bending tool was created to ensure that each tube was bent to $90^{\circ}$ within $\pm 0.5^{\circ}$. The minimum length of the probe neck was determined based on the empirical work of Prandtl as summarized by Goldstein [1996]. This work suggests that the probe tip should be no less than 10 pipe diameters ahead of the stem, and in the 
current setup, the minimum distance is 24 pipe diameters. Attached directly to each Pitot probe was a bare wire thermocouple located 10 pipe diameters downstream $(31.75 \mathrm{~mm})$ from the tip of the probe.

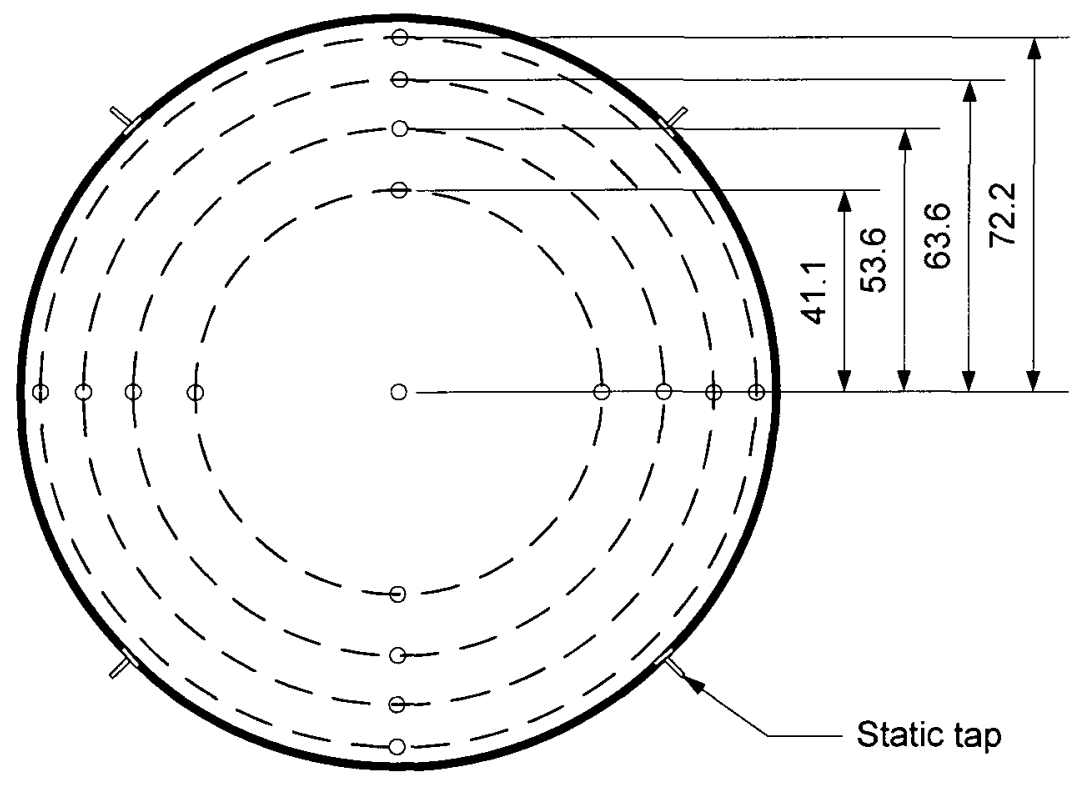

Figure 3.7 - Diagram of Pitot probes and static ports. Radial probe locations in mm

The static taps were securely fastened and sealed to the outside of the DT. The inside bore of the taps were then drilled out through the DT wall, so that the tap had a constant diameter through to the exit. The tap dimensions were designed according to Prandtl as summarized by Goldstein [1996]. Each tap hole was filed flat on the inside of the DT to ensure that there were no countersinking or rounding effects at the taps.

The differential pressure was measured at each of the seventeen Pitot tubes by an individual variable capacitance pressure transducer (Ashcroft XLdp, IXLdp). Depending on the location of the Pitot probe within the duct, an appropriately ranged pressure transducer was used (from 0.5 to 2.5 inches of $\mathrm{H}_{2} \mathrm{O}$ full-scale). The local density was calculated at each Pitot probe based on the average static pressure and the temperature measured at the probe. Using the differential pressure, or dynamic pressure, and the gas 
density determined using the measured temperature along with the ideal gas assumption, the velocity at the Pitot was calculated using Equation 3.2.

$$
u=\sqrt{\frac{2 P_{d}}{\rho}}
$$

The seventeen individual velocity measurements yielded four radial velocity profiles in the horizontal and vertical directions. Assuming zero velocity at the DT wall, the velocities were combined using an uneven trapezoidal numerical integration from $r=0$ at the centre to $r=76.2 \mathrm{~mm}$ at the wall to find the flow rate. Equation 3.3 is the general equation for an unevenly spaced trapezoidal numerical integration. The real integral is shown in Equation 3.4. The general form of numerical integration was modified using an area substitution for the volume flow rate expression as shown in Equation 3.5. Each profile was integrated assuming that the profile was constant $\pm 45^{\circ}$ from each line of integration, $j=1$ to 4 :

$$
\begin{gathered}
I=\int x d t \cong \sum_{i=0}^{n-1} \frac{\left(x_{i}+x_{i+1}\right)}{2}\left(t_{i+1}-t_{i}\right) \\
Q_{d, j}=\int u(r) \frac{1}{4} d A=\int u(r) \frac{\pi}{4} r d r \\
\therefore Q_{d, j} \cong \frac{\pi}{4} \sum_{i=0}^{n-1} \frac{\left(u_{i}+u_{i+1}\right)}{2}\left[\left(r_{i+1}\right)^{2}-\left(r_{i}\right)^{2}\right]
\end{gathered}
$$

where $u$ is the discrete velocity data, $r$ is the radial location, and $n$ is the number of discrete data points, which for all four cases is six $\left(u_{l}\right.$ at $r=0, u_{2}$ at $r=41.1 \mathrm{~mm}, u_{3}$ at $r=53.6 \mathrm{~mm}, u_{4}$ at $r=63.6 \mathrm{~mm}, u_{5}$ at $r=72.2 \mathrm{~mm}$, and $u_{6}=0$ at $r=76.2 \mathrm{~mm}$ ). These four partial flow rates were summed to give the total flow rate through the dilution tunnel. A similar integration process was completed using the Simpson's $1 / 3$ rule, which resulted in a DT flow rate that was lower by $\sim 0.55 \%$, a negligible amount. An example 
of the four radial profiles (two in the horizontal and vertical directions) are shown in Figure 3.8.

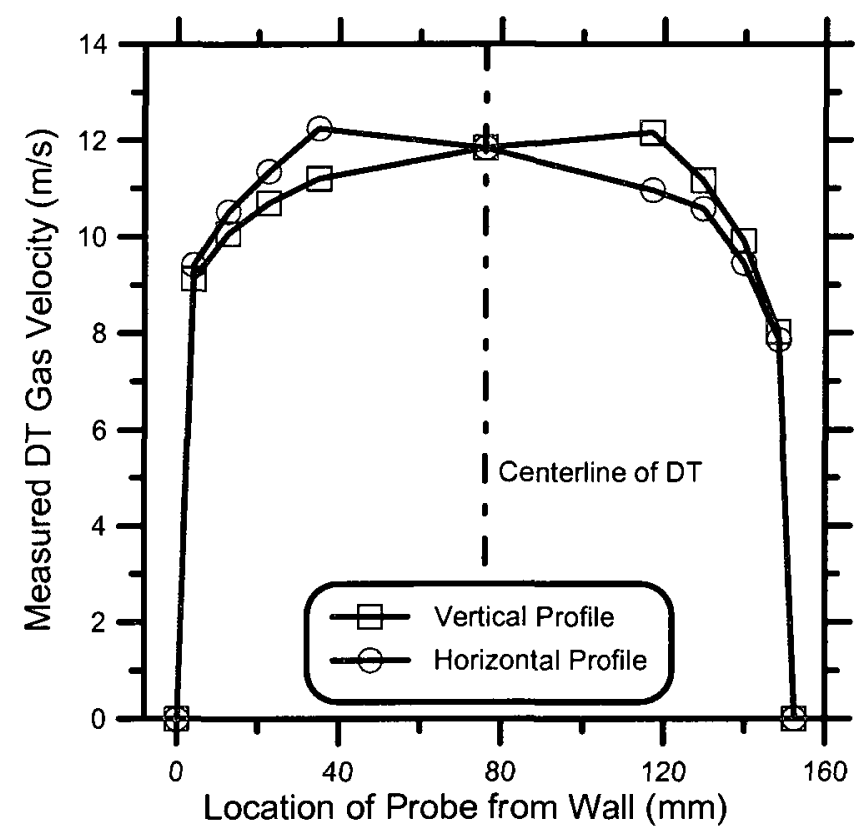

Figure 3.8 - Velocity profiles in the horizontal and vertical directions at a fan speed of $100 \%$

\subsection{Soot Sampling System}

Two different techniques were used to measure the soot volume fraction in the dilution tunnel. Measurements were initially completed using a new gravimetric analysis system and sampling protocol, and additional data were collected using an LII method. The work of Canteenwalla [2007] originally used an LII instrument for all soot volume fraction measurements, but it was believed that the chosen method might have been introducing considerable uncertainty in the results. Subsequent to his work, defects were found with the instrument he used, confirming this suspicion. It was therefore decided to change the primary measurement method to the gravimetric system, where filters deposited with soot were measured pre- and post-test to determine the amount of soot 
collected. The gravimetric-based measurements would also provide a baseline for comparing LII-based measurements.

\subsubsection{Gravimetric Analysis}

Gravimetric analysis is a relatively straightforward technique in which the mass of soot $\left(m_{s o o t}\right)$ collected on a filter is determined by measuring the difference in filter weight before and after sampling. The volume of exhaust gas drawn through the filter, $V_{\text {sample }}$, is measured, and when combined with an assumed soot density, $\rho_{\text {soot }}$, from literature, the soot volume fraction can be determined. The soot volume fraction was then simply the ratio of the sampled soot volume to the sampled exhaust gas volume, as shown in Equation 3.6.

$$
f_{v}=\frac{m_{\text {soot }}}{\rho_{\text {soot }} V_{\text {sample }}}
$$

\subsubsection{Soot Sampling Train}

The sampling train was composed of five main components as shown in Figure 3.9. These were: a sample probe, a secondary dilution system, a filter holder, a total flow measurement device, and a vacuum pump. This sampling arrangement was modelled after generic sampling systems used to measure PM in diesel engine exhaust, which typically employ some combination of a primary DT as discussed previously and secondary dilution if required in their filter sampling setup [USEPA, 2005].

The sample probe was a $6.35 \mathrm{~mm}$ I.D., $9.50 \mathrm{~mm}$ O.D. stainless steel tube bent into the shape of a Pitot probe. The neck of the probe was $110 \mathrm{~mm}$ long, with the remaining section of pipe extending $76.2 \mathrm{~mm}$ to the DT wall, and a further $200 \mathrm{~mm}$ outside the 
insulated DT. This extension of the probe beyond the exterior of the DT wall was insulated with $25 \mathrm{~mm}$ thick fibreglass insulation.
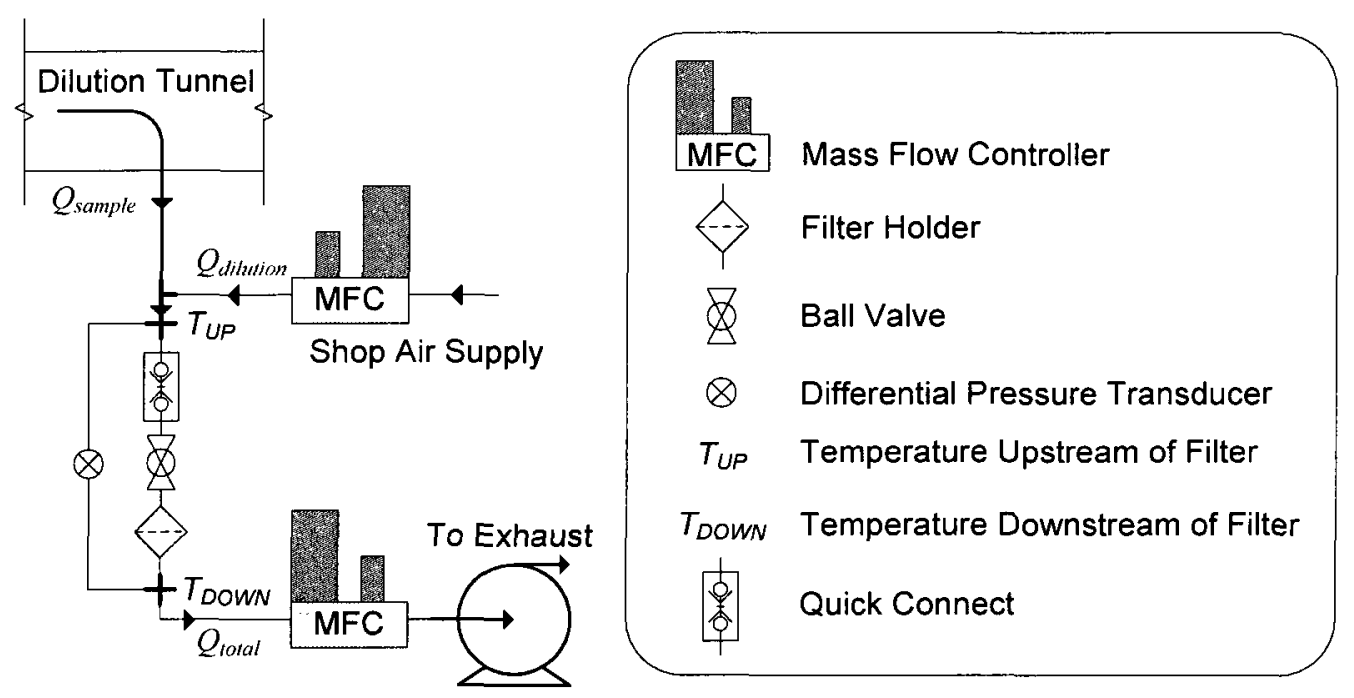

Figure 3.9 - Schematic of Gravimetric sampling train.

The secondary dilution system was attached downstream of the probe and was simply a controlled, room temperature air supply used to cool the raw exhaust gases to the required temperature upstream of the filter, i.e. $<52^{\circ} \mathrm{C}$. The secondary dilution air was introduced through a t-joint. After a short section of piping $(6.35 \mathrm{~mm}$ I.D., $75 \mathrm{~mm}$ long) the temperature of the gas was measured using a standard K-type thermocouple.

A full flow quick-connect fitting was located downstream of the temperature measurement $(65 \mathrm{~mm})$ to allow easy switching between selected measurement methods (gravimetric or LII). A ball valve was placed downstream of the quick-connect $(100 \mathrm{~mm})$ to seal off the filter holder between tests and prevent contamination of the filters. The filter holder was mounted immediately downstream of the ball valve. A custom-designed and manufactured aluminum filter holder was developed to ensure an even distribution of soot particles on the filter and to ensure that the filter holder did not induce a significant pressure drop in the sampling train (many of the filter holders 
available for purchase had very sharp expansions and contractions). As discussed in section 3.3.1.2, $47 \mathrm{~mm}$ filters were used. The main purpose of the filter holder was to: 1) support the filter, 2) seal the filter into the flow stream, 3) minimize losses in the system, and 4) allow easy removal of filters without sample loss, contamination, or destruction. A schematic of the filter holder is shown in Figure 3.10 along with a photograph of the filter assembled as installed in the current setup.

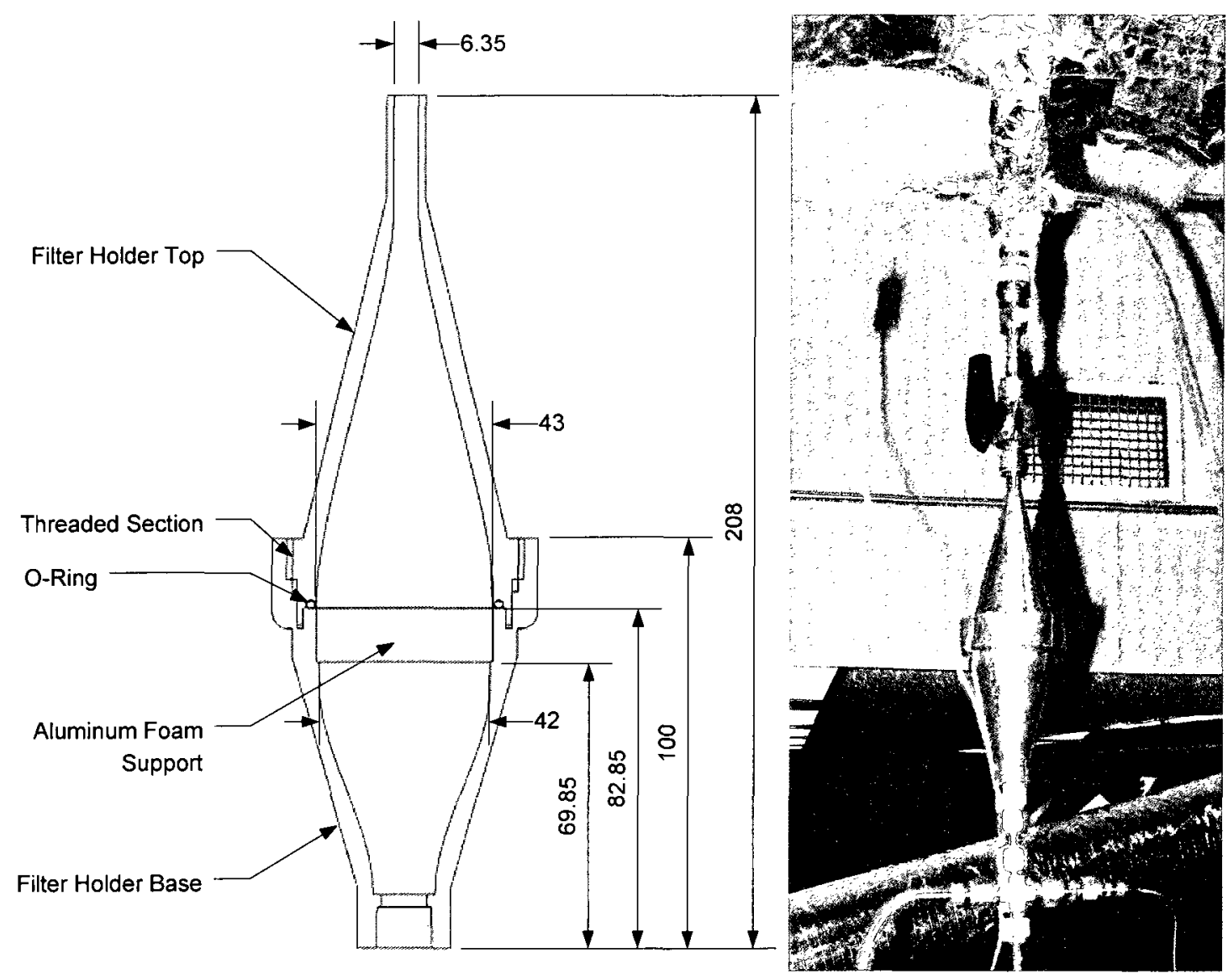

Figure 3.10 - Schematic of filter holder and photograph of filter assembly, pictured here without the secondary dilution device (all dimensions in $\mathbf{m m}$ ).

The filter was supported in the holder by a $12.7 \mathrm{~mm}$ thick piece of sintered nickel foam, counter-sunk into the holder to provide a flush surface to support the filter. The foam support was $45 \mathrm{~mm}$ in diameter, leaving a $1 \mathrm{~mm}$ annular ring of metal as a sealing surface. The seal was achieved using a $2 \mathrm{~mm}$ thick o-ring. The two halves of the filter 
holder were threaded, and when assembled, compressed the o-ring onto the filter creating a seal between the filter and the seal surface. This seal ensured that no gas or particles could bypass the filter. Losses were minimized by avoiding sharp contractions, expansions, or abrupt changes of area in the filter holder.

The positive seal of the filter holder was checked following the procedure set forth by the Air Resources Board (ARB) of California [ARB, 2008]. In this method, the intake nozzle (inlet of the quick-connect) was plugged and the vacuum pump turned on. The vacuum pressure was adjusted to 15 inches of mercury and after the pressure gauge had settled, the flow rate was monitored to ensure it remained below 0.566 LPM during a $30 \mathrm{~s}$ period. If the leak rate was found to be satisfactory, the plug was removed and testing commenced. This test method is used as a quality assurance technique for measurements taken following the USEPA EMC [2000] test method and is thus considered appropriate here.

Photographs of filters deposited with soot are shown in Figure 3.11. The images show that the filter holder evenly distributes the sample across the filter face, and the white ring around the edges shows that the o-ring is providing the positive seal necessary to prevent leakage. The white dots and lines apparent on the filter are due to the support material downstream of the filter, which prevents gas flow through the filter at these locations. 


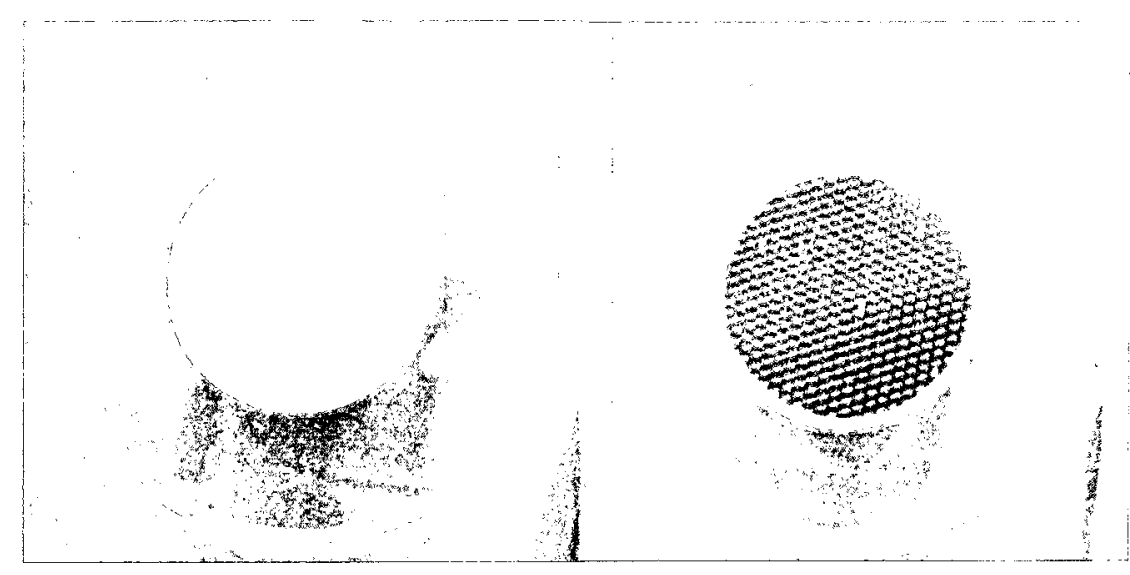

Figure 3.11 - Photographs of filters deposited with small (left) and large (right) amounts of soot

Flow through the filter surface was measured using a mass flow controller (Brooks Smart series $5851 \mathrm{~S} / \mathrm{BD})$. This flow rate and all other parameters were recorded every 0.5 seconds by a LabVIEW program that controlled the experiment. The total volume of exhaust sample drawn through the filter was calculated as the summation of each recorded flow rate multiplied by the time step as shown in Equation 3.7. For the case where secondary dilution was used, $V_{\text {sample }}$ was found using the continuity equation, where the sample flow rate was calculated as the difference of the total flow rate and the secondary dilution flow rate (controlled via Brooks Smart series model 5850S/BD) corrected to dilution tunnel temperatures as shown in Equation 3.8:

$$
\begin{gathered}
V_{\text {sample }}=\sum_{t=0}^{t=N}\left[Q_{\text {sample }, t} \cdot 0.5\right] \\
V_{\text {sample }}=\sum_{t=0}^{t=N}\left[\left(\dot{m}_{\text {total }}-\dot{m}_{\text {dilution }}\right) \frac{R_{u} \cdot T_{D T}}{P_{s} \cdot M W} \cdot 0.5\right]
\end{gathered}
$$

where $N$ is the duration of the test, $P_{s}$ is the static pressure in the dilution tunnel, and $T_{D T}$ is the temperature at the centreline of the dilution tunnel. The sample was drawn using a GAST Motor Mounted Rotary Vane oil-less vacuum pump, model 1423 (25" Hg maximum vacuum, 13.2 CFM open flow). 


\subsubsection{Filter Selection}

For the present work, typical soot aggregate primary particle sizes are in the range of 30-

$51 \mathrm{~nm}$. Although aggregate sizes were not directly measured, Canteenwalla [2007] measured aerodynamic diameters of less than $1 \mu \mathrm{m}$ for soot generated under similar conditions using a scanning mobility particle sizer (SMPS), and detailed soot morphology measurements by Köylü and Faeth [1992] for similar flames burning propane noted that primary particles were clustered into aggregates with an average of 226 particles per aggregate.. Due to their small size, care was exercised in selecting a filter which would efficiently trap all sizes of soot aggregate while also allowing sufficient face velocity for a timely acquisition of sufficient soot for gravimetric analysis. There is no established standard for filtering soot as demonstrated by the range of filter diameters and material types suggested from the standards consulted (Table 3.6).

Table 3.6 - Comparison of Filter requirements

\begin{tabular}{|c|c|c|}
\hline Protocol & Filter size (dia.) & Filter Material \\
\hline Current Setup & $47 \mathrm{~mm}$ & Millipore Fluoropore PTFE membrane \\
\hline USEPA EMC [2000] & N/A & glass fibre filters \\
\hline USEPA [2005] 40 CFR Part 1065 & $47 \mathrm{~mm}$ & PTFE-coated glass fibre filter \\
\hline UNECE [2008] regulation 49 & $47-70 \mathrm{~mm}$ & fluorocarbon glass fibre \\
\hline
\end{tabular}

The work of John and Reischl [1978] provides a basis for comparing filter efficiencies for several material types and brands. Their work showed that glass fibre filters typically have a higher filtration efficiency compared to cellulose membrane filters or nuclepore filters. It also showed that polytetrafluoroethylene (PTFE) filters have filtration efficiencies comparable to glass fibre filters, the filtration efficiency increases with decreasing rated pore size, and the filtration efficiency is relatively unaffected by changes in the filter face velocity. The tests of John and Reischl [1978] for PTFE filters 
were conducted on Millipore Fluoropore $47 \mathrm{~mm}$ diameter filters with rated pore sizes ${ }^{1}$ of $3 \mu \mathrm{m}$ and $1 \mu \mathrm{m}$. The filter efficiencies for the $3 \mu \mathrm{m}$ pore filter range from 97 to $>99.9 \%^{2}$, while the efficiency for the $1 \mu \mathrm{m}$ pore filter was always $>99.9 \%$, regardless of filter face velocity. Since filter efficiency increases with decreasing pore size, a Fluoropore PTFE filter with a rated pore size of $0.22 \mu \mathrm{m}$ was used in the present work. The decreased pore size should only increase the filtration efficiency and still allows for filter face velocities in the range suggested by Brock [1983] of $1 \mathrm{~cm} / \mathrm{s}<u_{\text {face }}<100 \mathrm{~cm} / \mathrm{s}$.

The filter handling procedures before and after tests are equally important for ensuring consistent, reliable results. The temperature and relative humidity of the conditioning environment as well as the electrostatic charge build-up on the filter can affect the measured mass of the filters if they are not properly controlled [Tsai $e t$ al., 2002; Taylor, 2006]. In the tests of Tsai et al. [2002], four different materials were tested; glass fibres, mixed cellulose ester (MCE) filters, polyvinyl chloride (PVC) filters, and Teflon filters. They concluded that the hygroscopicity of the filter determines the extent to which temperature and relative humidity affect the measured filter mass. The MCE filters were affected most severely by relative humidity and were known to be hydrophilic. They therefore recommended the use of filters that are hydrophobic in nature for aerosol sampling. Although they did not specifically test PTFE filters, the Fluoropore PFTE membrane filters are hydrophobic. Tsai et al. [2002] also suggested that neutralizing the electrostatic charge of the filters before measurement could increase the precision of the results. In the present work, each filter was neutralized using an

\footnotetext{
${ }^{1}$ Rated pore size refers to the size (diameter) of the largest pore.

${ }^{2}$ In the work of John and Reischl [1978], the apparatus was able to detect particles as small as $0.0025 \mu \mathrm{m}$ and the efficiencies are considered valid down to particles of this size.
} 
alpha ionizing $\mathrm{Po}^{210}$ electrical charge neutralizer (NRD Staticmaster, model 2U500) prior to weighing.

The filter diameters suggested by the consulted standards were in the range of 47-70 $\mathrm{mm}$ diameter. A $47 \mathrm{~mm}$ diameter filter was selected for the present work because: 1) the work of John and Reischl [1978] was completed on $47 \mathrm{~mm}$ filters, 2) the Fluoropore $47 \mathrm{~mm}$ filters allows for the required sample flow rates and filter face velocities, and 3) this size of filter was easier to handle and required a smaller filter holder than a $70 \mathrm{~mm}$ filter. The Millipore Fluoropore PTFE membrane filter with a diameter of $47 \mathrm{~mm}$ and a rated pore size of $0.22 \mu \mathrm{m}$ (Millipore model number FGLP04700) was selected for the current work.

\subsubsection{Filter Handling}

To maximize the precision of the gravimetric measurements, filters were conditioned in a room of fixed temperature and relative humidity. The exact temperature and relative humidity of the conditioning room were monitored as part of the sampling protocol. Table 3.7 shows the different conditions as suggested by the standards mentioned previously and the conditions implemented in the current setup. Included in this table is the USEPA TP 714C [1994] sampling protocol in addition to the previously mentioned protocols. USEPA TP 714C was the standard followed in the work of Tsai et al. [2002], and is the standard used to control the set points of the clean room facilities used at the NRC. Of specific importance is the pre-test to post-test change in conditions, noted by the \pm after the temperature and relative humidity in Table 3.7. The tight controls on the environmental conditions ensure no moisture or buoyancy biases are incurred. Each filter was conditioned in the climate-controlled clean room for a minimum of 8 hours before a 
pre-test measurement, and for 8-56 hours for a post-test measurement. Filters were weighed using a Mettler Toledo electronic microbalance (model UMX2), which had a resolution of $0.1 \mu \mathrm{g}$ and was isolated from vibrations with an air suspension isolation table (Kinetic Systems Inc., Vibraplane). As stated above, in the present work, each filter was neutralized using an alpha ionizing $\mathrm{Po}^{210}$ electrical charge neutralizer (NRD Staticmaster, model 2U500) prior to weighing.

Table 3.7 - Comparison of conditioning requirements

\begin{tabular}{|c|c|c|c|c|c|}
\hline Protocol & $\begin{array}{c}\text { Conditioning } \\
\text { room temp. }\end{array}$ & $\begin{array}{c}\text { Conditioning } \\
\text { room dew } \\
\text { point }\end{array}$ & $\begin{array}{c}\text { Conditioning } \\
\text { room R.H. }\end{array}$ & $\begin{array}{c}\text { Time for pre } \\
\text { conditioning }\end{array}$ & $\begin{array}{c}\text { Time for } \\
\text { post } \\
\text { conditioning }\end{array}$ \\
\hline Current Setup & $20-27 \pm 2^{\circ} \mathrm{C}$ & N/A & $35-68 \pm 10 \%$ & 8 hours & $8-56$ hours \\
\hline $\begin{array}{c}\text { USEPA EMC } \\
{[2000]}\end{array}$ & N/A & N/A & N/A & N/A & N/A \\
\hline $\begin{array}{c}\text { USEPA [2005] 40 } \\
\text { CFR Part 1065 }\end{array}$ & $22 \pm 1^{\circ} \mathrm{C}$ & $9.5 \pm 1^{\circ} \mathrm{C}$ & N/A & 30 min. & 60 min. \\
\hline $\begin{array}{c}\text { UNECE [2008] Reg. } \\
49\end{array}$ & $22 \pm 3^{\circ} \mathrm{C}$ & $9.5 \pm 3^{\circ} \mathrm{C}$ & $45 \pm 8 \%$ & 60 min. & 8 hours max. \\
\hline $\begin{array}{c}\text { USEPA [1994] TP } \\
714 \mathrm{C}\end{array}$ & $20-30 \pm 6^{\circ} \mathrm{C}$ & N/A & $30-70 \pm 10 \%$ & 8 hours & $1-56$ hours \\
\hline
\end{tabular}

Eight filters were used for each test condition, although some would be discarded due to contamination or breakage. This included one control filter per test, which was handled in the same manner as the other filters, but was not used to collect soot. This control filter was used to detect any impact the handling procedures may have on the measured soot mass (typically less than $2 \%$ of soot mass) and each test was adjusted by this offset. Two blank control filters were kept in the clean room environment at all times and were used to detect any drifting of the balance or clean room environmental conditions as recommended by USEPA TP 714C [1994]. This test procedure recommends that if the value of these control filters should change by more than $\pm 2.0 \%$ of the test average primary filter loading (i.e. soot mass) from pre-test to post-test, that the samples be discarded and the test repeated. A control filter deposited with soot was 
also monitored long term to ensure that the mass of deposited soot did not vary with time. Figure 3.12 shows the variation in mass for all three control filters with time. The mean, standard deviation, and standard deviation of the means for the control filters are given in Table 3.8. These control filters were also used to verify that filter and soot mass were not deviating with temperature or relative humidity as shown in Figure 3.13. The slope of the linear curve-fit of blank filter 1 for the temperature and relative humidity plot is $\cdot-2.072 \times 10^{-5} \mathrm{mg} /{ }^{\circ} \mathrm{C}$ and $-5.259 \times 10^{-5} \mathrm{mg} / \%$ R.H., respectively, both negligible amounts over the range of conditions experienced. Similar trends were found for the second blank filter and the sooty control filter.

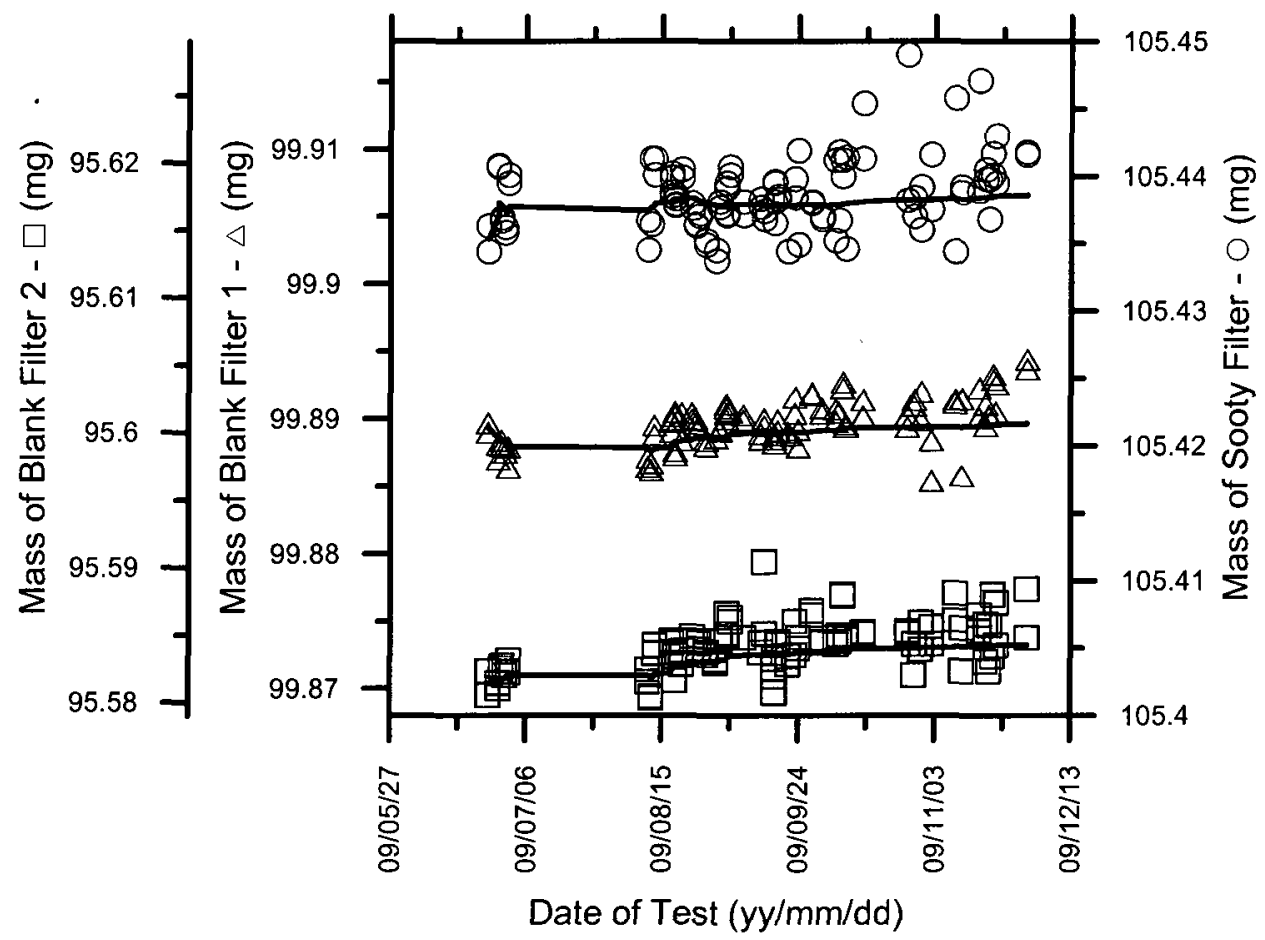

Figure 3.12 - Mass of control filters with time. Key to symbols on Y-axes. Solid black lines represent the cumulative averages.

Table 3.8 - Mean, standard deviation, and standard deviation of the means of control filters

\begin{tabular}{|c|c|c|c|}
\hline Filter & $\begin{array}{c}\text { Average } \\
\text { Mass (mg) }\end{array}$ & $\begin{array}{c}\text { Standard } \\
\text { Deviation (mg) }\end{array}$ & $\begin{array}{c}\text { Standard Deviation } \\
\text { of the Mean }\end{array}$ \\
\hline Blank 1 & 99.8895 & 0.0015 & 0.00016 \\
\hline Blank 2 & 95.5841 & 0.0018 & 0.00019 \\
\hline Sooty Filter & 105.4383 & 0.0026 & 0.00028 \\
\hline
\end{tabular}




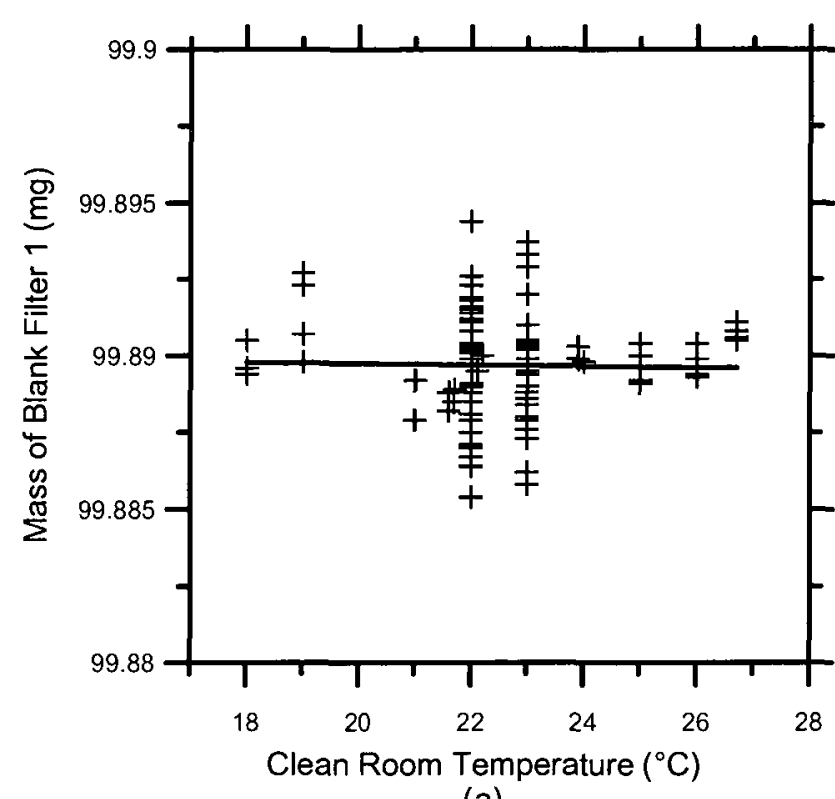

(a)

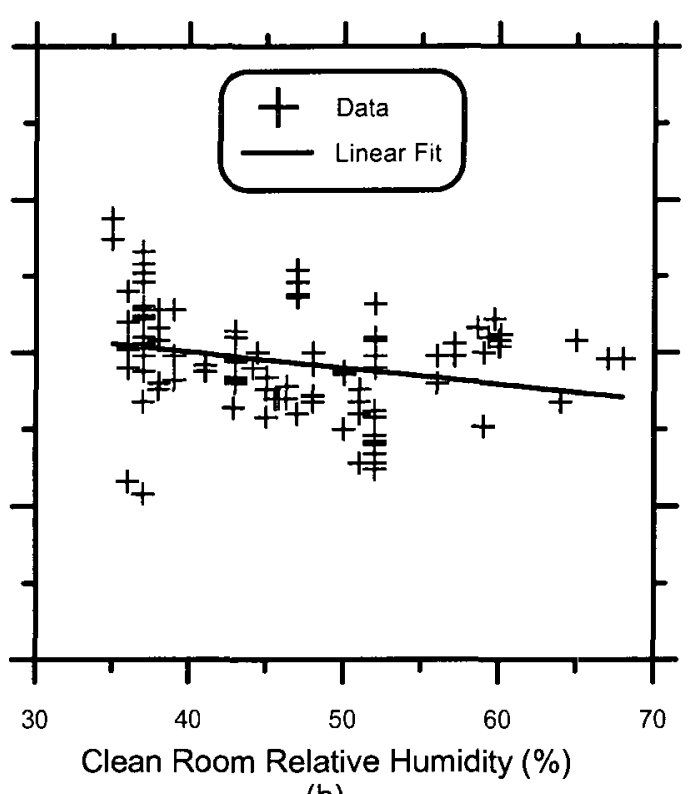

(b)

Figure 3.13 - Variation of blank filter 1 mass with (a) temperature and (b) relative humidity

\subsubsection{Laser Induced Incandescence}

LII is a recently-developed technique used for measuring the soot volume fraction in a gas. The reader is referred to Snelling et al. [2005] for a detailed description of the theory behind a commercial version of the apparatus manufactured by Artium Technologies (model LII200) which was used for the tests performed in this work. The following quote was reproduced from the manufacturer's brochure for a brief description:

"In simple terms, the technique employs a pulsed laser with light pulse duration below 20 nanoseconds which is used to rapidly heat the soot particles from the local ambient temperature to close to the soot vaporization temperature $(2500$ to $4500 \mathrm{~K})$. Incandescence from the heated soot particles is detected by photodetectors, and the signals are recorded for subsequent analyses. Analysis involving the laser light energy absorption by the soot particles, and the subsequent cooling process, is used to calculate the soot volume fraction and primary particle size. The main advantages of this technique are that it provides real time measurements, has a large dynamic range, and only measures the black carbon particulate. At the high temperatures experienced, all volatile components that may have been condensed on the soot particles will be promptly evaporated, and most other non-refractory particles will have evaporated or undergone sublimation. Due 
to this selectivity, LII does not measure the total particle mass, but the total soot particle mass."

It is therefore expected that the soot volume fraction determined by gravimetric analysis will be slightly higher due to the presence of these non-evaporated or unsublimated volatile particles.

The LII200 system uses the self-calibrating LII methodology as described in Snelling et al. [2005]. Briefly, absolute intensity calibrated soot incandescent emission is measured at two wavelengths by photomultiplier tubes equipped with narrowband interference filters $(400 \mathrm{~nm}, 780 \mathrm{~nm})$. From the measurement of incandescence at two wavelengths, the soot particle temperature and soot volume fraction are determined. Figure 3.14 shows a schematic of the LII system used in the experiment and Equation 3.9 shows the general formula for calculation of the soot volume fraction:

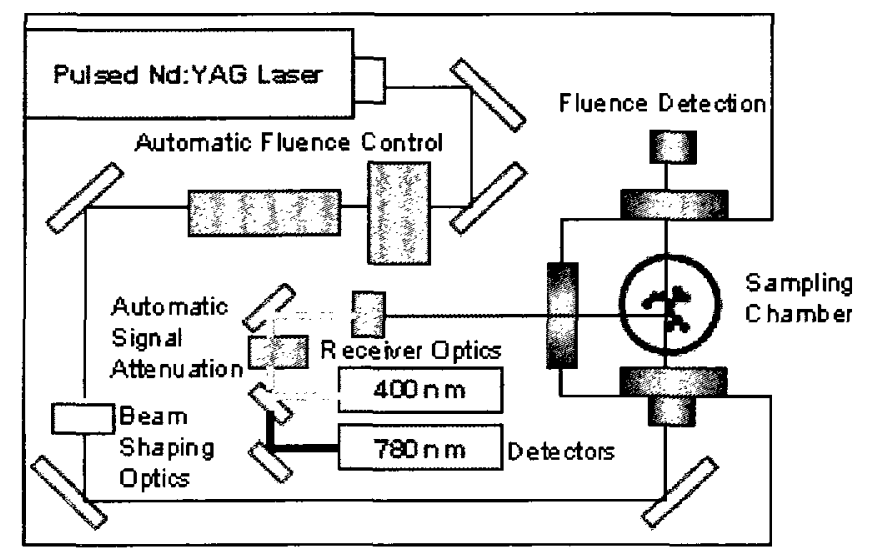

Figure 3.14 - Schematic of Artium LII system [Canteenwalla, 2007]

$$
f_{v}=\frac{V_{E X P}}{\eta \omega_{b} G_{E X P} \frac{12 \pi c^{2} h}{\lambda_{c}^{6}} E\left(m_{\lambda_{c}}\right)\left[\exp \left(\frac{h_{p} c}{k \lambda_{c} T_{p}}\right)-1\right]^{-1}}
$$

where $V_{E X P}$ is the experimental voltage signal

$\eta \quad$ is the calibration factor

$\lambda \quad$ is the wavelength

$\omega_{b} \quad$ is the laser beam thickness 
$G_{E X P}$ is the experimental detector gain

$c \quad$ is the speed of light

$h_{p} \quad$ is the Planck constant

$k \quad$ is the Boltzmann constant

$E\left(m_{\lambda}\right)$ is the absorption function

$T_{p} \quad$ is the particle temperature

\subsubsection{Sampling Train}

The sampling train employed in the LII measurement was nearly identical to that described for the gravimetric process except for two main differences: the LII instrument was installed between the filter holder and the inlet temperature measurement location and the LII instrument was connected to the sample probe via a $3 \mathrm{~m}$ heated sample line (Technical Heaters, Inc. Series 212 Model 10 feet). The measurement cell of the LII device was also heated, and both of these components (cell and sample line) were maintained at the recommended temperature of $338 \mathrm{~K}$ to reduce the impact of thermophoretic losses in the sampling train. A schematic of the setup is shown in Figure 3.15. A filter was installed at the outlet of the LII to remove soot particles which could damage the thermal sensor inside the mass flow controller downstream.

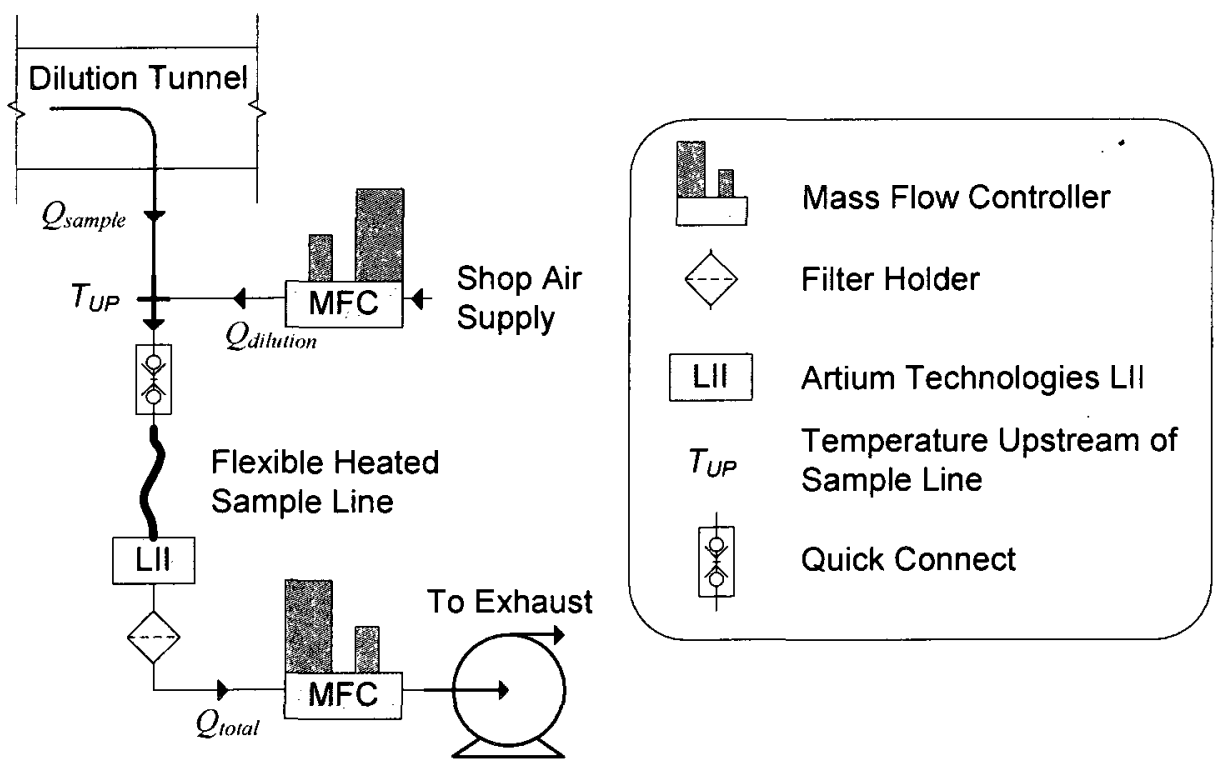

Figure 3.15 - Schematic of LII sampling train 


\subsection{Flame Imaging}

Flame length was measured using the method described in detail by Canteenwalla [2007], which is based on the work of Kostiuk et al. [2000]. The technique involved analysis of video images ( $720 \times 480$ pixels interlaced, $30 \mathrm{fps}$ ) of the luminous flame, converted to digital files on a computer using a LabVIEW routine. A mean image was created by averaging over 140 frames from a 120 second period. From the mean flame image, the flame length was arbitrarily defined as the distance from the burner exit tip to the $10 \%$ contour line. The reader is referred to Canteenwalla [2007] for a detailed description of the LabVIEW code routine used to calculate the length.

\subsection{Sampling Protocol}

Due to the large number of test conditions, a standard protocol was developed to ensure repeatable results. Of specific importance were the warm-up time, DT conditions, and sampling duration. Additionally, it was necessary to clean the optical windows of the LII200 prior to each test session due to observed deposition of soot for some test conditions. The windows were cleaned with isopropanol prior to each test session.

The warm-up time was determined by monitoring several different temperature measurements on start-up, including the gas temperatures at the burner exit, inside the enclosure, in the DT, and upstream of the filter or heated sampling line. Once these temperatures reached stable values (generally around $\sim 30$ minutes), testing would commence.

Sampling losses were deemed negligible based on the detailed analysis of the LSF sampling system by Canteenwalla [2007]. Details of this analysis can be found in 
Appendix $\mathrm{C}$ of his work. He concluded that the sampling efficiency was typically greater than $97 \%$, and therefore it should be expected that the sampling losses in the system are not greater than $3 \%$.

The DT conditions were discussed in more detail in section 3.2.2, but the most critical aspect was that of the gas temperatures directly upstream of the filter. The fan speed could be changed to increase or decrease the amount of primary dilution air required to cool the exhaust gas to the desired temperature. If the primary dilution air alone was not sufficient to cool the exhaust gases to the desired temperature, the secondary dilution device was activated to add a controlled amount of shop air to the sample. Although control of the exhaust gas temperature was not deemed necessary for LII operation it was decided that conditions for measurements should be identical between gravimetric and LII tests.

The temperature control was chosen as a replacement of the DR control used by Canteenwalla [2007] following the review of relevant standards summarized in Table 3.5 and discussed in Section 3.2.2 above. In his work, Canteenwalla found that the soot yield varied depending on the DR, leading to a correction protocol based on a standard DR of 50. To ensure that the DR was not having an affect using the constant temperature protocol, several tests were carried out using the $25.4 \mathrm{~mm}$ burner at $0.5 \mathrm{~m} / \mathrm{s}$ burning the average 6-component fuel mixture. The fan speed was varied from $20-100 \%$ while the temperature upstream of the sample location was held to $<52^{\circ} \mathrm{C}$ by use of the secondary dilution device. The results of these tests for both gravimetric and LII results are shown in Figure 3.16, and show negligible differences at the different fan speeds used, establishing that the current protocol is satisfactory. During these tests the sample flow 
rate changes based on the amount of secondary dilution added, which again shows that isokinetic sampling is not necessary as the change in the strength of the sink (the sample probe) in the DT does not affect the soot yield.

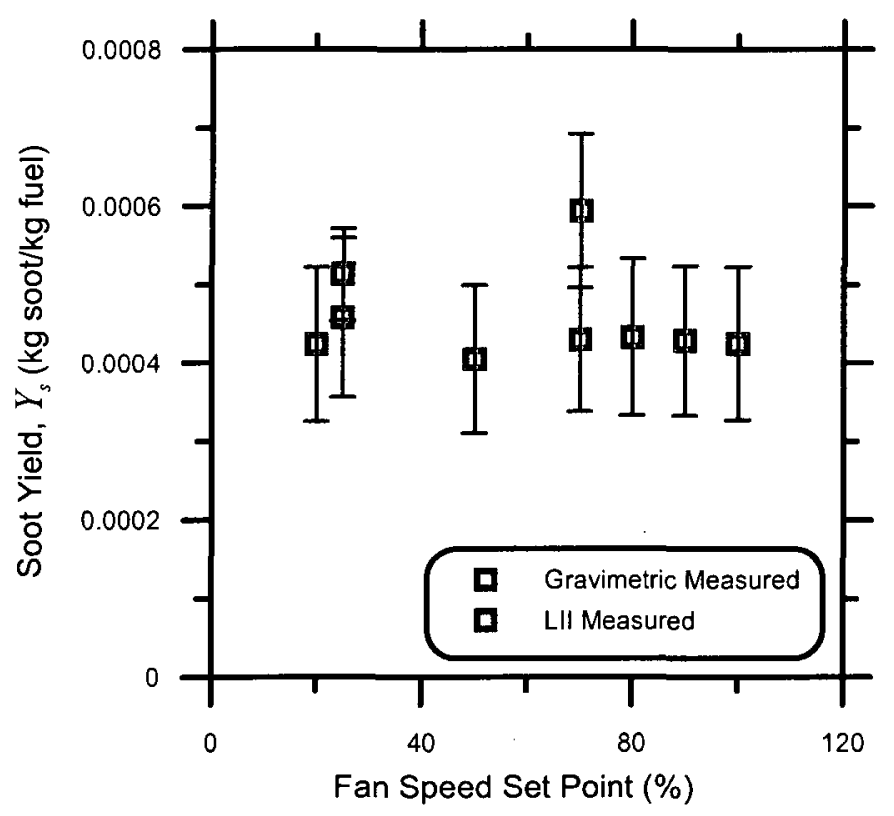

Figure 3.16 - Soot yield as a function of the DT fan speed for tests using the $25.4 \mathrm{~mm}$ burner using the average 6-component fuel mixture at 15.2 SLPM

The sampling duration for gravimetric tests was based on the minimum amount of sample required to ensure reasonable uncertainties. It was determined that a minimum of $50 \mu \mathrm{g}$ of soot should be obtained for each test to ensure reasonable uncertainties $(<\sim 15 \%)$. By basing the test time on the amount of soot required, this meant that the test time could vary between five minutes for heavy sooting conditions to one hour for low sooting conditions.

Test duration for the LII tests was determined via a convergence criterion applied to the time resolved data being recorded. Figure 3.17 shows a typical LII test, including the instantaneous data points, the cumulative average, and the upper and lower bounds of the final uncertainty (to be described in detail in section 3.7). As can be seen, there is a general settling of the bounds of uncertainty defined by the component contributors to 
uncertainty, namely the systematic and precision uncertainties. Eventually, the precision uncertainty becomes small in comparison to the systematic uncertainty, and more data will not lead to a reduction in the estimated uncertainty. The confidence interval $(95 \%$ confidence level) of the test is calculated and a test will be considered complete if this value is less than $0.5 \%$ of the mean, and the calculated uncertainty and average become stable. The choice of $0.5 \%$ is somewhat arbitrary; however testing has shown this to be a reasonable protocol for producing repeatable results.

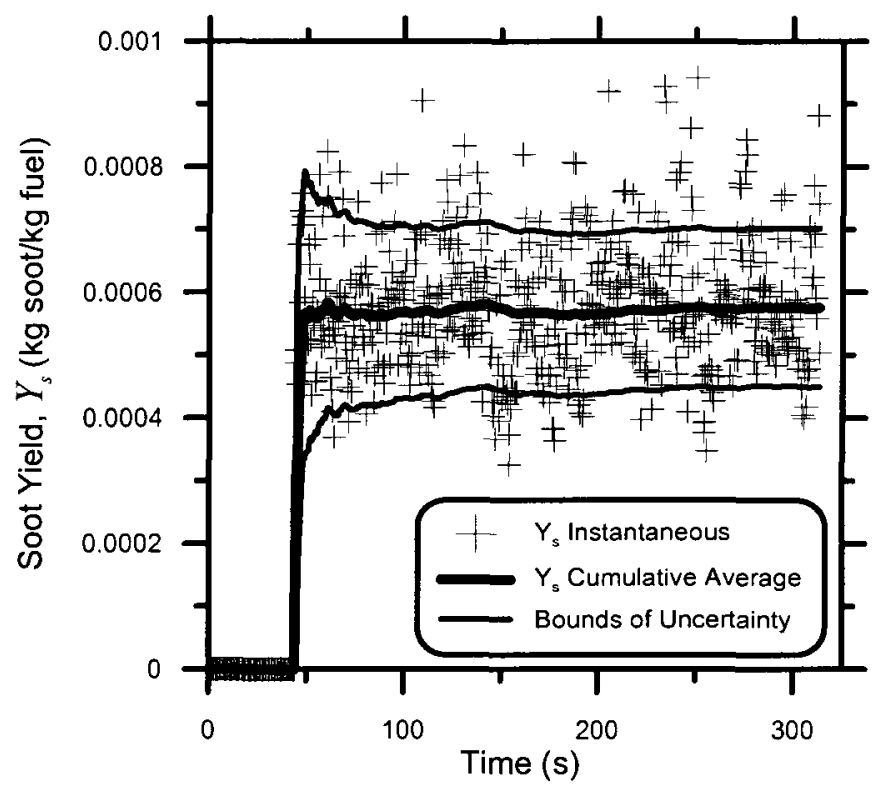

Figure 3.17 - Typical soot yield data for a test with the $25.4 \mathrm{~mm}$ burner using the average 6component fuel at 15.2 SLPM

During LII operation, anomalous soot volume fraction data points (1-2 orders of magnitude above the average) were occasionally observed. These erroneous data points resulted in large confidence intervals and were most likely due to large dust particles in the room air or possibly large soot particles deposited on the DT or sampling line walls being re-entrained into the flow. Chauvenet's criterion was used to filter these erroneous 
points (Figure 3.18) from the soot volume fraction data output from the LII instrument ${ }^{1}$. Chauvenet's criterion will discard a data point if the probability of obtaining the particular deviation from the mean is less than $1 /(2 n)$, where $n$ is the total number of sample points.

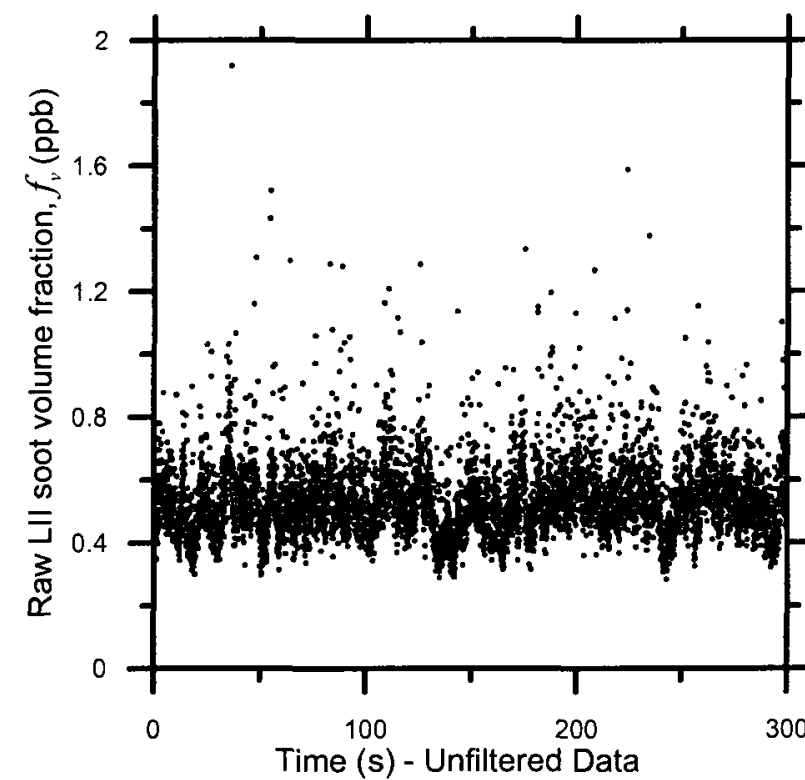

(a)

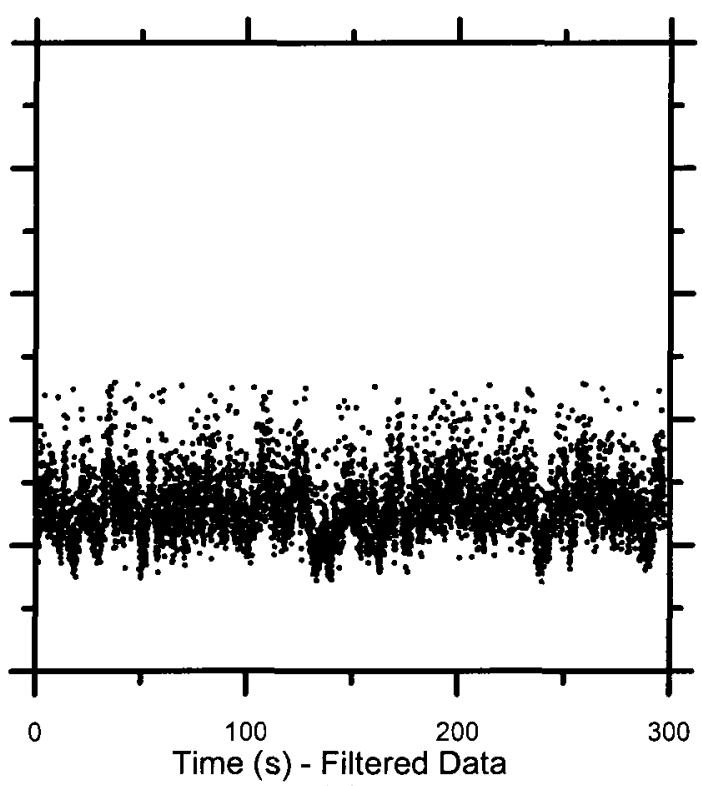

(b)

Figure 3.18 - Comparison of LII test (a) with and (b) without the points deemed outliers by Chauvenet's Criterion

\subsection{Soot Factor Equations}

The simplest and most common form of emission factor is based on the emitted mass of a pollutant per mass or volume of combusted fuel. The current factor given in the NPRI guide [CAPP, 2007] defines the emission factor in units of $\mathrm{kg}$ soot $/ 10^{3} \mathrm{~m}^{3}$ fuel. While volumes of gas are more readily measured in the flaring industry, the composition and density of the gas can vary from site to site, making a mass-based emission factor more practical. The soot yield, $Y_{s}$, has been defined as the mass of soot per mass of fuel

\footnotetext{
${ }^{1}$ Calculation of the soot yield from the unfiltered soot volume fraction data resulted in an average increase of $\sim 1 \%$, a negligible amount in the context of the overall uncertainty as shown in section 3.7
} 
burned. Another form of this equation used in the literature [Sivathanu and Faeth, 1990; Becker and Liang, 1982] is the soot generation efficiency ( $S G E)$, defined as the mass of soot per mass of fuel-bound carbon.

Since it was deemed impractical to collect all of the soot generated from the dilution tunnel, the mass of soot was determined by measuring a representative sample as discussed in Section 3.3. The soot volume fraction, $f_{v}$, which was measured by either gravimetric or LII techniques, was multiplied by the volume flow in the DT $\left(Q_{D T}\right)$ to determine the volumetric flow of soot in the DT $\left(Q_{\text {soot }}\right)$. The soot volume fraction in a sample drawn from the DT was defined as $f_{v}=V_{\text {soot }} / V_{\text {sample }}$, where $V_{\text {soot }}$ was the volume of soot within the sample volume, $V_{\text {sample. }}$ For iso-kinetic conditions, or when iso-kinetic conditions are not necessary, this definition of the volume fraction is equivalent to $f_{v}=Q_{\text {soot }} / Q_{D T}$, where $Q_{\text {soot }}$ and $Q_{D T}$ are the volume flow rates of soot and exhaust gases through the DT.

It was shown in section 3.2.2 that iso-kinetic conditions were not necessary to achieve unbiased sampling of the soot particles, most likely because the particles were very small and readily tracked the flow. Assuming that all the soot measured was generated from the flare, the mass emission rate of soot could be determined from Equation 3.10.

$$
\dot{m}_{\text {soot }}=\rho_{\text {soot }} f_{v} Q_{D T}
$$

This assumption was valid as background measurements made with the LII instrument detected no measureable soot volume fraction, with an instrument detection limit of $0.001 \mathrm{ppb}$, when the LSF was not operating. The soot density can be assumed constant with temperature, but the exhaust gas densities can not. Assuming ideal gas behaviour, 
the measured soot volume fraction at the sample location was related to conditions in the dilution tunnel via Equation 3.11.

$$
f_{v, D T}=f_{v, \text { sample }}\left(T_{\text {sample }} / T_{D T}\right)
$$

Combining Equations 3.10 and 3.11, the general form of the soot yield equation is given in Equation 3.12.

$$
Y_{s}=\frac{\dot{m}_{\text {soot }}}{\dot{m}_{\text {fiel }}}=\frac{\rho_{\text {soot }} f_{v, \text { sample }} Q_{D T} T_{\text {sample }}}{\dot{m}_{\text {fitel }} T_{D T}}
$$

For LII tests, the general form of Equation 3.12 was used, where $f_{v \text {,sample }}$ was the soot volume fraction measured by the LII and $T_{\text {sample }}$ was the temperature of the LII sample cell. In cases where secondary dilution was added, the volume fraction measured by the LII needed to be corrected by the addition of air via Equation 3.13.

$$
f_{v, \text { sample }}=f_{v, L I I}\left(\frac{\dot{m}_{\text {total }}}{\dot{m}_{\text {total }}-\dot{m}_{\text {dilution }}}\right)
$$

Combining this with the general form of the soot yield equation yields Equation 3.14.

$$
Y_{s, L I I}=\frac{\rho_{\text {soot }} f_{v, L I I} Q_{D T} T_{\text {sample }}}{\dot{m}_{\text {fitel }} T_{D T}}\left(\frac{\dot{m}_{\text {total }}}{\dot{m}_{\text {total }}-\dot{m}_{\text {dilution }}}\right)
$$

For gravimetric tests, the soot volume fraction was calculated using Equation 3.6, which when substituted into Equation 3.12 yielded Equation 3.15.

$$
Y_{s, \text { Grav }}=\frac{m_{\text {soot }} Q_{D T}}{V_{\text {sample }} \dot{m}_{\text {fiuel }}}
$$

It should be noted that no temperature correction was needed in Equation 3.15 because the sample volume, $V_{\text {sample, }}$ was already corrected to the dilution tunnel conditions based on ideal gas behaviour. 
Once the soot yield was determined, it could easily be converted to the other forms of soot factors mentioned previously such as:

$$
\begin{gathered}
S G E=Y_{s} \frac{\dot{m}_{\text {fiel }}}{\dot{m}_{\text {carbon }}} \\
E F=Y_{s} \rho_{\text {fuel }} \cdot 1000
\end{gathered}
$$

where $\dot{m}_{\text {carbon }}$ is the mass flow rate of fuel bound carbon, $\rho_{\text {fuel }}$ is the fuel density, and $E F$ is the form of soot emission factor used in the CAPP guide [CAPP, 2007].

\subsection{Uncertainty Analysis}

A detailed uncertainty analysis was conducted based on the ANSI/ASME Measurement Uncertainty Standard [1985], which considers separate contributions of the systematic error (alternatively called "bias error" or "instrument accuracy"), denoted by $B$, and the precision error (or random error), denoted by $P$, to the total uncertainty. The systematic error is the inability of the equipment to measure the correct value. Each instrument will have its own associated systematic error, which is propagated through to the final measurement. It is assumed in the uncertainty standard that the systematic uncertainties encountered are normally distributed. The precision error is the scatter in repeated measurements caused by random error. Each component error is estimated separately and then combined into a final uncertainty, $U$, in quadrature.

The overall systematic error in $Y_{s}$ is a combination of the systematic errors of all the components used in calculating its value. For a general function $g$ of multiple variables $\mathrm{x}_{i}$ where $i=1,2, \ldots \mathrm{L}$, the systematic error, $B$, of each measured variable is combined as:

$$
B_{g}=\left(\sum_{i=1}^{L}\left[\theta_{i} B_{x_{i}}\right]^{2}\right)^{1 / 2}
$$


where $B_{g}$ is the final systematic uncertainty of $g, B_{x i}$ is the systematic uncertainty of each variable $x_{i}$, and $\theta_{i}$ is the sensitivity index defined in Equation 3.19.

$$
\theta_{i}=\frac{\partial g}{\partial x_{i_{x=\bar{x}}}} i=1,2, \ldots L
$$

The precision error is a measure of the scatter in the results of different tests for the same operating condition. This is calculated by multiplying the standard error of the sample average by the appropriate $95 \%$ confidence interval $t$-value from the Student's t-distribution table as shown in Equation 3.20:

$$
P=t_{v, 95 \%} \frac{\sigma}{\sqrt{N}}
$$

where $\sigma$ is the sample standard deviation, $N$ is the sample size, and the subscript $v$ is the degrees of freedom $(N-1)$. The systematic and precision errors are assumed independent and combined in quadrature by the root sum of the squares method to produce an approximate total uncertainty, $U$, as,

$$
U=\sqrt{B^{2}+P^{2}}
$$

The precision error is only calculated at the final stage of uncertainty analysis as it is assumed that the scatter in contributing components of the final uncertainty will be propagated to the scatter of the final calculated value. For example, if $\bar{g}$ is an average of $N$ measurements of $g$, and $g$ is a function of $x$ and $y$, each of which have an associated precision error, the final precision of $\bar{g}$ is calculated as the scatter on $g$, where the precision error will reflect both precision errors within tests, and variation between tests. 


\subsubsection{Systematic Gravimetric Uncertainty}

Examination of Equation 3.15 shows that there are four components of the soot yield equation for gravimetric analysis. Each is discussed in detail in separate sections below.

\subsubsection{Fuel Mass Flow Rate}

The total fuel mass flow rate can be a single measurement (i.e. in the case of a pure fuel) or a combination of up to 6 measurements (i.e. for 6-component fuel mixtures). The total systematic uncertainty will be the combination of the individual uncertainties of each mass flow controller. Since the total mass flow rate of fuel was calculated as the sum of all the fuel components, the sensitivity indices each had a value of one, and the total fuel mass flow rate uncertainty was calculated as shown in Equation 3.22. A typical value for the systematic uncertainty of the total fuel mass flow rate of a 6-component fuel mixture was $1.3019 \times 10^{-5} \mathrm{~kg} / \mathrm{s}$.

$$
B_{\dot{m}_{f_{\text {fuel }}}}=\sqrt{B_{\dot{m}_{1}}^{2}+B_{\dot{m}_{2}}^{2}+B_{\dot{m}_{3}}^{2}+B_{\dot{m}_{4}}^{2}+B_{\dot{m}_{5}}^{2}+B_{\dot{m}_{6}}^{2}}
$$

\subsubsection{Mass of Soot}

To determine the systematic uncertainty in the calculation of the mass of soot collected on a filter, the uncertainty of the microbalance had to be determined. This was done by combining (by root of the sum of the squares) the elemental uncertainties given in the product manual. The repeatability $(0.00025 \mathrm{mg})$ and linearity $(0.001 \mathrm{mg})$ were combined to produce an instrument systematic uncertainty of $0.00103 \mathrm{mg}$. Since the mass of soot was the difference of two measurements of filters, the instrument systematic error was multiplied by $\sqrt{ } 2$ to give a soot mass systematic uncertainty of $0.00146 \mathrm{mg}$. 


\subsubsection{Dilution Tunnel Flow Rate}

The calculation method for the DT flow rate utilized numerical integration to find the total flow rate. The typical method to find the error in a numerical integration would be to find the difference between the actual integral and the value calculated by the numerical method. Since an actual integral can not be calculated from the discrete data points, the systematic uncertainty of the dilution tunnel flow rate was found by the ANSI/ASME [1985] method. The numerical integration was based on the velocity at several points in the DT. Each velocity had its own associated uncertainty based on the velocity at that location and the range of the pressure transducer used with the corresponding Pitot tube. The velocity was calculated based on the differential pressure of the Pitot tube and the density of the gas as shown in Equation 3.2. Each of these measurements had its own elemental uncertainties as shown below.

The differential pressure was determined directly from pressure transducers, which had a rated uncertainty of $0.25 \%$ of full scale for all models used. The full-scale range of the seventeen transducers varied from $124.6 \mathrm{~Pa}\left(0.5 " \mathrm{H}_{2} \mathrm{O}\right)$ to $622.9 \mathrm{~Pa}\left(2.5 " \mathrm{H}_{2} \mathrm{O}\right)$, thus the instrument uncertainty varied from $0.31 \mathrm{~Pa}$ to $1.56 \mathrm{~Pa}$, respectively.

The gas density was calculated using the ideal gas law as:

$$
\rho=\frac{P_{s} \cdot M W}{R_{u} \cdot T}
$$

where $P_{s}$ was the measured DT static pressure, $M W$ was the assumed molecular weight of the gases in the DT, $R_{u}$ was the universal gas constant $(8314 \mathrm{~J} /(\mathrm{kmol}-\mathrm{K}))$, and $T$ was the temperature measured at each probe in Kelvin. The molecular weight was assumed equal to the molecular weight of air since the combustion products would typically have a molecular weight similar to that of air ( $27.6-28.3$, compared to 28.97$)$, and would 
typically be diluted with air by a factor of at least 20 . At this high dilution ratio, the molecular weight's contribution to the systematic uncertainty would be negligible (lowest DR of any test was 5 and the percent difference from air was $1.6 \%$; a more typical dilution ratio was 22 which had a percent difference of $0.76 \%$ ) and was thus assumed to be zero. The uncertainty in the temperature measurement using a bare-wire thermocouple was taken as $2.2 \mathrm{~K}$ from the manufacturer. The DT static pressure was calculated by measuring the difference between the ambient pressure, measured by a barometric mercury manometer, and an inclined ethanol manometer connected in a vacuum arrangement to the DT static taps. Calculation of the static pressure is shown in Equation 3.24:

$$
P_{s}=P_{\infty}-\Delta P_{s}=13550 \cdot \mathrm{g} \cdot H_{\text {merc }}-789 \cdot \mathrm{g} \cdot H_{\text {eth }} \cdot \text { ratio }
$$

where $H_{\text {merc }}$ is the height of mercury, $H_{\text {eth }}$ is the height of ethanol, and ratio is the ratio of the inclination for the inclined manometer (typically $1 / 10$ ). The uncertainty in the static pressure was based on the uncertainty of reading the height $\left(B_{H}\right)$ of fluid from the scales, taken as half of the resolution of the scales, or $0.0005 \mathrm{~m}$ for each instrument. Since these are not simple instrument uncertainties, the sensitivity index needs to be calculated for each component, and are given in Equations 3.25 and 3.26 as an example of the calculation procedure.

$$
\begin{gathered}
\theta_{h_{\text {merc }}}=\frac{\partial P_{s}}{\partial H_{\text {merc }}}=13550 \cdot g \\
\theta_{h_{\text {elt }}}=\frac{\partial P_{s}}{\partial H_{\text {eth }}}=-789 \cdot \mathrm{g} \cdot \text { ratio }
\end{gathered}
$$

Once the sensitivity indices were calculated, the combined static pressure systematic uncertainty could be calculated as, 


$$
B_{P_{s}}=\sqrt{\left(\theta_{H_{\text {merc }}} B_{H}\right)^{2}+\left(\theta_{H_{\text {elt }}} B_{H}\right)^{2}}
$$

All the information necessary to calculate the gas density systematic uncertainty was then available as shown in Equation 3.28.

$$
B_{\rho_{i}}=\sqrt{\left(\theta_{P_{s}} B_{P_{s}}\right)^{2}+\left(\theta_{T_{i}} \cdot 2.2\right)^{2}}
$$

where $i$ is the probe of interest. The systematic uncertainty in each velocity measurement could then be found using the differential pressure from the pressure transducer and the density calculated at each Pitot probe as,

$$
B_{u_{i}}=\sqrt{\left(\theta_{P_{d, i}} B_{P_{d, i}}\right)^{2}+\left(\theta_{\rho_{i}} B_{\rho_{i}}\right)^{2}}
$$

Once the uncertainties in the velocities were known, the same process was performed on Equation 3.5 to determine the flow rate systematic uncertainty for each velocity profile, and combined in quadrature by the root sum of the squares method to produce the final total systematic uncertainty in the dilution tunnel flow rate, $B_{Q_{D r}}$.

\subsubsection{Sample Volume}

As discussed in section 3.3.1.1, the total volume sampled was a measure of the sum of the sample flow rates multiplied by the measurement time step (i.e. an integration). Therefore, the uncertainty was a function of the sample flow rate as shown in Equations 3.30-3.36. The sample flow rate (see Figure 3.9 for location) is related to the sample volume as defined previously in Equation 3.8.

$$
\begin{gathered}
Q_{\text {sample }}=\left(\dot{m}_{\text {total }}-\dot{m}_{\text {dilution }}\right) \frac{R_{u} \cdot T_{D T}}{P_{s} \cdot M W} \\
\theta_{T_{D T}}=\left(\dot{m}_{\text {total }}-\dot{m}_{\text {dilution }}\right) \frac{R_{u}}{P_{s} \cdot M W}
\end{gathered}
$$




$$
\begin{aligned}
& \theta_{P_{S}}=-\left(\dot{m}_{\text {total }}-\dot{m}_{\text {dilution }}\right) \frac{R_{u} \cdot T_{D T}}{P_{s}^{2} \cdot M W} \\
& \theta_{\dot{m}_{\text {total }}}=\frac{R_{u} \cdot T_{D T}}{P_{s} \cdot M W} \\
& \theta_{\dot{m}_{\text {lilution }}}=-\frac{R_{u} \cdot T_{D T}}{P_{s} \cdot M W}
\end{aligned}
$$

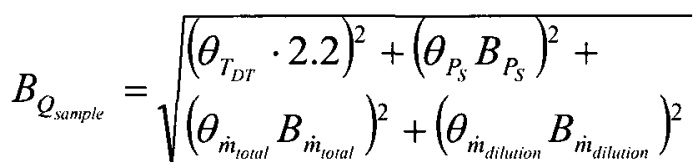

where the systematic uncertainties for the mass flow rates were determined from the reported instrument uncertainties. Since the total volume was calculated as the sum of the individual flow rates, it followed that the uncertainty would be calculated as the sum of the uncertainties as,

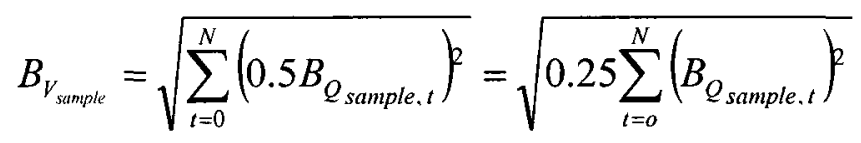

\subsubsection{Total Uncertainty in $Y_{s}$ Measured Gravimetrically}

As mentioned previously, the total uncertainty was a combination of the systematic and precision uncertainties. The final systematic uncertainty was calculated as shown in Equation 3.37. Typical contributions to the total systematic uncertainty are shown in Table 3.9 .

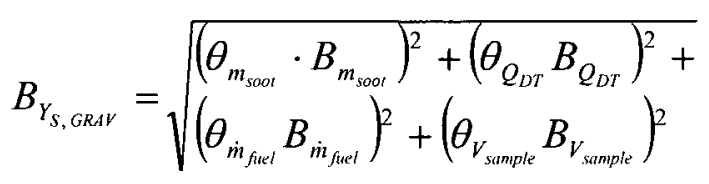


Table 3.9 - Typical contributions to gravimetric systematic uncertainty

\begin{tabular}{|c|c|}
\hline Constituent & Percent of Total Systematic Uncertainty (\%) \\
\hline Mass of soot & 40.387 \\
\hline Dilution tunnel flow rate & 1.318 \\
\hline Total fuel mass flow rate & 23.150 \\
\hline Volume of sample gas & 35.145 \\
\hline
\end{tabular}

For each condition, seven filters with soot were collected, the test conditions recorded (i.e. $Q_{D T}, V_{\text {sample }} \dot{m}_{f u e l}$ ), and the soot yield calculated. The average of these seven tests was taken as the final soot yield and the final systematic uncertainty was calculated based on the systematic uncertainty of each test combined in quadrature as the root sum of the squares multiplied by $\sqrt{1 / N}$. The precision uncertainty, $P$, was calculated using Equation 3.20 where $\sigma$ was the standard deviation of the calculated soot yield, $N=7$ (typically), and the t-value was calculated from the Student's t-distribution table. The final uncertainty in $Y_{s}$ was calculated as shown in Equation 3.38.

$$
U_{Y_{S, G R A}}=\sqrt{\left(B_{Y_{S, G R A V}}\right)^{2}+\left(P_{Y_{S, G R A V}}\right)^{2}}
$$

\subsubsection{Systematic LII Uncertainty}

Examination of Equation 3.13 shows that there are eight components of the soot yield equation for LII analysis. The soot density and LII soot volume fraction uncertainty are presented below with the DT flow rate, temperature, and total fuel mass flow rate uncertainties described above. The uncertainty associated with sampling $\left(\dot{m}_{\text {total }}, \dot{m}_{\text {dilution }}\right)$ was calculated from the reported instrument uncertainties.

\subsubsection{Soot Density}

Since it was outside the scope of this project to measure the soot density directly, the literature was consulted to determine the density of soot, which was revealed to range 
from $1740 \mathrm{~kg} / \mathrm{m}^{3}$ to $2050 \mathrm{~kg} / \mathrm{m}^{3}$ [Choi et al., 1995; Dobbins et al., 1994] as shown in Table 3.10 .

Table 3.10 - Summary of soot density data from literature

\begin{tabular}{|c|c|c|}
\hline Source & Fuel & $\boldsymbol{\rho}_{\text {soot }}\left(\mathbf{k g} / \mathbf{m}^{\mathbf{3}}\right)$ \\
\hline Le Chatelier (1926)* & Acetylene black & 1950 \\
\hline Rossman and Smith (1943)* & Acetylene black & 1840 \\
\hline Janzen (1980)* & Carbon black & 1840 \\
\hline Roessler and Faxvog $(1980)^{*}$ & Acetylene flame & 2050 \\
\hline Nishida and Mukohava (1983)* & Propane flame & 1950 \\
\hline Nelson (1989)* & Acetylene flame & 1900 \\
\hline Choi et al. $[1994]$ & Acetylene/air (premixed) & 1840 \\
\hline Choi et al. $[1995]$ & Acetylene/air (premixed) & 1740 \\
\hline Wu et al. $[1997]$ & Various & 1890 \\
\hline & Mean & $\mathbf{1 8 9 0}$ \\
\hline & $\mathbf{9 5 \%}$ CI & $\mathbf{7 0}$ \\
\hline
\end{tabular}

*Values from data summary given by Dobbins et al. [1994]

The uncertainty on the mass density of soot was estimated from the spread of the values reported in the literature (95\% confidence interval). This did not directly account for systematic uncertainty, but was the best estimate possible of an associated uncertainty. This assumed that the spread in data was considerably larger than the individual systematic errors of the individual measurements. The uncertainty of soot density was conservatively taken as $4 \%$ (or $75.6 \mathrm{~kg} / \mathrm{m}^{3}$ ). It should be noted that the density here refers to the particle density, not the packing density.

\subsubsection{Soot Volume Fraction Measurement Using LII}

Previously, Canteenwalla [2007] had estimated the uncertainty of the instrument to be approximately $6.4 \%$ based on the uncertainty of some optical properties of soot (mainly the absorption function, $\left.E\left(m_{\lambda}\right)\right)$. However, based on experience operating the equipment, this was deemed low. The analysis completed by Canteenwalla did not include any errors introduced during calibration, an error related to a problem of lens chromaticity in the LII emission collection lens, and uncertainties directly related to the measurement 
technique (e.g. centre-wavelength approximation for two-colour calculation of soot particle temperature [Snelling et al., 2005], uncertainty in attenuation values for greyglass optical filters, uncertainty in Photo Multiplier Tube voltages used to measure light intensity, uncertainty in the laser sheet thickness, loss of signal due to dust or soot buildup on test cell optical windows, drift in signal due to changes in laser energy). Considering these factors, a more conservative estimate for uncertainty in the LII measurement of $20 \%$ was adopted.

\subsubsection{Total Uncertainty in $Y_{s}$ Measured Using LII}

The systematic uncertainty in $Y_{s}$ as measured by LII was calculated as,

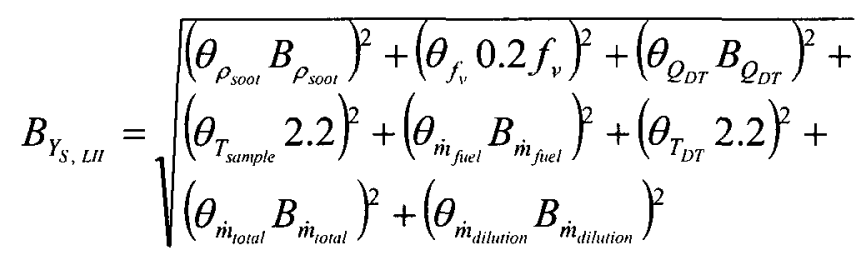

Equation 3.39 represents the instantaneous calculation of $Y_{s}$, and the final value was calculated based on the average of the instantaneous values. A facet of the uncertainty that has not been discussed yet is the inclusion of second order uncertainties, also known as dependent uncertainties. These occur when an uncertainty is calculated where two components of that uncertainty are related. For example, in Equation 3.14, the DT flow rate has an uncertainty associated to the measurement of the temperature in the DT (temperature is related to density, which is related to the velocity, which is related to the total flow rate), but Equation 3.14 has the DT temperature directly in the equation. These uncertainties are not independent of each other, as is typically assumed in Equation 3.18 when combining systematic uncertainties. However, the value is so small in comparison 
to the other contributors of uncertainty $(<0.0001 \%$ of total systematic uncertainty, see Table 3.11) that it could be ignored.

Table 3.11 - Typical contributions to LII systematic uncertainty

\begin{tabular}{|c|c|}
\hline Constituent & Percent of Total Systematic Uncertainty (\%) \\
\hline Soot density & 3.801 \\
\hline $\begin{array}{c}\text { Soot volume fraction } \\
\text { measurement using LII }\end{array}$ & 95.253 \\
\hline Dilution tunnel flow rate & 0.014 \\
\hline Sample temperature & 0.099 \\
\hline Fuel mass flow rate & 0.533 \\
\hline Dilution tunnel temperature & 0.042 \\
\hline Total sample flow rate & 0.001 \\
\hline Dilution air flow rate & 0.257 \\
\hline Dependent uncertainty & $<0.0001$ \\
\hline
\end{tabular}

The precision uncertainty, $P$, was calculated using Equation 3.20 where $\sigma$ is the standard deviation of the calculated soot yield, $N=600$ (for a test of $\sim 5 \mathrm{~min}$ ), and the $\mathrm{t}$-value was obtained from the Student's t-distribution table. The final uncertainty in $Y_{s}$ was then calculated as shown in Equation 3.40.

$$
U_{Y_{S, L I I}}=\sqrt{\left(B_{Y_{S, L I}}\right)^{2}+\left(P_{Y_{S, L I I}}\right)^{2}}
$$

Results of the uncertainty analysis will be shown in the next Chapter as the error bars on all graphs. 


\section{Chapter 4}

\section{Results}

Soot yield measurements were completed for a wide range of operating conditions as presented in the following sections. Gravimetric and LII techniques were used to measure the soot yield to improve the confidence in the results obtained compared to the work of Canteenwalla [2007], where only the LII technique was used. The soot yield results as measured by the two techniques are presented side by side in Section 4.1. Measured visible flame length data are presented in Section 4.2.

\subsection{Soot Yield Results}

Soot yields from turbulent diffusion flames were measured for a variety of conditions. Six different fuel mixtures (light (L), average (AVG), and heavy (H) 6- and 4-component mixtures) and one pure fuel (methane) were used with burner tip inner diameters of 12.7, 25.4, 38.1, 50.8, and $76.2 \mathrm{~mm}$. For the AVG-6-component mixture, results were collected at all attainable diameter and exit velocity combinations, limited by the ranges of available flow controllers. For the remainder of the mixtures, a representative set of conditions were taken mainly on the $25.4 \mathrm{~mm}$ burner for all exit velocities and at an exit velocity of $0.5 \mathrm{~m} / \mathrm{s}$ for all burner diameters. The $12.7 \mathrm{~mm}$ burner conditions were 
extended to include exit velocities of $3.6,5$, and $7.5 \mathrm{~m} / \mathrm{s}$. A summary of the test conditions is given in Table 4.1, and a full summary of measured results and parameters are given in Appendix D.

Table 4.1 - Summary of fuel mixtures used at each test condition

\begin{tabular}{|c|c|c|c|c|c|c|}
\hline \multirow{2}{*}{$\begin{array}{l}\text { I.D. } \\
\text { (mm) }\end{array}$} & \multicolumn{6}{|c|}{ Set Point Exit Velocity } \\
\hline & $0.1 \mathrm{~m} / \mathrm{s}$ & $0.25 \mathrm{~m} / \mathrm{s}$ & $0.5 \mathrm{~m} / \mathrm{s}$ & $0.9 \mathrm{~m} / \mathrm{s}$ & $1.5 \mathrm{~m} / \mathrm{s}$ & $2.2 \mathrm{~m} / \mathrm{s}$ \\
\hline 12.7 & - & - & $\begin{array}{c}\text { AVG-6-Mix } \\
\text { H-6-Mix }\end{array}$ & AVG-6-Mix & AVG-6-Mix & AVG-6-Mix \\
\hline 25.4 & $\begin{array}{c}\text { AVG-6-Mix } \\
\text { H-6-Mix } \\
\text { AVG-4-Mix } \\
\text { H-4-Mix } \\
\text { Methane }\end{array}$ & $\begin{array}{c}\text { AVG-6-Mix } \\
\text { L-6-Mix } \\
\text { H-6-Mix } \\
\text { AVG-4-Mix } \\
\text { L-4-Mix } \\
\text { H-4-Mix } \\
\text { Methane }\end{array}$ & $\begin{array}{c}\text { AVG-6-Mix } \\
\text { L-6-Mix } \\
\text { H-6-Mix } \\
\text { AVG-4-Mix } \\
\text { L-4-Mix } \\
\text { H-4-Mix } \\
\text { Methane } \\
\end{array}$ & $\begin{array}{c}\text { AVG-6-Mix } \\
\text { L-6-Mix } \\
\text { H-6-Mix } \\
\text { AVG-4-Mix } \\
\text { L-4-Mix } \\
\text { H-4-Mix } \\
\text { Methane }\end{array}$ & $\begin{array}{c}\text { AVG-6-Mix } \\
\text { L-6-Mix } \\
\text { H-6-Mix } \\
\text { AVG-4-Mix } \\
\text { L-4-Mix } \\
\text { H-4-Mix } \\
\text { Methane }\end{array}$ & $\begin{array}{c}\text { AVG-6-Mix } \\
\text { L-6-Mix } \\
\text { H-6-Mix } \\
\text { AVG-4-Mix } \\
\text { L-4-Mix } \\
\text { H-4-Mix } \\
\text { Methane }\end{array}$ \\
\hline 38.1 & AVG-6-Mix & AVG-6-Mix & $\begin{array}{c}\text { AVG-6-Mix } \\
\text { L-6-Mix } \\
\text { H-6-Mix }\end{array}$ & AVG-6-Mix & - & - \\
\hline 50.8 & $\begin{array}{l}\text { AVG-6-Mix } \\
\text { AVG-4-Mix }\end{array}$ & $\begin{array}{c}\text { AVG-6-Mix } \\
\text { AVG-4-Mix } \\
\text { L-4-Mix } \\
\text { H-4-Mix }\end{array}$ & $\begin{array}{c}\text { AVG-6-Mix } \\
\text { L-6-Mix } \\
\text { H-6-Mix } \\
\text { AVG-4-Mix } \\
\text { L-4-Mix } \\
\text { H-4-Mix }\end{array}$ & - & - & - \\
\hline 76.2 & $\begin{array}{l}\text { AVG-6-Mix } \\
\text { AVG-4-Mix }\end{array}$ & $\begin{array}{c}\text { AVG-6-Mix } \\
\text { AVG-4-Mix } \\
\text { L-4-Mix } \\
\text { H-4-Mix } \\
\end{array}$ & - & - & - & - \\
\hline
\end{tabular}

Measured soot yields for the $25.4 \mathrm{~mm}$ burner fuelled with the AVG-6-component mixture are shown in Figure 4.1 for both the gravimetric and LII-based experiments. The two techniques generally show excellent agreement within the bounds of uncertainty. As the mass flow rate was increased, the soot yield increased to a maximum value before starting to decline as the mass flow rate was increased further. This implies the existence of an "optimum" soot generating condition, perhaps corresponding to a flame regime transition, or to a balance between competing effects of soot growth and oxidation through the flame. Figure 4.2 shows the soot yield results for all burner diameters burning the average 6-component mixture. The $12.7 \mathrm{~mm}$ burner exhibits a similar up- 
down trend as the $25.4 \mathrm{~mm}$ burner when the mass flow rate is increased, although at a reduced scale. By contrast, the larger burners $(38.1,50.8,76.2 \mathrm{~mm})$ do not exhibit the decreasing trend that was observed with the $25.4 \mathrm{~mm}$ burner, demonstrating that mass flow rate may not be an optimal scaling parameter, and/or that the different sized burners are operating in different regimes. Although the magnitudes of soot yield vary, the largest three burners all exhibit a similar trend of an initial increase in soot yield as mass flow rate is increased, followed by a levelling out of the soot yield. The error bars in all figures represent the uncertainty determined by the methods described in Chapter 3 (i.e. $\left.Y_{s} \pm U_{Y S}\right)$

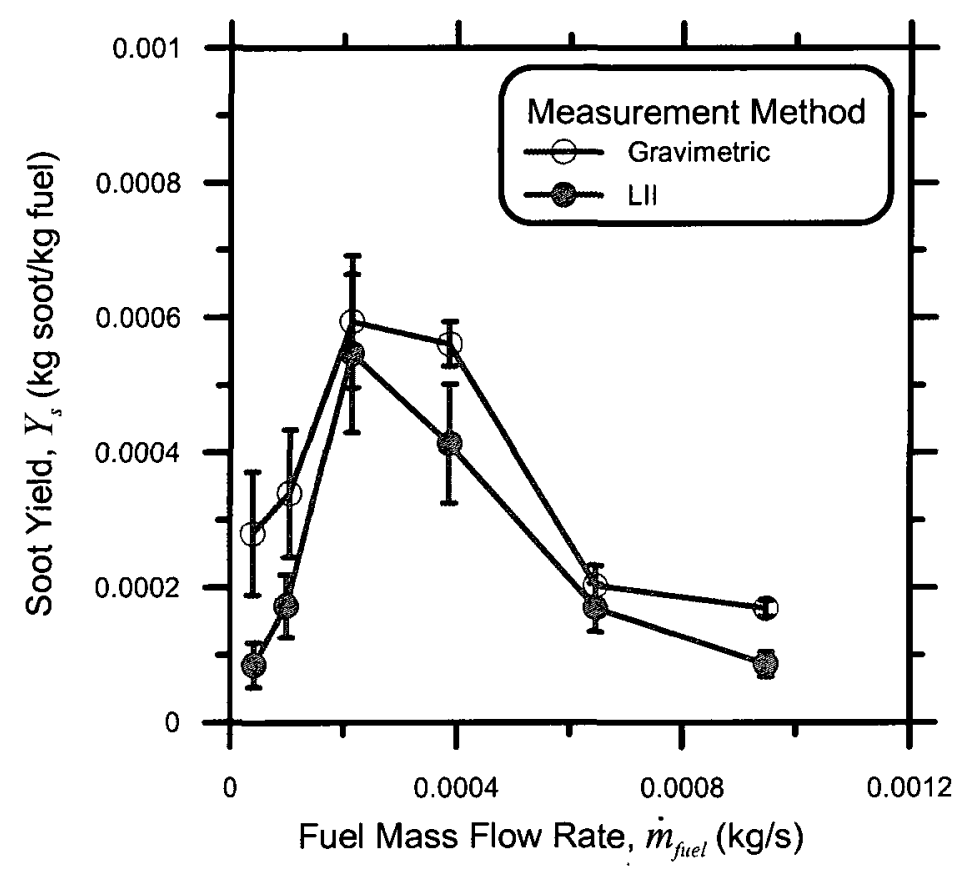

Figure 4.1 - Soot yield from the $25.4 \mathrm{~mm}$ burner burning the AVG-6-component mixture measured using both the gravimetric technique and the LII method 


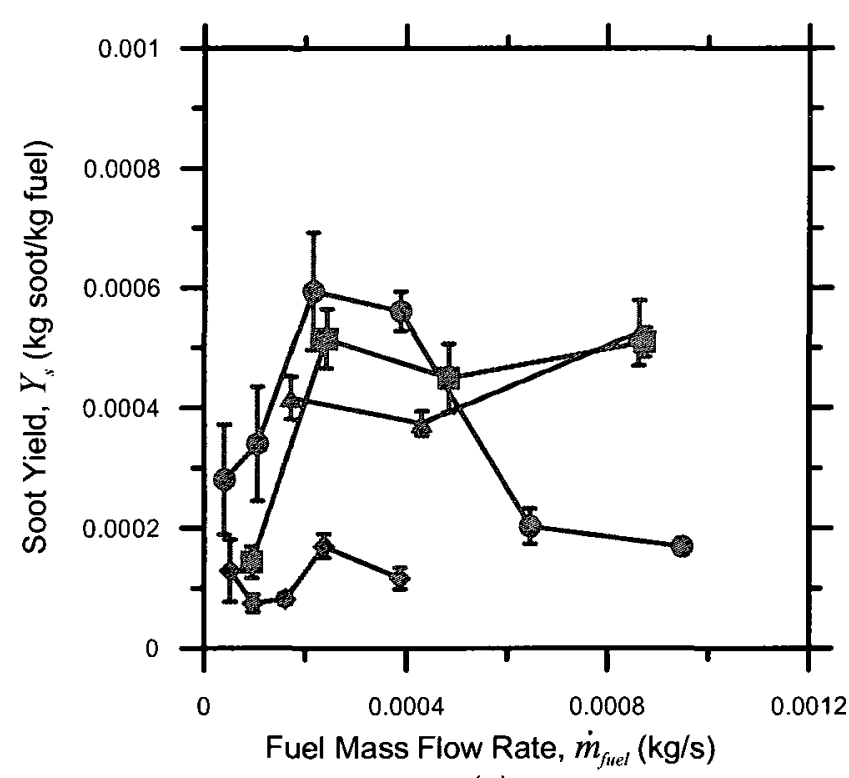

(a)

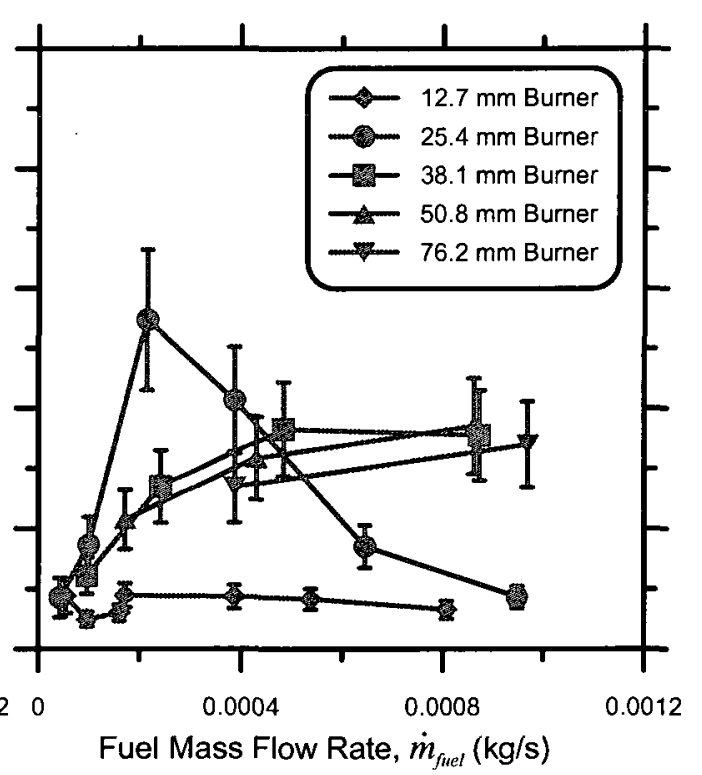

(b)

Figure 4.2 - Soot yield for a range of burner diameters burning the AVG-6-component mixture measured using (a) the gravimetric technique and (b) the LII method

Figure 4.3 shows the soot yield for various burner diameters where the average exit velocity was fixed among the different burners. All data shown were for combustion of the AVG-6-component fuel mixture. If Figure 4.3 (a) is considered, no obvious trends exist, although it is clear that the $12.7 \mathrm{~mm}$ diameter burner nets considerably lower soot yields for all exit velocities as was noted above. The scatter of the curves does not allow any further information to be inferred. If Figure 4.3 (b) is considered, the LII data set shows somewhat more consistent trends than the gravimetric data. As in Figure 4.3 (a), the $12.7 \mathrm{~mm}$ burner diameter produces universally low soot yields at all velocities. However, data for the lowest two velocities show a gradual rise in soot yield with increasing diameter whereas the middle two velocities show a peak soot yield followed by a settling at reduced values as diameter is increased. Because of facility limits, there is not enough data for the exit velocities of 1.5 and $2.2 \mathrm{~m} / \mathrm{s}$ to make any kind of suggestions; although curiously, they do line up with the curves of 0.25 and $0.1 \mathrm{~m} / \mathrm{s}$, respectively. 


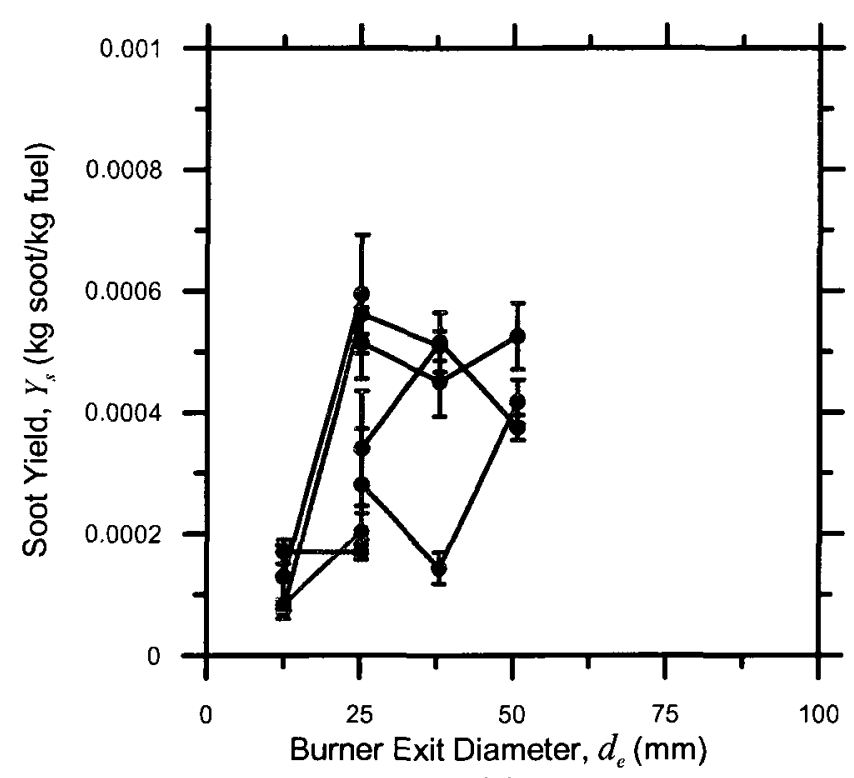

(a)

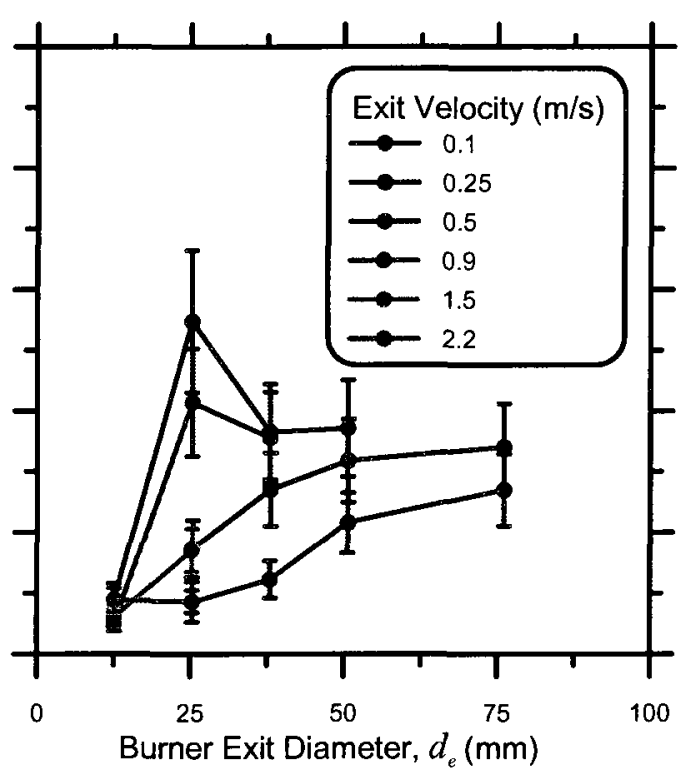

(b)

Figure 4.3 - Soot yield values for constant set point exit velocities for a range of burner diameters burning the AVG-6-component mixture measured using (a) the gravimetric technique and (b) the LII method

Soot yield data for the different fuel mixtures are compared in Figure 4.4 for the $25.4 \mathrm{~mm}$ burner. All seven fuel compositions exhibit similar increasing/decreasing trends with mass flow rate as shown previously. The soot yield varies with fuel composition as expected, i.e. the heavy fuel mixture has the highest sooting propensity, the light fuel mixture has the lowest, and the 4-component undiluted fuel mixtures have a higher sooting propensity than the equivalent 6-component mixtures. This shows the importance of fuel chemistry on the soot yield. For the same set point exit velocity $(0.5 \mathrm{~m} / \mathrm{s})$, the soot yield changes from 0.00044 to $0.00079 \mathrm{~kg} \mathrm{soot} / \mathrm{kg}$ fuel for the 6component light and heavy mixtures measured gravimetrically (an increase of $\sim 80 \%$ ), even though the methane fraction in the fuel mixtures decreases by only $18 \%(91.14 \%$ in the light mixture vs. $74.54 \%$ in the heavy mixture). Even more dramatically, at the same exit velocity $(0.5 \mathrm{~m} / \mathrm{s})$, the soot yield changes from $\sim 0.000066$ to $\sim 0.00034 \mathrm{~kg}$-soot $/ \mathrm{kg}$ fuel as the fuel is switched from pure methane to the L-6-component mixtures $(91.14 \%$ 
methane) as measured by the LII technique (an increase of $\sim 415 \%$ ), while the percent of methane drops by only $\sim 9 \%$. Clearly, these results show that even for a similar range of fuel mixtures typical of the range found in flare gas samples, the effects of chemistry can be quite significant.

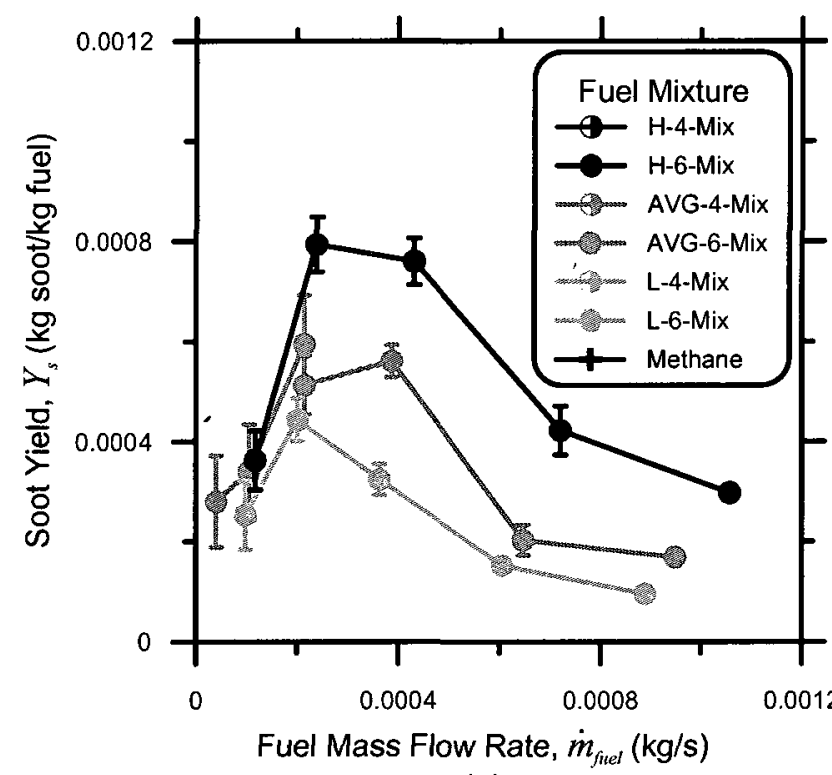

(a)

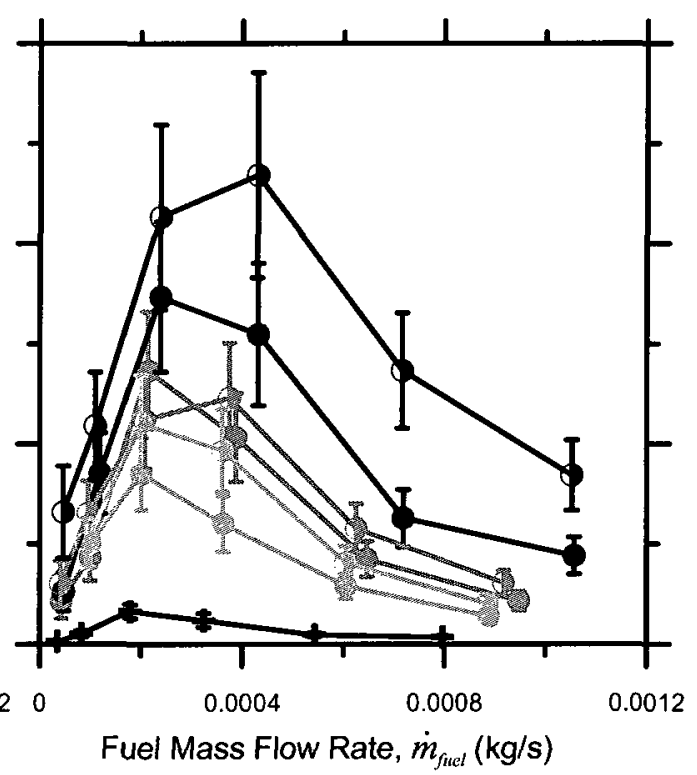

(b)

Figure 4.4 - Soot yield values from the $25.4 \mathrm{~mm}$ burner burning the L-, AVG-, and H- 6- and 4component mixtures measured using (a) the gravimetric technique and (b) the LII method

Figure 4.5 shows the variation of soot yield with burner diameter for a constant exit velocity of $0.5 \mathrm{~m} / \mathrm{s}$ for the six fuel mixtures measured gravimetrically (Figure 4.5 (a)) and by the LII technique (Figure 4.5 (b)). The only discernable trends in Figure 4.5 (a) are the previously mentioned inverse scaling of soot yield with the methane content in the fuel, and lower soot yield values obtained on the $12.7 \mathrm{~mm}$ burner. Measured soot yields increase as the burner diameter is increased; results for larger diameters remain either stable or decrease slightly. Due to restrictions on the maximum flow rate attainable, data were not collected for the $76.2 \mathrm{~mm}$ burner at an exit velocity of $0.5 \mathrm{~m} / \mathrm{s}$. 


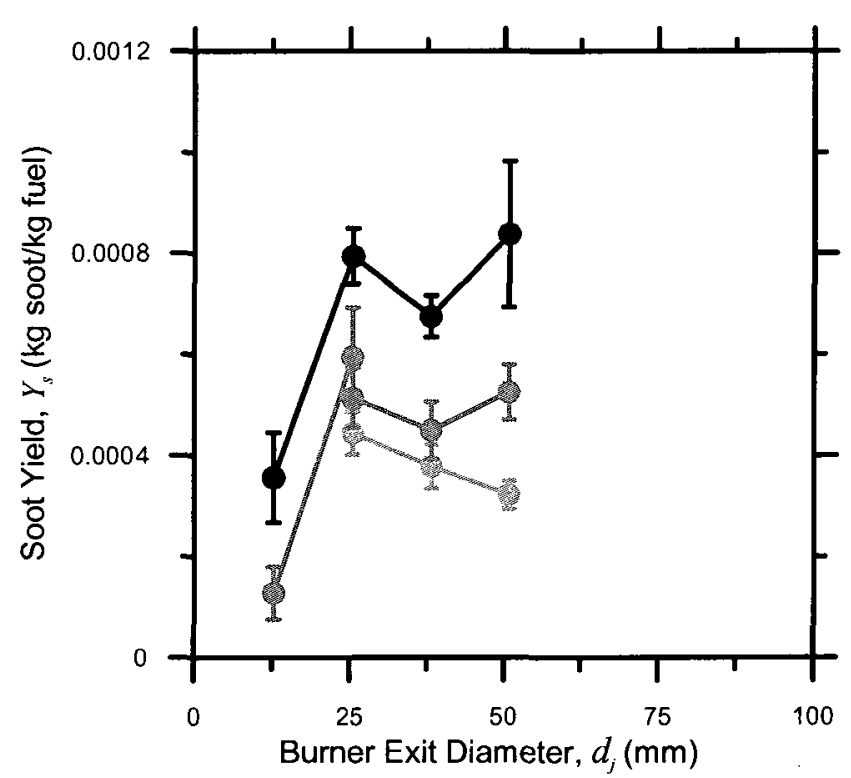

(a)

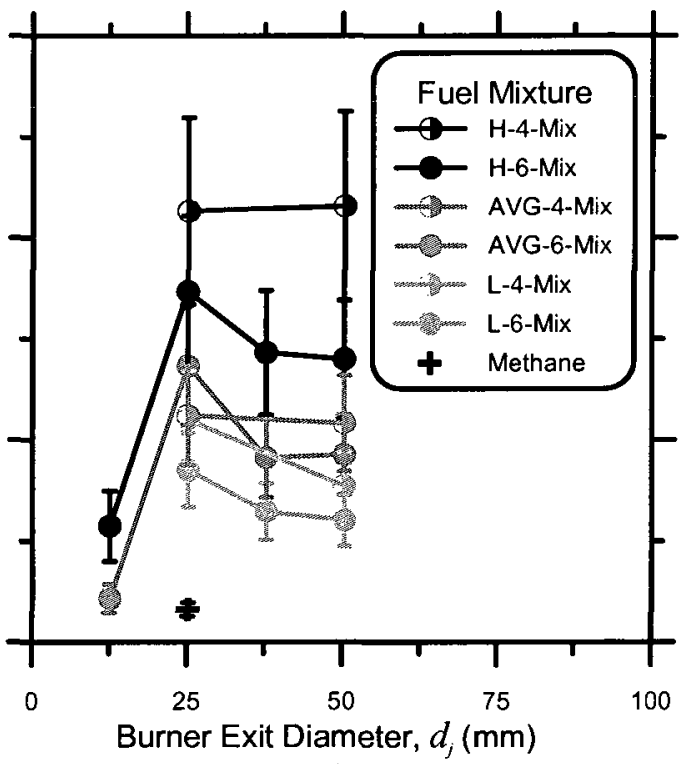

(b)

Figure 4.5 - Soot yield at a constant set point exit velocity of $0.5 \mathrm{~m} / \mathrm{s}$ burning the L-, AVG-, and H- 6and 4-component mixtures measured using (a) the gravimetric technique and (b) the LII method

Figure 4.6 shows the varying soot yield with burner diameter for a constant exit velocity of $0.25 \mathrm{~m} / \mathrm{s}$ for the AVG-6-component fuel mixture, L-, AVG-, and H- 4component fuel mixtures, and the methane data point for reference. All four mixtures exhibit the same rising and settling trend with diameter, but more data with larger burners would be required to definitively state that the soot yield would not subsequently rise or fall. These two plots (Figure 4.5 and Figure 4.6) show that while there is some consistency in the observed soot yield behaviour among the different test conditions, neither exit velocity or burner diameter alone are sufficient scaling parameters to correlate the data. 


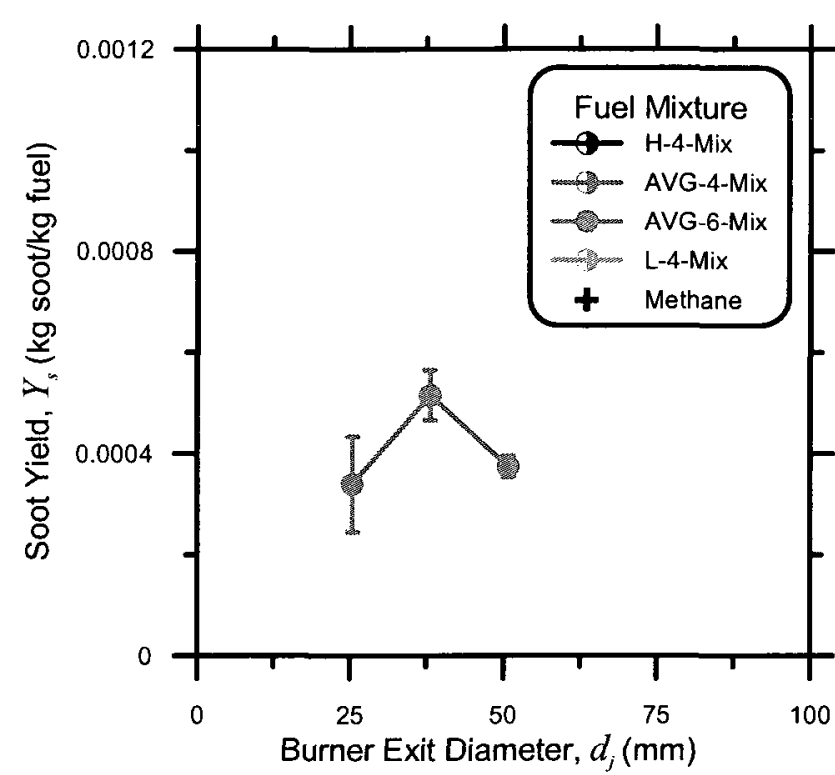

(a)

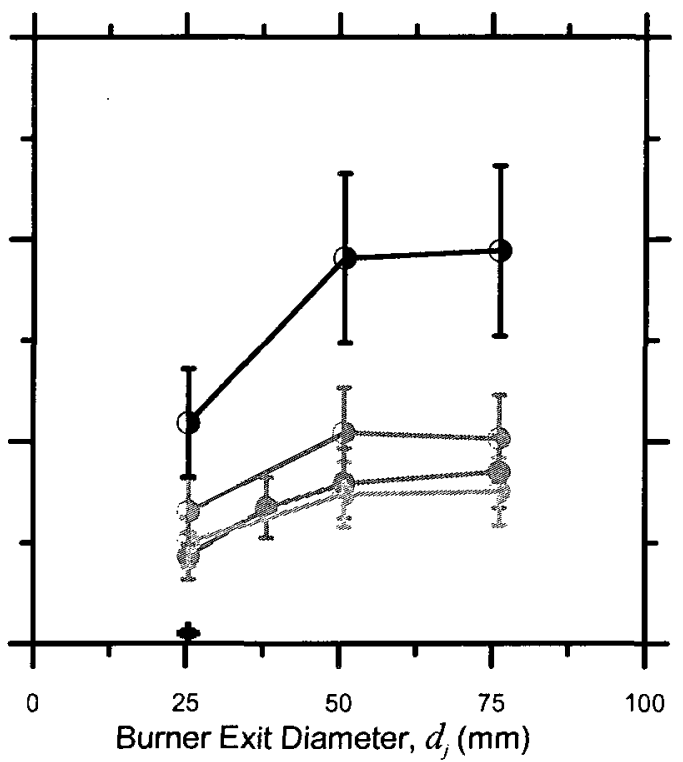

(b)

Figure 4.6 - Soot yield at a constant set point exit velocity of $0.25 \mathrm{~m} / \mathrm{s}$ burning the L-, AVG-, and H4-component mixture and the average 6-component mixture measured using (a) the gravimetric technique and (b) the LII method

\subsubsection{Comparison of Gravimetric and LII}

The primary purpose of employing the gravimetric technique was to gain confidence in the values reported by the LII technique, since LII is a much less time-intensive measurement method. Figure 4.7 shows the comparison between measured soot yield data for similar conditions. The solid line indicates a reference 1:1 correspondence. Data derived from LII measurements consistently shows lower soot yield magnitudes than data obtained with the gravimetric measurement method. This was anticipated as mentioned previously in section 3.3 .2 , since the LII technique will evaporate or sublimate other volatile organics bound up with the soot aggregates that the gravimetric technique will not, resulting in higher total masses being obtained with the gravimetric measurements. In this sense, the LII recorded values can be thought of as representing only the elemental carbon (EC) portion of the soot, whereas the gravimetric values represent the total carbon (TC - EC plus organic carbon, OC). The correspondence between the two techniques is 
sufficiently close that the soot generated from the LSF appears to be predominantly EC. Based on the slope of the best-fit line, it could be deduced that the EC fraction is approximately $80 \%$ of the TC, giving a $4: 1$ ratio of EC to $\mathrm{OC}$. This value is close to the ratio of 4.75:1 reported by Bond et al. [2006] for particles smaller than $2.5 \mu \mathrm{m}$ produced from an oil-burning boiler. Since the EC is of particular interest in this work, and since the difference between the techniques is close to negligible within experimental error, only the LII recorded measurements will be discussed henceforth, but the agreement of these techniques gives an important confidence in the measured results.

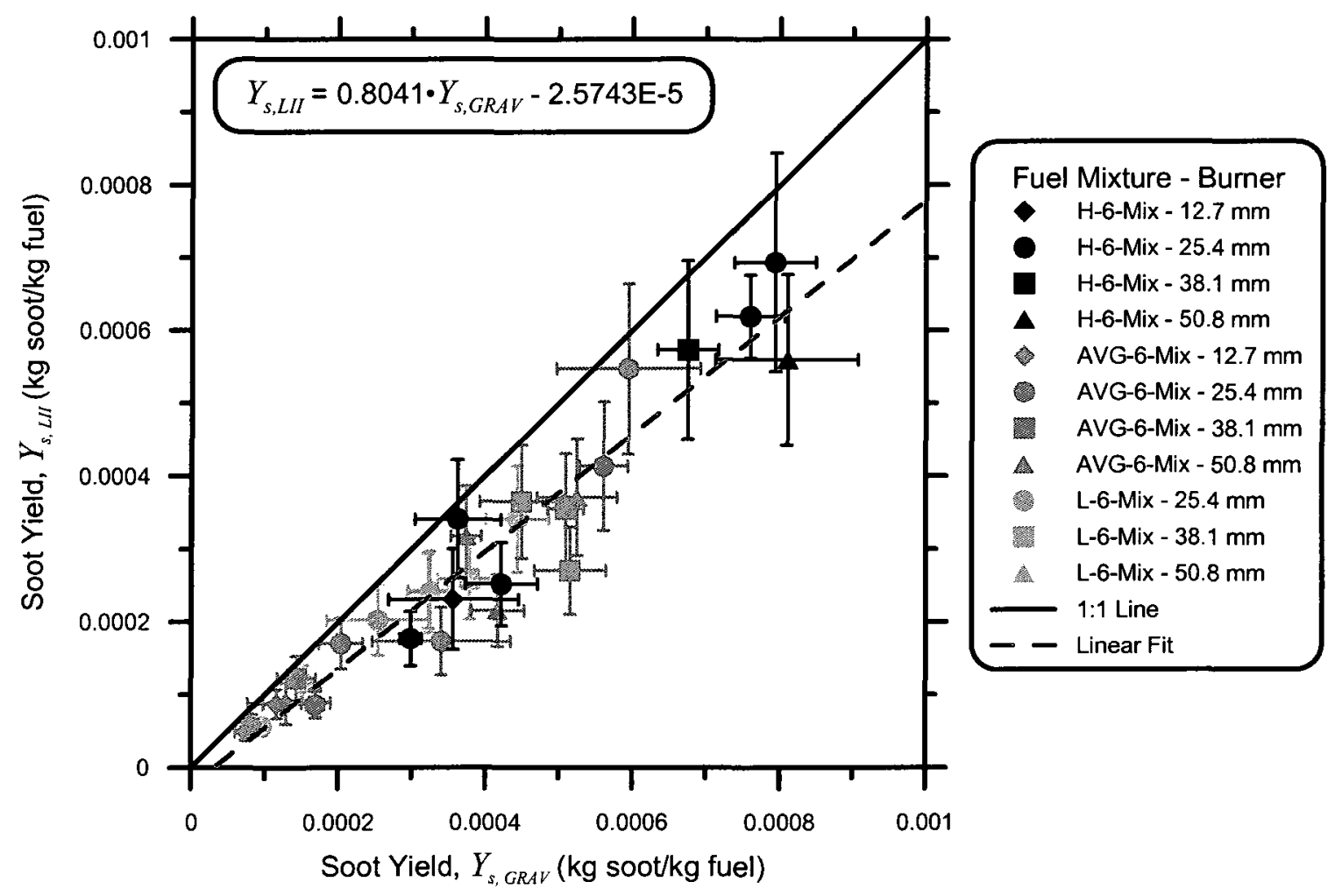

Figure 4.7 - Comparison of soot yield measured using the LII and gravimetric techniques

\subsection{Flame Length Results}

Measured visible flame length data are shown in Figure 4.8. Flame length was measured as a potential parameter used for scaling soot emission. The graphs show that for all 
fuels, burner diameters, and flow rates, the data follows a steady increase in flame length with increasing mass flow rate, as was expected. According to conventional diffusion flame models, this demonstrates that for all of the conditions studied, the flames can be classed as buoyancy-dominated. The measured visible flames were compared to the estimated flame length calculated using the method proposed by Delichatsios [1993a] as shown in Figure 4.9. This graph shows that there is good agreement between the measured and calculated values.

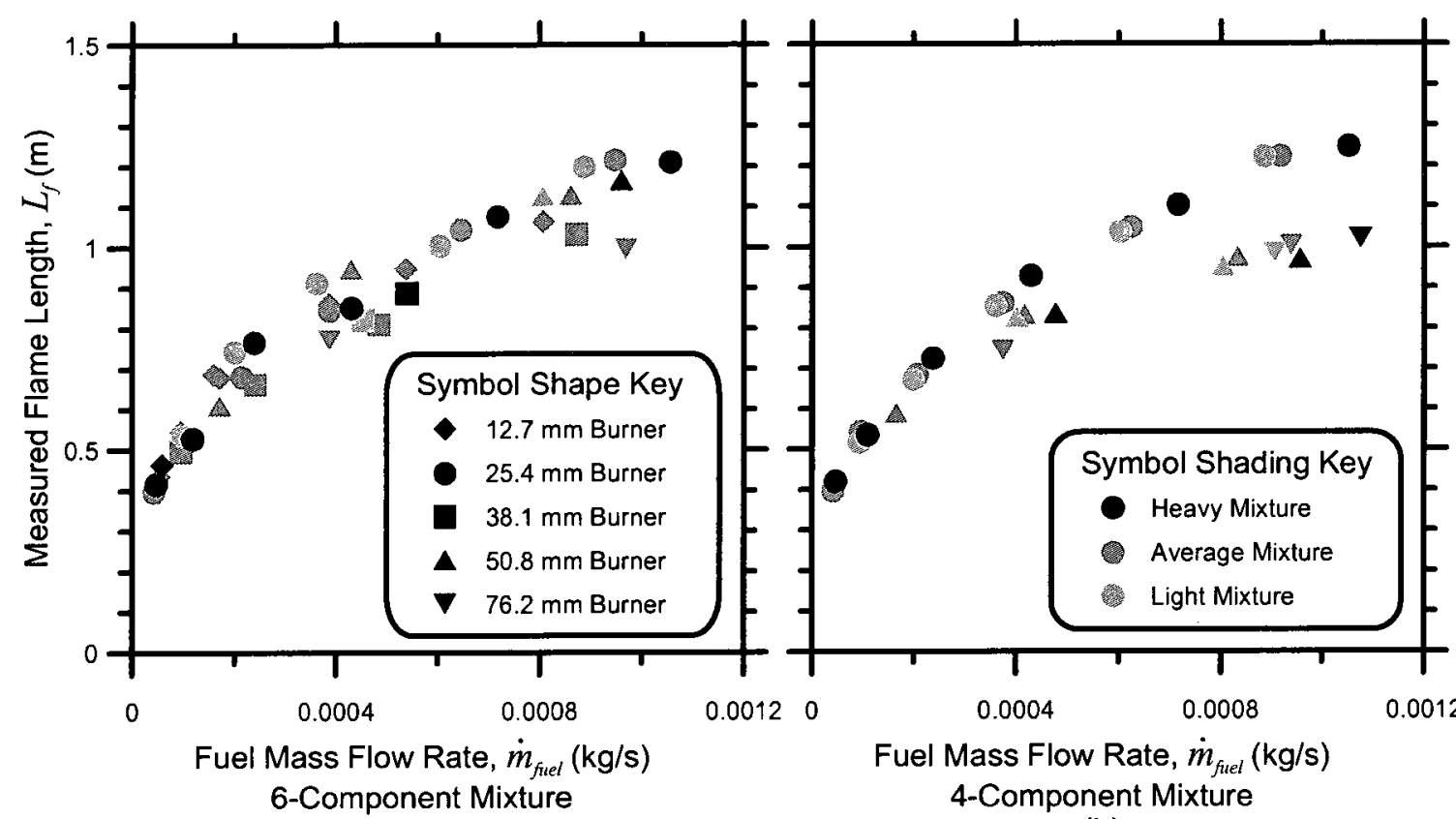

(a)

(b)

Figure 4.8 - Measured visible flame lengths for all burner diameters and flow rates burning L-, AVG-, and H- (a) 6-component mixtures and (b) 4-component mixtures 


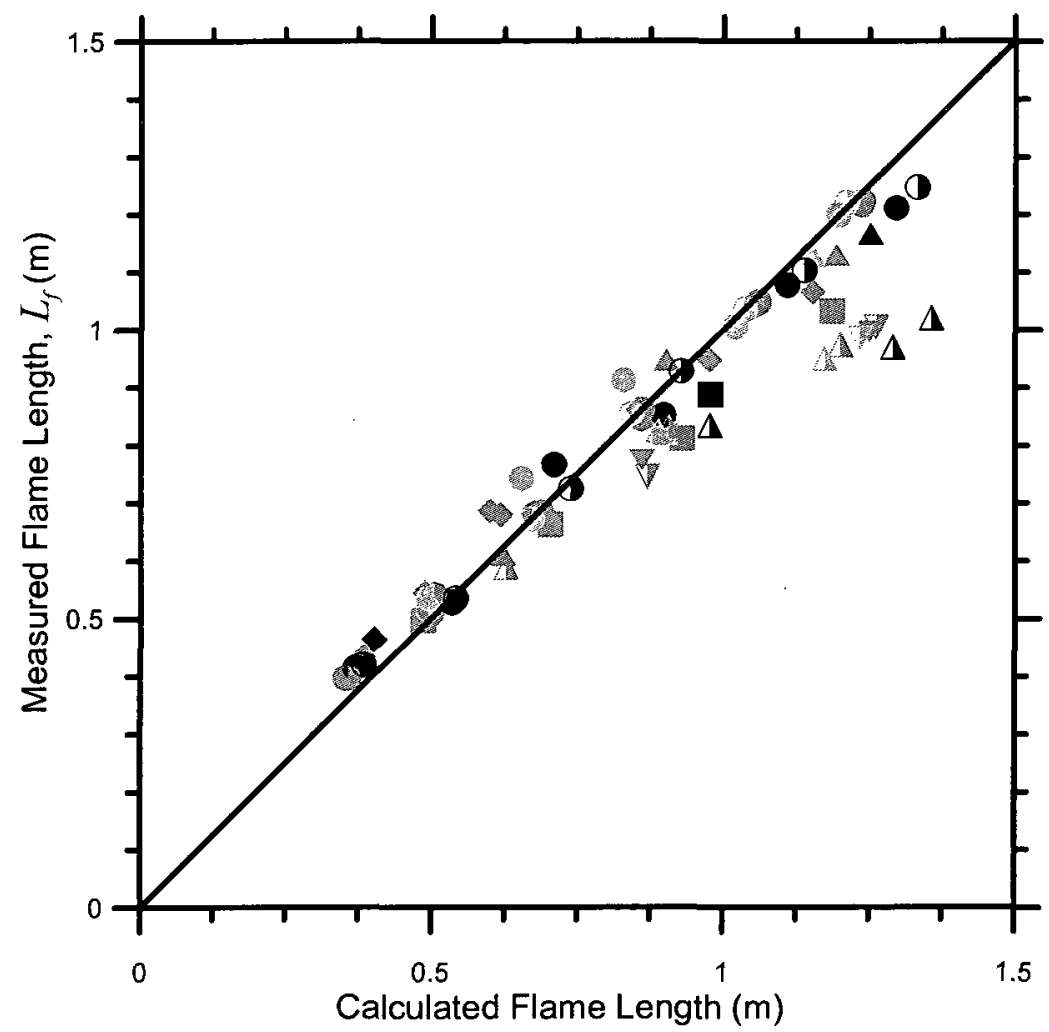

\footnotetext{
Fuel Mixture - Burner

- AVG-6-Mix - $12.7 \mathrm{~mm}$

2. AVG-6-Mix $-25.4 \mathrm{~mm}$

解 AVG-6-Mix $-38.1 \mathrm{~mm}$

A AVG-6-Mix $-50.8 \mathrm{~mm}$

AVG-6-Mix $-76.2 \mathrm{~mm}$

稳 L-6-Mix $-25.4 \mathrm{~mm}$

2. L-6-Mix - $38.1 \mathrm{~mm}$

. L-6-Mix $-50.8 \mathrm{~mm}$

- H-6-Mix - $12.7 \mathrm{~mm}$

- H-6-Mix - $25.4 \mathrm{~mm}$

D $H-6-M i x-38.1 \mathrm{~mm}$

A H-6-Mix - $50.8 \mathrm{~mm}$

AVG-4-Mix $-25.4 \mathrm{~mm}$

AVG-4-Mix $-50.8 \mathrm{~mm}$

$\checkmark \quad$ AVG-4-Mix $-76.2 \mathrm{~mm}$

1) L4-Mix - $25.4 \mathrm{~mm}$

1) L-4-Mix $-50.8 \mathrm{~mm}$

L-4-Mix $-76.2 \mathrm{~mm}$

(1) H-4-Mix - $25.4 \mathrm{~mm}$

$\Delta \quad$ H-4-Mix $-50.8 \mathrm{~mm}$

$\Delta \quad H-4-M i x-76.2 \mathrm{~mm}$
}

Figure 4.9 - Comparison of measured visible flame length and calculated flame length

It has been shown in the current chapter that there is good agreement between the two measurement techniques. The lack of a simple characteristic (i.e. burner diameter, exit velocity, or mass flow rate) to scale the measured soot emissions has led to the discussion of Chapter 5, which will consider several parameters for scaling the measured soot emissions based on aerodynamic properties and fuel chemistry effects. 


\section{Chapter 5}

\section{Analysis and Discussion}

The analysis and discussion presented in this chapter are based on the LII measurement results. The LII measurements were used because they covered a larger range of test conditions, and it has been shown in Chapter 4 that there is good agreement between the gravimetrically-measured and the LII-measured soot yield.

\subsection{Comparisons with Literature}

The work of Canteenwalla [2007] was the predecessor to the current project. As a first step in analyzing the present results, a comparison was made to those of Canteenwalla [2007]. Figure 5.1 includes current experimental results and those of Canteenwalla [2007] for methane burned in the $25.4 \mathrm{~mm}$ lab-scale flare. Overall, the results follow the same trend, but at mass flow rates less than $\sim 0.0005 \mathrm{~kg} / \mathrm{s}$ soot yields measured in the present work are lower than those measured by Canteenwalla [2007]. 


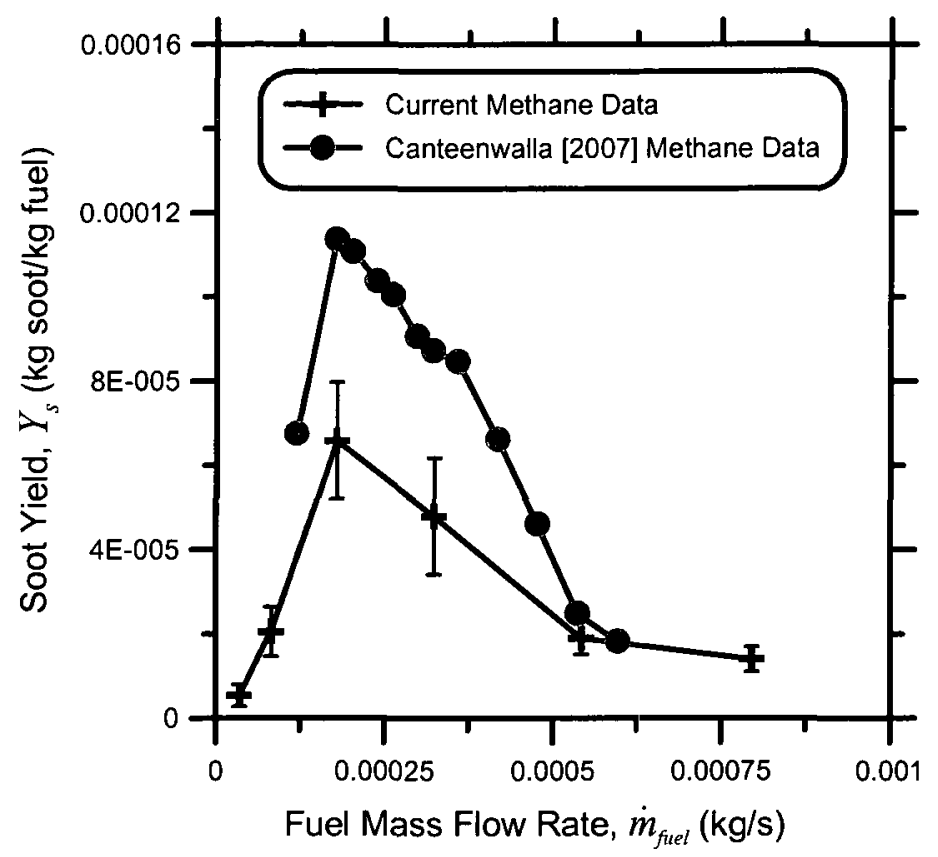

Figure 5.1 - Comparison of current experimental results with Canteenwalla [2007] for the $25.4 \mathrm{~mm}$ burner burning methane

After extensive measurements and data analysis, Canteenwalla [2007] observed large uncertainties in his data that were ultimately attributed to dilution ratio effects. Additionally, the LII apparatus used in his work was subsequently found to have technical problems with the emission collection optics, which added an unknown error to the results. Thus, the original error bars for the data of Canteenwalla [2007] were omitted from Figure 5.1. These two observations were the primary impetus for the development of a new sampling protocol in the current work, and for the incorporation of the gravimetric sampling system into the current experiment to provide a comparison for the LII results. With the adoption of achromatic optics in the LII200 apparatus, good agreement was observed between LII and gravimetric measurements.

To the author's knowledge, the only other data for diffusion flames burning similar fuel compositions that could be used for comparison with the present data were those of Becker and Liang [1982], who reported soot generation efficiency $(S G E)$ data for vertical 
diffusion flames burning methane. Figure 5.2 shows a comparison of $S G E$ data for methane flames from Becker and Liang [1982] and corresponding results from the current work. The data of Becker and Liang [1982] were obtained by digitizing the plot in their published paper in which they reported soot yields in terms of the $S G E$. The values measured by Becker and Liang [1982] were approximately an order of magnitude larger than values obtained in the current work. This discrepancy is most likely due to the different flow conditions. As mentioned in Chapter 2, the work of Becker and Liang [1982] was completed with much smaller burner diameters $(2.5-10.9 \mathrm{~mm})$ and much higher exit velocities $(13-75 \mathrm{~m} / \mathrm{s})$ than the current work. Considering the variation of soot yield (and $S G E$ ) at different flow conditions shown in the previous chapter, these differences could simply be a reflection of different flame regimes. Furthermore, given the difficulties in measuring soot yields accurately, it is also possible that the disparity is at least partially attributable to differences in sampling protocols. Unfortunately, Becker and Liang [1982] only provided limited detail about their soot sampling system, simply stating that it was filter-based, and did not show an uncertainty analysis. Finally, the abscissa of the plot in Figure 5.2, $R i_{L}$, has a power of three dependence on the measured flame length, so that even small discrepancies in flame length measurement would have a large impact on $R i_{L}$. 


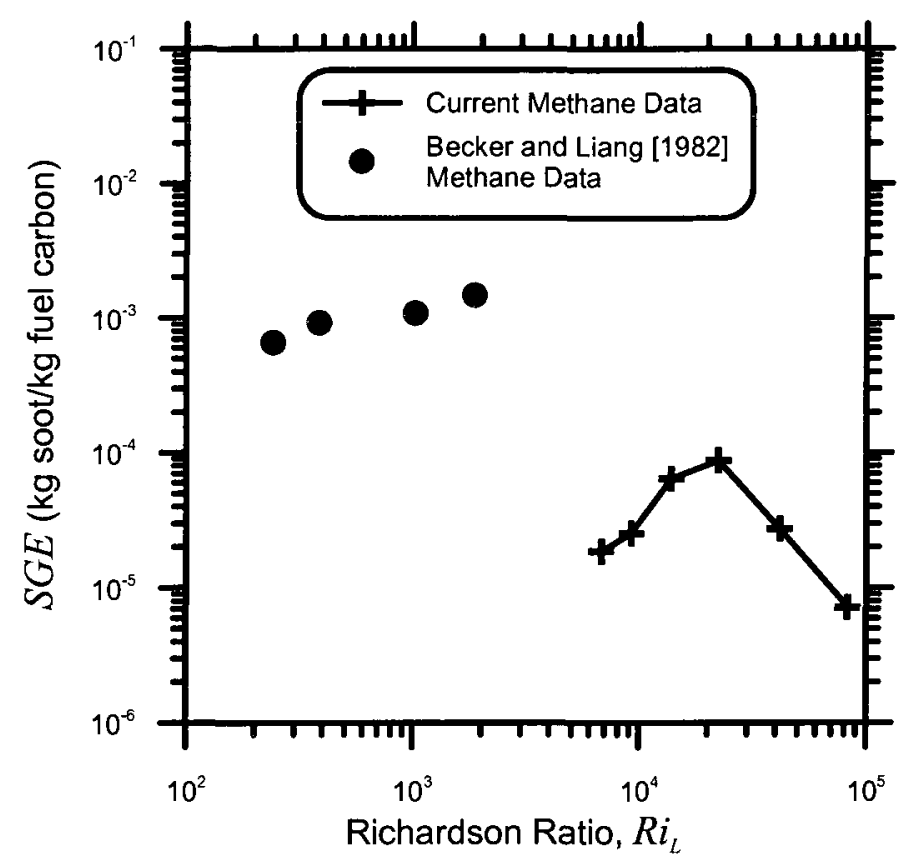

Figure 5.2 - Comparison of measured $S G E$ values for methane with methane data of Becker and Liang [1982]. $R i_{L}=g L_{f}^{3}\left(u_{e} d_{e}\right)^{-2} \rho_{\infty} / \rho_{e}$

From the published data available in the work of Becker and Liang [1982], it was not possible to directly calculate values of the global Froude number required to plot their points on the regime map suggested by Delichatsios [1993a]. However, recognizing that the Froude number is related to the square root of the inverse of the Richardson ratio, it was possible to estimate a conversion based on results of the current data. As shown in Figure 5.3, by calculating both the Richardson ratio and global Froude number at identical conditions in the current data, a simple conversion equation was developed. Using this relationship and a range of Reynolds numbers calculated from the original data of Becker and Liang [1982], their range of conditions could be estimated and plotted as a dashed box on the regime diagram shown in Figure 5.4. This graph shows that the methane data reported by Becker and Liang [1982] correspond to the turbulentbuoyant/transition-shear and turbulent-momentum regimes, which differ from the present 
data. Therefore, differences in magnitudes of soot generation efficiency are not necessarily unexpected.

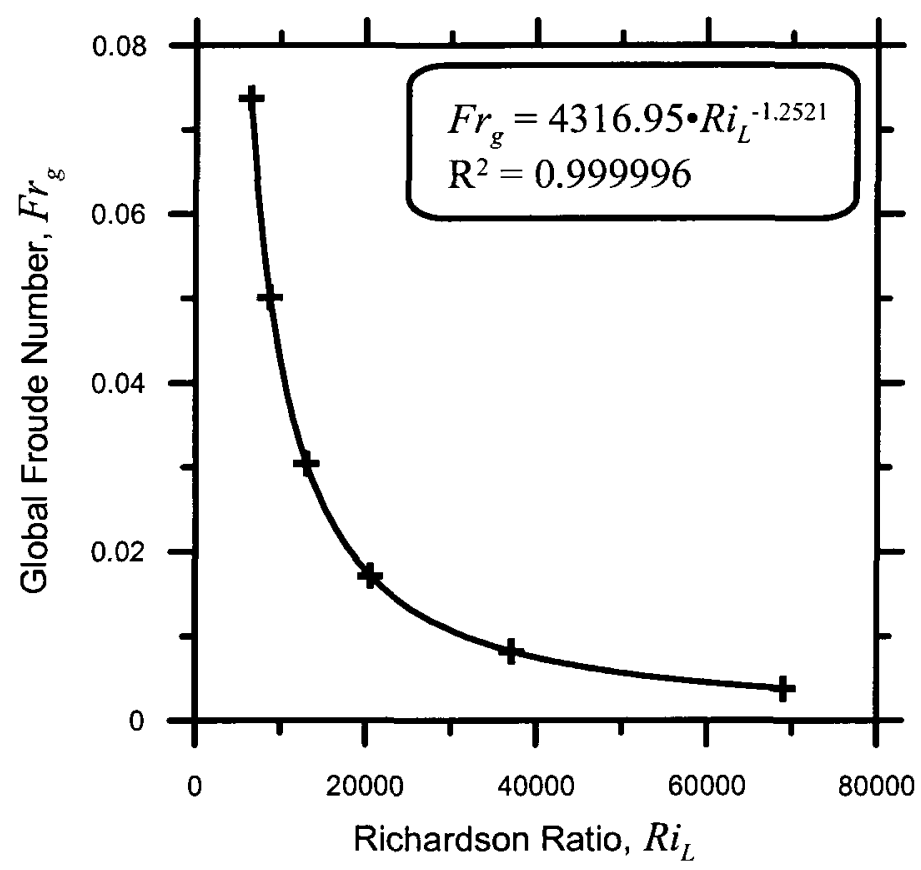

Figure 5.3 - Relationship between global Froude number and Richardson ratio as measured using methane data

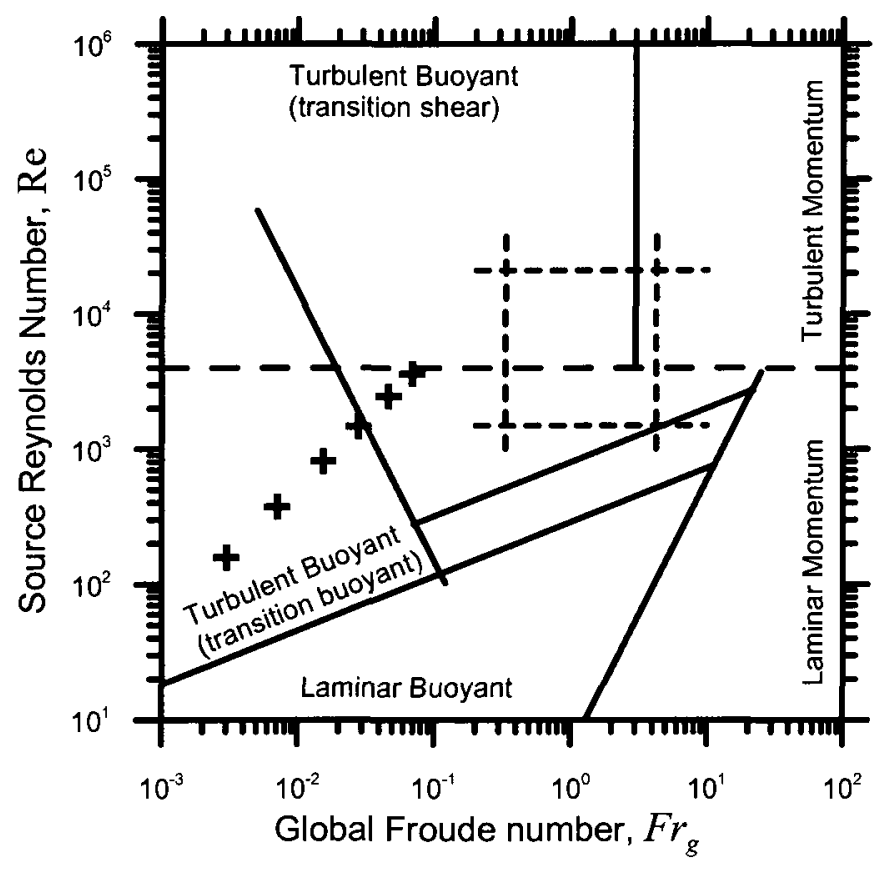

Figure 5.4 - Regime map comparing current methane data points (crosses) and the region expected to include the data of Becker and Liang [1982] (dashed lines) 


\subsection{Potential Effects of Fuel Pre-heating}

In attached (i.e. non-lifted) diffusion flames, heat transfer to the burner tip is an important component of the stabilization mechanism. While this heat transfer is an integral aspect of the flame configuration, it also leads to inherent preheating of the fuel as it travels up the burner tube. Prior to attempting to develop models to correlate current data for a range of flow conditions and burner diameters, it was important to consider potential complications from the confounding effect of variations in preheating among these same conditions.

Canteenwalla [2007] had observed from LII soot volume fraction measurements that the Lab Scale Flare system had a distinct warm-up period of approximately ten minutes, where $f_{v}$ would increase before settling to a final value. It was hypothesized that this initial increasing trend could be attributed to heating of the burner exit tube by the flame, which in turn was preheating the fuel gas stream within the burner. In his work, Canteenwalla [2007] measured heating of the burner tube for a selected test condition using K-type thermocouple probes placed 13 and $25 \mathrm{~mm}$ below the burner tip on the outside of the burner. He reported temperature increases of approximately 70 and $40 \mathrm{~K}$ above room temperature when the flame had stabilized. Canteenwalla [2007] used this information to help establish a warm-up protocol to preclude influence of start-up transients in his data.

In an attempt to gain further insight into the potential effects of fuel preheating, measurements were made of the gas temperature directly upstream of the burner exit using a K-type thermocouple probe. Details of the temperature measurement setup and calculations are provided in Appendix C. Since the temperature of the fuel and burner 
were not deliberately controlled in the current work, the gas temperature was a function of the nominal fuel volume flow rate, as shown in Figure 5.5. For all test conditions in the current data set, the largest increase in temperature above ambient was approximately $45 \mathrm{~K}$ while the minimum was $10 \mathrm{~K}$ (i.e. the measured temperature increases were within $+35 \mathrm{~K}$ for all flow conditions tested).

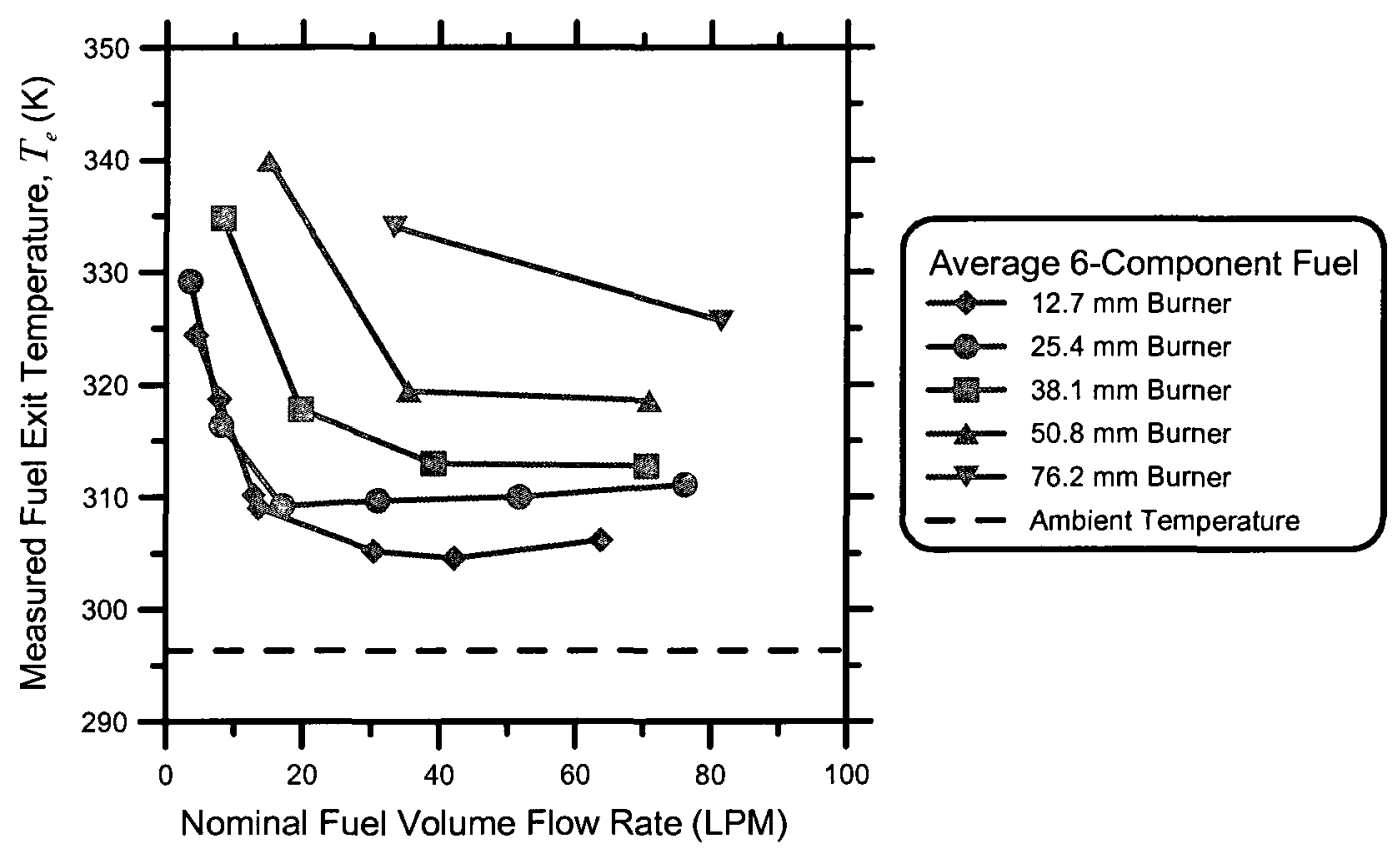

Figure 5.5 - Measured fuel temperatures $25 \mathrm{~mm}$ upstream of the burner exit for all burner diameters

The effect of fuel pre-heating has been studied in the literature for small laminar diffusion flames. Smooke et al. [1999] discovered that when they accounted for an increase in the fuel temperature, their simulations predicted the flame temperature and soot volume field inside the flame more accurately. However, their results were mainly presented with fuel and co-flow air equally heated, in contrast to the current work where preheating principally influences the fuel. Smooke et al. [1999] assumed fuel and air were preheated by $120 \mathrm{~K}$ for their analysis, $75 \mathrm{~K}$ greater than the maximum heating of $45 \mathrm{~K}$ in the current work. A sensitivity analysis showed that the pre-heated air had a much larger effect on measured soot concentration: a $164 \%$ increase in $f_{v}$ for only heated 
air, a $42 \%$ increase for only heated fuel. However, it was also noted that their model was not able to predict the peak $f_{v}$ to within a factor of three, and so their calculated preheating effect should be viewed as an indicator of trends only.

Guo et al. [2002] also studied the fuel pre-heating effect in a laminar co-flow ethylene diffusion flame, where their simulations included the heated section of the burner tip in the domain of the simulation. A linear temperature distribution was applied to the burner based on measured values from actual tests ranging from $300 \mathrm{~K}$ at $4 \mathrm{~cm}$ below the exit to $403 \mathrm{~K}$ at the burner tip. In their simulations, the conduction and convection heat transfer from the wall of the fuel nozzle and flame base to both fuel and air streams were taken into account. Simulation results showed that in these laminar co-flow flames, the average fuel temperature was predicted to increase by $131 \mathrm{~K}$, which was higher than the maximum wall temperature. This suggested that flame radiation also contributed to the fuel preheating (at least in the simulations). For the same configuration, the co-flow air average temperature was predicted to increase by $29 \mathrm{~K}$. Calculated peak soot volume fractions were $7.2 \mathrm{ppm}$ for the pre-heated case vs. $6.2 \mathrm{ppm}$ for the unheated case, suggesting an increase of $16 \%$ due to preheating of both fuel and co-flow air. However, it should be noted that both calculated values differed from the experimentally measured peak value of $8.0 \mathrm{ppm}$. Guo et al. [2002] postulated that the increase in fuel temperature caused the fuel to decompose earlier in the combustion process, producing acetylene earlier and promoting longer soot inception and growth time, thus resulting in a larger peak soot volume fraction.

Following the work of Guo et al. [2002], and conservatively assuming a linear dependence of soot formation on preheat temperature, the increase in fuel temperature of 
up to $45 \mathrm{~K}$ in the current experiments would imply an increase in the peak soot volume fraction of approximately $6 \%$ relative to a non-physical, unheated case. For temperature increases within the range of $+35 \mathrm{~K}$ noted in the current experiments, this would imply an estimated difference in $f_{v}$ of less than $4 \%$. While it is noted that the cited data were derived from numerical models of laminar ethylene diffusion flames as opposed to turbulent flames burning alkane fuels, this analysis suggests variations in fuel pre-heating among test conditions should only have a minor influence on the soot formation process. Close examination of the experimental results from the present work supports this notion, as discussed below.

Figure 5.6 displays current measured soot yield values for several burners burning the average 6-component fuel as a function of the measured fuel temperature. In the present experiments it was not possible to vary fuel preheating without also varying the nominal fuel flow rate through the burner, so the observed variations of soot yield with preheat temperature are intrinsically coupled to the fuel flow rates used to achieve the preheat. For the $12.7 \mathrm{~mm}$ burner, there is essentially no change in the measured soot yield over a range of flow rates where the measured preheat temperature varied by approximately $20 \mathrm{~K}$. For the $25.4 \mathrm{~mm}$ burner, there is a significant up-down trend, although the data points near a preheat temperature of $310 \mathrm{~K}$ cross-over themselves, indicating that temperature change must not be the primary reason for this behaviour. Together, these data suggest that the effect of preheating is either not important in the range of the current data, or is much less important than other parameters related to the flow conditions, e.g. mass flow rate or exit velocity. For the larger three burners (and a portion of the $25.4 \mathrm{~mm}$ burner data), the data suggest that the soot yield actually 
decreases with increasing fuel temperature. Since the temperature is integrally coupled with the nominal fuel flow rate, this further suggests that the flow conditions have a greater impact on the soot yield than the pre-heating.

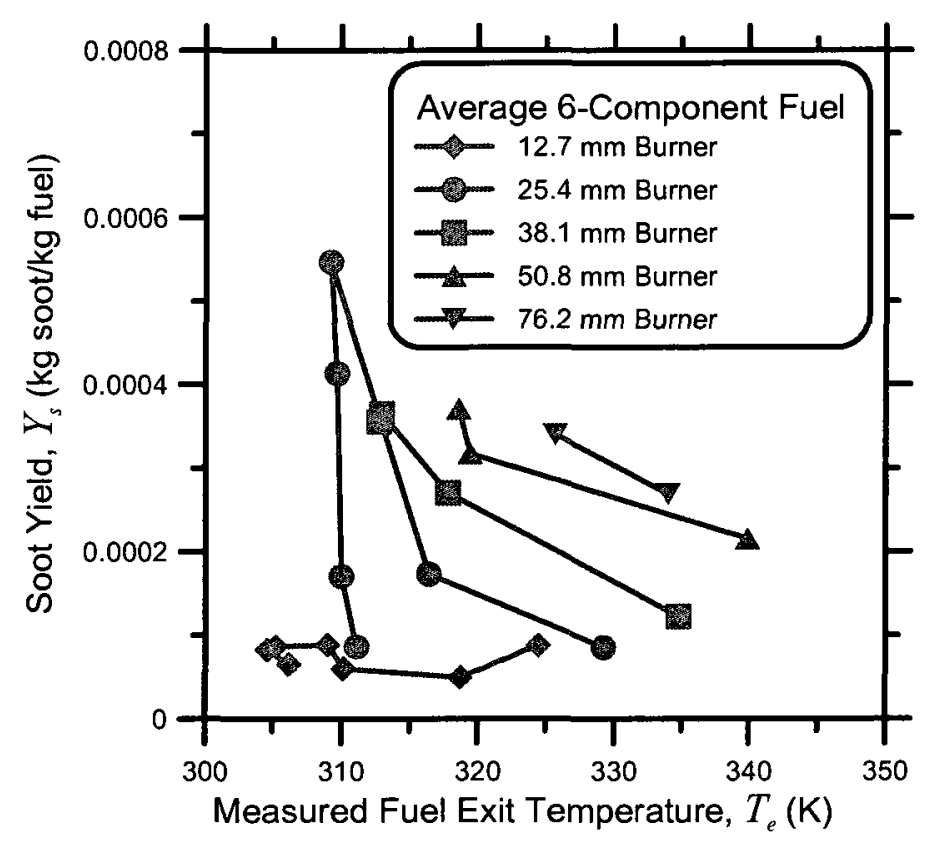

Figure 5.6 - Soot yield as a function of measured fuel temperature

The pre-heating effect will exist in real flares and most likely existed in the work of others such as Becker and Liang [1982] and Delichatsios [1993a]. Correlations they developed, including the regime map of Delichatsios [1993a], were likely based on the cold flow conditions, as there was no indication to suggest otherwise. Sivathanu and Faeth [1990] used a water-cooled burner, but there was no mention as to why, or what the temperature of the cooled burner was. Based on this and the fact that the flow conditions seem to have a greater impact than the pre-heating on soot yield, all fuel flow parameters (temperature, density, velocity) were calculated based on the ambient temperature conditions and presented as nominal values in the discussion that follows. 


\subsection{Scaling Soot Emissions}

As described in this section, an attempt was made to scale the present soot yield data based on several parameters and models found in the literature. Ideally, one or more of these models could provide a simple approach to estimating soot emission factors based on easily obtainable data such as burner size and cold flow characteristics. Unless noted otherwise, all plots are for the average 6-component fuel mixture, since this is the most complete data set. Fuel chemistry effects are discussed separately in section 5.4.

\subsubsection{Flame Regimes}

As seen in Chapter 4 and as shown in Figure 5.7, there seems to be a difference in soot yield behaviour between the $12.7 \mathrm{~mm}$ burner, the $25.4 \mathrm{~mm}$ burner, and the three larger burners. As discussed in Chapter 2, the work of Delichatsios [1993a] identified several regimes of turbulent diffusion flames. Figure 5.8 shows data points for the full range of current test conditions in which the average 6-component mixture was used as a fuel. The hatched rectangle shows the estimated regime of solution gas flares typical of Alberta, as presented previously in Chapter 2. While only a few of the current data points fall directly in the area expected to be representative of solution gas flares, all data are in the turbulent-buoyant regime and both data sets span the transition-buoyant and transition-shear sub-regimes. 


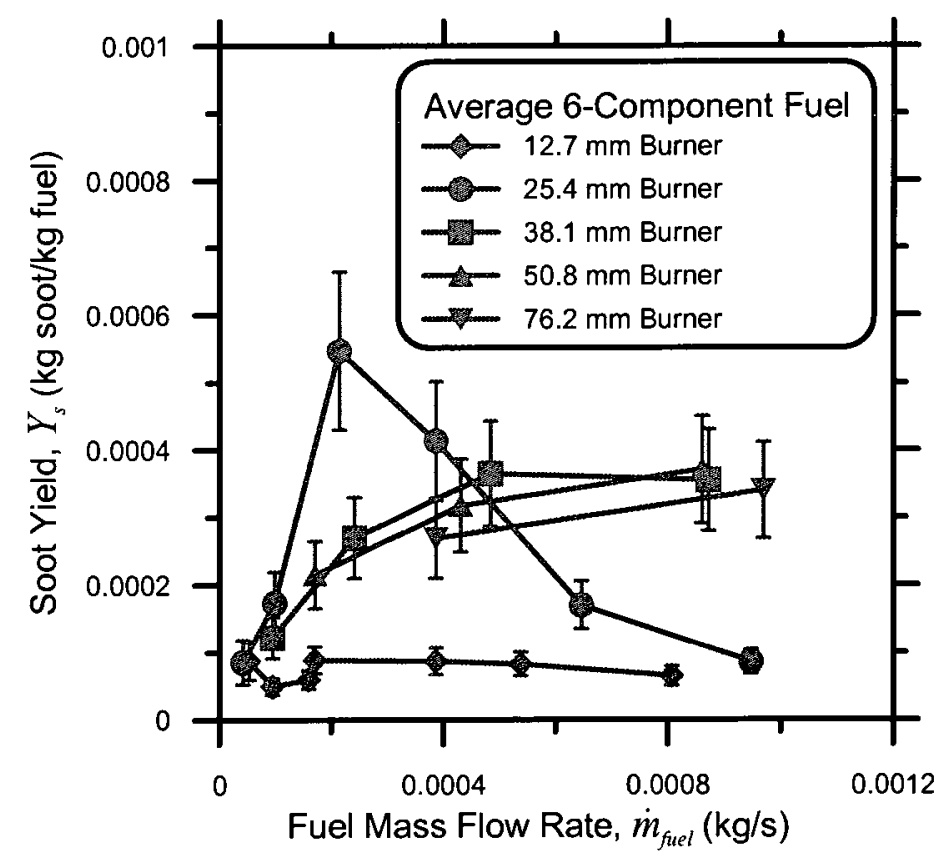

Figure 5.7 - Soot yield vs. mass flow rate for all diameters burning the AVG-6-component fuel mixture

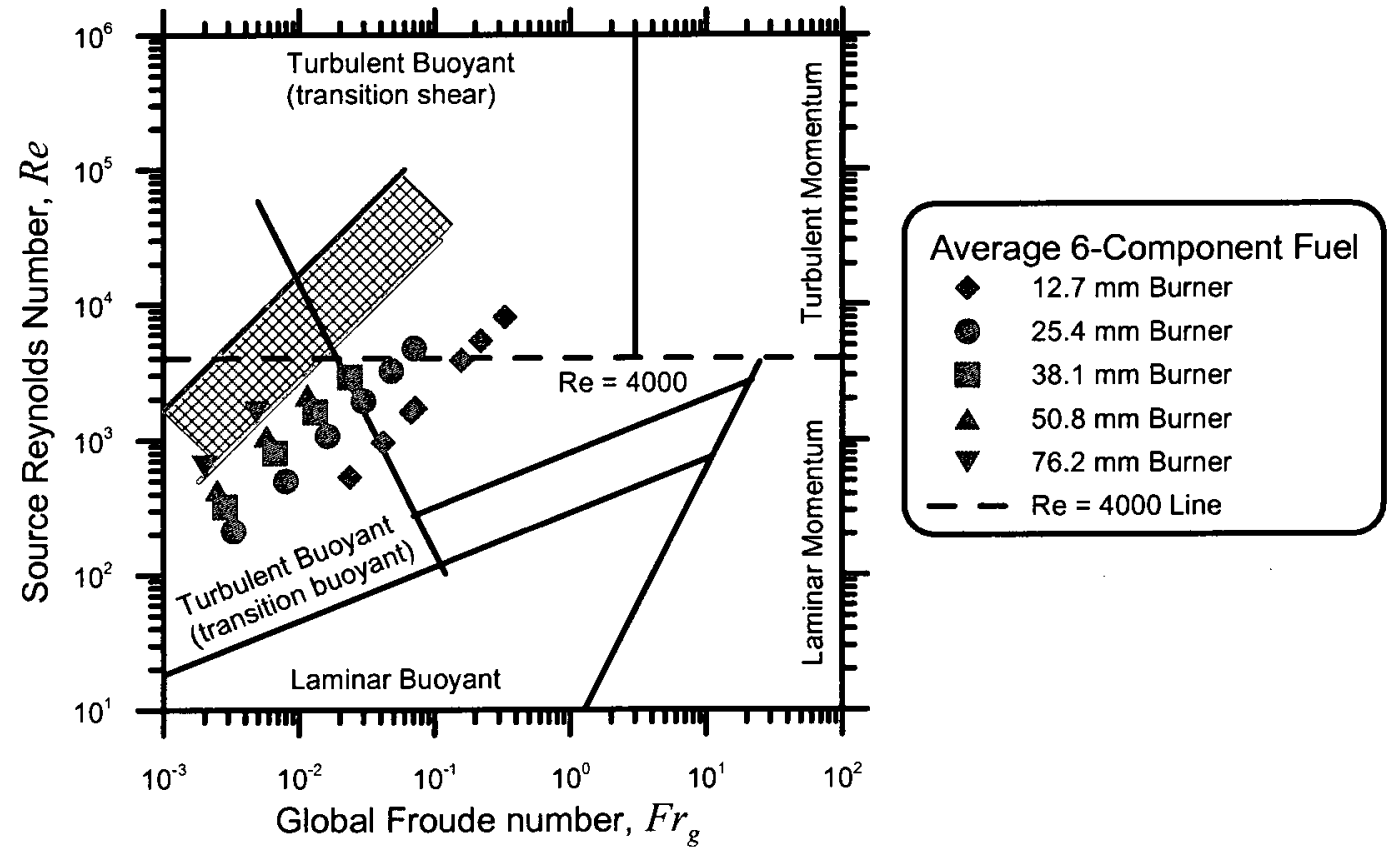

Figure 5.8 - Regime map showing expected flare operation between red and blue lines, and current data for the AVG-6-component fuel mixture

Figure 5.9 displays a subset of the soot yield data corresponding to the transitionbuoyant sub-regime extracted from all available data for the average 6-component fuel mixture (all burner diameters and exit velocities where appropriate). For data in this sub- 
regime, there are no decreasing trends on the graph; values appear to be increasing to some unknown maximum value for the $25.4 \mathrm{~mm}$ burner, and approaching some asymptotic value for the three largest burners. For fuel mass flow. rates where the data for different burners overlap, soot yield values were consistent among the results for the different burner diameters. This is especially true for the three largest burner diameters. Conversely, the soot yield for the $25.4 \mathrm{~mm}$ diameter burner at a fuel mass flow rate of $0.0002 \mathrm{~kg} / \mathrm{s}$ deviates from the other data.

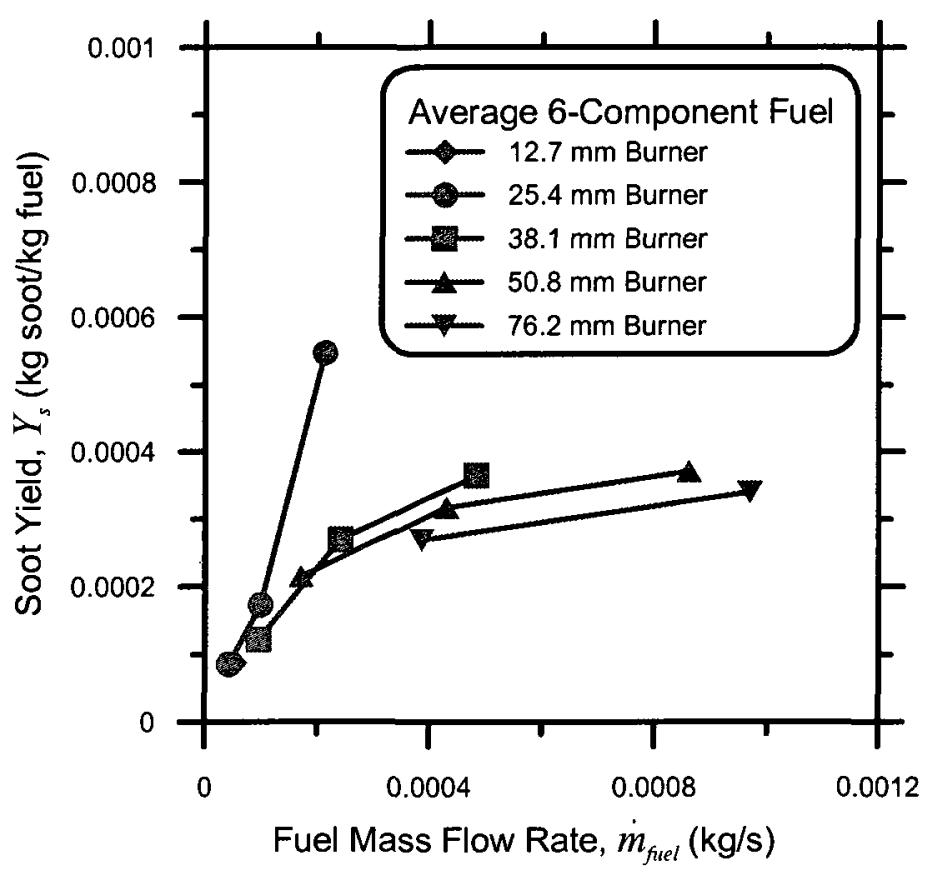

Figure 5.9 - Soot yield for all burners burning the AVG-6-component fuel mixture for the transitionbuoyant sub-regime

Figure 5.10 displays all soot yield data for the transition-shear sub-regime of the average 6-component fuel mixture. In contrast to the transition-buoyant sub-regime, the $25.4 \mathrm{~mm}$ diameter burner data show a decreasing soot yield with increasing fuel mass flow rate. The different behaviour of the transition-shear sub-regime may be related to a difference in the amount of turbulent mixing. The crosswind results of Ellzey et al. [1990] and Prybysh [2002] showed that the increased mixing decreased the soot yield, 
and perhaps the same trend exists here if there is indeed increased mixing. The data for the $12.7 \mathrm{~mm}$ diameter burner show minimal change through the full range of mass flow rates.

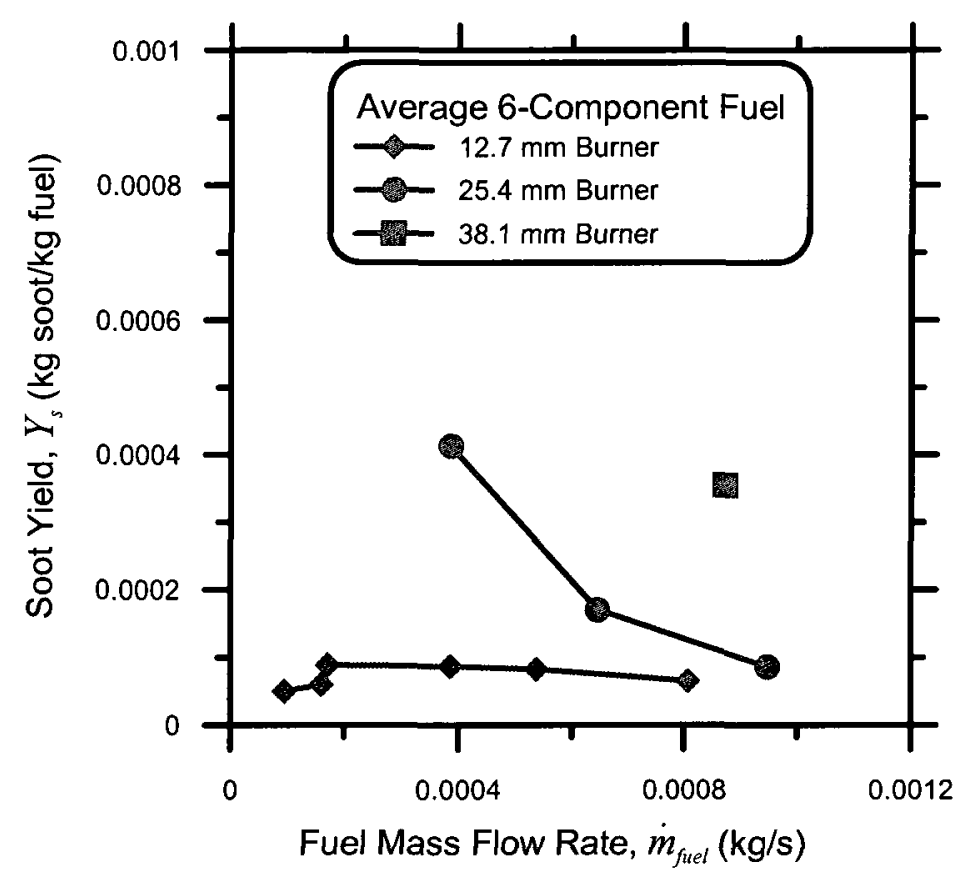

Figure 5.10 - Soot yield for all burners burning the AVG-6-component fuel mixture for the transition-shear sub-regime

While there are only two data points for the $76.2 \mathrm{~mm}$ burner in Figure 5.9, the data are closely aligned with the 50.8 and $38.1 \mathrm{~mm}$ burners. The $12.7 \mathrm{~mm}$ data fall in the transition-shear sub-regime as observed in Figure 5.10, which may explain why the soot yield does not follow the similar increasing trends of the other burner diameters, while the $25.4 \mathrm{~mm}$ data show opposing trends in the different sub-regimes. There is an obvious difference in soot yield behaviour at different flow conditions, but since the transition line defined by Delichatsios [1993a] is not necessarily a well-defined transition (being based on visual observations), the data will be shown separated by diameter. Results will be presented henceforth with dual plots: one containing all data and one containing only the three largest diameter burners. It is expected that the three largest burners will be more 
representative of full-scale flares, while the smaller diameter data may provide further insight in the potential importance of regime differences.

\subsubsection{Aerodynamic Scaling Parameters}

The fire Froude number suggested by Delichatsios [1993a] was used in an attempt to scale soot yield values. Figure 5.11 (a) displays the soot yield data for all burners and illustrates that there is no single trend among the data as a function of fire Froude number. However Figure 5.11(b), which plots only data from the three larger lab-scale flares $(38.1,50.8,76.2 \mathrm{~mm})$, illustrates a common trend within this limited data set: the soot yield appears to be increasing to a horizontally asymptotic value of $\sim 0.0004 \mathrm{~kg}$ soot $/ \mathrm{kg}$ fuel at a fire Froude number of $\sim 0.005$. Although the available data are quite limited, these results suggest there may be a transition in soot yield behaviour over the range of $0.003 \leq F r_{f} \leq 0.005$. If true, this implies that a typical $101.6 \mathrm{~mm}$ flare operating at flow rates up to approximately $400 \mathrm{LPM}^{1}$ would have an increasing soot yield with increasing flow rate, and that above 400 LPM, the soot yield would remain constant.

The Richardson ratio defined by Becker and Liang [1982] was also tested as a scaling parameter. As illustrated in Figure 5.12, there is again a difference in behaviour with the 12.7 and $25.4 \mathrm{~mm}$ burners, compared to the three larger burners. The three larger burners (Figure 5.12 (b)) show a consistent trend of linearly decreasing soot yield with increasing Richardson ratio. When viewed on a log scale (Figure 5.12 (a)), the three largest burners appear to be approaching the same asymptotic value mentioned previously $(\sim 0.0004 \mathrm{~kg}$ soot $/ \mathrm{kg}$ fuel) as the Richardson ratio decreases to a value of

\footnotetext{
${ }^{1}$ Calculated based on the AVG-6-component fuel mixture properties and a burner exit velocity of $0.8 \mathrm{~m} / \mathrm{s}$.
} 
approximately 20,000. A second parameter suggested by Becker and Liang [1982] for scaling soot emissions was a global characteristic residence time, $\tau_{R}^{*}$. Figure 5.13 shows soot yield as a function of global characteristic residence time, but no useful trends are discernable.

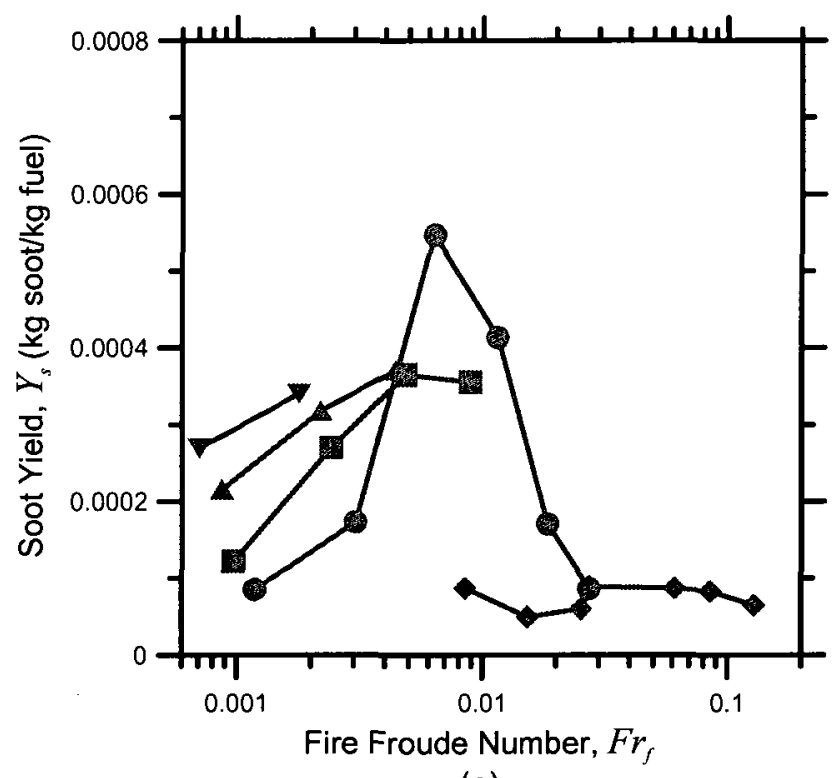

(a)

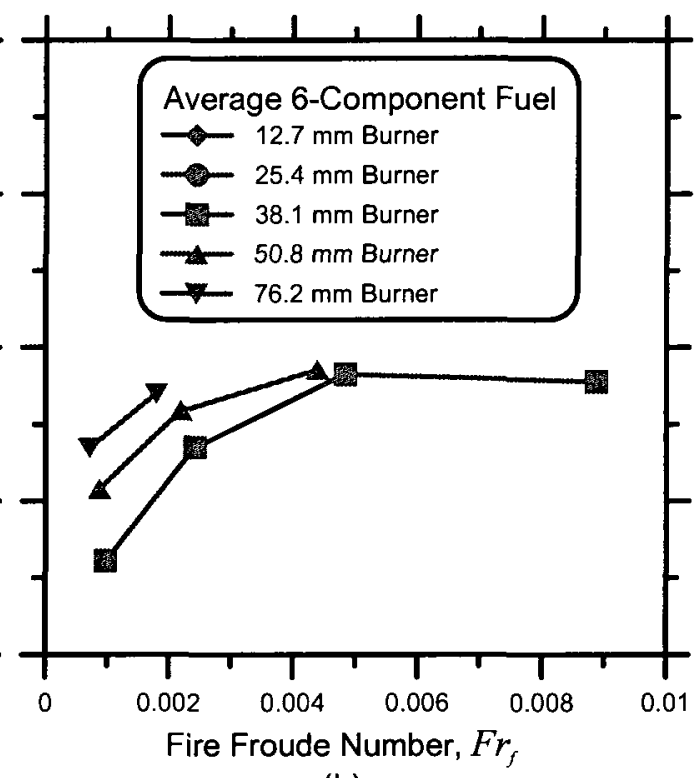

(b)

Figure 5.11 - Soot yield as a function of the fire Froude number for (a) all burners and (b) the three largest burners burning the AVG-6-component fuel. $F r_{f}=u_{e} f_{s}^{3 / 2}\left(\rho_{e} / \rho_{\infty}\right)^{-1 / 4}\left(g d_{e} \Delta T_{f} / T_{\infty}\right)^{-1 / 2}$ 


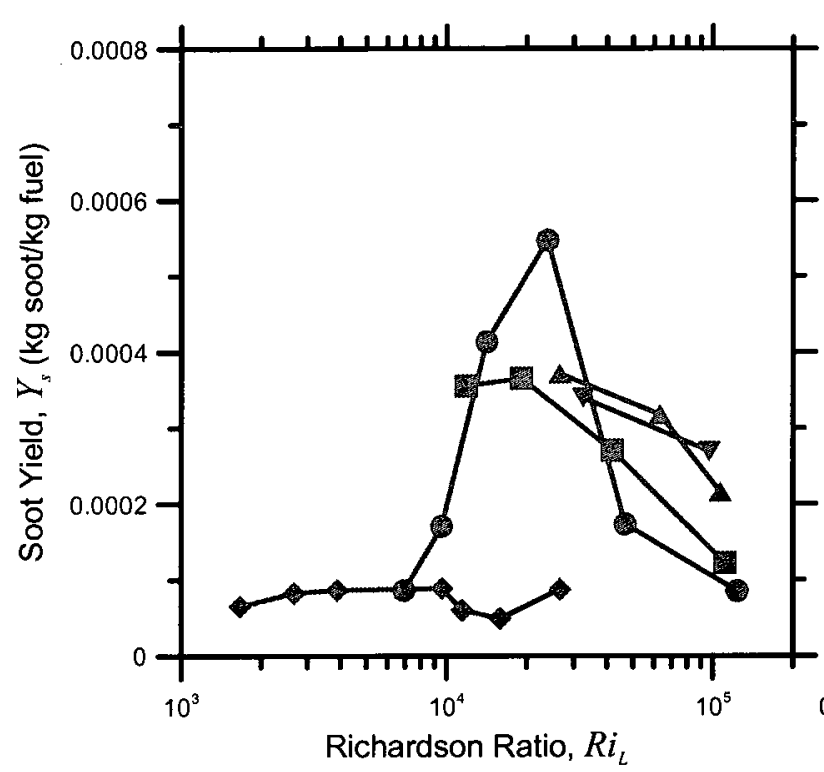

(a)

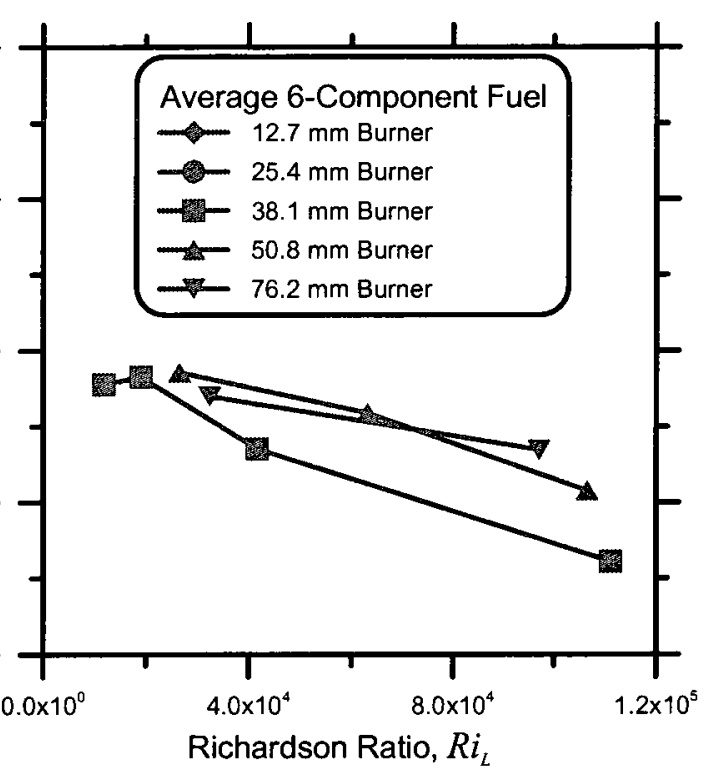

(b)

Figure 5.12 - Soot yield as a function of the Richardson ratio as defined by Becker and Liang [1982] for (a) all burner diameters and (b) the three largest burner diameters burning the AVG-6component fuel. $R i_{L}=g L_{f}^{3}\left(u_{e} d_{e}\right)^{-2} \rho_{\infty} / \rho_{e}$

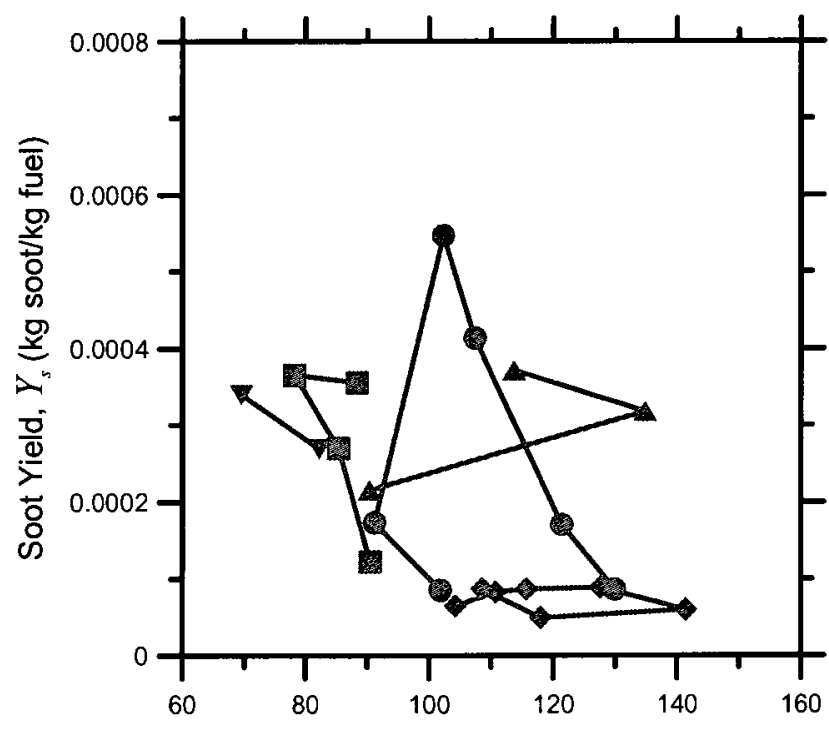

Global Characteristic Residence Time, $\tau_{R}^{*}(\mathrm{~s})$

(a)

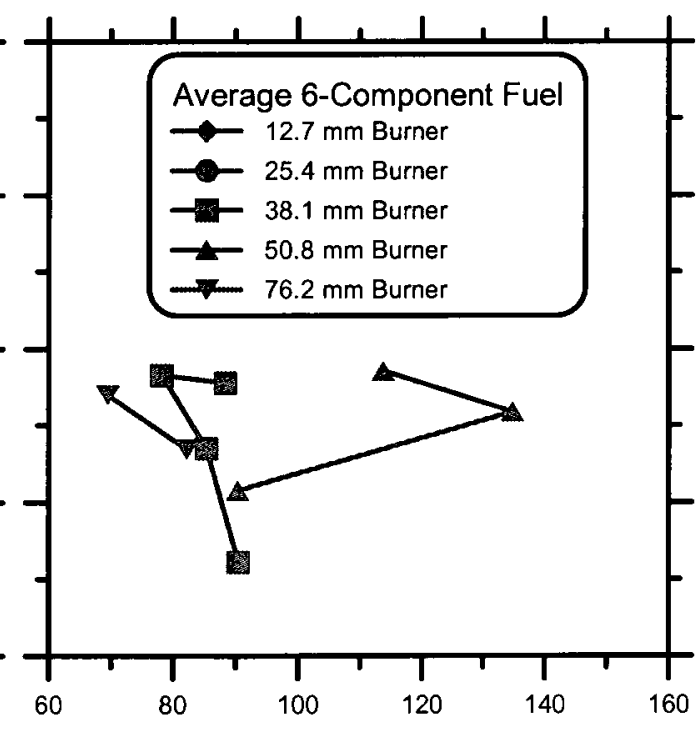

Global Characteristic Residence Time, $\tau_{R}^{*}(s)$

(b)

Figure 5.13 - Soot yield as a function of the global characteristic residence time $\left(\tau_{R}^{*}=\rho_{\infty} f_{s} L_{f}^{3} / \dot{m}_{\text {fitel }}\right)$ as defined by Becker and Liang [1982] for (a) all burner diameters and (b) the three largest burner diameters burning the AVG-6-component fuel.

Canteenwalla [2007] suggested a buoyant residence time for scaling soot emissions.

This residence time calculation (Equation 2.14) was based on constant buoyancy driven 
acceleration (Equation 2.15). The present data were plotted as a function of the buoyancy residence time in Figure 5.14. Curiously, the data for the $50.8 \mathrm{~mm}$ and $76.2 \mathrm{~mm}$ burners appear to lie on a straight line. This could be attributed to the fact that these burners have not reached the plateau region that the $38.1 \mathrm{~mm}$ burner exhibits, or could be coincidental.

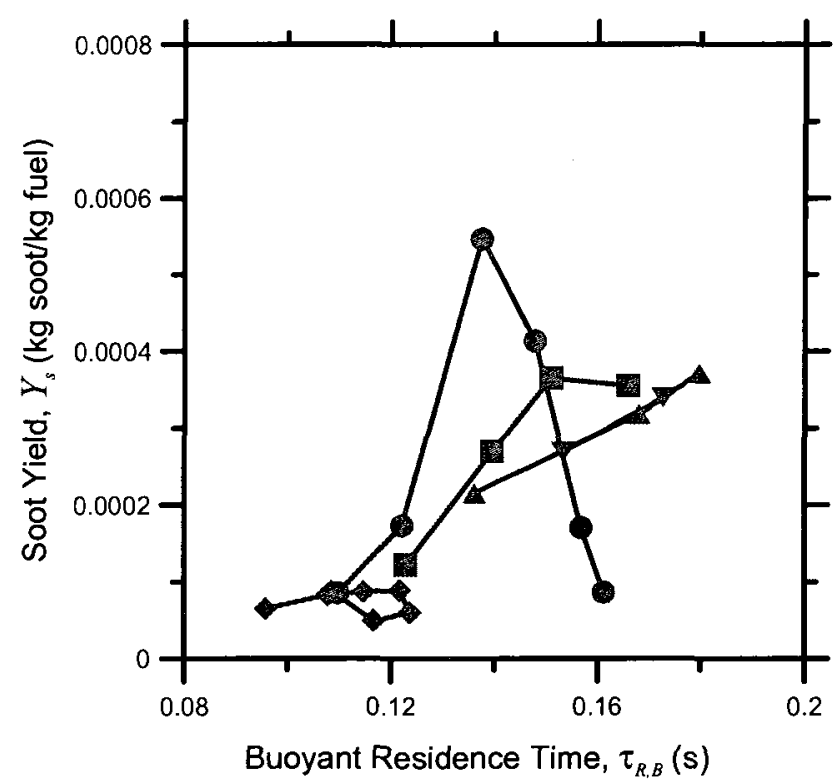

(a)

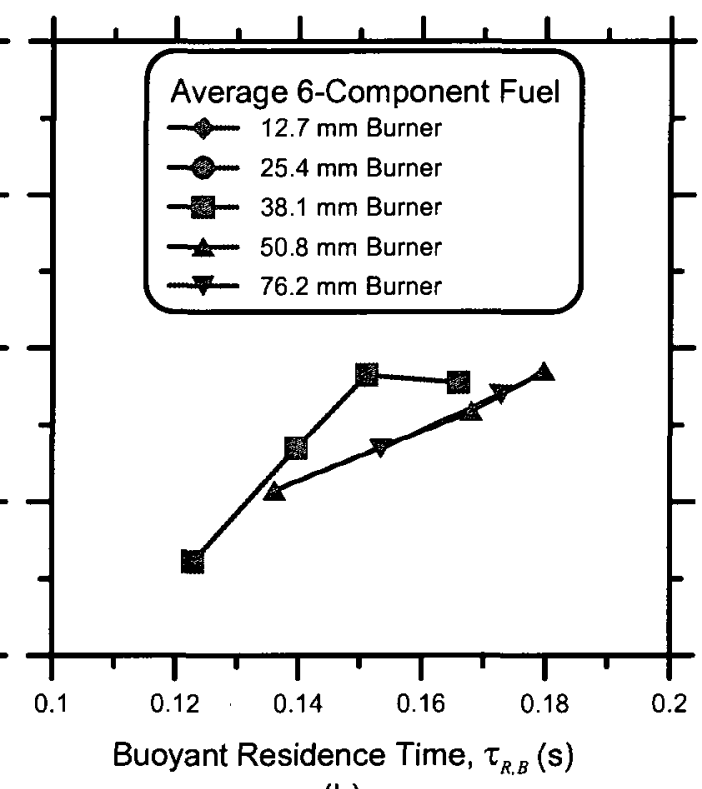

(b)

Figure 5.14 - Soot yield as a function of the buoyant residence time defined by Canteenwalla [2007] for (a) all burner diameters and (b) the largest three burner diameters burning the

$$
\text { AVG-6-component fuel. } \tau_{R, B}=\left(-u_{e}+\sqrt{u_{e}^{2}+2 a_{B} L_{f}}\right) / a_{B}
$$

Based on the preceding analysis, the fire Froude number is a reasonable scaling parameter to consider when attempting to understand variations in soot yield at different flow conditions. From the figures presented previously, it appears that for the largest three burners, the soot yield approaches an asymptotic value as the fire Froude number increases beyond approximately 0.003 . The conclusion that the three largest burners behave differently from the two smallest burners, while evident, must be viewed cautiously because of the limited available data. This is especially true for the $76.2 \mathrm{~mm}$ diameter burner for which there are only two data points available. 
While all of the tested flames would be classed as turbulent-buoyant, from these limited data it appears that the differences among the smallest two and largest three burners are indicative of a regime difference, and although not exact, this segregation approximately corresponds to the transition between transition-buoyant and transitionshear sub-regimes noted by Delichatsios [1993a].

From a practical perspective, the fire Froude number can be calculated using readily obtainable data such as flow rate and burner size. Although other scaling parameters (e.g. Richardson ratio and buoyant residence time) showed similar trends to that of the fire Froude number, they typically require additional information (e.g. flame length) to be measured.

\subsection{Fuel Chemistry Effects}

The influence of fuel chemistry on the chemical mechanisms of soot formation is exceedingly complex and to date not fully understood. However, in line with the practically-motivated objective of developing useable emission models for flares, it is important to seek a semi-empirical or empirical approach to modelling the effects of fuel composition on soot yield. In engineering practice, experimentally-derived smoke-point or sooting propensity data are often used to assess behaviour of different fuels. In this section, correlations of the soot yield with fuel parameters calculated from basic fuel chemistry information and combustion knowledge are explored.

Thus far, the majority of soot emission results have been presented in terms of the soot yield, which has units of mass of soot produced per mass of fuel combusted. As mentioned previously in this chapter, Becker and Liang [1982] presented their soot emission results in terms of the soot generation efficiency, $S G E$. It was thought that this 
emission factor should better represent the conversion of fuel carbon to soot carbon. The two emission factors are compared below in Figure 5.15 for the three large burners (38.1 mm, $50.8 \mathrm{~mm}$, and $76.2 \mathrm{~mm}$ ) burning the AVG- 6- and 4-component mixtures, as well as the L- and H- 4-component mixtures. In this plot the $S G E$ increases the separation between the different fuel curves rather than collapsing them as was originally hoped.

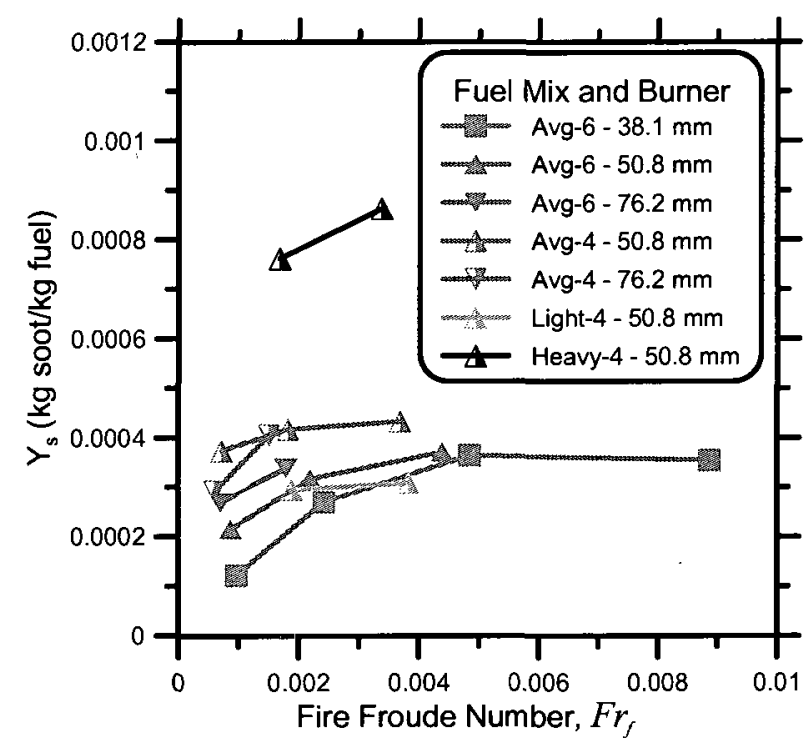

(a)

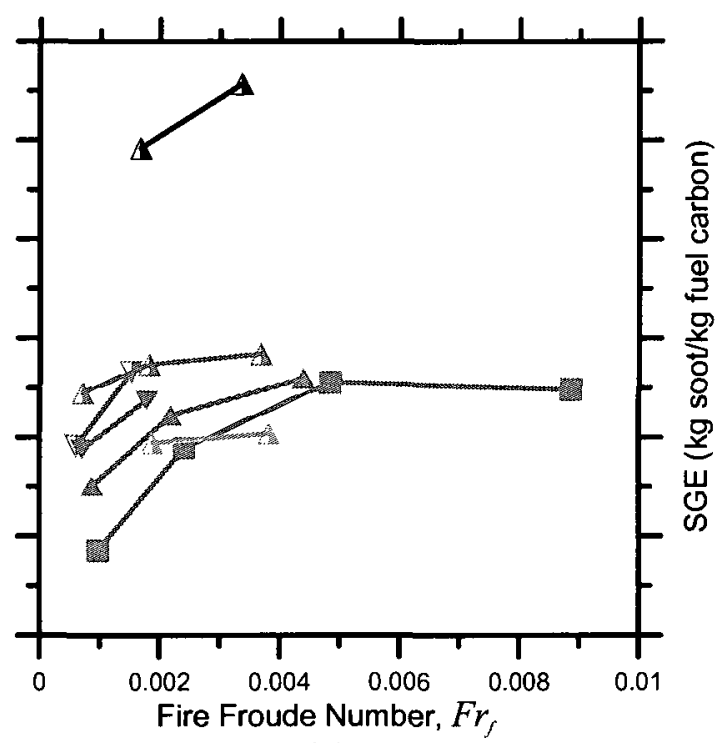

(b)

Figure 5.15 - (a) soot yield and (b) soot generation efficiency as a function of the fire Froude number for the $38.1 \mathrm{~mm}, 50.8 \mathrm{~mm}$, and $76.2 \mathrm{~mm}$ burners burning the fuel mixtures as identified in the legend. The ordinate scales are identical for both plots

In Figure 5.16, a smoke-point corrected version of the soot yield and soot generation efficiency is shown, as suggested by Canteenwalla [2007]. In his work, Canteenwalla noted that the smoke-point corrected soot yield effectively correlated results for fuel mixtures with components that did not exhibit a synergistic soot yield effect. However, he concluded that this parameter did not correlate the soot yield for mixtures containing methane and ethane. Considering that all the current mixtures contain methane and ethane, it is not surprising that the smoke-point corrected emission 
factors do not improve fuel scaling. Since neither of these alternative formulations of the soot yield improves the correlation for different fuels, the simple soot yield parameter $\left(Y_{s}\right.$, from Equation 2.1) was used in the discussion that follows.

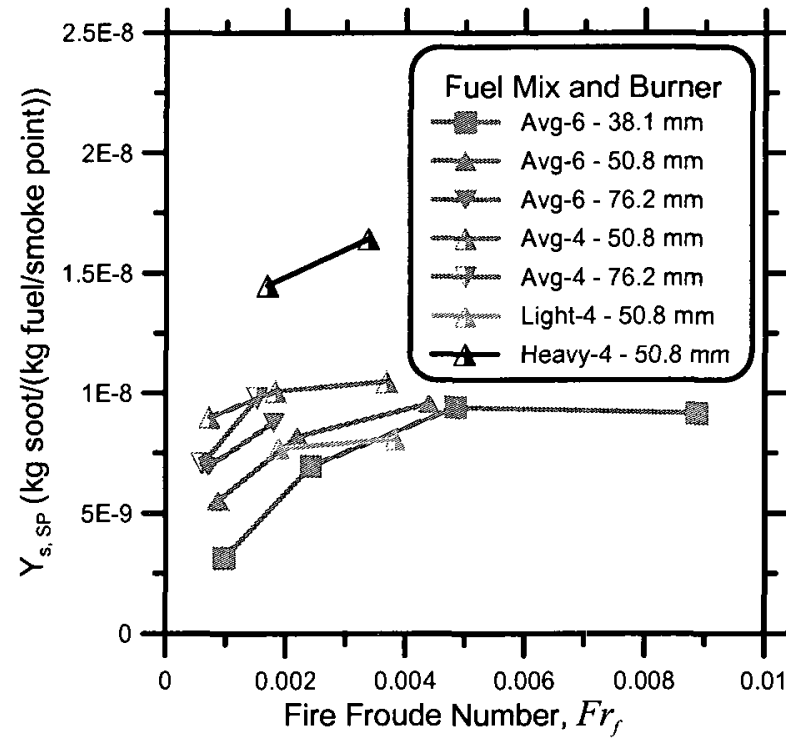

(a)

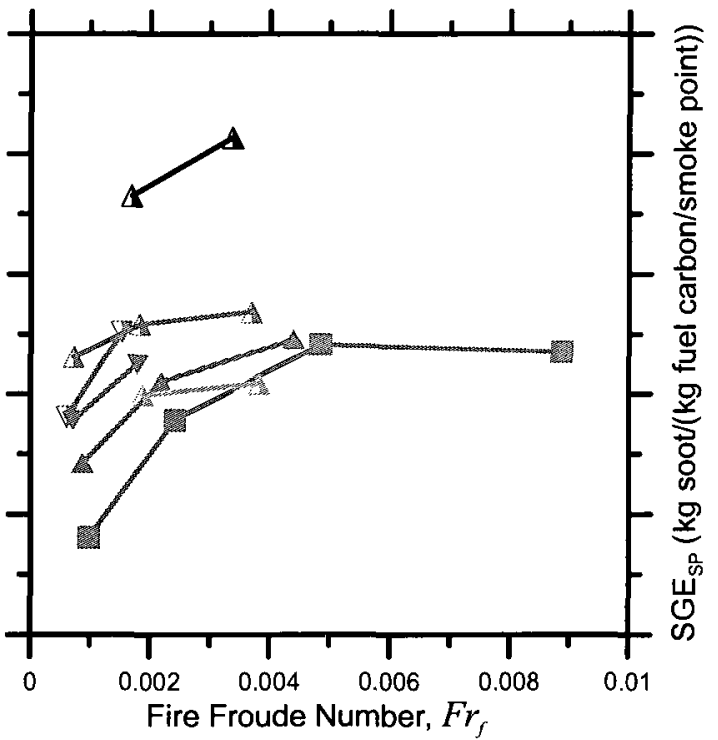

(b)

Figure 5.16 - Smoke-point corrected (a) soot yield and (b) soot generation efficiency as a function of the fire Froude number for the $38.1 \mathrm{~mm}, 50.8 \mathrm{~mm}$, and $76.2 \mathrm{~mm}$ burners burning the fuel mixtures as identified in the legend. The ordinate scales are identical for both plots

Figure 5.11 (b) demonstrated that soot yield appears to approach a constant value for $F r_{f}$ values greater than $\sim 0.005$, for the three largest burners tested. With the caveat that the data set is quite small, Figure 5.15 (a) shows a similar trend for the different fuel mixtures and suggests that the transition to a constant soot yield would occur at a $F r_{f}$ value of approximately 0.003 for the data shown. Up to a $F r_{f}$ number of 0.003 , the soot yield increases at different rates with increasing $F r_{f}$ for each burner diameter. Again noting that the observations are based on a limited data set, this again suggests that there might be two behaviours separated by some transition region spanning $F r_{f}$ values of approximately $0.003-0.005$. 
The nominal fire Froude numbers for the transition from the transition-buoyant to the transition-shear sub-regime vary slightly with Reynolds number and can be found from the regime map suggested by Delichatsios [1993a]. As shown in Figure 5.17, the transition does not occur at a single global Froude number, but rather ranges from approximately $0.013-0.025$ depending on the burner diameter. These global Froude number values correspond to fire Froude number values of $0.0045-0.0088$.

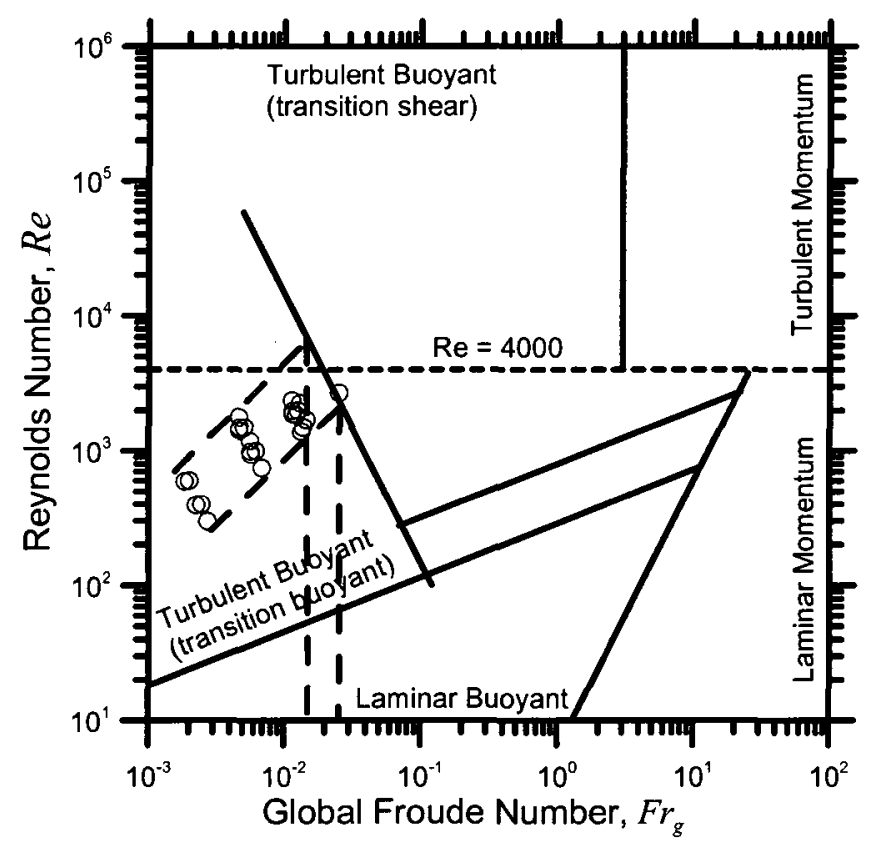

Figure 5.17 - Regime map of all data for the three largest burners burning all fuel mixtures

This nominal transition region is displayed in Figure 5.18 as the shaded area. It is evident from Figure 5.18 that the "plateau" region of soot yield for the $38.1 \mathrm{~mm}$ burner diameter burning the AVG-6-component fuel mixture is contained in the nominal transition region. As noted by Delichatsios [1993a], the transition from transition-buoyant to transition-shear turbulence is likely to be smooth and should not be limited to a single line, suggesting that the transition region could have an even wider 
range of $F r_{f}$ values, and could include numbers down to $0.003^{1}$. Notably, this transition region is close to the nominal Reynolds number transition value for turbulent pipe flow as shown in Figure 5.17; however, the effects of the cold flow turbulence should not be significant since turbulence-generating plates were used in the burner tubes and the cold flow fuel can be considered fully turbulent even at low Reynolds numbers. Cold flow turbulence intensities at the burner exit measured between 1 and $6 \%$ for the flow conditions listed in Appendix A of Canteenwalla [2007], with typical values of approximately $4-5 \%$.

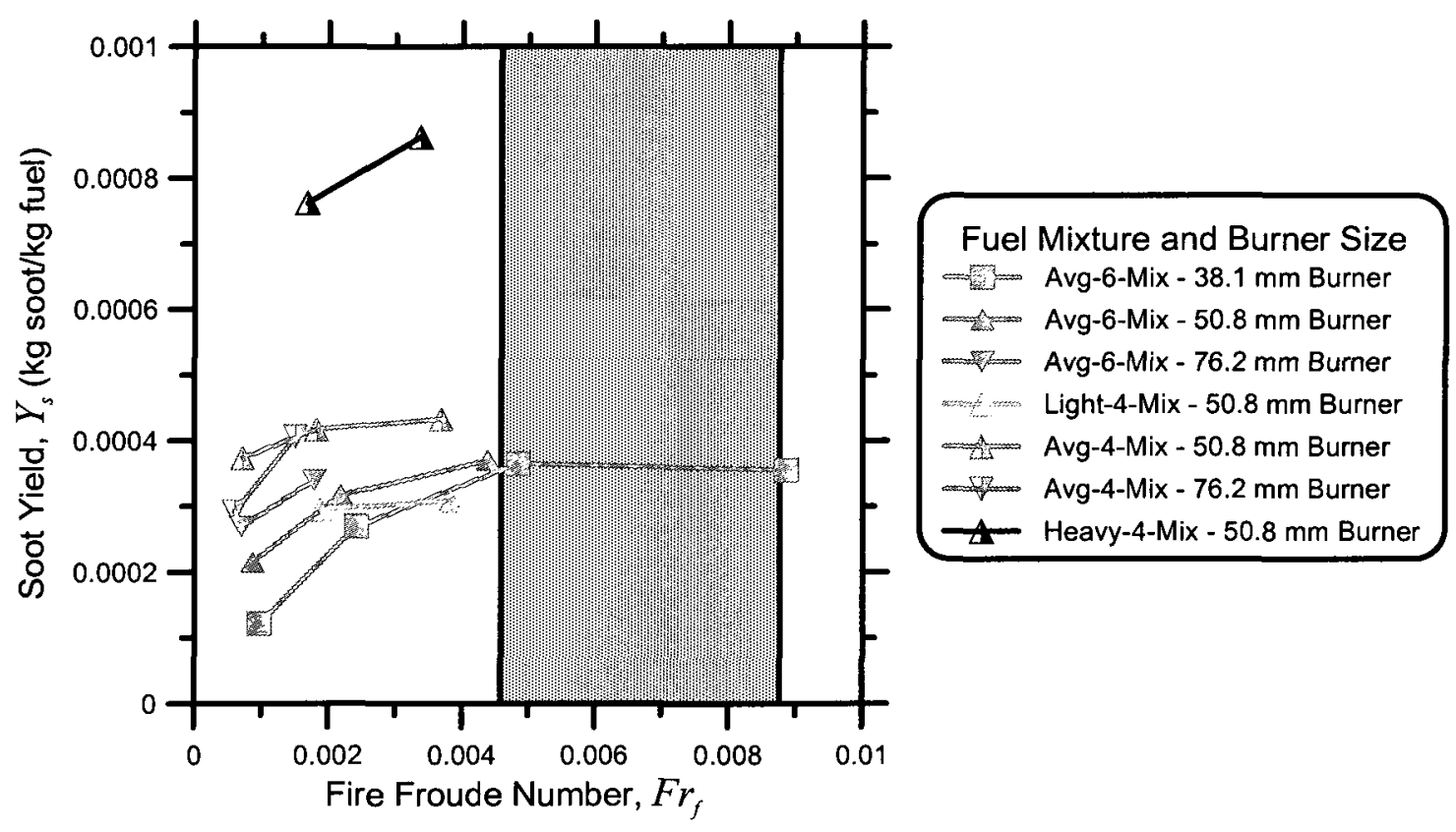

Figure 5.18 - Soot yield as a function of the fire Froude number for the three largest burner diameters and all fuel mixtures. Hatched area is the transition region as determined from Figure 5.17

\subsubsection{Chemistry-Related Scaling Parameters}

Several approaches to scaling data for different fuel mixtures at fixed flow conditions as suggested in the literature were examined. Delichatsios [1993b] suggested a parameter,

\footnotetext{
${ }^{1}$ This fire Froude number corresponds to a global Froude number of approximately 0.009 .
} 
which will be referred to as the Delichatsios parameter, $D_{p}$, based on: the heat of combustion (or heating value); a smoke-point mass flow rate, $\dot{S}_{s p}$; and the stoichiometric mixture fraction, $f_{s}$ (Equation 5.1). Figure 5.19 shows current data for constant flow conditions (i.e. fixed exit velocity and burner diameter) plotted separately for conditions with $F r_{f}<0.003$ (Figure 5.19 (a)) and $F r_{f}>0.003$ (Figure 5.19 (b)). Data points at conditions where only one fuel was used were not plotted (three points in total), so that the effectiveness of the parameter in modelling fuel effects could be more clearly seen.

$$
D_{p}=\dot{S}_{s p} f_{s}
$$

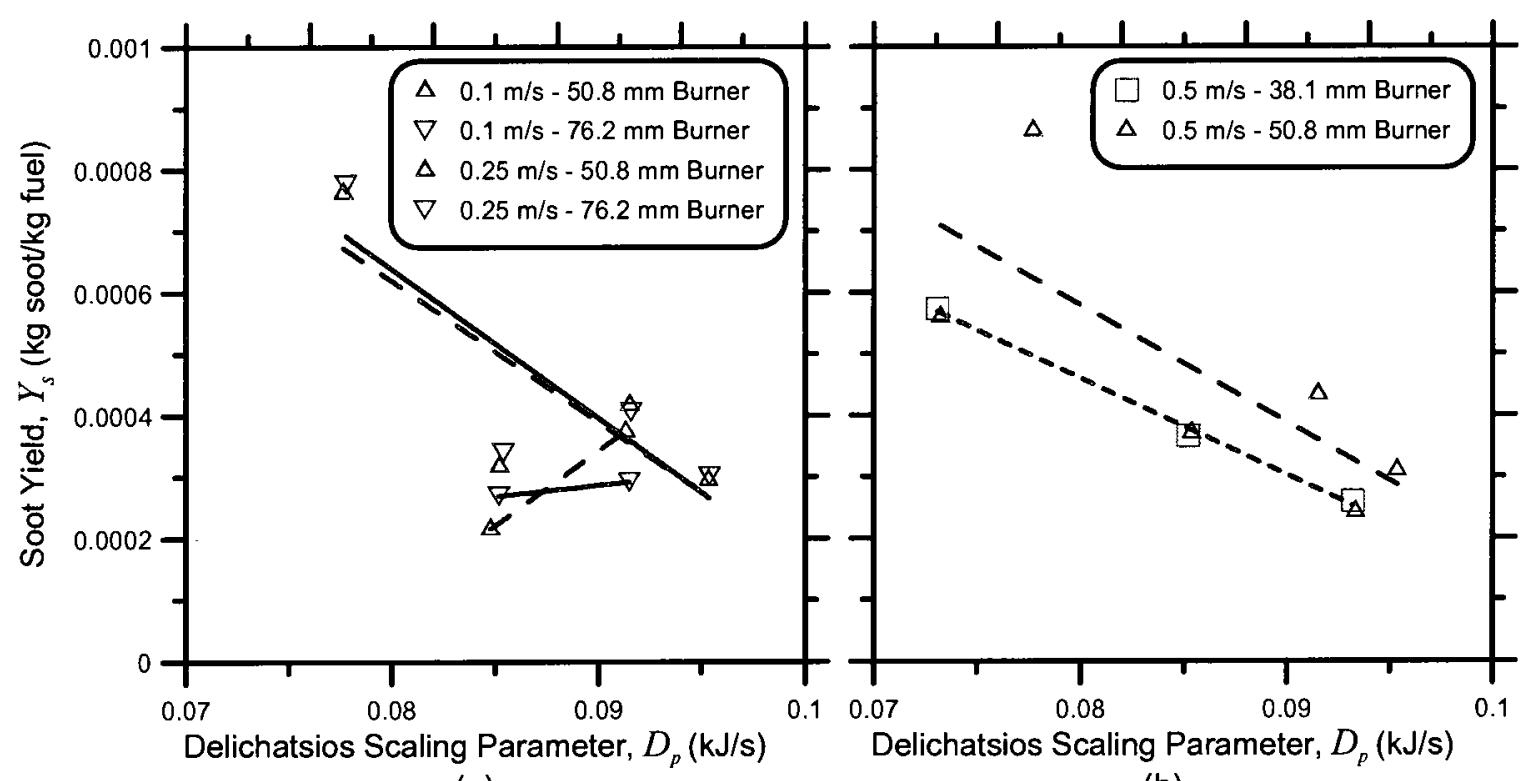

(a)

(b)

Figure 5.19 - Soot yield as a function of the Delichatsios scaling parameter for values with a $F r_{f}$ of (a) less than 0.003 and (b) greater than 0.003 . Lines represent linear fits of data.

From Figure 5.19 (b), the scaling parameter suggested by Delichatsios [1993b] reasonably fits the fuel data for constant flow conditions. It fails, however, to collapse data for different flow conditions as observed in Figure 5.19 (a), where the different exit velocities have both positive and negative slopes for exit velocities of $0.1 \mathrm{~m} / \mathrm{s}$ and $0.25 \mathrm{~m} / \mathrm{s}$, respectively. While the slopes of these curves in Figure 5.19 (a) were expected 
to differ at $F r_{f}<0.003$ consistent with the previous discussion, it was expected that the slopes would either be both positive or both negative. It was therefore concluded that the Delichatsios scaling parameter may not be a reasonable scaling factor for the current data. A plot of the soot yield as a function of only the smoke-point was also considered, but the trends of this figure were identical to those of Figure 5.19. As mentioned in Chapter 2, the difficulty in calculating a smoke-point mass flow rate limits the effectiveness of this scaling parameter.

Glassman [1998] suggested that the flame temperature and the residence time of soot within the elevated temperature region has an important effect on the soot formation. In light of this suggestion, soot yield was plotted as a function of the adiabatic flame temperature, as shown in Figure 5.20. Adiabatic flame temperatures were calculated for all fuel mixtures using the chemical equilibrium software CEA (NASA Glenn Research Center [2004]). Figure 5.20 (a) displays all data points for the data with $F r_{f}<0.003$ (including the three points omitted in Figure 5.19), while Figure 5.20 (b) displays all data points for the data with $F r_{f}>0.003$. It appears that the adiabatic flame temperature does a better job than $D_{p}$ in scaling soot yields for different fuels, as the slopes of linear fits in the graphs are similar and are all positive. However, the slopes of the linear fits for Figure $5.20(\mathrm{~b})$ are not consistent, which suggests that the adiabatic flame temperature alone may not be able to describe the sooting behaviour with different burner diameters.

Referring back to Chapter 1, where current soot emission factors were listed, the emission factor suggested by the CAPP guide [2007] was reported as a corrected version of a USEPA factor. The correction was applied based on the volumetric heating value to 
a standard UOG solution gas heating value of $45,000 \mathrm{~kJ} / \mathrm{m}^{3}$. Using the same principle, soot yield versus volumetric heating value is shown in Figure 5.21.

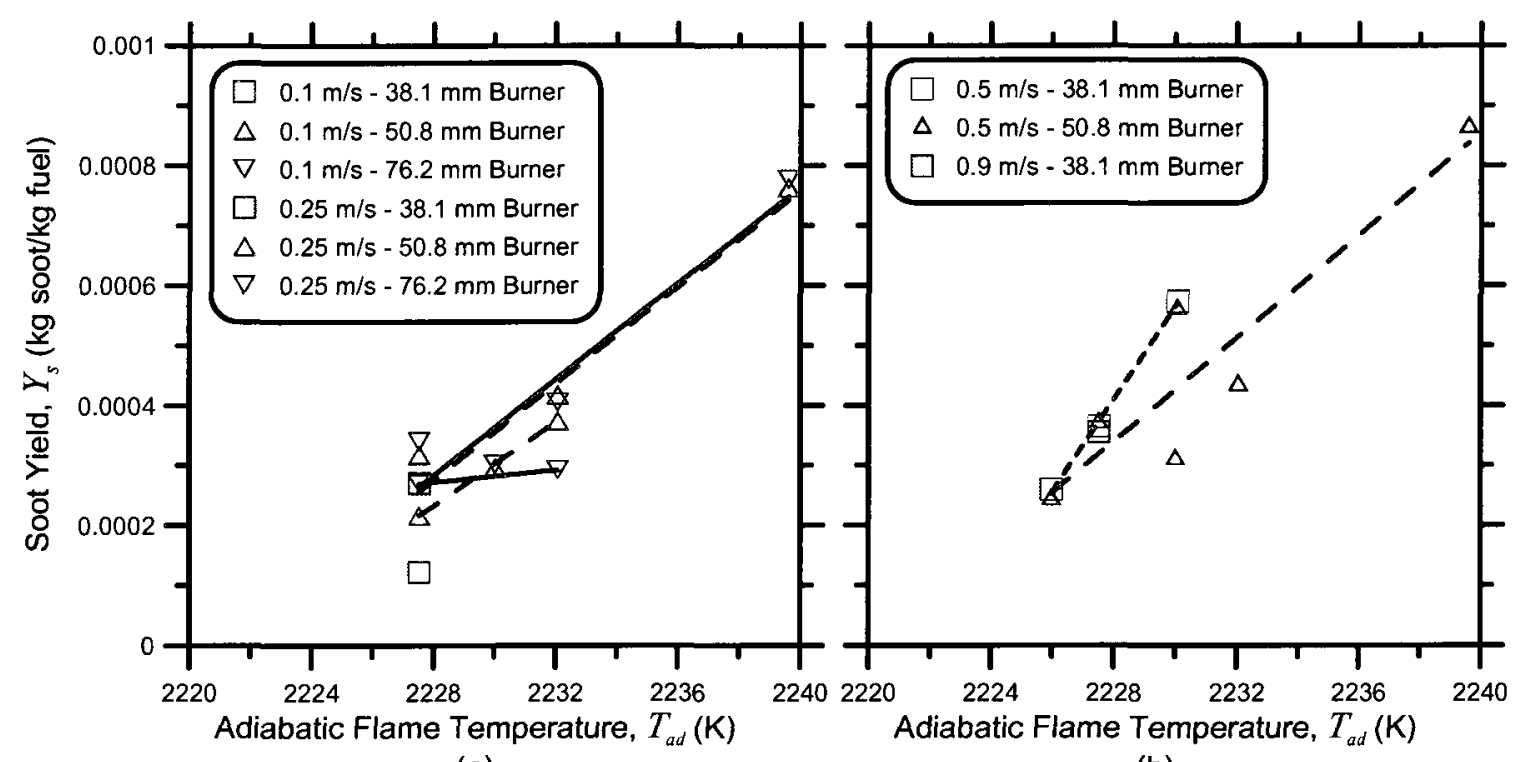

(a)

(b)

Figure 5.20 - Soot yield as a function of the adiabatic flame temperature determined using the NASA computer program CEA for values with a $F_{f}$ of (a) less than 0.003 and (b) greater than 0.003 . Lines represent linear fits of data

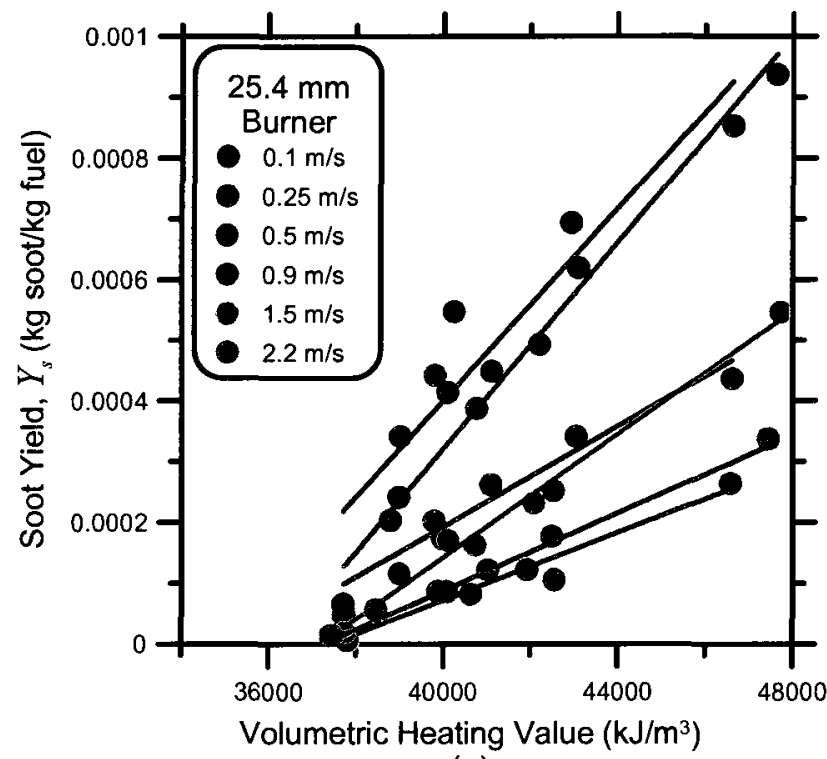

(a)

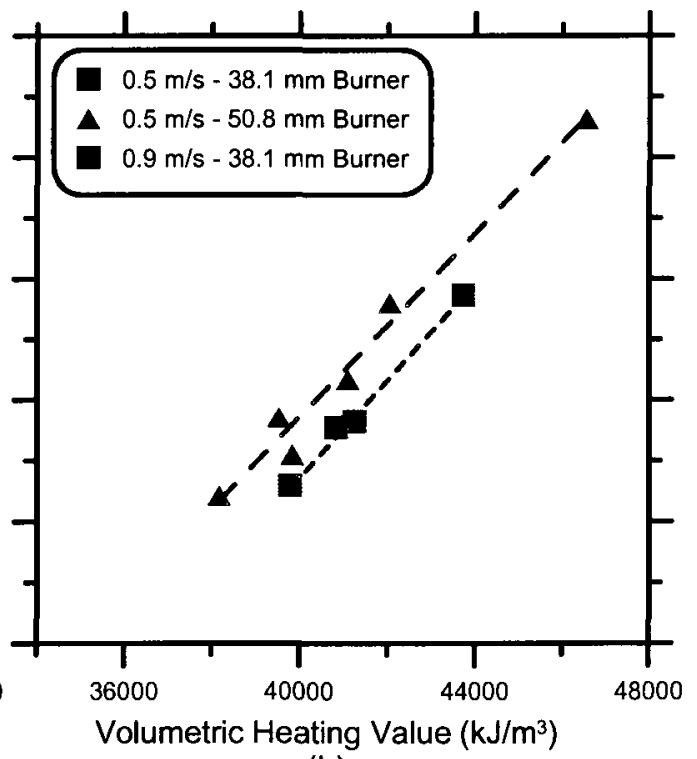

(b)

Figure 5.21 - Soot yield as a function of the volumetric heating value for (a) all $25.4 \mathrm{~mm}$ burner and (b) values with a $F_{f}$ of greater than 0.003 . Lines represent linear fits of data

Figure 5.21 (a) shows that for the range of fuel mixtures considered at any fixed

flow condition, i.e. fixed burner diameter and exit velocity, a linear relationship exists 
between the soot yield and the heating value. For $F r_{f}<0.003$ (shown by the $25.4 \mathrm{~mm}$ burner data of Figure 5.21 (a)), the slope varies with different exit velocities, indicating that the relative importance of fuel chemistry (heating value) changes at different flow conditions. For $F r_{f}>0.003$ (Figure 5.21(b)), which is the regime where soot yield was hypothesized to be constant with flow condition, there is a strong correlation of soot yield with the volumetric heating value, notwithstanding the limited range of currently available data with which to test this correlation.

\subsection{Implications}

As mentioned at the outset of this thesis, by ignoring crosswind effects which typically reduce soot yield, a worst-case scenario approach has been considered. If we continue to apply the worst-case scenario approach, we can neglect the effects of soot yield at fire Froude numbers less than 0.003 , since the soot yield decreases at fire Froude numbers less than 0.003 for all burner nozzle diameters tested. Thus, the soot yield appears to be a function of the heating value as shown in Figure 5.22 for the narrow range of data considered. This range includes burners of $38.1 \mathrm{~mm}, 50.8 \mathrm{~mm}$, and $76.2 \mathrm{~mm}$ in diameter. The fuel range includes all of the six fuel mixtures, and includes heating values between $\sim 38,000 \mathrm{~kJ} / \mathrm{m}^{3}$ and $\sim 47,000 \mathrm{~kJ} / \mathrm{m}^{3}$. The flow range includes fire Froude numbers greater than 0.003 , and more specifically, exit velocities greater than $0.5 \mathrm{~m} / \mathrm{s}$. The correlation is surprisingly good, and the scatter of the points in Figure 5.22 is well within the uncertainty shown by the error bars, which indicate uncertainties of less than $21 \%$. While it must be stressed that there is not enough data to conclude that this trend will properly estimate soot emissions from flares of stack diameter up to $101.6 \mathrm{~mm}$, the data are promising. Also of concern is how the soot yield will vary at fire Froude numbers larger 
than the tested range. Thus, the reader is cautioned that because of the empirical nature of the correlation, it should be regarded as applicable only over the limited range of conditions tested. However, this new model is still a significant improvement over the single emission factor approach currently adopted, that has been shown to be based on data of questionable relevance.

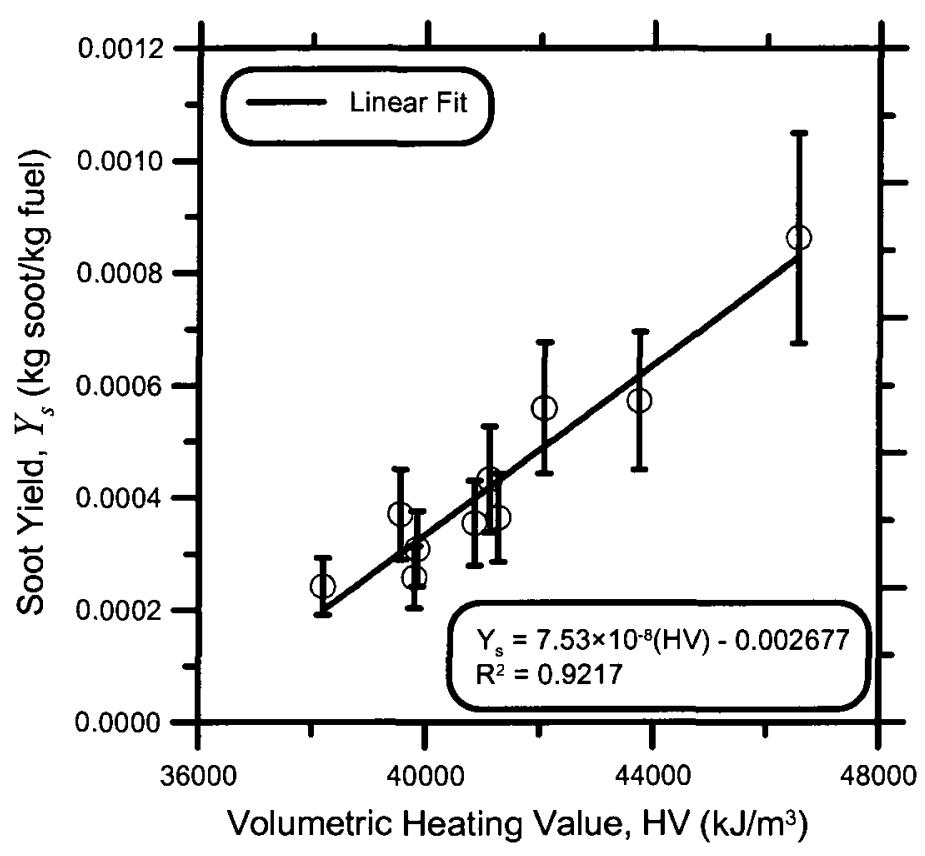

Figure 5.22 - Soot yield as a function of heating value for burners with diameters larger than $38.1 \mathrm{~mm}$ and fire Froude numbers greater than 0.003

Figure 5.23 is a reproduction of Figure 5.22 with the vertical axis re-scaled in terms of a volume-based emission factor $(E F)$, corresponding to the soot emission rate factor used in the CAPP guide [2007]. The linear relationship for the present limited data set would predict an $E F$ of approximately $0.58 \mathrm{~kg} \mathrm{soot} / 10^{3} \mathrm{~m}^{3}$ of fuel for a fuel with a heating value of $45,000 \mathrm{~kJ} / \mathrm{m}^{3}$, much less than $2.5632 \mathrm{~kg} \mathrm{soot} / 10^{3} \mathrm{~m}^{3}$ of fuel value currently suggested by CAPP [2007]. The difference in these values could represent a significant difference for estimates of soot produced from flaring solution gas. Despite the limitations of the present data and given the origins of the current CAPP emission 
factor, the present model may be a more representative emission factor to describe emissions from solution gas flares. However, the range of conditions and fuels used must be expanded before the present relationship can be applied with confidence in regular industry practice.

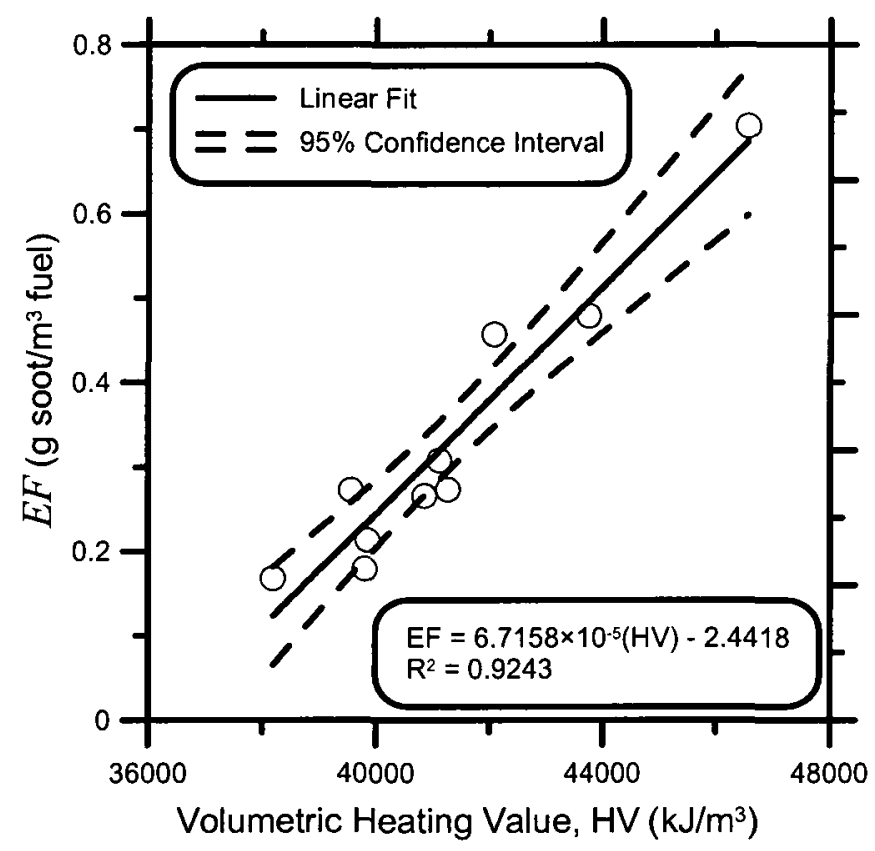

Figure 5.23 - $E F$ as a function of heating value for burners with diameters larger than $38.1 \mathrm{~mm}$ and fire Froude numbers greater than $\mathbf{0 . 0 0 3}$ 


\section{Chapter 6}

\section{Conclusions and Recommendations}

\subsection{Conclusions}

Soot emissions from lab-scale flares have been measured for several fuel mixtures representative of solution gas in Alberta. A new sampling protocol was developed based on the recommendations of Canteenwalla [2007] to account for errors related to the dilution ratio of the dilution tunnel. The new sampling protocol was developed through a consolidation of recommendations from several different standards originally intended for diesel engines and stationary exhaust stacks. The new protocol was implemented with two measurement techniques: Laser-Induced Incandescence (LII) and gravimetric sampling. The gravimetric sampling system not only provided a basis to confirm the LII-generated results, but also yielded a unique method for comparing the ratio of elemental carbon (EC) to organic carbon (OC) present in the measured soot.

From the experiments, it was found that the soot yield (defined as the ratio of mass of soot produced to mass of fuel burned) was no greater than $0.001 \mathrm{~kg}$ soot $/ \mathrm{kg}$ fuel for the heaviest sooting fuel mixture, and the soot yield changed depending on the flow conditions. The results were compared with the limited amount of published literature 
available, however the flame regime and fuel compositions studied in the present work had not been considered by previous researchers. The fire Froude number was a parameter suggested in the literature for scaling turbulent diffusion flames, and it was shown that for burners where the diameter was larger than $38.1 \mathrm{~mm}$, the soot yield appeared to approach an asymptotic value as the fire Froude number increased beyond approximately 0.003 . The scaling of flow effects below this fire Froude number could not be solved by any of the simple scaling laws applied in this thesis. However, for the data above a fire Froude number of 0.003 where flow effects appeared to be minimized, the soot yield scaled linearly with fuel heating value over the targeted range of fuel mixtures relevant to typical solution gas compositions directed to flares.

Soot emission factors were compared to data from available sources of the USEPA [2009] WebFIRE database as well as values currently used by the upstream oil and gas industry in Canada supplied by CAPP. Analysis of existing emission factors revealed a three order of magnitude range in soot yield values, which appear in part to be due to reporting errors in the WebFIRE database. Results from the currently available data suggest the emission factor for a solution gas flare mixture with a heating value of $45 \mathrm{MJ} / \mathrm{m}^{3}$ would be approximately $0.58 \mathrm{~kg}$ soot $/ 10^{3} \mathrm{~m}^{3}$ as opposed to the 2.5632 value currently suggested in the CAPP [2007] NPRI reporting guide. For a very rough order of magnitude estimate, considering gas flared volumes of 135 billion $\mathrm{m}^{3} /$ year as estimated from satellite data [Elvidge et al., 2009], and estimating a single valued soot emission factor of $0.58 \mathrm{~kg}$ soot $/ 10^{3} \mathrm{~m}^{3}$, flaring could produce approximately $78.3 \mathrm{Gg}$ of soot annually. While this is low compared to the global estimate of $4400 \mathrm{Gg}$ of soot annually, considering the concentration of flaring in the North within Canada and other parts of the 
world (namely Russia), the potential magnitude of soot emissions from flares is quite important as the deposition of these particles on snow is a serious concern in terms of surface albedo alteration.

It is important to note that the current work is not suggesting a single emission factor for estimating soot, but providing a preliminary empirical relationship between the fuel heating value and emission factors. If fuel composition is known at a particular flare site, an emission factor based on the fuel could be used. This would be an important improvement over the current single emission factor approach, especially considering the questionable origins of the factors currently available.

\subsection{Recommendations}

It is recommended that further work be undertaken to expand the current data set to include larger flow rates and larger burner diameters in particular. A larger range of fire Froude numbers would provide critical additional information to aid in the understanding of soot yield behaviour at fire Froude numbers beyond the range of the current data for burner diameters larger than $38.1 \mathrm{~mm}$. A direct measurement of the residence time could be made using a Laser Doppler Velocimetry (LDV) system. Measurements of the ratio of elemental carbon to organic carbon present in soot performed using standard test procedures could further establish that the ratio of an LII to a gravimetric measurement is indeed indicative of the $\mathrm{EC}$ to $\mathrm{OC}$ ratio.

In terms of the experimental setup, a new burner could be designed which allows for direct control on the burner temperature by means of a water-cooling system to directly measure any effects of fuel pre-heating on the soot yield. Different turbulence generating grids could be used to investigate the effects of cold flow turbulence on the 
soot emission. As the facility currently exists, there is not an a great deal of opportunity to increase the flow rate beyond what was done in the current work, and a new enclosure and dilution tunnel system would most likely be needed to increase the fuel flow rates significantly. To investigate the effects of crosswinds on soot yield, the LSF would need to be operated in a wind tunnel with the ability to collect and measure particulates. 


\section{References}

Acrivos, A., \& Taylor, T. D. (1962). Heat and Mass Transfer from Single Spheres in Stokes Flow. The Physics of Fluids, 5(4), 387-394.

AirLiquide. (2008). MSDS sheet for Hydrogen Sulfide. Retrieved October, 2009 from http://www.airliquide.ca/file/otherelement/pj/hydrogen_sulfide_air_liquide_canada _english(us)49525.pdf.

ANSI/ASME. (1985). ANSI/ASME PTC 19.1 - Part 1 - Measurement Uncertainty, Instruments and Apparatus.

Appel, J., Bockhorn, H., \& Frenklach, M. (2000). Kinetic modeling of soot formation with detailed chemistry and physics: laminar premixed flames of $\mathrm{C} 2$ hydrocarbons. Combustion and Flame, 121(1-2), 122-136.

ARB. (2008). Stationary Source Test Methods Volume 6-Quality Assurance in the Testing of Stationary Sources. ARB. Retrieved March, 2009 from http://www.arb.ca.gov/testmeth/vol6/vol6.htm.

BCOGC. (2008). Flaring, Incineration and Venting. Well Testing. Retrieved January, 2010 from www.ogc.gov.bc.ca.

Becker, H. A., \& Liang, D. (1978). Visible Length of Vertical Free Turbulent Diffusion Flames. Combustion and Flame, 32, 115-137.

Becker, H. A., \& Liang, D. (1982). Total emission of soot and thermal radiation by free turbulent diffusion flames. Combustion and Flame, 44, 305-318.

Becker, H. A., \& Yamazaki, S. (1978). Entrainment, Momentum Flux and Temperature in Vertical Free Turbulent Diffusion Flames. Combustion and Flame, 33, 123-149.

Blake, T. R., \& McDonald, M. (1993). An Examination of Flame Length Data from Vertical Turbulent Diffusion Flames. Combustion and Flame, 94, 426-432. 
Bockhorn, H., Geitlinger, H., Jungfleisch, B., Lehre, T., Schön, A., Streibel, T., \& Stuntz, R. (2002). Progress in characterization of soot formation by optical methods. Physical Chemistry Chemical Physics, 4(15), 3780-3793.

Bond, T. C., Wehner, B., Plewka, A., Wiedensohler, A., Heintzenberg, J., \& Charlson, R. J. (2006). Climate-relevant properties of primary particulate emissions from oil and natural gas combustion. Atmospheric Environment, 40(19), 3574-3587.

Bond, T. C., Bhardwaj, E., Dong, R., Jogani, R., Jung, S., Roden, C., Streets, D. G., \& Trautmann, N. M. (2007). Historical emissions of black and organic carbon aerosol from energy-related combustion, 1850-2000. Global Biogeochemical Cycles, 21(2), 1-16.

Brock, T. D. (1983). Membrane Filtration: A User's Guide and Reference Manual (pp. 310-337). Madison, WI: Science Tech Publishers.

Calcote, H. F., \& Manos, D. M. (1983). Effect of molecular structure on incipient soot formation. Combustion and Flame, 49(1-3), 289-304.

Canteenwalla, P. M. (2007). Soot emissions from turbulent diffusion flames burning simple alkane fuels. M.A.sc Thesis. Carleton University.

CAPP. (2007). A Recommended Approach to Completing the National Pollutant Release Inventory (NPRI) for the Upstream Oil and Gas Industry. Retrieved September, 2008 from http://www.capp.ca/library/publications/policyRegulatory/pages/pubInfo .aspx?DocId=119572\#a3C7EUiuvHbf.

Chang, M. C., Chow, J. C., Watson, J. G., Hopke, P. K., Yi, S. M., England, G. C. (2004). Measurement of Ultrafine Particle Size Distributions from Coal-, Oil-, and Gas-Fired Stationary Combustion Sources. Journal of the Air \& Waste Management Association, 54, 1494-1505.

Choi, M. Y., Mulholland, G. W., Hamins, A., \& Kashiwagi, T. (1995). Comparisons of the Soot Volume Fraction Using Gravimetric and Light Extinction Techniques. Combustion and Flame, 102, 161-169.

Delichatsios, M. A. (1993a). Transition from Momentum to Buoyancy-Controlled Turbulent Jet Diffusion Flames and Flame Height Relationships. Combustion and Flame, 92, 349-364.

Delichatsios, M. A. (1993b). Smoke yields from turbulent buoyant jet flames. Fire Safety Journal, 20(4), 299-311.

Dobbins, R. A., Mulholland, G. W., \& Bryner, N. P. (1994). Comparison of a fractal smoke optics model with light extinction measurements. Atmospheric Environment, 28(5), 889-897. 
EC. (2005). Flaring in the Petroleum Industry. Environment Canada. Retrieved January 2010 from http://ec.gc.ca/cleanairairpur/caol/OGEB/oilgas/flaring/flaring_general2_e.htm.

ERCB. (2009). ST60B-2009 - Upstream Petroleum Industry Flaring and Venting Report: Industry Performance for Year Ending December 31, 2008. Calgary, AB.

Eklund, B., Anderson, E. P., Walker, B. L., \& Burrows, D. B. (1998). Characterization of Landfill Gas Composition at the Fresh Kills Municipal Solid-Waste Landfill. Environmental Science \& Technology, 32, 2233-2237.

Ellzey, J. L., Berbee, J. G., Tay, Z. F., \& Foster, D. E. (1990). Total soot yield from a propane diffusion flame in cross-flow. Combustion Science and Technology, 71, 41-52.

Elvidge, C. D., Ziskin, D., Baugh, K. E., Tuttle, B. T., Ghosh, T., Pack, D. W., Erwin, E. H., \& Zhizhin, M. (2009). A Fifteen Year Record of Global Natural Gas Flaring Derived from Satellite Data. Energies, 2, 595-622.

Frenklach, M., \& Warnatz, J. (1987). Detailed Modeling of PAH Profiles in a Sooting Low-Pressure Acetylene Flame. Combustion Science and Technology, 51, 265-283.

Glassman, I. (1998). Sooting laminar diffusion flames: Effect of dilution, additives, pressure, and microgravity. Proceedings of the Combustion Institute, 27(1), 15891596.

Goldstein, R. J. (1996) Physical laws of fluid mechanics and their application to measurement techniques. Experimental Methods in Fluid Mechanics (2 ed.). Washington, DC: Taylor and Francis.

Guo, H., Liu, F., Smallwood, G. J., \& Gülder, Ö. L. (2002). The flame preheating effect on numerical modelling of soot formation in a two-dimensional laminar ethyleneair diffusion flame. Combustion Theory and Modelling, 6(2), 173-187.

Hansen, J., Sato, M., Ruedy, R., Lacis, A., \& Oinas, V. (2000). Global warming in the twenty-first century: An alternative scenario. Proceedings of the National Academy of Sciences of the United States of America, 97(18), 9875-9880.

Holman, J. P. (2002). Heat Transfer (9 ed., pp. 607-608). New York: McGraw-Hill.

Hottel, H. C., \& Hawthorne, W. R. (1949). Symposium on Combustion and Flame and Explosion Phenomena, 3, 254.

IPCC. (2007). Climate Change 2007: The Physical Science Basis. Contribution of Working Group I to the Fourth Assessment Report of the Intergovernmental Panel on Climate Change. In S. Solomon, D. Qin, M. Manning, Z. Chen, M. Marquis, K. 
Averyt, M. Tignor, \& H. Miller. (p. 996). Cambridge, UK and New York, USA: Cambridge University Press.

John, W., \& Reischl, G. (1978). Measurements of the filtration efficiencies of selected filter types. Atmospheric Environment, 12, 2015-2019.

Johnson, M. R., \& Kostiuk, L. W. (2000). Efficiencies of low-momentum jet diffusion flames in crosswinds. Combustion and Flame, 123(1-2), 189-200.

Johnson, M. R., Spangelo, J. L., \& Kostiuk, L. W. (2001a). A Characterization of Solution Gas Flaring in Alberta. Journal of the Air \& Waste Management Association, 51, 1167-1177.

Johnson, M. R., Wilson, D. J., \& Kostiuk, L. W. (2001b). A fuel stripping mechanism for wake-stabilized jet diffusion flames in crossflow. Combustion Science and Technology, 169, 155-174.

Kent, J. H. (1986). A Quantitative Relationship Between Soot Yield and Smoke-point Measurements. Combustion and Flame, 63, 349-358.

Kent, J. H., \& Wagner, H. G. (1984). Temperature and Fuel Effects in Sooting Diffusion Flames. Proceedings of the Combustion Institute, 20, 1007-1015.

Kostiuk, L. W., Johnson, M. R., \& Thomas, G. (2004). University of Alberta Flare Research Project Final Report. Edmonton. Retrieved July, 2009 from http://www.mece.ualberta.ca/groups/combustion/flare/papers/Final\%20Report2004. pdf

Kostiuk, L. W., Majeski, A. J., Poudenx, P., Johnson, M. R., \& Wilson, D. J. (2000). Scaling of wake-stabilized jet diffusion flames in a transverse air stream. Proceedings of the Combustion Institute, 28(1), 553-559.

Köylü, Ü. Ö., \& Faeth, G. M. (1992). Structure of overfire soot in buoyant turbulent diffusion flames at long residence times. Combustion and Flame, 89(2), 140-156.

McDaniel, M. (1983). EPA-600/2-83-052 - Flare Efficiency Study. Austin, TX: USEPA.

McKinnon, J. T., \& Howard, J. B. (1992). The roles of PAH and acetylene in soot nucleation and growth. Proceedings of the Combustion Institute, 24, 963-972.

NASA. (2004). Chemical Equilibrium with Applications (CEA). Glenn Research Center.

Neoh, K. G., Howard, J. B., \& Sarofin, A. F. (1982). Particulate carbon, formation during combustion (pp. 261-282). Plenum Press. 
Olson, D. B., Pickens, J. C., \& Gill, R. J. (1985). The Effects of Molecular Structure on Soot Formation II. Diffusion Flames. Combustion and Flame, 62, 43-60.

Ouf, F., Vendel, J., Coppalle, A., Weill, M., \& Yon, J. (2008). Characterization of Soot Particles in the Plumes of Over-Ventilated Diffusion Flames. Combustion Science and Technology, 180(4), 674-698.

Peters, N., \& Göttgens, J. (1991). Scaling of Buoyant Turbulent Jet Diffusion Flames. Combustion and Flame, 85, 206-214.

Pohl, J. H., Lee, J., Payne, R., \& Tichenor, B. A. (1986). Combustion efficiency of flares. Combustion science and technology, 50(4-6), 217-231. Taylor and Francis.

Pope III, C. A., Burnett, R. T., Thun, M. J., Calle, E. E., Krewski, D., Ito, K., \& Thurston, G.D. (2002). Lung Cancer, Cardiopulmonary Mortality, and Long-term Exposure to Fine Particulate Air Pollution. The Journal of the American Medical Association, 287(9), 1132-1141.

Prybysh, R. (2002). The Production of Toxic Emissions from Reacting Diffusion Jet Flames in a Crossflow. M.A.sc. Thesis. University of Alberta.

Ramanathan, V., \& Carmichael, G. (2008). Global and regional climate changes due to black carbon. Nature Geoscience, 1(4), 221-227.

Richter, H., \& Howard, J. B. (2000). Formation of polycyclic aromatic hydrocarbons and their growth to soot - a review of chemical reaction pathways. Progress in Energy and Combustion Science, 26, 565-608.

Santoro, R. J., \& Semerjian, H. G. (1984). Soot Formation in Diffusion Flames: Flow Rate, Fuel Species and Temperature Effects. Proceedings of the Combustion Institute, 20, 997-1006.

Schalla, R. L., \& McDonald, G. E. (1953). Variation in Smoking Tendency Among Hydrocarbons of Low Molecular Weight. Industrial and Engineering Chemistry, 45(7), 1497-1500.

Senkan, S., \& Castaldi, M. (1996). Formation of polycyclic aromatic hydrocarbons $(\mathrm{PAH})$ in methane combustion: Comparative new results from premixed flames. Combustion and Flame, 107, 141-150.

Sivathanu, Y. R., \& Faeth, G. M. (1990). Soot volume fractions in the overfire region of turbulent diffusion flames. Combustion and Flame, 81(2), 133-149.

Sivathanu, Y. R., Gore, J. P., \& Faeth, G. M. (1988). Scalar properties in the overfire region of sooting turbulent diffusion flames. Combustion and Flame, 73(3), $315-$ 329. 
Skjøth-Rasmussen, M. S., Glarbor, P., Ostberg, M., Johannessen, J. T., Livbjerg, H., Jensen, A. D., \& Christensen, T. S. (2004). Formation of polycyclic aromatic hydrocarbons and soot in fuel-rich oxidation of methane in a laminar flow reactor. Combustion and Flame, 136, 91-128.

Smedley, J. M., Williams, A., \& Bartle, K. D. (1992). A mechanism for the formation of soot particles and soot deposits. Combustion and Flame, 91, 71-82.

Smith, C. R., Tracy, G. W., \& Farrar, R. L. (1992). Applied Reservoir Engineering, Vol. 1 (pp. 3-2 - 3-3). Tulsa, OK: Oil \& Gas Consultants International.

Smooke, M. D., Mcenally, C. S., Pfefferle, L. D., Hall, R. J., \& Colket, M. B. (1999). Computational and experimental study of soot formation in a coflow, laminar diffusion flame. Combustion and Flame, 117(1-2), 117-139.

Snelling, D. R., Smallwood, G. J., Liu, F., Gülder, Ö. L., \& Bachalo, W. D. (2005). A calibration independent laser-induced incandescence technique for soot measurement by detecting absolute light intensity. Applied Optics, 44(31), 67736785 .

Strosher, M. T. (2000). Characterization of emissions from diffusion flare systems. Journal of the Air \& Waste Management Association, 50(10), 1723-33.

Takagi, T., Shin, H., \& Ishio, A. (1980). Local laminarization in turbulent diffusion flames. Combustion and Flame, 37, 163-170.

Taylor, E. (2006). Filter Weighing Procedure for 2007 and Newer Heavy Duty Diesel Engines. M.Sc. Thesis. West Virginia University.

Trottier, S., Guo, H., Smallwood, G. J., \& Johnson, M. R. (2007). Measurement and modeling of the sooting propensity of binary fuel mixtures. Proceedings of the Combustion Institute, 31, 611-619.

Tsai, C., Chang, C., Shih, B., Aggarwal, S. G., Li, S., Chein, H. M., \& Shih, T. (2002). The effect of environmental conditions and electrical charge on the weighing accuracy of different filter materials. The Science of the Total Environment, 293, 201-206.

Turns, S. R. (2000). An Introduction to Combustion: Concepts and Applications (2 ed.). USA: McGraw-Hill.

UNECE. (2008). Regulation No. 49 - Uniform Provisions Concerning the Measures to be Taken Against the Emissions of Gaseous and Particulate Pollutants from Compression-Ignition Engines for Use in Vehicles, and the Emission of Gaseous Pollutants from Positive-Ignition Eng. 
USEIA. (2008). International Energy Statistics: Petroleum Production. Retrieved December, 2009 from http://tonto.eia.doe.gov/cfapps/ipdbproject/iedindex3.cfm?tid $=3 \&$ pid $=43 \&$ aid $=1 \&$ cid $=\&$ syid $=2004 \&$ eyid $=2008 \&$ unit $=$ BCF.

USEPA. (1991). Data from flaring landfill gas, Confidential Report.

USEPA. (1994). TP 714C - Diesel particulate filter handling and weighing.

USEPA. (1995). AP-42 Section 13.5 - Miscellaneous Sources: Industrial Flares.

USEPA. (2000). 40 CFR Part 60 Test Method 5 - Determination of Particulate Matter Emissions from Stationary Sources. Retrieved January, 2009 from http://www.epa.gov/ttn/emc/promgate.html.

USEPA. (2005). 40 CFR Part 1065 Engine-Testing Procedures. Federal Register. Retrieved January, 2009 from http://ecfr.gpoaccess.gov/cgi/t/text/text$\mathrm{idx}$ ?c=ecfr\&tpl=/ecfrbrowse/Title40/40cfr1065_main_02.tpl.

USEPA. (2008). EPA/600/R-08/139 - Integrated Science Assessment for Particulate Matter (External Draft Review). Washington, DC. Retrieved January, 2009 from http://edocket.access.gpo.gov/2008/E8-30197.htm.

USEPA. (2009). WebFIRE - Technology Transfer Network, Clearinghouse for Inventories and Emission Factors. Retrieved April, 2009 from http://cfpub.epa.gov/oarweb/index.cfm?action=fire.main.

Wu, J. S., Krishnan, S. S., \& Faeth, G. M. (1997). Refractive indices at visible wavelengths of soot emitted from buoyant turbulent diffusion flames. Journal of Heat Transfer, 119(2), 230-238. 


\section{Appendix A}

\section{Conversion of Soot Emission Factors in AP-42 to NPRI units}

The original factors as reported in the USEPA AP-42 [1991] Section 13.5 document are: $0 \mu \mathrm{g} / \mathrm{L}$ for non-sooting; $40 \mu \mathrm{g} / \mathrm{L}$ for light sooting; $177 \mu \mathrm{g} / \mathrm{L}$ for average sooting; $274 \mu \mathrm{g} / \mathrm{L}$ for heavy sooting. The fuel used during these tests was $100 \%$ propylene as determined by a review of the McDaniel [1983] report, even though US EPA AP-42 suggested it was a mixture composed of $80 \%$ propylene and $20 \%$ propane (See Chapter 1, page 12, Table 1.1). Calculations are shown for the "average sooting" case.

First convert to standard units:

$177 \frac{\mu \mathrm{g}}{\mathrm{L}}=177 \times 10^{-6} \frac{\mathrm{kg}}{\mathrm{m}_{\text {exhaust }}^{3}}$

Unfortunately, the dilution of the sample was not measured as stated in McDaniel [1983]. However, it is still possible to estimate a magnitude of the emission factor by assuming that the sample was drawn from a point well within the plume and dilution effects might be negligible. Any dilution in the actual samples would thus result in a somewhat higher soot yield than that estimated in this calculation. 
Assuming complete combustion and negligible dilution at the sampling point, a relation between $Q_{\text {exhaust }}$ and $Q_{\text {fuel }}$ can be calculated as follows:

$$
\begin{aligned}
& C_{\alpha} H_{\beta}+\left(\alpha+\frac{\beta}{4}\right)\left(O_{2}+3.76 N_{2}\right) \Rightarrow \alpha C O_{2}+\frac{\beta}{2} H_{2} O+3.76\left(\alpha+\frac{\beta}{4}\right) N_{2} \\
& \therefore \frac{Q_{\text {exhaust }}}{Q_{\text {fiel }}}=\alpha+\beta / 2+3.76\left(\alpha+\frac{\beta}{4}\right)=4.76 \alpha+1.44 \beta
\end{aligned}
$$

For $100 \%$ propylene:

$$
\begin{aligned}
& C_{\alpha} H_{\beta}=C_{3} H_{6} \\
& \therefore \alpha=3, \beta=6 \\
& \therefore \frac{Q_{\text {exhaust }}}{Q_{\text {fiel }}}=22.92 \frac{\mathrm{m}_{\text {exhaust }}^{3}}{\mathrm{~m}_{\text {fuel }}^{3}}
\end{aligned}
$$

Based on the reported fuel composition, a new PM emission factor, $E F$ can then be estimated as:

$$
E F=177 \times 10^{-6} \frac{\mathrm{kg}}{\mathrm{m}_{\text {exhaust }}^{3}} \times 22.92 \frac{\mathrm{m}_{\text {exhaust }}^{3}}{\mathrm{~m}_{\text {fuel }}^{3}}=4.0568 \times 10^{-3} \frac{\mathrm{kg}}{\mathrm{m}_{\text {fitel }}^{3}}
$$

Note that as stated above this estimate should represent a lower bound to the actual measured emission factor, since it would be practically impossible to avoid some entrainment of air (and hence some dilution of the plume) at the sample point. In the absence of other available information, we have necessarily assumed an undiluted sample.

The table below summarizes the recalculated emission factor data. 
Table A.1 - Estimated, corrected Emissions Factors based on source data from McDaniel [1983]

\begin{tabular}{|c|c|c|c|c|}
\hline $\begin{array}{c}\text { Original } \\
\text { Factor } \\
(\mathbf{\mu g} / \mathbf{L})\end{array}$ & $\begin{array}{c}\text { Original } \\
\text { Factor } \\
\left(\mathbf{k g} / \mathbf{m}^{\mathbf{3}}\right)\end{array}$ & $\begin{array}{c}\text { Converted to } \\
\text { per volume } \\
\text { fuel }\left(\mathbf{k g} / \mathbf{m}^{\mathbf{3}}\right)\end{array}$ & $\begin{array}{c}\text { Converted to } \\
\mathbf{N P R I ~ u n i t s} \\
\left(\mathbf{k g} / \mathbf{1 0}^{\mathbf{3}} \mathbf{~ m}^{\mathbf{3}}\right)\end{array}$ & $\begin{array}{c}\text { Converted based on HHV (85.5 to 45 } \\
\mathbf{M J} / \mathbf{m}^{\mathbf{3}} \text { assuming emitted PM scales } \\
\text { linearly with heating value) }\left(\mathbf{k g} / \mathbf{1 0}^{\mathbf{3}} \mathbf{~ m}^{\mathbf{3}}\right)\end{array}$ \\
\hline 40 & 0.00004 & 0.0009168 & 0.9168 & 0.4825 \\
\hline 177 & 0.000177 & 0.0040568 & 4.0568 & 2.1352 \\
\hline 274 & 0.000274 & 0.0062801 & 6.2801 & 3.3053 \\
\hline $\begin{array}{c}\text { CAPP } \\
\text { Factor } \\
{[\mathbf{2 0 0 7 ]}}\end{array}$ & & & 0.85 for landfill \\
gas $(\mathrm{HHV}=\sim 15)$ & \\
\hline
\end{tabular}




\section{Appendix B}

\section{Mass Flow Controller Calibrations and Methods}

As recommended by the manufacturer, the mass flow controllers (MFC) used in this research were recalibrated every year after purchase. The methane and butane controllers were purchased new and used within the first year of purchase, so retained their factory calibration values. For the other MFCs one of two methods was used to calibrate the controllers depending on the full-scale range. For flows of up to 30 LPM a bubble flow meter (Model - Sensidyne Gilibrator-2) was used. For flow rates larger than 30 LPM, either the Gilibrator was used or calibration was achieved by directly measuring the mass of gas displaced through the MFC from an 80 -size pressurized gas bottle and pressure regulator placed on a scale (Model - A\&D GP-40K). The methods are described in detail below.

\section{Gilibrator MFC Calibration}

A Gilibrator operates by calculating the amount of time required for a bubble to pass between two infrared sensors in a vertical tube. The volume between the two sensors is known and the time step is measured, allowing for calculation of the volume flow rate. 
Calibration points were taken at MFC set point voltages of $0.25,0.5,0.75,1.0$, $1.75,2.5,3.75,4.25,4.75$, and 4.95 volts ( 5 volts is the maximum set point voltage). For the propane calibration, an extra calibration point was taken at a set point voltage of 0.1 volts. Measurements were recorded for the barometric pressure, ambient temperature, measured MFC voltage, and Gilibrator flow rate. Ten tests were completed at each set point voltage, and sample averages were calculated for each of the measured values. The Gilibrator flow rates were given in units of $\mathrm{L} / \mathrm{min}\left(Q_{L P M}\right)$ and were converted to standard units $\left(Q_{S L P M}\right)$ based on the measured ambient barometric pressure and temperature.

The average standard flow rate was plotted as a function of the average measured MFC voltage for each set point. The linear regression of these data produced a calibration relation of standard flow rate as a function of measured MFC voltage. The values for slopes and intercepts for all calibrations are listed in Table B.1. The linear fits were considered appropriate, as the coefficient of determination $\left(\mathrm{R}^{2}\right)$ was greater than or equal to 0.9999 .

The uncertainty was calculated based on the ANSI/ASME [1985] measurement uncertainty method as presented in Chapter 3. The systematic error was calculated based on the Gilibrator instrument error ( $\pm 1 \%$ of reading), the barometric pressure uncertainty $( \pm 0.06646 \mathrm{~Pa})$, and the temperature measurement uncertainty $( \pm 2.2 \mathrm{~K})$. The precision error was calculated based on the 10 individual calculations of $Q_{S L P M}$ and was combined with the systematic error as described in Chapter 3 to produce the total uncertainty, displayed in Table B.1. Of specific note is that the uncertainty calculated by this method produced a percent uncertainty as a function of the measurement, as opposed to a 
function of the full scale. This allowed for a reduced uncertainty while operating in the low flow regimes of the flow controller (i.e. $<10 \%$ Full-scale (F.S.)).

\section{Mass-displacement MFC Calibration}

For flow rates larger than 30 LPM, or if the Gilibrator was not available (which was the case for the Ethane calibration), a scale connected to a computerized data acquisition system was used to measure the mass displacement of gas from a pressurized gas bottle through the MFC per unit time as a means of determining the mass flow rate. A small gas bottle (size 80 or Q) of the gas intended for use was placed on the scale (along with the MFC, pressure regulator, and required tubing) and the change in mass with time was measured to determine the mass flow rate.

Calibration points were taken at MFC set point voltages of $0.25,0.5,0.75,1.0$, $1.75,2.5,3.25,4.0,4.5$, and 4.95 volts. The scale was connected to a computer via a RS-232 output and the mass of the gas bottle was recorded using a LabVIEW program. Once the flow meter had been set and the gas was flowing, data logging was initiated and the drop in mass was measured over time to calculate the mass flow rate. The test was terminated once $50 \mathrm{~g}$ of gas had passed through the MFC. The standard flow rate was calculated based on the mass flow rate recorded and the standard density of the gas according to the ideal gas law.

The individual flow rates were plotted as a function of the measured MFC voltage in the same manner previously described to produce a linear relationship between the standard flow rate and the measured MFC voltage. The slope and intercept determined from the linear fits are shown in Table B.1. 
The uncertainty was calculated for this calibration based on the instrument uncertainty of the scale. Specifications for the scale provide a linearity error of $\pm 1 \mathrm{~g}$ and a repeatability error of $0.5 \mathrm{~g}$. These elemental errors were combined to give an instrument uncertainty of $\pm 1.118 \mathrm{~g}$. Since the mass flow rate was a mass difference divided by the test time, two measurements of mass were made, each contributing \pm $1.118 \mathrm{~g}$ of uncertainty. The ANSI/ASME [1985] uncertainty method produced a final standard volume flow rate uncertainty of approximately $\pm 3.15 \%$ of reading. It should be noted that if a mass of $150 \mathrm{~g}$ were passed through the flow meter, the uncertainty would be less than $1 \%$ of reading, but was not done in the current calibrations. All MFC uncertainties are displayed in Table B.1.

Table B.1 - Calibration values for MFCs

\begin{tabular}{|c|c|c|c|c|c|c|c|}
\hline $\begin{array}{c}\text { Brooks } \\
\text { MFC Model }\end{array}$ & Fuel & $\begin{array}{c}\text { Range } \\
\text { (SLPM) }\end{array}$ & $\begin{array}{c}\text { Slope } \\
\text { (SLPM/Volt) }\end{array}$ & $\begin{array}{l}\text { Intercept } \\
\text { (SLPM) }\end{array}$ & $\mathbf{R}^{2}$ & Uncertainty & $\begin{array}{c}\text { Calibration } \\
\text { Method }\end{array}$ \\
\hline $5853 \mathrm{~S} / \mathrm{BE}$ & Methane & $0-100$ & 1 & 0 & - & $\pm 1 \%$ F.S. & Factory \\
\hline $5851 \mathrm{~S} / \mathrm{BD}$ & Ethane & $0-20$ & 3.8582 & -0.1775 & 1 & $\begin{array}{c} \pm 3.15 \% \text { of } \\
\text { Reading }\end{array}$ & Scale \\
\hline $5850 \mathrm{~S} / \mathrm{BC}$ & Propane & $0-5.1$ & 1.0408 & -0.0098 & 0.9999 & $\begin{array}{c} \pm 1.25 \% \text { of } \\
\text { Reading }\end{array}$ & Gilibrator \\
\hline $5850 \mathrm{~S} / \mathrm{BA}$ & Butane & $0-3$ & 1 & 0 & - & $\pm 1 \%$ F.S. & Factory \\
\hline $5850 \mathrm{~S} / \mathrm{BC}$ & $\mathrm{CO}_{2}$ & $0-3.5$ & 0.6915 & 0.0218 & 0.9999 & $\begin{array}{c} \pm 1.25 \% \text { of } \\
\text { Reading }\end{array}$ & Gilibrator \\
\hline $5850 \mathrm{~S} / \mathrm{BC}$ & Nitrogen & $0-2.6$ & 0.5239 & 0.0084 & 1 & $\begin{array}{c} \pm 1.22 \% \text { of } \\
\text { Reading }\end{array}$ & Gilibrator \\
\hline
\end{tabular}




\section{Appendix C}

\section{Measurement of Fuel Gas Temperature}

As mentioned previously in Section 5.2, temperature measurements were made slightly upstream of the burner exit plane to measure the fuel gas temperature. The burner tube is heated and hence the fuel is heated via heat transfer from the flame. It was suggested in the literature that an elevated burner temperature could lead to higher soot emissions [Smooke et al., 1999; Guo et al., 2002]. This effect was shown to be negligible in the current work for the range of flow conditions considered. The method by which the fuel temperature was measured is detailed here nonetheless.

A 1/8" O.D. K-type thermocouple manufactured by Omega was used to measure the gas stream temperature. The orientation of the probe is shown schematically in Figure C.1. For the smallest burner $(12.7 \mathrm{~mm}$ I.D.), the thermocouple blocked approximately $6.25 \%$ of the burner area, which would increase the exit velocity by approximately $6.67 \%$. The increase in exit velocity drops to $1.59 \%$ for the $25.4 \mathrm{~mm}$ I.D. burner. To ensure that the slight increase in exit velocity or the thermocouple probe itself did not affect the soot yield, soot yield measurements were made with, and without the 
thermocouple probe in the flow, and there was no measureable difference in soot yield values.

The thermocouple was bent into an "L" shape so that the tip of the thermocouple probe would be facing upstream in the burner pipe, reducing the amount of radiation to the thermocouple probe tip from the flame. The probe tip was inserted into the burner so that the tip was positioned approximately $25 \mathrm{~mm}$ upstream of the exit plane and centered inside each burner. The horizontal section of the thermocouple lead was placed slightly above the exit plane so that it did not contact the burner tip as shown in Figure C.1.

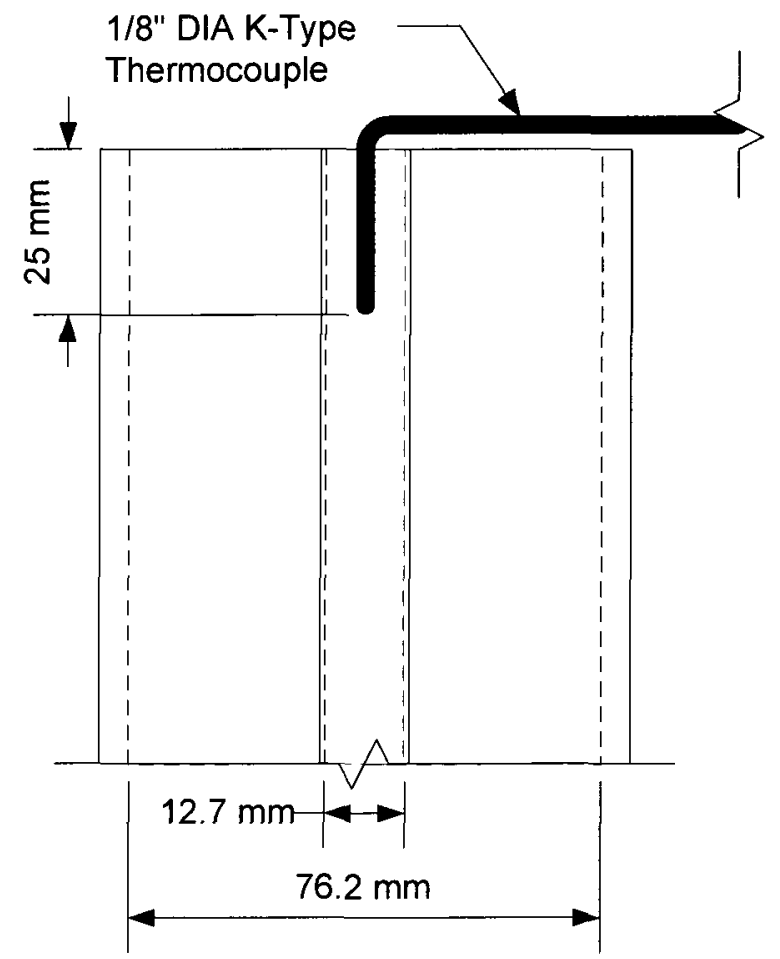

Figure C.1 - Schematic of temperature measurement setup showing both the $12.7 \mathrm{~mm}$ and $76.2 \mathrm{~mm}$ diameter burners

The temperature measured by the thermocouple was determined by the overall energy balance on the junction. Under the assumption that the burner inside wall temperature was greater than the temperature of the thermocouple junction, which was in 
turn greater than the actual fuel temperature, the general form of the energy balance was given by Equation C.1:

$$
h A\left(T_{j}-T_{\text {fitel }}\right)=k A \varepsilon F\left(T_{w}^{4}-T_{j}^{4}\right)
$$

where $h$ is the heat transfer coefficient, $A$ is the surface area of the thermocouple junction, $T_{j}$ is the junction temperature, $T_{f u e l}$ is the actual fuel gas temperature, $T_{w}$ is the temperature of the inner burner walls, $k$ is the Stefan-Boltzman constant, $\varepsilon$ is the emissivity of the junction, and $F$ is the view factor between the junction and the burner walls. Direct radiation to the thermocouple junction from the flame was assumed negligible due to the orientation, which minimized the flame view factor, and conduction along the thermocouple leads was neglected. Equation C.1 was rearranged and simplified to solve for the actual fuel gas temperature, as shown in Equation C.2.

$$
T_{\text {fiel }}=T_{j}-\frac{k \varepsilon F}{h}\left(T_{w}^{4}-T_{j}^{4}\right)
$$

For the shape factor, $F$, a value of 1.0 was used since the entire junction was exposed to the inner walls of the burner. For the junction, an emissivity value of 0.98 for oxidized Nickel-Chromium wire was used [Holman, 2002], which corresponded to the $\mathrm{Ni}$-Chromium and $\mathrm{Ni}$-Aluminum K-type thermocouple junction. A value of $62^{\circ} \mathrm{C}$ was taken from Canteenwalla [2007] for the burner inner wall temperature $25 \mathrm{~mm}$ below the exit plane. The heat transfer coefficient was calculated using the empirical correlation for the Nusselt number suggested by Acrivos and Taylor [1962] (Equation C.3) for heat transfer from spheres as cited in McEnally et al. [1997], which is used for an approximation of the heat transfer coefficient at the thermocouple junction from the Nusselt number definition (Equation C.5). Gas stream properties were calculated at the junction temperature for methane to determine the heat transfer coefficient. 


$$
N u=2+\frac{1}{2} P e+\frac{1}{4} P e^{2} \ln P e+0.03404 P e^{2}+\frac{1}{16} P e^{3} \ln P e
$$

where

$$
\begin{gathered}
P e=R e \cdot P r \\
N u=\frac{h d}{k_{f}}
\end{gathered}
$$

For an average junction temperature of $45.1^{\circ} \mathrm{C}$ measured by the thermocouple, the true temperature would be $43.6^{\circ} \mathrm{C}$ for the minimum and maximum exit velocities. For all cases, the agreement between the measured and true temperatures was within $1 \%$, which shows that the correction is negligible for these cases. The method shown here has been checked against the theory of Becker and Yamazaki [1978], which yields identical results when used with the current properties. 


\section{Appendix D}

\section{Experimental Test Conditions}

All test conditions are shown in the following tables. Soot yield results included in the tables were derived from the LII-based measurements.

Table D.1 - Average 6-component fuel mixture test conditions $(M W=19.19 \mathrm{~kg} / \mathrm{kmol}$; $\mu=1.087 \times 10^{-5}$ Pa-s; $\left.\rho=0.789 \mathrm{~kg} / \mathrm{m}^{3} ; \mathrm{HHV}=40,400 \mathrm{~kJ} / \mathrm{m}^{3}\right)$

\begin{tabular}{ccccccc}
\hline $\begin{array}{c}\text { Burner } \\
\text { I.D. } \\
(\mathbf{m m})\end{array}$ & $\begin{array}{c}\text { Fuel Mass } \\
\text { Flow Rate } \\
(\mathbf{k g} / \mathbf{s})\end{array}$ & $\begin{array}{c}\text { Burner Exit } \\
\text { Velocity } \\
(\mathbf{m} / \mathbf{s})\end{array}$ & $\begin{array}{c}\text { Reynolds } \\
\text { Number }\end{array}$ & $\begin{array}{c}\text { Fire } \\
\text { Froude } \\
\text { Number }\end{array}$ & $\begin{array}{c}\text { Soot Yield } \\
\text { (kg soot/kg fuel) }\end{array}$ & $\begin{array}{c}\text { Soot Yield } \\
\text { Uncertainty } \\
(\mathbf{k g} \text { soot/kg fuel) }\end{array}$ \\
\hline 12.7 & $5.32 \mathrm{E}-05$ & 0.58 & 532.8 & $8.50 \mathrm{E}-03$ & $8.74 \mathrm{E}-05$ & $2.81 \mathrm{E}-05$ \\
12.7 & $9.60 \mathrm{E}-05$ & 1.03 & 962.1 & $1.53 \mathrm{E}-02$ & $4.95 \mathrm{E}-05$ & $1.24 \mathrm{E}-05$ \\
12.7 & $1.61 \mathrm{E}-04$ & 1.69 & 1610.3 & $2.54 \mathrm{E}-02$ & $5.99 \mathrm{E}-05$ & $1.35 \mathrm{E}-05$ \\
12.7 & $1.72 \mathrm{E}-04$ & 1.79 & 1720.2 & $2.75 \mathrm{E}-02$ & $8.87 \mathrm{E}-05$ & $1.99 \mathrm{E}-05$ \\
12.7 & $3.87 \mathrm{E}-04$ & 4.01 & 3879.4 & $6.12 \mathrm{E}-02$ & $8.69 \mathrm{E}-05$ & $1.98 \mathrm{E}-05$ \\
12.7 & $5.38 \mathrm{E}-04$ & 5.56 & 5395.0 & $8.54 \mathrm{E}-02$ & $8.28 \mathrm{E}-05$ & $1.75 \mathrm{E}-05$ \\
12.7 & $8.07 \mathrm{E}-04$ & 8.40 & 8098.3 & $1.29 \mathrm{E}-01$ & $6.52 \mathrm{E}-05$ & $1.42 \mathrm{E}-05$ \\
25.4 & $4.23 \mathrm{E}-05$ & 0.12 & 212.1 & $1.19 \mathrm{E}-03$ & $8.52 \mathrm{E}-05$ & $3.29 \mathrm{E}-05$ \\
25.4 & $9.96 \mathrm{E}-05$ & 0.27 & 499.2 & $3.03 \mathrm{E}-03$ & $1.73 \mathrm{E}-04$ & $4.67 \mathrm{E}-05$ \\
25.4 & $2.14 \mathrm{E}-04$ & 0.56 & 1075.1 & $6.34 \mathrm{E}-03$ & $5.47 \mathrm{E}-04$ & $1.17 \mathrm{E}-04$ \\
25.4 & $3.87 \mathrm{E}-04$ & 1.02 & 1941.5 & $1.15 \mathrm{E}-02$ & $4.13 \mathrm{E}-04$ & $8.85 \mathrm{E}-05$ \\
25.4 & $6.46 \mathrm{E}-04$ & 1.70 & 3238.9 & $1.85 \mathrm{E}-02$ & $1.70 \mathrm{E}-04$ & $3.54 \mathrm{E}-05$ \\
25.4 & $9.48 \mathrm{E}-04$ & 2.50 & 4754.1 & $2.74 \mathrm{E}-02$ & $8.61 \mathrm{E}-05$ & $1.83 \mathrm{E}-05$ \\
38.1 & $9.61 \mathrm{E}-05$ & 0.12 & 321.3 & $9.69 \mathrm{E}-04$ & $1.22 \mathrm{E}-04$ & $3.07 \mathrm{E}-05$ \\
38.1 & $2.41 \mathrm{E}-04$ & 0.29 & 806.5 & $2.42 \mathrm{E}-03$ & $2.70 \mathrm{E}-04$ & $6.01 \mathrm{E}-05$ \\
38.1 & $4.84 \mathrm{E}-04$ & 0.57 & 1618.0 & $4.84 \mathrm{E}-03$ & $3.65 \mathrm{E}-04$ & $7.82 \mathrm{E}-05$ \\
38.1 & $8.72 \mathrm{E}-04$ & 1.03 & 2917.0 & $8.87 \mathrm{E}-03$ & $3.55 \mathrm{E}-04$ & $7.55 \mathrm{E}-05$ \\
50.8 & $1.71 \mathrm{E}-04$ & 0.12 & 429.9 & $8.70 \mathrm{E}-04$ & $2.15 \mathrm{E}-04$ & $4.99 \mathrm{E}-05$ \\
50.8 & $4.30 \mathrm{E}-04$ & 0.29 & 1078.3 & $2.19 \mathrm{E}-03$ & $3.18 \mathrm{E}-04$ & $6.87 \mathrm{E}-05$ \\
50.8 & $8.62 \mathrm{E}-04$ & 0.58 & 2161.3 & $4.39 \mathrm{E}-03$ & $3.71 \mathrm{E}-04$ & $7.96 \mathrm{E}-05$ \\
76.2 & $3.87 \mathrm{E}-04$ & 0.12 & 647.2 & $7.07 \mathrm{E}-04$ & $2.69 \mathrm{E}-04$ & $5.91 \mathrm{E}-05$ \\
76.2 & $9.69 \mathrm{E}-04$ & 0.30 & 1620.5 & $1.79 \mathrm{E}-03$ & $3.40 \mathrm{E}-04$ & $7.16 \mathrm{E}-05$ \\
\hline & & & & & & \\
\hline
\end{tabular}


Table D.2 - Light 6-component fuel mixture test conditions ( $M W=17.94 \mathrm{~kg} / \mathrm{kmol}$; $\left.\mu=1.094 \times 10^{-5}-\mathrm{Pa}-\mathrm{s} ; \rho=0.738 \mathrm{~kg} / \mathrm{m}^{3} ; \mathrm{HHV}=38,900 \mathrm{~kJ} / \mathrm{m}^{3}\right)$

\begin{tabular}{ccccccc}
\hline $\begin{array}{c}\text { Burner } \\
\text { I.D. } \\
\text { (mm) }\end{array}$ & $\begin{array}{c}\text { Fuel Mass } \\
\text { Flow Rate } \\
(\mathbf{k g} / \mathbf{s})\end{array}$ & $\begin{array}{c}\text { Burner Exit } \\
\text { Velocity } \\
\text { (m/s) }\end{array}$ & $\begin{array}{c}\text { Reynolds } \\
\text { Number }\end{array}$ & $\begin{array}{c}\text { Froude } \\
\text { Number }\end{array}$ & $\begin{array}{c}\text { Soot Yield } \\
\text { (kg soot/kg fuel) }\end{array}$ & $\begin{array}{c}\text { Soot Yield } \\
\text { Uncertainty } \\
\text { ( kg soot/kg fuel) }\end{array}$ \\
\hline 25.4 & $9.98 \mathrm{E}-05$ & 0.30 & 495.0 & $2.98 \mathrm{E}-03$ & $2.03 \mathrm{E}-04$ & $4.93 \mathrm{E}-05$ \\
25.4 & $2.00 \mathrm{E}-04$ & 0.57 & 993.9 & $5.98 \mathrm{E}-03$ & $3.41 \mathrm{E}-04$ & $7.33 \mathrm{E}-05$ \\
25.4 & $3.62 \mathrm{E}-04$ & 1.02 & 1797.4 & $1.08 \mathrm{E}-02$ & $2.41 \mathrm{E}-04$ & $5.59 \mathrm{E}-05$ \\
25.4 & $6.05 \mathrm{E}-04$ & 1.67 & 2999.3 & $1.84 \mathrm{E}-02$ & $1.16 \mathrm{E}-04$ & $2.48 \mathrm{E}-05$ \\
25.4 & $8.88 \mathrm{E}-04$ & 2.47 & 4406.0 & $2.76 \mathrm{E}-02$ & $5.62 \mathrm{E}-05$ & $1.19 \mathrm{E}-05$ \\
38.1 & $4.53 \mathrm{E}-04$ & 0.58 & 1499.3 & $4.84 \mathrm{E}-03$ & $2.59 \mathrm{E}-04$ & $5.56 \mathrm{E}-05$ \\
50.8 & $8.07 \mathrm{E}-04$ & 0.58 & 2002.0 & $4.39 \mathrm{E}-03$ & $2.42 \mathrm{E}-04$ & $5.13 \mathrm{E}-05$ \\
\hline
\end{tabular}

Table D.3 - Heavy 6-component fuel mixture test conditions $(M W=21.48 \mathrm{~kg} / \mathrm{kmol}$; $\left.\mu=1.072 \times 10^{-5} \mathrm{~Pa}-\mathrm{s} ; \rho=0.883 \mathrm{~kg} / \mathrm{m}^{3} ; \mathrm{HHV}=42,900 \mathrm{~kJ} / \mathrm{m}^{3}\right)$

\begin{tabular}{ccccccc}
\hline $\begin{array}{c}\text { Burner } \\
\text { I.D. } \\
(\mathbf{m m})\end{array}$ & $\begin{array}{c}\text { Fuel Mass } \\
\text { Flow Rate } \\
(\mathbf{k g} / \mathbf{s})\end{array}$ & $\begin{array}{c}\text { Burner Exit } \\
\text { Velocity } \\
(\mathbf{m} / \mathbf{s})\end{array}$ & $\begin{array}{c}\text { Reynolds } \\
\text { Number }\end{array}$ & $\begin{array}{c}\text { Fire } \\
\text { Froude } \\
\text { Number }\end{array}$ & $\begin{array}{c}\text { Soot Yield } \\
\text { (kg soot/kg fuel) }\end{array}$ & $\begin{array}{c}\text { Soot Yield } \\
\text { Uncertainty } \\
( \pm \mathbf{~ k g ~ s o o t / k g ~ f u e l ) ~}\end{array}$ \\
\hline 12.7 & $5.92 \mathrm{E}-05$ & 0.58 & 605.1 & $8.63 \mathrm{E}-03$ & $2.31 \mathrm{E}-04$ & $6.94 \mathrm{E}-05$ \\
25.4 & $4.74 \mathrm{E}-05$ & 0.12 & 242.5 & $1.21 \mathrm{E}-03$ & $1.05 \mathrm{E}-04$ & $3.83 \mathrm{E}-05$ \\
25.4 & $1.19 \mathrm{E}-04$ & 0.30 & 609.2 & $3.02 \mathrm{E}-03$ & $3.41 \mathrm{E}-04$ & $8.10 \mathrm{E}-05$ \\
25.4 & $2.39 \mathrm{E}-04$ & 0.57 & 1222.7 & $6.12 \mathrm{E}-03$ & $6.94 \mathrm{E}-04$ & $1.50 \mathrm{E}-04$ \\
25.4 & $4.31 \mathrm{E}-04$ & 1.01 & 2204.3 & $1.10 \mathrm{E}-02$ & $6.19 \mathrm{E}-04$ & $1.42 \mathrm{E}-04$ \\
25.4 & $7.18 \mathrm{E}-04$ & 1.68 & 3667.7 & $1.90 \mathrm{E}-02$ & $2.52 \mathrm{E}-04$ & $5.71 \mathrm{E}-05$ \\
25.4 & $1.06 \mathrm{E}-03$ & 2.46 & 5402.0 & $2.80 \mathrm{E}-02$ & $1.77 \mathrm{E}-04$ & $3.73 \mathrm{E}-05$ \\
38.1 & $5.40 \mathrm{E}-04$ & 0.57 & 1839.1 & $4.96 \mathrm{E}-03$ & $5.73 \mathrm{E}-04$ & $1.23 \mathrm{E}-04$ \\
50.8 & $9.60 \mathrm{E}-04$ & 0.59 & 2454.3 & $4.47 \mathrm{E}-03$ & $5.60 \mathrm{E}-04$ & $1.17 \mathrm{E}-04$ \\
\hline
\end{tabular}

Table D.4 - Average 4-component fuel mixture test conditions $(M W=18.60 \mathrm{~kg} / \mathrm{kmol}$; $\mu=1.065 \times 10^{-5} \mathrm{~Pa}-\mathrm{s} ; \rho=0.765 \mathrm{~kg} / \mathrm{m}^{3} ; \mathrm{HHV}=41,400 \mathrm{~kJ} / \mathrm{m}^{3}$ )

\begin{tabular}{ccccccc}
\hline $\begin{array}{c}\text { Burner } \\
\text { I.D. } \\
(\mathbf{m m})\end{array}$ & $\begin{array}{c}\text { Fuel Mass } \\
\text { Flow Rate } \\
(\mathbf{k g} / \mathbf{s})\end{array}$ & $\begin{array}{c}\text { Burner Exit } \\
\text { Velocity } \\
(\mathbf{m} / \mathbf{s})\end{array}$ & $\begin{array}{c}\text { Reynolds } \\
\text { Number }\end{array}$ & $\begin{array}{c}\text { Froude } \\
\text { Number }\end{array}$ & $\begin{array}{c}\text { Soot Yield } \\
\text { (kg soot/kg fuel) }\end{array}$ & $\begin{array}{c}\text { Soot Yield } \\
\text { Uncertainty } \\
( \pm \mathbf{~ k g ~ s o o t / k g ~ f u e l ) ~}\end{array}$ \\
\hline 25.4 & $4.08 \mathrm{E}-05$ & 0.12 & 209.4 & $1.02 \mathrm{E}-03$ & $1.21 \mathrm{E}-04$ & $4.62 \mathrm{E}-05$ \\
25.4 & $9.58 \mathrm{E}-05$ & 0.27 & 491.0 & $2.39 \mathrm{E}-03$ & $2.61 \mathrm{E}-04$ & $6.47 \mathrm{E}-05$ \\
25.4 & $2.07 \mathrm{E}-04$ & 0.57 & 1062.2 & $5.18 \mathrm{E}-03$ & $4.48 \mathrm{E}-04$ & $9.78 \mathrm{E}-05$ \\
25.4 & $3.74 \mathrm{E}-04$ & 1.01 & 1918.0 & $9.09 \mathrm{E}-03$ & $4.92 \mathrm{E}-04$ & $1.09 \mathrm{E}-04$ \\
25.4 & $6.25 \mathrm{E}-04$ & 1.69 & 3201.2 & $1.53 \mathrm{E}-02$ & $2.32 \mathrm{E}-04$ & $4.84 \mathrm{E}-05$ \\
25.4 & $9.17 \mathrm{E}-04$ & 2.49 & 4698.1 & $2.25 \mathrm{E}-02$ & $1.22 \mathrm{E}-04$ & $2.59 \mathrm{E}-05$ \\
50.8 & $1.65 \mathrm{E}-04$ & 0.12 & 423.9 & $7.28 \mathrm{E}-04$ & $3.74 \mathrm{E}-04$ & $8.79 \mathrm{E}-05$ \\
50.8 & $4.16 \mathrm{E}-04$ & 0.29 & 1066.2 & $1.82 \mathrm{E}-03$ & $4.18 \mathrm{E}-04$ & $8.88 \mathrm{E}-05$ \\
50.8 & $8.34 \mathrm{E}-04$ & 0.58 & 2135.7 & $3.69 \mathrm{E}-03$ & $4.33 \mathrm{E}-04$ & $9.45 \mathrm{E}-05$ \\
76.2 & $3.74 \mathrm{E}-04$ & 0.13 & 639.3 & $5.98 \mathrm{E}-04$ & $2.92 \mathrm{E}-04$ & $6.27 \mathrm{E}-05$ \\
76.2 & $9.38 \mathrm{E}-04$ & 0.30 & 1601.5 & $1.51 \mathrm{E}-03$ & $4.06 \mathrm{E}-04$ & $8.55 \mathrm{E}-05$ \\
\hline
\end{tabular}


Table D.5 - Light 4-component fuel mixture test conditions $(M W=17.93 \mathrm{~kg} / \mathrm{kmol} ; \mu=$ $1.075 \times 10^{-5} \mathrm{~Pa}-\mathrm{s} ; \rho=0.737 \mathrm{~kg} / \mathrm{m}^{3} ; \mathrm{HHV}=40,100 \mathrm{~kJ} / \mathrm{m}^{3}$ )

\begin{tabular}{ccccccc}
\hline $\begin{array}{c}\text { Burner } \\
\text { I.D. } \\
(\mathbf{m m})\end{array}$ & $\begin{array}{c}\text { Fuel Mass } \\
\text { Flow Rate } \\
(\mathbf{k g} / \mathbf{s})\end{array}$ & $\begin{array}{c}\text { Burner Exit } \\
\text { Velocity } \\
(\mathbf{m} / \mathbf{s})\end{array}$ & $\begin{array}{c}\text { Reynolds } \\
\text { Number }\end{array}$ & $\begin{array}{c}\text { Froude } \\
\text { Number }\end{array}$ & $\begin{array}{c}\text { Soot Yield } \\
\text { (kg soot/kg fuel) }\end{array}$ & $\begin{array}{c}\text { Soot Yield } \\
\text { Uncertainty } \\
\text { (土 kg soot/kg fuel) }\end{array}$ \\
\hline 25.4 & $9.26 \mathrm{E}-05$ & 0.28 & 468.8 & $2.49 \mathrm{E}-03$ & $2.01 \mathrm{E}-04$ & $4.91 \mathrm{E}-05$ \\
25.4 & $2.00 \mathrm{E}-04$ & 0.57 & 1011.4 & $5.37 \mathrm{E}-03$ & $4.42 \mathrm{E}-04$ & $9.54 \mathrm{E}-05$ \\
25.4 & $3.61 \mathrm{E}-04$ & 1.01 & 1824.5 & $9.48 \mathrm{E}-03$ & $3.87 \mathrm{E}-04$ & $8.24 \mathrm{E}-05$ \\
25.4 & $6.03 \mathrm{E}-04$ & 1.69 & 3053.5 & $1.59 \mathrm{E}-02$ & $1.62 \mathrm{E}-04$ & $3.34 \mathrm{E}-05$ \\
25.4 & $8.86 \mathrm{E}-04$ & 2.48 & 4482.0 & $2.34 \mathrm{E}-02$ & $8.14 \mathrm{E}-05$ & $1.66 \mathrm{E}-05$ \\
50.8 & $4.02 \mathrm{E}-04$ & 0.31 & 1016.5 & $1.88 \mathrm{E}-03$ & $2.95 \mathrm{E}-04$ & $6.47 \mathrm{E}-05$ \\
50.8 & $8.05 \mathrm{E}-04$ & 0.58 & 2037.2 & $3.82 \mathrm{E}-03$ & $3.09 \mathrm{E}-04$ & $6.69 \mathrm{E}-05$ \\
76.2 & $9.06 \mathrm{E}-04$ & 0.30 & 1527.8 & $1.57 \mathrm{E}-03$ & $3.01 \mathrm{E}-04$ & $6.71 \mathrm{E}-05$ \\
\hline
\end{tabular}

Table D.6 - Heavy 4-component fuel mixture test conditions $(M W=21.46 \mathrm{~kg} / \mathrm{kmol}$; $\left.\mu=1.024 \times 10^{-5} \mathrm{~Pa}-\mathrm{s} ; \rho=0.883 \mathrm{~kg} / \mathrm{m}^{3} ; \mathrm{HHV}=46,900 \mathrm{~kJ} / \mathrm{m}^{3}\right)$

\begin{tabular}{ccccccc}
\hline $\begin{array}{c}\text { Burner } \\
\text { I.D. } \\
(\mathbf{m m})\end{array}$ & $\begin{array}{c}\text { Fuel Mass } \\
\text { Flow Rate } \\
(\mathbf{k g} / \mathbf{s})\end{array}$ & $\begin{array}{c}\text { Burner Exit } \\
\text { Velocity } \\
(\mathbf{m} / \mathbf{s})\end{array}$ & $\begin{array}{c}\text { Reynolds } \\
\text { Number }\end{array}$ & $\begin{array}{c}\text { Fire } \\
\text { Froude } \\
\text { Number }\end{array}$ & $\begin{array}{c}\text { Soot Yield } \\
\text { (kg soot/kg fuel) }\end{array}$ & $\begin{array}{c}\text { Soot Yield } \\
\text { Uncertainty } \\
( \pm \text { kg soot/kg fuel) }\end{array}$ \\
\hline 25.4 & $4.69 \mathrm{E}-05$ & 0.12 & 253.0 & $9.35 \mathrm{E}-04$ & $2.64 \mathrm{E}-04$ & $9.28 \mathrm{E}-05$ \\
25.4 & $1.10 \mathrm{E}-04$ & 0.27 & 590.7 & $2.19 \mathrm{E}-03$ & $4.36 \mathrm{E}-04$ & $1.07 \mathrm{E}-04$ \\
25.4 & $2.38 \mathrm{E}-04$ & 0.57 & 1279.4 & $4.74 \mathrm{E}-03$ & $8.53 \mathrm{E}-04$ & $1.85 \mathrm{E}-04$ \\
25.4 & $4.30 \mathrm{E}-04$ & 1.01 & 2308.4 & $8.38 \mathrm{E}-03$ & $9.37 \mathrm{E}-04$ & $2.05 \mathrm{E}-04$ \\
25.4 & $7.17 \mathrm{E}-04$ & 1.70 & 3849.9 & $1.39 \mathrm{E}-02$ & $5.46 \mathrm{E}-04$ & $1.15 \mathrm{E}-04$ \\
25.4 & $1.05 \mathrm{E}-03$ & 2.51 & 5652.0 & $2.07 \mathrm{E}-02$ & $3.37 \mathrm{E}-04$ & $7.05 \mathrm{E}-05$ \\
50.8 & $4.77 \mathrm{E}-04$ & 0.29 & 1282.4 & $1.68 \mathrm{E}-03$ & $7.63 \mathrm{E}-04$ & $1.67 \mathrm{E}-04$ \\
50.8 & $9.56 \mathrm{E}-04$ & 0.59 & 2568.0 & $3.37 \mathrm{E}-03$ & $8.63 \mathrm{E}-04$ & $1.87 \mathrm{E}-04$ \\
76.2 & $1.08 \mathrm{E}-03$ & 0.30 & 1927.0 & $1.38 \mathrm{E}-03$ & $7.77 \mathrm{E}-04$ & $1.69 \mathrm{E}-04$ \\
\hline
\end{tabular}

Table D.7 - Methane test conditions $\left(M W=16.043 \mathrm{~kg} / \mathrm{kmol} ; \mu=1.106 \times 10^{-5} \mathrm{~Pa}-\mathrm{s} ; \rho=0.66 \mathrm{~kg} / \mathrm{m}^{3}\right.$; $\mathrm{HHV}=37,700 \mathrm{~kJ} / \mathrm{m}^{3}$ )

\begin{tabular}{ccccccc}
\hline $\begin{array}{c}\text { Burner } \\
\text { I.D. } \\
(\mathbf{m m})\end{array}$ & $\begin{array}{c}\text { Fuel Mass } \\
\text { Flow Rate } \\
(\mathbf{k g} / \mathbf{s})\end{array}$ & $\begin{array}{c}\text { Burner Exit } \\
\text { Velocity } \\
(\mathbf{m} / \mathbf{s})\end{array}$ & $\begin{array}{c}\text { Reynolds } \\
\text { Number }\end{array}$ & $\begin{array}{c}\text { Fire } \\
\text { Froude } \\
\text { Number }\end{array}$ & $\begin{array}{c}\text { Soot Yield } \\
\text { (kg soot/kg fuel) }\end{array}$ & $\begin{array}{c}\text { Soot Yield } \\
\text { Uncertainty } \\
( \pm \text { kg soot/kg fuel) }\end{array}$ \\
\hline 25.4 & $3.48 \mathrm{E}-05$ & 0.12 & 170.0 & $1.17 \mathrm{E}-03$ & $5.38 \mathrm{E}-06$ & $2.64 \mathrm{E}-06$ \\
25.4 & $8.23 \mathrm{E}-05$ & 0.27 & 401.5 & $2.78 \mathrm{E}-03$ & $2.05 \mathrm{E}-05$ & $5.91 \mathrm{E}-06$ \\
25.4 & $1.79 \mathrm{E}-04$ & 0.56 & 874.0 & $6.05 \mathrm{E}-03$ & $6.58 \mathrm{E}-05$ & $1.38 \mathrm{E}-05$ \\
25.4 & $3.25 \mathrm{E}-04$ & 1.00 & 1584.1 & $1.10 \mathrm{E}-02$ & $4.79 \mathrm{E}-05$ & $1.38 \mathrm{E}-05$ \\
25.4 & $5.43 \mathrm{E}-04$ & 1.66 & 2648.9 & $1.83 \mathrm{E}-02$ & $1.90 \mathrm{E}-05$ & $3.95 \mathrm{E}-06$ \\
25.4 & $7.96 \mathrm{E}-04$ & 2.45 & 3887.3 & $2.72 \mathrm{E}-02$ & $1.39 \mathrm{E}-05$ & $2.98 \mathrm{E}-06$ \\
\hline
\end{tabular}

